University of Louisville

ThinkIR: The University of Louisville's Institutional Repository

8-2014

\title{
Numerical study of leading edge vortex circulation development on finite aspect ratio perching wings.
}

Kyle Hord

University of Louisville

Follow this and additional works at: https://ir.library.louisville.edu/etd

Part of the Mechanical Engineering Commons

\section{Recommended Citation}

Hord, Kyle, "Numerical study of leading edge vortex circulation development on finite aspect ratio perching wings." (2014). Electronic Theses and Dissertations. Paper 633.

https://doi.org/10.18297/etd/633

This Doctoral Dissertation is brought to you for free and open access by ThinkIR: The University of Louisville's Institutional Repository. It has been accepted for inclusion in Electronic Theses and Dissertations by an authorized administrator of ThinkIR: The University of Louisville's Institutional Repository. This title appears here courtesy of the author, who has retained all other copyrights. For more information, please contact thinkir@louisville.edu. 


\title{
NUMERICAL STUDY OF LEADING EDGE VORTEX CIRCULATION DEVELOPMENT ON FINITE ASPECT RATIO PERCHING WINGS
}

\author{
By \\ Kyle Hord \\ B.S, Mechanical Engineering 2009 \\ M.S., Mechanical Engineering 2010

\begin{abstract}
A Dissertation
Submitted to the Faculty of the

J.B. Speed School of Engineering University of Louisville in Partial Fulfillment of the Requirements

for the Degree of
\end{abstract}

Doctor of Philosophy

Department of Mechanical Engineering

University of Louisville

Louisville, Kentucky

August 2014 



\title{
NUMERICAL STUDY OF LEADING EDGE VORTEX CIRCULATION DEVELOPMENT ON FINITE ASPECT RATIO PERCHING WINGS
}

\author{
By \\ Kyle Hord \\ B.S, Mechanical Engineering 2009 \\ M.S., Mechanical Engineering 2010 \\ A Dissertation Approved On \\ July 31, 2014 \\ by the following Dissertation Committee: \\ Dr. Yongsheng Lian \\ Dissertation Director \\ Dr. Roger Bradshaw \\ Mechanical Engineering \\ Dr. Keith Sharp \\ Mechanical Engineering \\ Dr. Dar-Jen Chang \\ Computer Engineering and Computer Science \\ Dr. Andrew Wissink \\ U.S. Army Aeroflight Dynamics Directorate
}




\section{ACKNOWLEDGEMENTS}

My deepest gratitude goes to the following individuals for making this dissertation possible.

Yongsheng Lian for providing the opportunity to pursue my Ph.D even when there was no funding available. Finding support was a perpetual struggle, but he always was able to find it. He also had constant patience with me investing time into other projects unrelated to my research.

Ching-Ju Lai for supporting me with love and passion through the entirety of my studies. She encouraged me in times of doubt and frustration keeping me motivated to seeing my education through. She stayed patient with me during the times I was away for long periods for internship and study.

To my parents for their continuous and endless support, encouragement, and guidance throughout my education.

Finally to my friends, Daniel Porter, Russel Prater, Tim Broering, and Andrew Work for walking this path with me, and keeping my sanity in check through the years.

This work has been partially funded by the NASA Kentucky EPSCoR and the NASA Langley Research Summer Scholars program. 


\begin{abstract}
NUMERICAL STUDY OF LEADING EDGE VORTEX CIRCULATION DEVELOPMENT ON FINITE ASPECT RATIO PERCHING WINGS
\end{abstract}

Kyle Hord

July $31^{\text {st }}, 2014$

The perching maneuver of natural fliers is a complex motion involving fast change of angle of attack, complicated wing kinematics, large wing deformation and agile body motion control, but the prominent aerodynamic features can be revealed using a simple pitch-up wing motion coupled with a stream wise deceleration. In this dissertation the aerodynamic forces, the leading edge vorterx (LEV) development, and LEV circulation of pitch-up and perching wings are extensively studied at low Reynolds number conditions. 2D and 3D wings of different aspect ratios were linearly pitched up from $0^{\circ}$ to $90^{\circ}$ at three reduced pitch rates. The numerical investigation was conducted at a Reynolds number of 500 and the flow field was described using the unsteady three-dimensional incompressible Navier-Stokes equations on a set of composite overlapping grids. The Q-criterion was used to identify and isolate the LEV structure from shear layer vorticity. Results have shown that the LEV circulation depends primarily on the wing aspect ratio: increasing wing aspect ratio increased the rate of LEV circulation generation during the pitch-up motion. The reduced pitch rate for the pitch-up motion was found to delay the LEV circulation development when the aspect ratio was greater than two. For perching, pitch rate only altered the LEV propagation away from the wing, and not the magnitude of the circulation. 


\section{TABLE OF CONTENTS}

Page

ACKNOWLEDGEMENTS

ABSTRACT

iii

LIST OF TABLES

LIST OF FIGURES

ix

$\mathrm{x}$

\section{CHAPTER}

I INTRODUCTION 1

A. Lift Generation of Unsteady Wings . . . . . . . . . . . . . . 1

1. Perching Wings ............... 6

B. Objectives and Organization . . . . . . . . . . . . 7

II PERCHING MANEUVER KINEMATICS 10

A. Perching Simplification . . . . . . . . . . . . . . . 10

B. Pitching Motion .................. 11

C. Deceleration .................... 12

III NUMERICAL METHOD $\quad 15$

A. Navier-Stokes System of Equations . . . . . . . . . . . . . 15

B. Solving the Incompressible Navier Stokes Equations . . . . . . . 19

C. Boundary Conditions ................. 23

D. Rigid Body Motion . . . . . . . . . . . . . . . . . . 24

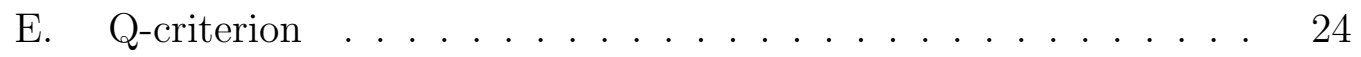

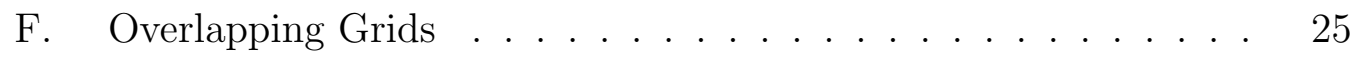

1. Types of Grid Generation . . . . . . . . . . . 25

G. Overlapping Structured Grids . . . . . . . . . . . . . . . . . . 30 
H. Overlapping Grid Assembly . . . . . . . . . . . . . . . . . 32

IV CODE VALIDATION 36

A. Shedding Cylinder .................. 36

B. 2D Pure Plunge . . . . . . . . . . . . . . . . 37

C. 2D Single Pitch . . . . . . . . . . . . . . . 39

$\begin{array}{lll}\text { V COMPUTATIONAL SETUP } & 41\end{array}$

A. Summary ..................... . . . 41

B. Grid Generation . . . . . . . . . . . . . . . 41

C. Domain Size . . . . . . . . . . . . . . . . . . . . . 44

D. Grid Sensitivity . . . . . . . . . . . . . . . 44

$\begin{array}{lll}\text { VI AERODYNAMIC ANALYSIS } & 48\end{array}$

A. Introduction . . . . . . . . . . . . . . . . . . 48

B. 2D Analysis ...................... . . 49

1. Effect of Reynolds Number . . . . . . . . . . . . . 49

2. 2D Pitch-up ................... 51

3. 2D Perching Wing ............... 53

C. 3D Analysis . . . . . . . . . . . . . . 56

1. Pitch-up and Perching Forces . . . . . . . . . 56

2. Aspect Ratio . . . . . . . . . . . . 58

3. Pitch Rate . . . . . . . . . . . . . 61

4. Perching ..................... 62

D. Spanwise Force Distribution . . . . . . . . . . . . . . . . 64

E. Pitching and Perching Conclusion . . . . . . . . . . . 76

VII VORTEX CIRCULATION AND LIFT 80

A. Introduction . . . . . . . . . . . . . . . 80

B. Noncirculatory Force Contribution . . . . . . . . . . . . 82 
C. Circulatory Lift Contribution . . . . . . . . . . . . 84

D. Circulatory and Noncirculatory Forces . . . . . . . . . 85

1. Noncirculatory Component . . . . . . . . . . . 86

2. Circulatory Component . . . . . . . . . . . . 87

E. 2D Vortex Size . . . . . . . . . . . . . . . . . . . . 89

F. Circulation and Lift Conclusion . . . . . . . . . . . . . . . 91

\section{GEOMETRY AND KINEMATIC EFFECTS ON CIRCULATION}

A. Introduction . . . . . . . . . . . . . . . . . 93

B. Mid and Quarter Span Circulation _............ 93

1. Pitching . . . . . . . . . . . . . . . 93

2. Perching ..................... 96

C. Spanwise LEV Circulation . . . . . . . . . . . . . . . . . . 98

1. Pitching . . . . . . . . . . . . . . . . 98

2. Perching . . . . . . . . . . . . . . 102

D. Circulation Conclusion . . . . . . . . . . . . . . . 105

IX FEATURE-BASED ADAPTATION 107

A. Summary . . . . . . . . . . . . . . . . . 107

B. Importance of Wake Grid Resolution . . . . . . . . . . . 107

C. Numerical Methods . . . . . . . . . . . . . . . . . . . . . 109

1. Temporal Discretization . . . . . . . . . . . 111

2. Feature-Based Adaptation . . . . . . . . . . . . 113

D. Richardson Extrapolation . . . . . . . . . . . . . 115

E. Grid Generation . . . . . . . . . . . . . . . . . 116

1. Flow Conditions and Kinematics . . . . . . . . . . . 118

F. Uniform Grid Refinement . . . . . . . . . . . . . . 120

1. $\quad 13 \pm 4^{o}$ Pitching Convergence . . . . . . . . . . . 121 
2. Convergence Error . . . . . . . . . . . . . 123

G. Feature-Based Adaptation . . . . . . . . . . . . . . . . 125

1. Adapted Mesh ................ . 127

2. Adapted Flow Field . . . . . . . . . . . . . . . 129

3. Adapted Forces and Error . . . . . . . . . . . 134

H. Conclusion ................. 136

$\begin{array}{llr}\text { X } & \text { CONCLUSIONS } & 138\end{array}$

A. Future Work . . . . . . . . . . . . . . . . . . 140

REFERENCES 142

$\begin{array}{ll}\text { APPENDIX } & 153\end{array}$

$\begin{array}{ll}\text { CURRICULUM VITAE } & 154\end{array}$ 


\section{LIST OF TABLES}

TABLE

Page

1 Comparison of CD with published results at varying Reynolds numbers 38

2 Three grids used in the grid sensitivity analysis . . . . . . . . . . 45

3 Effect of aspect ratio and $K$ on the pitch-up motion. . . . . . . . . 61

4 Coefficients for backwards difference schemes [1] . . . . . . . . . . . 112

5 Geometric settings for NACA0015 spatial study . . . . . . . . . . . 117

6 Flow conditions and kinematics used for pitching airfoil . . . . . . . . 119

$7 \quad$ Tested time steps $(\Delta t)$ used for temporal analysis, and the estimated

flow convection distance per time step $(\Delta x / c) \ldots \ldots . . \ldots . . . .119$ 


\section{LIST OF FIGURES}

FIGURE $\quad$ Page

1 Gliding ratio as a function of chord Reynolds number [2] . . . . . . . 2

2 Illustration of the key features in a dynamic stall phenomenon. (Left) Comparison of lift curves between static stall and dynamic stall; (Right) Evolution of dynamic stall vortex. (I) Start of the leading edge separation. (II) Vortex roll-up; (III) Stall onset and formation of a trailing edge vortex; (IV) Detachment of the dynamic stall vortex, and the sudden lift loss. . . . . . . . . . . . . . . . . . . 4

3 Angle from the horizontal plane at three different locations along a pigeon (Columba Livia) wing during three different flight modes. [3] . 10

4 Impact of the smoothing parameter and $K$ on the pitch-up kinematics. (Left) Change in angle of attack, (middle) angular velocity, and (right) acceleration of the wing. . . . . . . . . . . . . . 13

5 Declaration profile using smoothing function. (Left) Velocity (units per second) with respect to time (seconds). (Right) Declaration with respect to time. . . . . . . . . . . . . . . . . . . 14

$6 \quad$ Process diagram of split-step scheme of the Navier-Stokes equation . 22

7 LEV vorticity contour at mid-span with the Q-criterion (black line) defining the boundaries of identified vortices at $45^{\circ}$. . . . . . . . 26

8 A structured grid meshed between an annulus and square. . . . . . . 27

$9 \quad$ A multiblocked structured grid. . . . . . . . . . . . . . 28

10 Unstructured annulus and square mesh [4] . . . . . . . . . . . . 29

11 Cartesian grid of annulus and square. . . . . . . . . . . . . . . 30

12 Example of Overlapping Structured Grid system $[5,6]$. . . . . . . . 31 
13 Component grids a Cartesian grid, G1, and a body-fitted structured grid around an annulus, G2. [6,7] . . . . . . . . . . . . . 33

14 Identifying unused, and interpolation points $[6,7] \ldots 34$

15 Completed overlapping grid system $[6,7] \ldots$. . . . . . . . . . . 34

16 Trimmed overlapping grid $[6,7] \ldots \ldots$. . . . . . . . . . 35

17 Overlapping grid layout used for code validation . . . . . . . . . . 37

18 Comparison of lift coefficients of a SD7003 airfoil undergoing a sinusoidal plunge motion. . . . . . . . . . . . . . . . . . . . . . . . . . . . 39

$192 \mathrm{D}$ lift and drag coefficients of a flat plate pitching up around the leading edge at $\mathrm{K}=0.2$ and $\mathrm{Re}=20,000$. . . . . . . . . . . . . . . . . 40

20 Flat plate profile with a chord $(c)$ of one, and $5 \%$ chord thickness $(t) \quad 42$

21 Planform of the test wing gemoetries where $z$ is the span length. . . . 42

22 (Left) 2D body fitted grid. (Right) 3D composite grid. . . . . . . . . 43

23 (Left) 2D and (right) 3D grid domains. . . . . . . . . . . . . 43

24 Aerodynamic forces for the grid sensitivity analysis of a pitching wing of $\mathrm{AR}=2, \mathrm{~K}=0.2$, and $\mathrm{Re}=500$. . . . . . . . . . . . . . 46

$25 \mathrm{U}$ and $\mathrm{V}$ velocity distributions quarter chord behind the airfoil at $45^{\circ} .47$

26 Reynolds number sensitivity of the $2 \mathrm{D} \mathrm{K}=0.2$ pitch-up case tested at Reynolds numbers of 500, 5000, 10,000, and 20,000 compared to Granlund et al. $(\mathrm{Re}=20,000)[8]$

27 Change in vorticity due to Reynolds number sensitivity of the 2D $\mathrm{K}=0.2$ pitch-up case tested at Reynolds numbers of 500, 5000, 10,000,

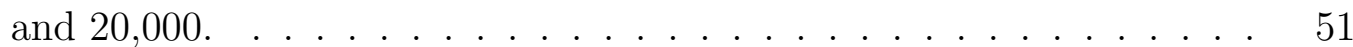

28 Coefficient of lift (solid) and drag (dotted) for the pitch-up cases at their respective instantaneous angles of attack. . . . . . . . . . . . . 52

29 Vorticity contours at differing pitch rates and angles at pitch-up with a Reynolds number of 500. . . . . . . . . . . . . . 
30 (Top) Coefficient of lift and (Bottom) coefficient of drag for perching cases normalized by initial instantaneous velocity (solid) and initial flow velocity (dotted) at their respective instantaneous angles of attack. 54

31 Comparison of lift and drag coefficients at different aspect ratios. (Pitchup, $\mathrm{Re}=500) . \ldots \ldots \ldots \ldots \ldots \ldots$

32 Comparison of lift and drag coefficients at different aspect ratios. (Perching, $\mathrm{Re}=500) \ldots \ldots \ldots \ldots \ldots$

33 Vorticity contours at the mid-span of pitching plates with varying aspect ratios (pitching case, $\mathrm{K}=0.2, \mathrm{AoA}=45^{\circ}$ ) . . . . . . . . . 59

34 Comparison of the LEV vorticity contours at mid-span with the Qcriterion defining the boundaries of identified vortices. Snapshots were taken at $45^{\circ} \ldots \ldots \ldots \ldots \ldots \ldots$

35 Comparison of LEV vorticity during the pitch-up motion of the $A R=4$ wing for $K=0.1$ and 0.2 with the Q-criterion defining the boundaries of identified vortices. . . . . . . . . . . . . . . .

36 Comparison of LEV vorticity during the perching motion of the $A R=4$ wing for $K=0.1$ and 0.2 with the Q-criterion defining the boundaries of identified vortices. . . . . . . . . . . . . . . . .

37 The normal force coefficient distribution plotted across the span of varying aspect ratios and angles of attack for the $K=0.2$ pitch-up. . .

38 Iso-surfaces of the Q-criterion $(Q=5)$ at discrete angles of attack of each aspect ratio at $K=0.2$. Views are in the upstream direction looking at the trailing edge of the wing. . . . . . . . . . . . . . 67

39 The normal force coefficient distribution plotted across the span of varying aspect ratios and angles of attack for the $K=0.1$ pitch-up. . .

40 Iso-surfaces of the Q-criterion $(Q=5)$ at discrete angles of attack of each aspect ratio at $K=0.1$. Views are in the upstream direction looking at the trailing edge of the wing. . . . . . . . . . . 
41 Normalized normal force distributions along the span of various aspect ratios for the pitch-up (solid) and perching (dashed) cases at $\mathrm{K}=0.2$. $\quad 72$

42 Normalized normal force distributions along the span of various aspect ratios for the pitch-up (solid) and perching (dashed) cases at $K=0.1$. $\quad 73$

43 The normal force coefficient distribution plotted across the span of varying aspect ratios and angles of attack for the $K=0.2$ perching. . . 74

44 The normal force coefficient distribution plotted across the span of varying aspect ratios and angles of attack for the $K=0.1$ perching. . . 75

45 Iso-surfaces of the Q-criterion $(Q=1)$ at discrete angles of attack of each aspect ratio at $K=0.1$. Views are in the upstream direction looking at the trailing edge of the wing. . . . . . . . . . . . . 77

46 Iso-surfaces of the Q-criterion $(Q=1)$ at discrete angles of attack of each aspect ratio at $K=0.2$. Views are in the upstream direction looking at the trailing edge of the wing. . . . . . . . . . .

47 (a) Lift (solid) and noncirculatory (dotted) versus angle of attack of three pitch rates. (b) Resultant lift coefficient with noncirculatory component removed. . . . . . . . . . . . . . . . .

48 (a) Actual lift (solid) and circulatory (dotted) coefficients versus angle of attack of three pitch rates. (b) Actual and circulation lift coefficients with noncirculatory component removed. . . . . . . . . . . . .

49 Coefficient of lift due to circulation divided by pitch rate versus the nondimensionalized time since the start of the pitch-up motion. . . . 88

50 The area occupied by the vortex is assumed to be a perfect circle . . 89

51 (a) Radius of LEV versus angle of attack of three pitch rates. (b) Radius of LEV versus circulation lift coefficient. . . . . . . . . . . . . 91

52 Snapshots of the Q-Criterion $(1<\mathrm{Q}<25)$ when $r_{L E V}=0.2$ at three different pitch rates. . . . . . . . . . . . . . . . 
53 Total, LEV, and shear quarter and mid-span circulation for each aspect ratio for $K=0.1$. Dotted and solid lines represent $z / b=0.25$ and $z / b=0.5$ respectively. . . . . . . . . . . . . . .

54 Total, LEV, and shear quarter and mid-span circulation for each aspect ratio for $K=0.2$. Dotted and solid lines represent $z / b=0.25$ and $z / b=0.5$ respectively. . . . . . . . . . . . . . . .

55 Total, LEV, and shear circulation across the entire span for each aspect ratio and $K$. Solid and dotted lines represent $K=0.1$ and 0.2

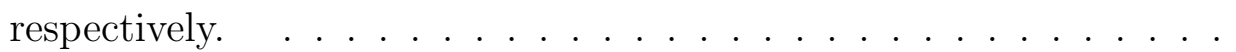

56 Total, LEV, and shear quarter and mid-span circulation for each aspect ratio for $K=0.1$. Dotted and solid lines represent $z / b=0.25$ and $z / b=0.5$ respectively. . . . . . . . . . . . . . . .

57 Total, LEV, and shear quarter and mid-span circulation for each aspect ratio for $K=0.2$. Dotted and solid lines represent $z / b=0.25$ and $z / b=0.5$ respectively. . . . . . . . . . . . . . . .

58 Total, LEV, and shear circulation across the entire span for each aspect ratio and $K$. Solid and dotted lines represent $K=0.1$ and 0.2 respectively.

59 (Left) Contour of spanwise $\Gamma_{L E V}$ through entire pitching motion for the $A R=1$ wing at both tested $K$ values. (Right) Iso-surface contours where the $Q=5$ for both $K$ values at $30^{\circ}, 45^{\circ}$, and $60^{\circ} \ldots \ldots$

60 (Left) Contour of spanwise $\Gamma_{L E V}$ through entire pitching motion for the $A R=2$ wing at both tested $K$ values. (Right) Iso-surface contours where the $Q=5$ for both $K$ values at $30^{\circ}, 45^{\circ}$, and $60^{\circ} \ldots$. . . .

61 (Left) Contour of spanwise $\Gamma_{L E V}$ through entire pitching motion for the $A R=4$ wing at both tested $K$ values. (Right) Iso-surface contours where the $Q=5$ for both $K$ values at $30^{\circ}, 45^{\circ}$, and $60^{\circ} \ldots$. . . . . 102 
62 (Left) Contour of spanwise $\Gamma_{L E V}$ through entire perching motion for the $A R=1$ wing at both tested $K$ values. (Right) Iso-surface contours where the $Q=1$ for both $K$ values at $30^{\circ}, 45^{\circ}$, and $60^{\circ} \ldots$. . . . 103

63 (Left) Contour of spanwise $\Gamma_{L E V}$ through entire perching motion for the $A R=2$ wing at both tested $K$ values. (Right) Iso-surface contours where the $Q=1$ for both $K$ values at $30^{\circ}, 45^{\circ}$, and $60^{\circ} \ldots \ldots 4$

64 (Left) Contour of spanwise $\Gamma_{L E V}$ through entire perching motion for the $A R=4$ wing at both tested $K$ values. (Right) Iso-surface contours where the $Q=1$ for both $K$ values at $30^{\circ}, 45^{\circ}$, and $60^{\circ} \ldots$. . . . . 105

65 Uniform Grid Refinement Around Airfoil . . . . . . . . . . . . . . . . 118

66 Static lift, drag, and moment coefficients at an angle of $13.04^{\circ}$. . . . . 120

67 Changes in vorticity with grid resolution of a steady state angle of attack at $13.04^{\circ} \ldots \ldots \ldots \ldots \ldots$

68 Lift, and drag coefficient variation due to selected spatial resolution of a pitching airfoil between $13 \pm 4^{\circ}$. . . . . . . . . . . . . . . . . 122

69 Lift, and drag coefficient variation due to selected temporal resolution of a pitching airfoil between $13 \pm 4^{\circ}$.

70 Lift, and drag coefficient of a pitching airfoil between $13 \pm 4^{\circ}$ at $\Delta t=$ $0.05,0.1$, and $0.2 \ldots \ldots \ldots \ldots \ldots \ldots$. . . . . . . . . . . . . . . 124

71 Computed lift and drag coefficient errors due to selected spatial and temporal resolutions. . . . . . . . . . . . . . . . . . . . 125

72 Feature-based adaptation process . . . . . . . . . . . 126

73 The initial, $4^{\text {th }}$, and $6^{\text {th }}$ mesh adaptation of the selected $h_{4}$ grid. . . . 128

74 Node increase with adaptation at each iteration . . . . . . . . . . . 128

75 Change in cell volume ratio $\left(R_{c v}\right)$ between consecutive adaptation iteration steps. (Red) $R_{c v}>1$, (Green) $R_{c v} \approx 1$, (Blue) $R_{c v}<1$. . . . 130

76 Cycle averaged vorticity magnitude of the initial, $2^{\text {nd }}, 4^{\text {th }}$, and $6^{\text {th }}$ adapted meshes. . . . . . . . . . . . . . . . . . 132 
77 Coarsening of mesh and changes in vorticity close to the airfoil surface due to mesh adaptation. . . . . . . . . . . . . . . . . 133

78 Instantaneous lift, drag, and moment coefficients of the initial, $2^{\text {nd }}, 4^{\text {th }}$, and $6^{\text {th }}$ adapted meshes. . . . . . . . . . . . . . 134

79 Average and error of lift, drag, and moment coefficients of adapted grids136 


\section{CHAPTER I}

\section{INTRODUCTION}

\section{A. Lift Generation of Unsteady Wings}

Interest in understanding the perching maneuver used in bird and insect flight stems from the growing interest in Micro-Air-Vehicles (MAVs) research. MAVs are small aerial vehicles with a typical wing span of less than $15 \mathrm{~cm}$ and have a mass less than 90 grams. Their flight speed usually ranges from $2 \mathrm{~m} / \mathrm{s}$ to $10 \mathrm{~m} / \mathrm{s}$. The development of MAVs is of great interest to both military and civilian applications due to their versatile nature in various scenarios such as reconnaissance, surveillance, targeting, search and rescue, and biochemical sensing in confined or otherwise hazardous conditions. Their applications are similar to their larger cousin, the unmanned aerial vehicle (UAV), but MAVs have much smaller sizes and much lower cost. As an example, the General Atomics Aeronautical Systems Predator ${ }^{1}$ UAV has a cruise speed about $35 \mathrm{~m} / \mathrm{s}$, a wing span of $14.8 \mathrm{~m}$, and cost upwards of approximately $\$ 10$ million. The goal of MAV design is to fit the capabilities of a UAV into a far smaller aircraft.

While there is large potential for the use of MAVs, there are several problems that must be overcome due to their small size and low flight speed. The low Reynolds number presents new aerodynamic challenges in efficient lift generation. Due to MAVs slow flight speed, the chord Reynolds number across the wing can be low, $O\left(10^{2}\right.$ $10^{4}$ ), where the flow across the wing remains laminar. Current MAV designs rely on conventional streamlined airfoils for lift generation; while conventional airfoils are

\footnotetext{
${ }^{1}$ www.ga-asi.com
} 


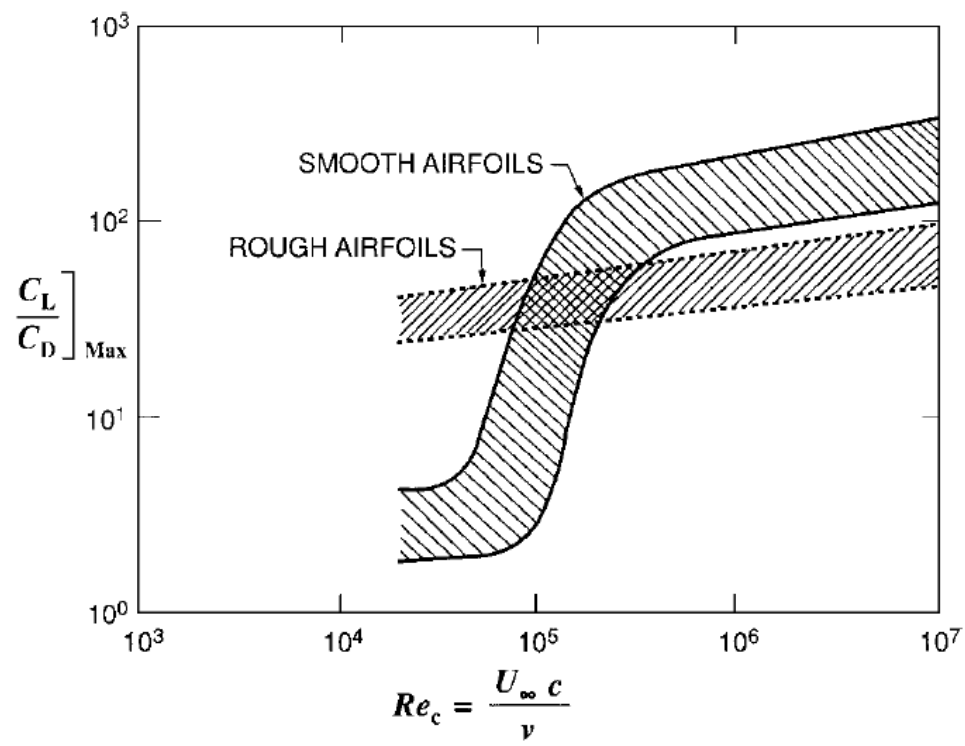

Figure 1: Gliding ratio as a function of chord Reynolds number [2]

designed to operate at much higher Reynolds numbers greater than $O\left(10^{5}\right)$. The performance of smooth airfoils deteriorates once the Reynolds number drops below $O\left(10^{5}\right)$. Figure 1 is a reproduction of a plot from McMasters and Henderson [2] where the maximum lift-to-drag ratios of airfoils are plotted as a function of Reynolds number. The lift-to-drag ratio is a measurement of the effectiveness of an airfoil which is proportional to the gliding ratio and climbing ability of the airfoil [9]. For large aircraft the boundary layer usually transitions to turbulent flow before separation. This is due to turbulent flow being able to stay attached through adverse pressure gradients as compared to laminar flow. Since laminar flow cannot resist adverse pressure gradients along the wing's surface, flow can separate leading to premature stall. This results in a significant decrease in the lift, and increase in the drag [9]. Thus static airfoils are only able to operate at very low angles of attack to avoid stall. In order to improve upon a MAVs stability and maneuverability, low Reynolds number flow separation must be controlled or mitigated.

The nature progression of MAV development has seen the phasing out of fixed wing MAVs in favor of flapping wings. Flapping wings have the potential 
to provide superior lift generation, excellent maneuverability, and robust gust resistance for MAVs (micro-air-vehicle) operating at low Reynolds numbers $\left(O\left(10^{3}-\right.\right.$ $\left.\left.10^{4}\right)\right) .[10,11,12,13,14]$ The enhanced lift generation of a flapping wing is in part due to the creation of a leading-edge-vortex (LEV) over the surface of the wing. The initial proximity of the LEV to the wing generates high lift as the vortex convects in the chord-wise direction. $[15,16,17]$ This high lift generation mechanism is similar to dynamic stall, which refers to the unsteady process of lift generation when the lift on a rapidly pitched wing continues to increase even after the angle of attack passes the static stall angle. Dynamic stall is frequently encountered in helicopters, turbo-machinery, wind turbines, and nature fliers. [18]

Carr [19] originally presented the prominent flow features within a full dynamic stall cycle oscillation which are illustrated in figure 2. The dynamic stall cycle can be broken into a set of discrete steps: 1) the airfoil begins to pitch and a leading edge vortex forms, 2) the vortex convects along the chord of the airfoil, 3) the vortex separates leading to the onset of stall, 4) flow becomes separated and airfoil pitches downward, and 5) flow reattaches and lift is recovered. During steps (1) and (2), large lift forces are experienced due to the presence of the vortex. At the onset of stall, the vortex sheds from the airfoil, resulting in a decrease in lift and flow separation. Finally, flow remains detached until the angle of attack decreases enough to allow for flow reattachment. The lift benefits of a vortex generated by this motion are not limited to cyclic motions.

Shih et al. [20] found a similar set of flow features during a single pitch-up sequence. Steps 1) through 3) remained largely the same, but since the airfoil does not pitch back down the flow never reattaches. Thus at 4) flow separation or bluff body shedding begins. Between the two dynamic stall motion sets, one can conclude the lift forces experienced, are largely determined by the presence of the generated vortex. It has been widely concluded $[18,16,21,22,23,20,8,24,25]$ that increasing pitch rate, and peak angle of attack increases the strength of the vortex, thus increasing lift 


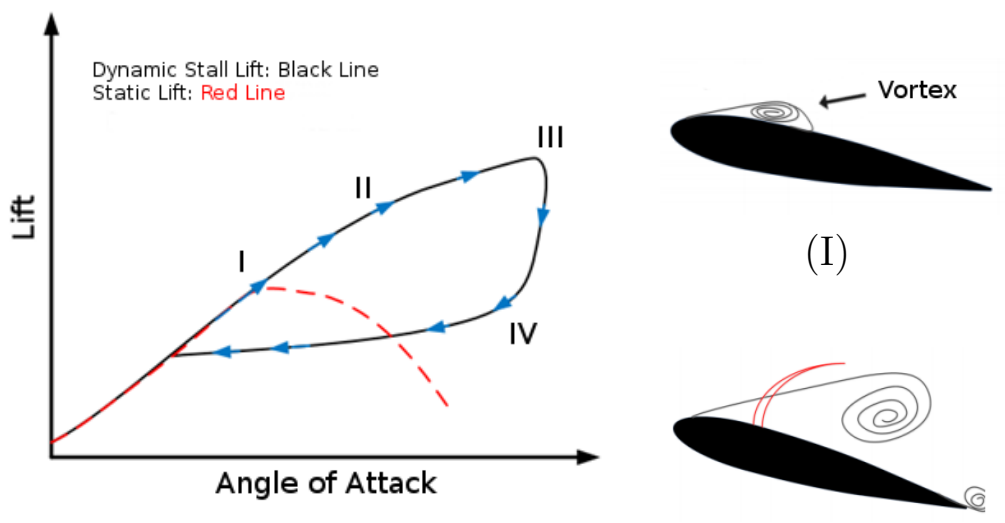

(III)

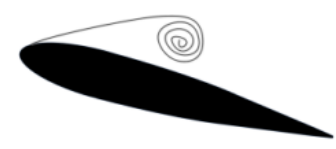

(II)

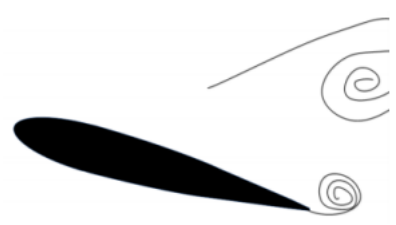

(IV)

Figure 2: Illustration of the key features in a dynamic stall phenomenon. (Left) Comparison of lift curves between static stall and dynamic stall; (Right) Evolution of dynamic stall vortex. (I) Start of the leading edge separation. (II) Vortex rollup; (III) Stall onset and formation of a trailing edge vortex; (IV) Detachment of the dynamic stall vortex, and the sudden lift loss.

for both motion sets. Both motions have been thoroughly tested by the referenced authors at moderate rates and angle amplitudes. Regardless of the pitching motion, the dynamic stall process is largely the same.

Because of its scientific merits and practical significance, dynamic stall related problems have been studied both numerically and experimentally. [26, 27, 28, 29, $30,12]$ Most numerical studies have investigated the phenomena as a prominently 2D problem due to the immense computational resources required for a 3D study [31, 19, 18, 13]. Numerical studies with complicated flapping kinematics have been conducted at low Reynolds numbers; however, the focus was on force and moment development instead of dynamic stall phenomenon. [29, 32] For a dynamic stall problem, high Reynolds number $\left(R e>10^{6}\right)$ 3D experiments have been conducted by Piziali [33] and Tang \& Dowell [34] for helicopter applications, but low Reynolds number $\left(R e \approx 10^{3}-10^{4}\right) 3 \mathrm{D}$ experiments and simulations are sparse. [35, 36, 37, 8, 30, 38] Since MAVs employ low aspect ratio wings, in order for the dynamic stall research to be applicable to MAVs, dynamic stall must be investigated as a 3D problem at low Reynolds numbers. This in essences summarizes the issues faced in current MAV-wing 
research undergoing a dynamic stall related motion. The few studies have examined the Reynolds number regime of MAVs have found that changes in Reynolds number, rate of change of angle of attack and aspect ratio can all alter the forces and flow field around a wing.

Experimental and numerical studies examining a 2D pitch-up wing have shown that the Reynolds number plays an important role in determining the lift, drag, moment, and the resulting LEV. Eldredge[39], Garmann \& Visbal[40], and Ol et al. [41] observed increases in lift and drag of a pitching wing as the Reynolds number increased from 10,000 to 20,000. Inspections of the flow field revealed the LEV became compact and interior vorticity increased. They concluded the change in the LEV was responsible for the increase in aerodynamic forces.

The study of finite aspect ratio wings at low Reynolds numbers is an area that has not been well explored, even more so with the dynamic stall phenomena. Recent studies on static wings at low Reynolds numbers $\left(O\left(10^{4}\right)\right)$ have concluded that the aspect ratio significantly affects the performance of the wing. [42, 43, 44] For flapping wings, aspect ratio has been found to influence the flow field. Granlund et al. [30] experimentally found that a finite aspect ratio pitch-up wing had a less defined LEV structure when compared to a 2D case with the same operating conditions. Yilmaz \& Rockwell[38] experimentally studied a 3D pitch-up wing and found a spanwise velocity induced by tip vortices altered the LEV development. This phenomenon was also seen in Coton \& Galbraith [36] and Spentzos et al.[37].

The pitch rate of the dynamic stall motion has been known to influence the maximum achievable lift and stall angle. [31, 16, 19]. Granlund et al. [8] experimentally surveyed several pitching rates of a nominally 2D airfoil undergoing a single pitch-up maneuver at a Reynolds number of 20,000. They found that even at a low pitch rate, lift would be higher than its corresponding static stall value. Interestingly, the slope of the lift curve changed little with the pitch rate except when the reduced pitch rate was greater than 0.2. Later, Granlund et al. [30] surveyed reduced pitch 
rates for a 3D wing and found that increased pitch rates caused tip vortices to develop at higher angles than LEVs.

\section{Perching Wings}

There exists a small subset of MAV pitch-up wing research investigating the role of the perching maneuver commonly seen in nature fliers. The perching maneuver is a simplified set of kinematics that mimic motions birds or insects undergo to land at a point in space. This motion consists of a high angle of attack pitch-up motion, with a simultaneous deceleration of the wing to a zero velocity state. Normally the maneuver is complex and it involves elastic deformation of the wing, fast variation of the angle of attack, complicated wing kinematics, and real time adjustment of trajectory of the body; however, the prominent aerodynamic features are very similar to those observed in a classical pitch-up wing problem. Though the real perching motion and the pitch-up problem are different because the former includes deceleration in the direction of motion and experiences zero velocity at the end of the maneuver, they both have common unsteady flow phenomena such as massive flow separation, deep stall, and the formation and shedding of leading edge and trailing edge vortices all seen in low Reynolds number flow regimes. The challenges in studying the perching maneuver can be further exacerbated by the low aspect ratio wings and low Reynolds number MAVs operate at similar to the study of a pitch-up motion.

Granlund et al. [8] experimentally studied the aerodynamics of a perching wing mounted wall-to-wall in a water tunnel at a Reynolds number of 20,000. The wing had a rectangular planform and with a cross section is a flat plate with round leading and trailing edges. Their experiment was intended to approximate a two-dimensional (2D) model. A survey of the pitching rate and pivot point was conducted as the wing was linearly pitched up from $0^{\circ}$ to $90^{\circ}$. They found that even at a very low reduced pitching rate $(K=0.0025)$ lift would overshot beyond static stall, similar to dynamic stall. The slope of the lift curve changed little with the reduced pitching rate except 
at a high reduced pitching rates of 0.2. They observed that wing deceleration had significant impact on the force history when the pivot point was fore of the mid-chord but the impact is negligible when the pivot point was aft of mid-chord.

Later Granlund et al. [24] extended their early experimental study to include the aspect ratio effect. A rectangular wing with a flat plate cross sectional profile and an aspect ratio of 2 was used. They studied the effects of pitch rate and pivot point and compared with their previously studied wall-to-wall model. Comparing to the wall-to-wall case, the finite aspect ratio wing had a less coherent leading edge vortex structure. Tip vortices were seen to develop slower than the leading edge vortex. The tip vortices would remain attached to the wing longer than the leading edge vortex, suggesting tip vortices attenuate aerodynamic forces on low aspect ratio wings. Peaks in the lift coefficient were found to increase asymptotically with pitch rate; however, the occurrence of peak lift is delayed with the increase of pitch rate. They found the lift forces due to the pitching of the wing were linearly additive to the measured lift.

While the depth of research that exists for the examination of wings undergoing the perching motion is limited, one can draw several parallels to the pitch-up motion. Due to the perching motion being a compounded set of kinematics that incorporate the pitch-up motion, many insights can be drawn by simulating various wing aspect ratios undergoing pitch-up and be extended to the perching maneuver. Thus this brings us to primary purpose of this dissertation. In this dissertation, a parametric study on pitching and perching wings was conducted to fill in the gaps of knowledge that exist in the low Reynolds number regime of MAV flight.

\section{B. Objectives and Organization}

The objective of this dissertation is to numerically investigate the flow phenomena of flat plate wings with various aspect ratios undergoing a pitch-up and perching motion at a Reynolds number of 500. In this dissertation, both motions will be investigated numerically using $2 \mathrm{D}$ and $3 \mathrm{D}$ numerical methods analyzing the simulated 
aerodynamic forces, and the development of the LEV. Special attention will be paid to the development of circulation in the LEV to attempt to better quantify the changes in kinematics and geometry have of the development of the LEV. This will be done by directly calculating the circulation in the flow.

Before examining the circulation of the LEV directly, an understanding of several factors must be taken into account: 1) Reynolds number, 2) the kinematic motion, and 3) aspect ratio effects. This will be done by first examining the lift and drag forces produced by the $2 \mathrm{D}$ and $3 \mathrm{D}$ wings.

For the 2D study, reduced pitch rates of $0.05,0.1$, and 0.2 will be examined at four Reynolds numbers between 500 and 20,000. The three selected reduced pitch rates stem from published experimental data, and have been tested by other authors. The lower Reynolds number value is of interest for being in the lower range of MAV flight.

The 3D study will use three finite aspect ratio wings of 1, 2 and 4 pitching at 0.1 and 0.2 . These aspect ratios are approximately the equivalent size of small to large insect wings. For both $2 \mathrm{D}$ and $3 \mathrm{D}$ cases, the wing will pitch from $0^{\circ}$ to $90^{\circ}$ for the pitching and perching simulations. Due to the similarities in flow structure and force coefficients, the pitch-up motion is primarily analyzed for the majoring of the dissertation. The investigation is broken into several sections to address the following topics:

Reynolds number effects on 2D pitching wings - Due to experimental studies being conducted at higher Reynolds numbers, the effect of Reynolds number must first be quantified to justify numerical results at a lower Reynolds number. A pitch-up motion was tested at several Reynolds numbers and compared to experimental data.

Kinematic effects on pitching and perching wings - Three reduced pitch rates were selected in order to investigate the effect of kinematics on the pitching and perching maneuvers on the resultant lift and drag. 
Aspect ratio effects on pitching and perching - To investigate three dimensional effects on the pitching and perching motions, three aspect ratios were selected and compared. In combination with aspect ratio, two reduced pitching rates were used to quantify the resultant forces, and force distribution across each wing.

Circulatory and noncirculatory analysis of a pitching wings - Forces generated by a pitching wing are not entirely due to lift and drag. Using the Theodorsen model, forces on a 2D wing were separated into lift produced by the LEV (circulatory forces) and due to the wing motion (noncirculatory forces). In conjunction with this investigation, the radius of the LEV was computed to understand how circulation and LEV radius are related.

Circulation development of a 3D LEV - The 2D circulation study was continued with the finite aspect ratio wings; however only the circulation of the LEV was analyzed. The resulting analysis gave a view into the distribution of circulation across a wing undergoing both the pitch-up and perching motions. While the motions are similar, circulation distribution differs between the two.

Feature based adaptation of dynamic stall - Work interrelated to the simulation of dynamic stall was also conduced with NASA Langley. Inadequate grid resolution can alter the resulting flow field in the wake of a dynamic stall airfoil. Feature based methods were investigated for their effectiveness in reducing spatial error due to user generated meshes. 


\section{CHAPTER II}

\section{PERCHING MANEUVER KINEMATICS}

\section{A. Perching Simplification}

Data from bird flights provide insightful information on the kinematic motions of zero length landing (also known as perching). Berg and Biewener [3] studied the flight behaviors of a Columba Livia, pigeon, during takeoff, standard mid-flight, and perch landing. The pigeon's wing was broken into three regions (arm wing, proximal hand wing, and distal hand wing), and the average angle from the horizontal plane of each section was measured at selected wing beats. Figure 3 plots the measured averaged angles of the wing during various flight modes. The mid-flight angle was not plotted, but was noted to be approximately $15^{\circ}$ from the horizontal axis.

From this plot, ignoring the flapping motion, it is possible to break down how the wings pitch-up during perch landing. The wings are initially at an averaged cruising angle of $15^{\circ}$. During the first wing beat, location -3 , the wings are pitched up to approximately $30^{\circ}$, during the second wing beat, the wings are pitched up to

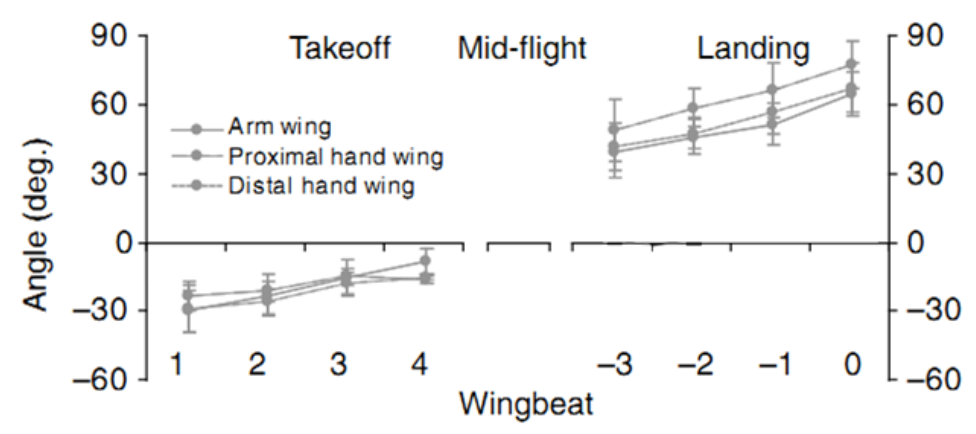

Figure 3: Angle from the horizontal plane at three different locations along a pigeon (Columba Livia) wing during three different flight modes. [3] 
about $50^{\circ}$ at -2 , during the third wing beat, $60^{\circ}$ at -1 , and during the last beat, $80^{\circ}$ at 0. These measurements provide some basis to justify simplifying a complex perching motion into a simple pitch-up problem. It should noted here that in real perching the wing experiences multiple flapping beats and in the simplified pitch-up problem the wing will monotonically pitch up.

\section{B. Pitching Motion}

To avoid sudden start and stop of the pitch-up motion, which can cause high acceleration related forces and noise in the simulation, smoothing transitions are applied at the beginning and ending of the pitching motion. A hyperbolic-cosine function developed by Eldredge et al. [45] was originally for a pitch-up, hold, and pitch-down kinematic study. The function smooths the higher derivatives of the motion to minimize acceleration effects. The smoothing function $G(t)$ was defined as

$$
\begin{gathered}
G(t)=\ln \left[\frac{\cosh \left(a U_{1}\left(t-t_{1}\right) / c\right) \cosh \left(a U_{1}\left(t-t_{4}\right) / c\right)}{\cosh \left(a U_{1}\left(t-t_{2}\right) / c\right) \cosh \left(a U_{1}\left(t-t_{3}\right) / c\right)}\right] \\
\alpha(t)=\alpha_{\max } \frac{G(t)}{\max (G(t))}
\end{gathered}
$$

where $a$ is a user defined value that controls the sharpness of the function, which must be greater than 1, $c$ is the chord, $\alpha$ is the angle of attack for the wing with respect to time, $U$ is the free stream velocity, and $\alpha_{\max }$ is the maximum angle of attack. The time constants $t_{1}$ through $t_{4}$ are characteristic times chosen by the user to fit the prescribed motion. $t_{1}$ is the beginning of the pitch-up motion, $t_{2}$ is the time at the end of the pitch-up, and the beginning of the hold, $t_{3}$ began the pitch down, and $t_{4}$ is the end of the motion.

To apply Eqn. 2.1 to the perching kinematics, an adjustment must be made. Since $t_{3}$ and $t_{4}$ periods will never be reached, due to the wing pitching up only, the values can be dropped from the equation, and the new function becomes: 


$$
G(t)=\ln \left[\frac{\cosh \left(a U_{1}\left(t-t_{1}\right) / c\right)}{\cosh \left(a U_{1}\left(t-t_{2}\right) / c\right)}\right]+\frac{a U_{1}\left(t_{2}-t_{1}\right)}{c}
$$

The values of $t_{1}$ and $t_{2}$ can be determined from the desired reduced pitching rate, $K$, which follows below. In this study, $K$ is equal to either $0.05,0.1$, or 0.2 .

$$
K=\frac{c \dot{\alpha}}{2 U}=\frac{c}{2 U} \frac{\alpha_{2}-\alpha_{1}}{t_{2}-t_{1}}
$$

Where $c$ is the chord length, $\dot{\alpha}$ is the pitch rate, and $U$ is the free stream velocity. This definition is consistent with the definition used by Granlund et al $[8,24]$ which differs from Visbal and Shang [22].

An example of the pitch-up kinematics is plotted in figure 4 where $t_{1}$ and $t_{2}$ were calculated from $K=0.1$ and $K=0.2$ and $a$ varies from 1 to 5 . As $a$ increases, the pitching kinematics transitions from having smooth start and finish motions to a sharp ramp function. It is important to control $a$ as the duration of the acceleration of the pitch is directly affected. This allows for effects due to rotational acceleration to be minimized such as an impulsively started rotation. This can cause issues in both experimental and numerical studies. Additionally, the start of the motion influences the lift coefficient. The importance of this will be discussed later in Chapter VII.

For this dissertation $a$ is always equal to 5 , and the wing pitches about the quarter-chord location $(x / c=0.25)$. This pivot point was chosen to coincide with existing published research at the higher Reynolds number. Granlund et al.[8] offers a summary of the effects of moving the pivot point. Moving the pivot point aft of mid-chord reduces the lift generated by the pitching motion.

\section{Deceleration}

As stated previously, the perching motion is simplified into a pitch-up motion coupled with a simultaneous deceleration of the wing. Here the deceleration of the wing is in the horizontal direction and is handled similarly to the pitch-up motion. 

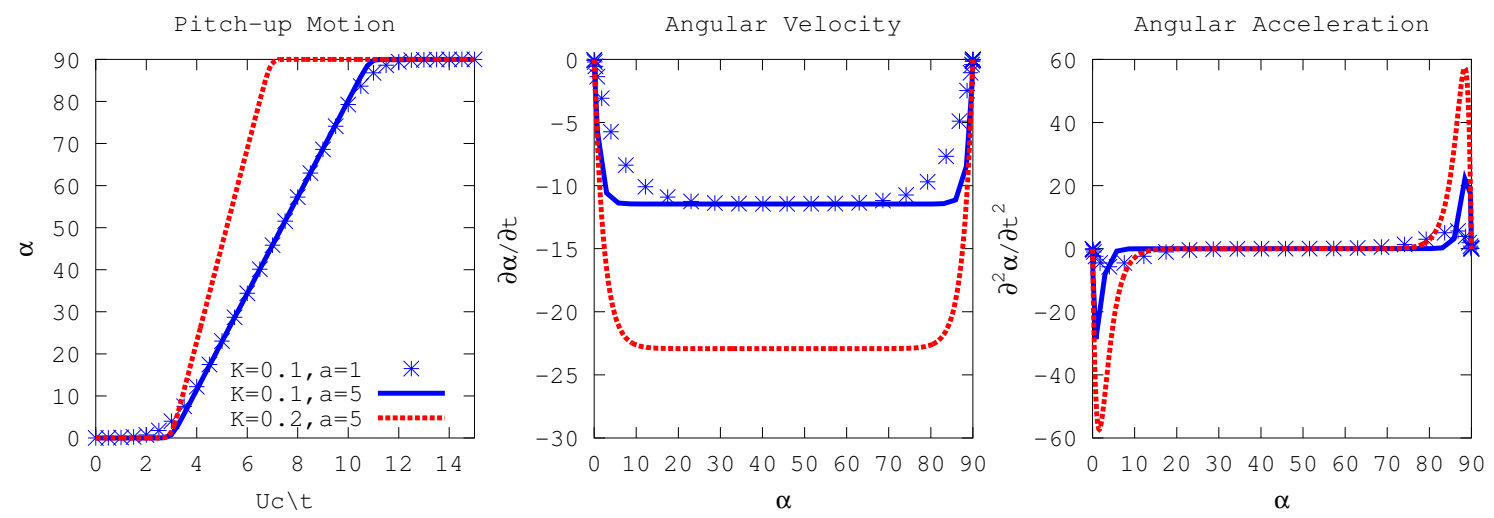

Figure 4: Impact of the smoothing parameter and $K$ on the pitch-up kinematics. (Left) Change in angle of attack, (middle) angular velocity, and (right) acceleration of the wing.

The hyperbolic-cosine function defined for the pitching motion, is again used, and modified slightly by switching the locations of $t_{1}$ and $t_{2}$ to handle the initial non-zero velocity.

$$
\begin{gathered}
R(t)=\ln \left[\frac{\cosh \left(a U_{1}\left(t-t_{1}\right) / c\right)}{\cosh \left(a U_{1}\left(t-t_{2}\right) / c\right)}\right]+\frac{a U_{1}\left(t_{2}-t_{1}\right)}{c} \\
V(t)=V_{\max } \frac{R(t)}{\max (R(t))}
\end{gathered}
$$

where $V_{\max }$ is the initial velocity of the wing. Much like the pitching motion, the deceleration profile can be controlled through the user defined $a$ variable as shown in figure 5. Again, for this dissertation, $a$ is held equal to that of the pitching motion of 5. It is currently unclear as to how different deceleration profiles affect the simulated results. There has been no study on this to the date. 

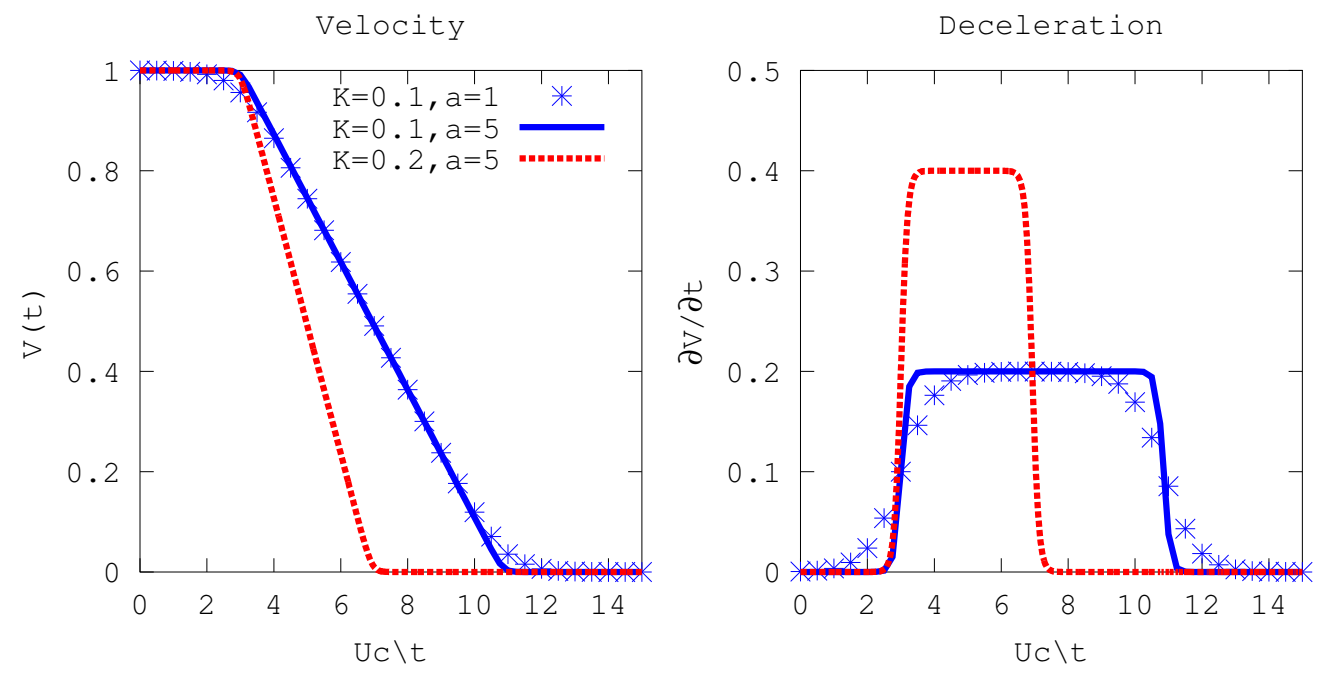

Figure 5: Declaration profile using smoothing function. (Left) Velocity (units per second) with respect to time (seconds). (Right) Declaration with respect to time. 


\section{CHAPTER III}

\section{NUMERICAL METHOD}

\section{A. Navier-Stokes System of Equations}

In this chapter, the governing equations of the physics involved in the numerical

simulation will be introduced. First the governing equations of fluid dynamics will be shown and then how they can be derived and adapted for incompressible viscous flow. The equations governing fluid flow can be derived from the equations of the conservation of mass, momentum, and energy [46]. These equations can be written in differential form as

$$
\begin{gathered}
\frac{\partial \rho}{\partial t}+\nabla \cdot(\rho \vec{u})=0 \\
\frac{\partial(\rho \vec{u})}{\partial t}+\nabla \cdot(\rho \vec{u} \vec{u})=-\nabla p+\nabla \cdot \tau \\
\frac{\partial\left(\rho e_{t}\right)}{\partial t}+\nabla \cdot\left(\rho e_{t} \vec{u}\right)=-k \nabla \cdot \nabla T-\nabla \rho \cdot \vec{u}+(\nabla \cdot \tau) \cdot \vec{u}
\end{gathered}
$$

Where $\rho$ is the density, $t$ is the time, is the flow field velocity vector, $e_{t}$ is the total internal energy, $k$ is the thermal conductivity, $T$ is the temperature, and $\tau$ is the shear stress. The first of the three equations corresponds to the continuity equation of the conservation of mass law applied to a fluid passing through an infinitesimal fixed volume. The momentum equation is the second row, which is Newtons Second law applied to a fluid passing through an infinitesimal, fixed volume. Finally the third row, describes the Energy Equation in terms of the First Law of Thermodynamics. 
For low speed flows, it can be assumed that the fluids density is constant. The constant density can usually be assumed in almost all fluids, because their compressibility is neglected. For gasses with a Mach number lower than 0.3, the flow is usually considered to be incompressible. If the fluid is assumed to be incompressible and has constant viscosity, then the governing equations can be further simplified in to the following reduced set of equations where $\nu$ is the kinematic viscosity. $[46,6]$

$$
\begin{gathered}
\nabla \cdot \vec{u}=0 \\
\frac{\vec{u}}{\partial t}+\vec{u} \cdot \nabla \vec{u}=\frac{-\nabla p}{\rho}+v \nabla^{2} \vec{u}
\end{gathered}
$$

Further simplification can be made by nondimensionalizing the equations. Doing so will provide the conditions upon which dynamic similarity may be achieved for geometrically similar situations. Nondimensionalizing the equations also normalizes values so that they fall between zero and one which also reduces error by making the solution independent of any system of units [6]. This form of the Navier-Stokes equations can easily be rewritten in the form

$$
\begin{gathered}
\nabla \cdot \vec{u}=0 \\
\frac{\vec{u}}{\partial t}+\vec{u} \cdot \nabla \vec{u}=-\nabla p+\frac{1}{R e_{L}} \nabla^{2} \vec{u}
\end{gathered}
$$

where viscosity is replaced by the Reynolds number, $R e_{L}$, which is the nondimensionalization of the free stream density $\rho$, velocity $U_{\infty}$, viscosity $\mu$, and the reference length $L$. The equation for the Reynolds number is as follows

$$
R e_{L}=\frac{\rho_{\infty} U_{\infty} L}{\mu_{\infty}}
$$

Equation (3.8) can be further altered and expanded into Cartesian coordinates 


$$
\begin{gathered}
\frac{\partial u}{\partial x}+\frac{\partial v}{\partial y}+\frac{\partial w}{\partial z}=0 \\
\frac{\partial u}{\partial t}+u \frac{\partial u}{\partial x}+v \frac{\partial u}{\partial y}+w \frac{\partial u}{\partial z}=-\frac{\partial p}{\partial x}+\frac{1}{R e_{L}}\left(\frac{\partial^{2} u}{\partial x^{2}}+\frac{\partial^{2} u}{\partial y^{2}}+\frac{\partial^{2} u}{\partial z^{2}}\right) \\
\frac{\partial v}{\partial t}+v \frac{\partial v}{\partial x}+v \frac{\partial v}{\partial y}+w \frac{\partial v}{\partial z}=-\frac{\partial p}{\partial x}+\frac{1}{R e_{L}}\left(\frac{\partial^{2} v}{\partial x^{2}}+\frac{\partial^{2} v}{\partial y^{2}}+\frac{\partial^{2} v}{\partial z^{2}}\right) \\
\frac{\partial w}{\partial t}+w \frac{\partial w}{\partial x}+v \frac{\partial w}{\partial y}+w \frac{\partial w}{\partial z}=-\frac{\partial p}{\partial x}+\frac{1}{R e_{L}}\left(\frac{\partial^{2} w}{\partial x^{2}}+\frac{\partial^{2} w}{\partial y^{2}}+\frac{\partial^{2} w}{\partial z^{2}}\right)
\end{gathered}
$$

This form provides the simplest discretization and is widely used in numerical models for solving the incompressible Navier-Stokes equations [46]. For incompressible fluids flow with constant viscosity the Navier-Stokes equations can be decouple the energy and density equations. It is now possible to rewrite equation (3.7) into a compact vector form in Cartesian coordinates.

$$
\frac{\partial Q}{\partial t}+\frac{\partial E_{i}}{\partial x}+\frac{\partial F_{i}}{\partial y}+\frac{\partial G_{i}}{\partial z}=\frac{\partial E_{v}}{\partial x}+\frac{\partial F_{v}}{\partial y}+\frac{\partial G_{v}}{\partial z}
$$

where $Q$ is the original vector of spatial variables independent of energy and density

$$
Q=\left[\begin{array}{l}
0 \\
u \\
v \\
w
\end{array}\right]
$$

$E_{i}, F_{i}$, and $G_{i}$ are the vectors containing the inviscid fluxes in the x, y, and $\mathrm{z}$ directions and are given as 


$$
E_{i}=\left[\begin{array}{c}
u \\
u^{2}+p \\
u v \\
u w
\end{array}\right], F_{i}=\left[\begin{array}{c}
u \\
v u \\
v^{2}+p \\
v w
\end{array}\right], G_{i}=\left[\begin{array}{c}
u \\
w u \\
w v \\
w^{2}+p
\end{array}\right]
$$

$E_{v}, F_{v}$, and $G_{v}$, contain the viscous fluxes in the $\mathrm{x}, \mathrm{y}$, and $\mathrm{z}$ directions and they are given as

$$
E_{v}=\left[\begin{array}{c}
0 \\
\tau_{x x} \\
\tau_{x y} \\
\tau_{x z}
\end{array}\right], F_{v}=\left[\begin{array}{c}
0 \\
\tau_{y x} \\
\tau_{y y} \\
\tau_{y z}
\end{array}\right], G_{v}=\left[\begin{array}{c}
0 \\
\tau_{z x} \\
\tau_{z y} \\
\tau_{z z}
\end{array}\right]
$$

The viscous stress terms $\tau_{x x}, \tau_{y y}, \tau_{z z}, \tau_{x y}, \tau_{y x}, \tau_{x z}, \tau_{z x}, \tau_{y z}$, and $\tau_{z y}$ are also affected by the incompressible assumption. These are defined by the following relationships

$$
\begin{gathered}
\tau_{x x}=2 \mu\left(\frac{\partial u}{\partial x}\right) \\
\tau_{y y}=2 \mu\left(\frac{\partial v}{\partial y}\right) \\
\tau_{z z}=2 \mu\left(\frac{\partial w}{\partial z}\right) \\
\tau_{y x}=\tau_{x y}=\mu\left(\frac{\partial u}{\partial y}+\frac{\partial v}{\partial x}\right) \\
\tau_{z x}=\tau_{x z}=\mu\left(\frac{\partial u}{\partial z}+\frac{\partial w}{\partial x}\right) \\
\tau_{z y}=\tau_{y z}=\mu\left(\frac{\partial v}{\partial z}+\frac{\partial w}{\partial y}\right)
\end{gathered}
$$


The Navier-Stokes equations have been presented in terms of a Cartesian coordinate system. However, many applications require the equations to be expressed in a curvilinear coordinate system that allows the system of equations to be applied to a distorted region of physical space. This process will is not covered in this dissertation.

\section{B. Solving the Incompressible Navier Stokes Equations}

This section will give a brief discussion of the numerical method used to solve the governing equations outlined in the previous sections. The method was developed by Henshaw [47], and Henshaw, Kreiss, and Reyna [48] for solving the formulation of the incompressible Navier-Stokes equations on structured overlapping grids. The governing equations will be expressed in Cartesian coordinates for simplicity. In order to transform the governing equations from physical space to computational space, the Cartesian derivatives in the governing equations need to be replaced by their transformed equivalent in computational space. Recalling equation (3.9), the initial-boundary-value problem (IBVP) for the Navier-Stokes equation is

$$
\begin{gathered}
\frac{\vec{u}}{\partial t}+\vec{u} \cdot \nabla \vec{u}=\frac{-\nabla p}{\rho}+v \nabla^{2} \vec{u} \text { for } x \in D, t>0 \\
\nabla \cdot \vec{u}=0 \text { for } x \in D, t>0
\end{gathered}
$$

with the initial conditions and boundary conditions

$$
\begin{gathered}
\vec{u}(\vec{x}, t)=\vec{u}_{0}(\vec{x}) \text { for } x \in D, t_{0}=0 \\
B(\vec{u}, p)=g \text { for } x \in \partial D, t \geq 0
\end{gathered}
$$

In this IBVP, the vector contains the Cartesian coordinates $(\mathrm{x}, \mathrm{y}, \mathrm{z})$ in physical space $P$, and $D$ is a bounded domain in (where $\mathrm{N}=1,2,3$, ), is the boundary of the domain $D, t$ is the physical time, vector contains the Cartesian velocities in physical 
space, $p$ is the pressure, $\rho$ is the fluid density, $\nu$ is the fluid kinematic viscosity, $B$ is a boundary operator, $g$ is the boundary data, and is the initial data. This system of equations is called the velocity-divergence formulation of the Navier-Stokes equations. Applying the divergence operator to the Navier-Stokes equations and taking consideration of the continuity equation, a governing equation for the pressure field can be derived. Consequently, the following alternative system of equations called the velocity-pressure formulation can be created $[46,6]$

$$
\begin{gathered}
\frac{\vec{u}}{\partial t}+\vec{u} \cdot \nabla \vec{u}=\frac{-\nabla p}{\rho}+v \nabla^{2} \vec{u} \text { for } x \in D, t>0 \\
\frac{\nabla^{2} p}{\rho}+\nabla u \cdot \overrightarrow{u_{x}}+\nabla v \cdot \overrightarrow{u_{y}}+\nabla w \cdot \overrightarrow{u_{z}}=0 \text { for } x \in D, t>0
\end{gathered}
$$

The corresponding boundary and initial conditions became

$$
\begin{gathered}
\nabla \cdot \vec{u}=0 \text { for } x \in \partial D, t>0 \\
B(\vec{u}, p)=g \text { for } x \in \partial D, t>0 \\
\vec{u}\left(x, t_{0}\right)=\overrightarrow{u_{0}}(x) \text { for } x \in \partial D, t=0
\end{gathered}
$$

Using equations (3.21) and (3.22), an approximate numerical solution is usually sought after in a given domain $\mathrm{D}$ with the prescribed boundary and initial conditions, equations (3.23)-(3.25). Equation (3.22) implies that the pressure can be calculated provided the velocity field is known, this is called the Poisson-pressure equation (PPE). The PPE is derived by taking the divergence of the momentum equation (3.17) and using the divergence-free constraint of Eqn. 3.23. The constraint is replaced by the elliptic equation for the pressure. In order for this set of equations to be well defined, an extra boundary condition is required. The extra condition of 
$\nabla \cdot \vec{u}=0$ for $x \in \partial D$ is added. This ensures the system of equations are equivalent to the original formulation (equations (3.17)-(3.20)) [48]. However, the lack of a proper explicit boundary condition for the PPE causes trouble when implementing the numerical model based on the velocity-pressure formulation. To compensate, the use of the normal component,(3.21), of the momentum equation (3.21), allows us to obtain

$$
\frac{\partial p}{\partial \hat{n}}=\hat{n} \cdot\left(-\overrightarrow{u_{t}}-(\vec{u} \cdot \nabla) \vec{u}+\nu \nabla^{2} \vec{u}\right) \rho \text { for } x \in \partial D, t=0
$$

A divergence term is added in the form of $\alpha_{d c} \nabla \cdot \vec{u}$ to the PPE to suppress the spurious divergence, so that equation (3.22) becomes

$$
\frac{\nabla^{2} p}{\rho}+\nabla u \cdot \overrightarrow{u_{x}}+\nabla v \cdot \overrightarrow{u_{y}}+\nabla w \cdot \overrightarrow{u_{z}}-\alpha_{d c} \nabla \cdot \vec{u}=0 \text { for } x \in \partial D, t>0
$$

This extra divergence term can be interpreted as a divergence sink or an artificial damping term, helping to keep the divergence small. The divergence is primarily caused by truncation errors and calculation errors between the interpolation of information between computational domains. The $\alpha_{d c}$ coefficient is usually selected by the user and is discussed in Henshaw [48], Henshaw, Kreiss, and Reyna [48]. If $\alpha_{d c}$ is properly chosen, it will prevent spurious divergence, and have minimum impact on the calculation accuracy. If $\alpha_{d c}$ it is too small, the system may become unstable, if too large; the system may be over dampened.

Equation (3.21) is discretized into computational space using second-order centered finite-difference approximations. The resulting system of equations can be expressed in a system of ordinary differential equations of the form

$$
\frac{d \vec{u}}{d t}=F(\vec{u}, p, t)
$$

The velocity field can be solved with a given pressure field which is either by solving equation (3.27) or from an initial guess. In order to keep the pressure equation 


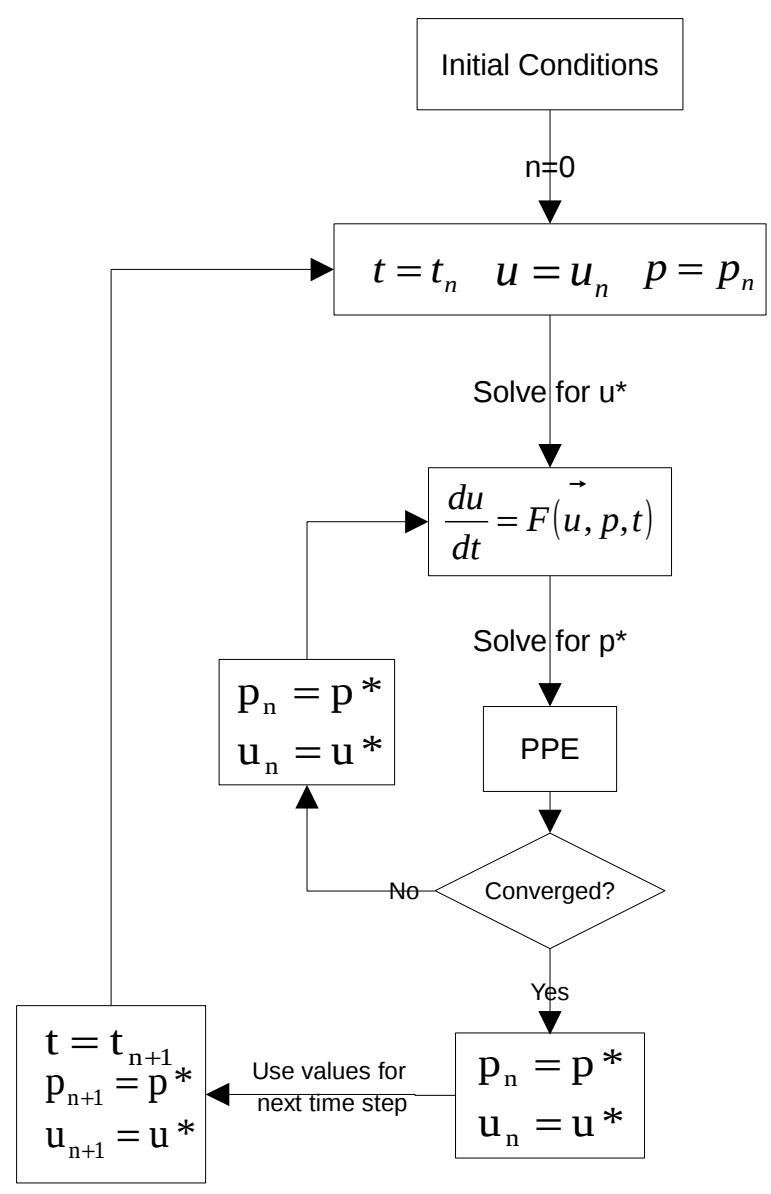

Figure 6: Process diagram of split-step scheme of the Navier-Stokes equation

decoupled from the solution of the velocity components, a time stepping scheme must be chosen for the velocity components that involves the pressure from the previous time step. This is called a split-step scheme. Then the equations are solved using a semi-implicit multistep method that uses a Crank-Nicolson scheme for the viscous terms and second-order Adams-Bashforth predictor-corrector for the convective terms and pressure. [6]

The entire process is illustrated in figure 6 , where an initial condition of $U_{0}$ and $p_{0}$ are inputted at time zero. $U^{*}$, an intermediate velocity value, is solved using equation (3.28), which then is inputted into the PPE, equation (3.27). An intermediate pressure, $p^{*}$, is solved, and $U^{*}$ and $p^{*}$ checked for convergence, if not, both are inputted back into equation (3.27). Once converged, $p^{*}$ and $U^{*}$ become the solution 
to the pressure and velocity at that time step, and then are used to initialize the next time step.

\section{Boundary Conditions}

Other boundary conditions can be imposed on the incompressible NavierStokes equations than previously discussed. Using the appropriate conditions is paramount in solving any governing equations. The types of boundary conditions are dependent on the type of flow, and geometry for a selected problem. Once these two criteria have been determined, it is possible to numerically solve the velocitypressure equation using the Overture framework coupled with the PETSc ${ }^{1}$ library. Inside the Overture package, Cgins is used to solve the velocity-pressure equation discretized across an overlapping grid. Cgins offers easy implementation of boundary conditions, and offers the following types [48].

$$
\begin{aligned}
& \text { No-Slip }=\left\{\begin{array}{cl}
\overrightarrow{\mathbf{u}}=g & \text { Velocity specified } \\
\nabla \cdot \overrightarrow{\mathbf{u}}=0 & \text { Zero divergence }
\end{array}\right. \\
& \text { Slip wall }=\left\{\begin{array}{cl}
\hat{\mathbf{n}} \cdot \overrightarrow{\mathbf{u}}=g & \text { Normal velocity specified } \\
\partial_{\hat{n}}(\hat{t} \cdot \overrightarrow{\mathbf{u}})=0 & \text { Normal derivative of tangential } \\
\nabla \cdot \overrightarrow{\mathbf{u}}=0 & \text { velocity is zero } \\
& \text { Zero divergence }
\end{array}\right. \\
& \begin{aligned}
\text { Inflow with velocity } \\
\text { given }
\end{aligned}=\left\{\begin{array}{cl}
\overrightarrow{\mathbf{u}}=g & \text { Velocity specified } \\
\partial_{\hat{n}} p=0 & \text { Normal derivative of the pressure } \\
\text { zero }
\end{array}\right. \\
& \text { Outflow }= \begin{cases}\text { extraplotate } \quad \overrightarrow{\mathbf{u}} & \text { Velocity specified } \\
\alpha p+\beta \partial_{\hat{n}} p=g & \text { Mixed derivative of } p \text { given }\end{cases} \\
& \begin{aligned}
\text { Dirichlet boundary } \\
\text { condition }
\end{aligned}= \begin{cases}\overrightarrow{\mathbf{u}}=g & \text { Velocity specified } \\
p=P & \text { Pressure given }\end{cases} \\
& \text { Symmetry }=\left\{\begin{array}{cl}
\hat{\mathbf{n}} \cdot \overrightarrow{\mathbf{u}}: \text { odd }, \quad \hat{\mathbf{t}} \cdot \overrightarrow{\mathbf{u}}: \text { even } & \text { Vector symmetry } \\
\partial_{\hat{n}} p=0 & \text { Normal derivative of pressure zero }
\end{array}\right.
\end{aligned}
$$

\footnotetext{
${ }^{1}$ http://www.mcs.anl.gov/petsc/
} 


\section{Rigid Body Motion}

For moving overlapping grids, the governing equations are reformulated in the reference frame of the moving grid. Equations 3.21 and 3.17 are expressed in the moving reference grid of the component grid as follows:

$$
\begin{aligned}
& \frac{\vec{u}}{\partial t}+[(\vec{u}-\dot{G}) \cdot \nabla] \vec{u}=\frac{-\nabla p}{\rho}+v \nabla^{2} \vec{u} \text { for } x \in D, t>0 \\
& \frac{\partial p}{\partial \hat{n}}=\hat{n} \cdot\left(-\overrightarrow{u_{t}}-(\vec{u} \cdot \nabla) \vec{u}+\nu \nabla^{2} \vec{u}\right) \rho \text { for } x \in \partial D, t>0
\end{aligned}
$$

where $\dot{G}$ is the rate of change of the position of the given set of grid points $x_{\wp}^{g}$ in the physical space $\wp$. For the component grids that are moving, the steady boundary conditions no longer are valid, and must must be expressed in the moving reference frame. For a moving body with a corresponding moving no-slip wall, the new constraint corresponds to the velocity of the wall where

$$
\vec{u}\left(\left.x_{\wp}^{g}\right|_{\text {wall }}, t\right)=\dot{G}\left(\left.x_{\wp}^{g}\right|_{\text {wall }}, t\right) \text {, where }\left.x_{\wp}^{g}\right|_{\text {wall }} \in \partial D_{\text {wall }}(t)
$$

On a moving no-slip wall the boundary condition for the pressure equation is obtained by dotting the normal $\hat{n}$ into the momentum equation.

$$
\left.\frac{\partial p}{\partial \hat{n}}\right|_{\partial D_{\text {wall }}}=\left.\hat{n}\right|_{\partial D_{\text {wall }}} \cdot\left(-\ddot{G}+\nu \nabla^{2} \vec{u}\right) p
$$

\section{E. Q-criterion}

To properly conduct this study, a numerical method to identify vortices must be implemented in order to decipher actual vortices from shear vorticity. If no detection scheme is used, definition of a vortex from streamlines or vorticity plots is ambiguous at best. Over a dozen individual detection schemes exist today for vortex identification. For this study the Q-criterion, originally developed by Hunt et al. [49], was selected. The Q-criterion is a Galilean-invariant vortex criterion that is based off 
the velocity gradient tensor, $\nabla u$, of the flow field where $u$ is the velocity vector. The Q-criterion is a straight forward calculation shown in Eqn. 3.33 where $\Omega$ and $S$ are the anti-symmetric and symmetric portion of $\nabla u$ respectively. The anti-symmetric term $\Omega$ is also known as the vorticity tensor, thus the symmetric $S$ is known as the strain rate tensor.

$$
\begin{gathered}
Q=\frac{1}{2}\left(|\Omega|^{2}-|S|^{2}\right)>0 \\
\Omega=\frac{1}{2}\left[\nabla u-(\nabla u)^{T}\right] \\
S=\frac{1}{2}\left[\nabla u+(\nabla u)^{T}\right]
\end{gathered}
$$

A vortex is positively identified when $\mathrm{Q}$ is greater than zero. This occurs when the vorticity tensor, $|\Omega|$, is greater than the strain rate tensor, $|S|$. In addition to the $Q>0$ requirement, the pressure at the center of the vortex must be less than ambient

pressure. The Q-Criterion was implemented into the Overture framework, to later provide a method to filter vortex related vorticity from shear vorticity. An example of the Q-criterion is shown in figure 7. While the vortex in the example shown is easy to identify, the Q-criterion gives an analytical definition of the boundary of the vortex.

\section{F. Overlapping Grids}

\section{Types of Grid Generation}

In the last section, the governing equations for low speed incompressible flows were discussed. Developing the equations is only part of the solution for solving flow fields in complex domains. A grid or mesh must be generated to allow for the governing PDEs to be spatially discretized. Grid generation can be described as 


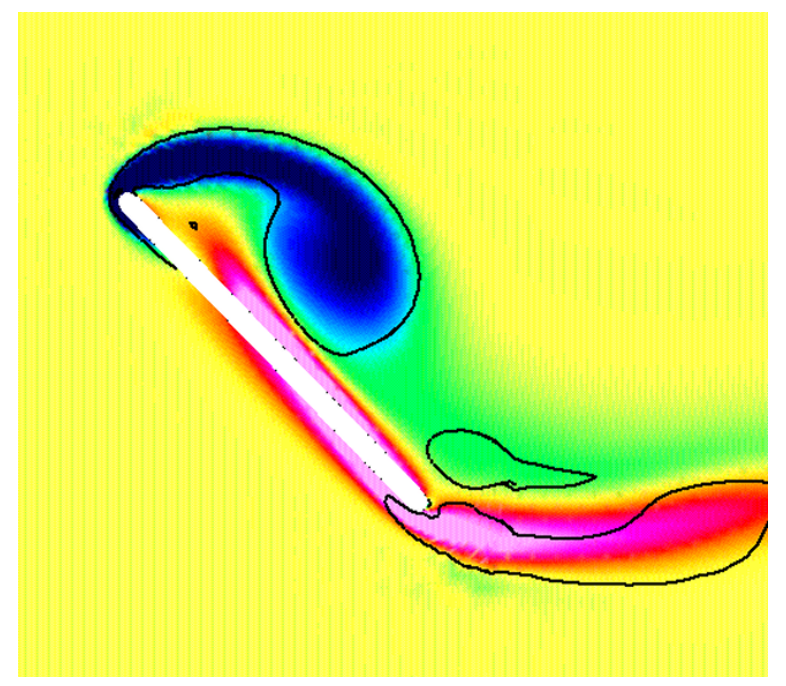

Figure 7: LEV vorticity contour at mid-span with the Q-criterion (black line) defining the boundaries of identified vortices at $45^{\circ}$.

breaking a continuous domain into smaller domains. There are three main types of grids: body-fitting structured grids, unstructured grids, and Cartesian grids [50].

Body-fitting structured grids are constructed of repeating rectangular blocks, thus where it derives its name. In structured grids, the domain is rectangular in shape where the interior grid points are distributed along the grid lines. This allows for easy identification of each grid point in reference to the grid lines. Figure 8 illustrates a simple structured grid fitted to an annulus inside a square domain. Structured grids also provide a major advantage over other types of grid generation. They allow for a high degree of user control. The user has total control how the mesh is generated and positioned. This enables the user to place control schemes that concentrate points in regions of complex flow interactions, such as boundary layers. Because the grid is user controlled, this allows for the grid to be developed in a fashion that maximizes the computational efficiency of the grid, allowing for faster, and more accurate computed PDE solutions.

The main disadvantage to structured grids occurs when a multi-block method has to be employed. When complex geometries need to be blocked to non-trivial boundaries, the user can break the domain into several sections. Each section has 


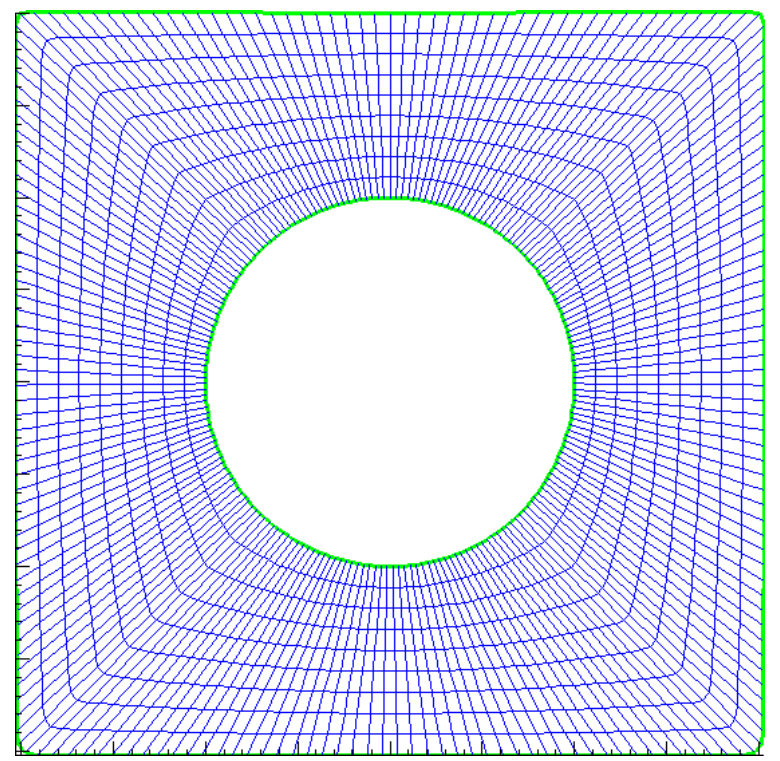

Figure 8: A structured grid meshed between an annulus and square.

independently generated structured grids inside of them, and then a grid is generated between the sections to connect them. Figure 9 shows the same annulus and square domain as in figure 8 but created with multiple structured grids. One grid generated around the annulus, to allow for a more uniform grid around it, while four other structured grids are meshed to fit the square. While this gives the user more control in generating grids for complex geometries, connecting the grids can be difficult and tedious. Because of this problem, structured grid generation sometimes requires a high level of skill from the user, and sometimes takes weeks to create an optimized grid.

Unstructured grids resolve the some of the problems associated to structured grids. Instead of using rectangular elements, unstructured grids use triangular elements. A generated unstructured grid has no recognizable pattern as a structured grid has, and this is why it is called unstructured. One advantage to unstructured grids is that elements are much easier to position to fit the requirements of the domain. Another is the process of grid generation can be automated to a fairly large degree, requiring very little user input. The user does not have to worry about laying out 


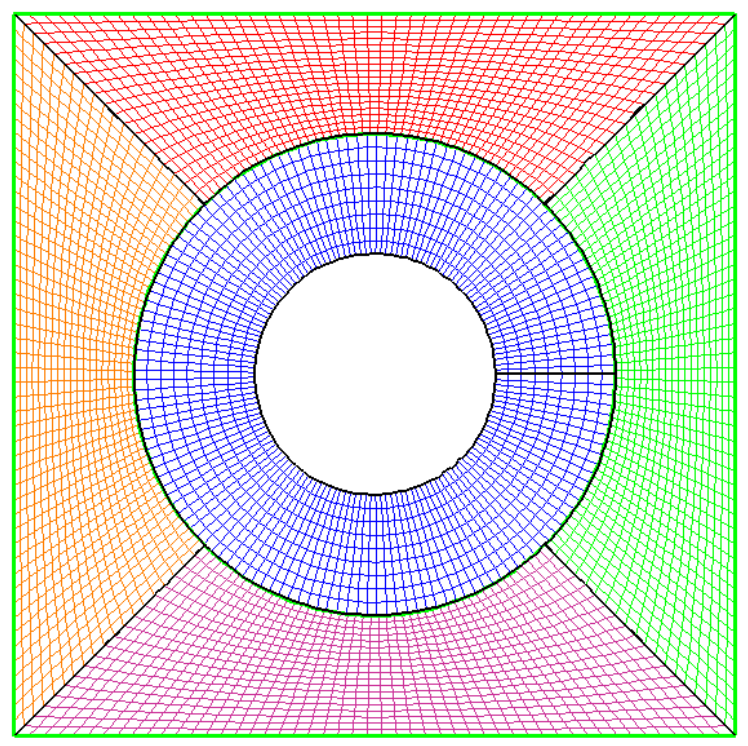

Figure 9: A multiblocked structured grid.

properly generated structured sections, and then connecting them. This allows inexperienced users to quickly generate grids in a matter of minutes or hours as opposed to days or weeks. Figure 10 shows an example of the annulus and square meshed with an unstructured grid.

The major disadvantage to unstructured grids is the lack of controllability. Usually the user has very limited control in how the grid is generated and positioned. Also the triangular elements do not stretch well, the grids usually appear uniform. Unlike structured grids, unstructured grids cannot be locally refined to meet the needs of the user. Thus often times, regionally grid densities have to be increased as opposed to local increases. The last drawback is that unstructured grids take significantly more time and memory to solve. This is because unstructured grids do not have an easy grid point reference system like the structured grids. Both methods can be referred to as body-fitted grid generation. This means the blocks near the geometries surface must conform to that surface. As a result the surface mesh can conflict with itself, and the requirements of the user and the flow field. To resolve this issue, a resurgence of interest in Cartesian grids has come about in the last decade. 


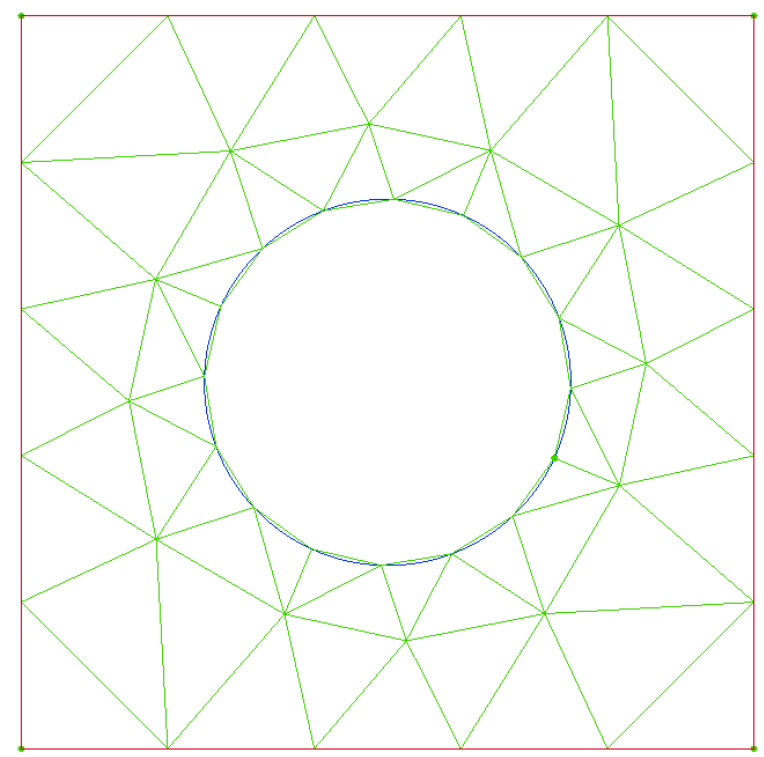

Figure 10: Unstructured annulus and square mesh [4]

The Cartesian method abandons the use of body-fitted grids all together. Instead it uses a Cartesian background grid. The geometry is cut out of the grid leaving a set of irregularly shaped cells on the boundary of the geometry. Cartesian grids use a second method called adaptive mesh refinement (AMR) [51]. Without this automatic refinement, the Cartesian grids would lack the ability to efficiently resolve fluid and geometry features. This works by actively adding more uniform grid points in areas of where the flow field is not converging until the flow field is resolve to a satisfactory degree. This is a major advantage to Cartesian grid generation, because the grid generation is fully automated. Since there are no body-fitted grids, the grid quality and refinement can be fully automatically. Although because of the geometry is not smooth but, almost stair case like ([6]), this puts a greater burden on the flow solver. Figure 11 illustrates how the annulus and square example would look. The annulus loses its smooth edge, however a finer Cartesian grid could reduce the staircase effect. However, highly efficient and accurate flow solvers can be implemented easier using this technique resulting in better solutions. The single drawback of Cartesian grids is that their use is currently restricted to inviscid or low Reynolds number flows [51]. 


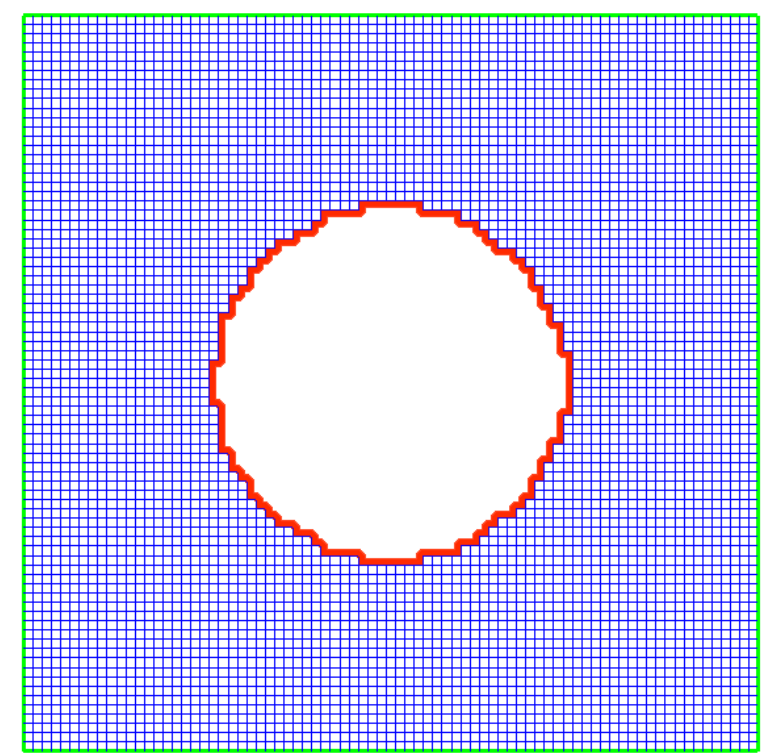

Figure 11: Cartesian grid of annulus and square.

These three approaches discussed are the most basic forms of grid generation. The next section, a newer form of grid generation methodology based on structured grids that resolve many of its inheriting problems will be described.

\section{G. Overlapping Structured Grids}

Overlapping structured grids, also known as composite grids or Chimera grids, provide a more flexible method of grid generation, yet still provide efficient spatial discretization for numerically solving the governing PDEs. The overlapping grid method consists of generating a set of body-fitted structured grids that completely cover the physical domain that is being modeled and overlap where they meet. Like previous figures,figure 11 illustrates how overlapping grids usually function. A structured grid is body-fitted around the annulus, and then overlaid onto of a uniform background grid. The governing PDEs are solved separately on each grid and then connected to each other through interpolation in the overlapping areas. With the ability to define each of the components individually, the grids around them can also be generated individually also. 


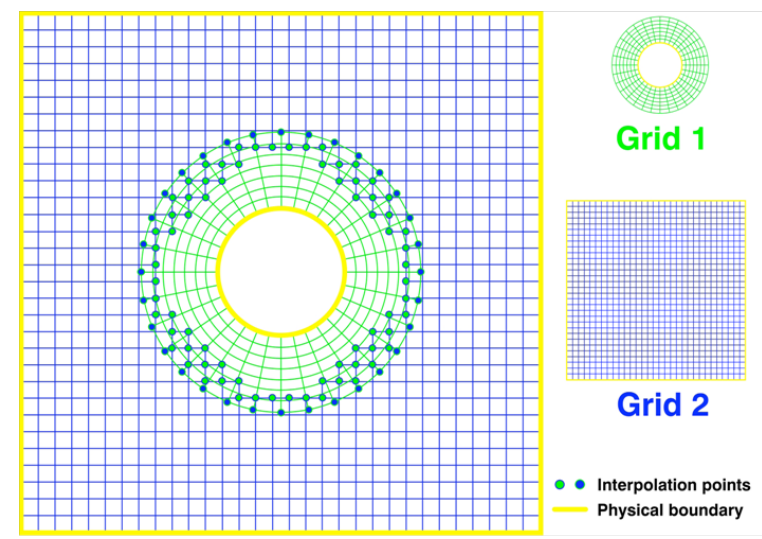

Figure 12: Example of Overlapping Structured Grid system [5, 6]

Normally body-fitted grids are used near the geometry of the model, while one or more Cartesian grids are used in the background to handle the rest of the domain. Overlapping grids were originally developed to address complex geometry, multiple body motion, and to resolve fine-scale flow features through the use of AMR. The generated grids can be easily altered and reproduced nearly instantaneously. This allows for multiple iterations of the same grid to be simulated with different parameters with ease [52]. This is done by only modifying the grid of interest and reoverlapping the grids, as opposed to modifying the entire domain grid structure. Through the use of structured grids coupled with Cartesian grids, a method has been developed that is optimized the spatial discretizations has led to a highly efficient method in computer time and memory expense. [52] While the overlapping grids method has been in use for some time now, it hasn'tt been of much interest until recently. It was originally described by Volvov in 1966 [53]. The method was further developed and promoted by Starius in 1977 [54]. Even the method is fairly old; it was not in introduced to the CFD community until the early 1990s. Steger et al. [55] and Benek et al. [56]; and it has been further developed by Meakin and Suhs [57], Chesshire and Henshaw [58] and Noack et al. [59]. This approach is now recognized as an attractive method for solving problems with complex geometries and moving bodies. [6] As with all methods, overlapping grids have a few disadvantages. The 
algorithms to generate the overlapping grids can be far more complex. Mistakes can be easily made in setting grid boundary conditions or overlapping priorities. This makes grid generation an iterative process. If proper planning in the generation process is not done, the overlapping grids can lead to poor interpolation results between grids, leading to increased computational time and poor solution convergence. However, if good generation practices are established, many problems can be avoided all together, and grids can be generated fairly quickly. In addition to added complexity, the flow solvers used must be able to handle interpolation across multiple overlapping grids. The data structures required to connect overlapping grids can cause problems if the flow solver adequately programmed correctly. However, even though the process can be more complex compared to purely structured, or unstructured grid domains, the gain in computational efficiency and the ease of altering established grids makes the method worthwhile. In the next section, it will be discussed how Ogen [7], a tool in the Overture ${ }^{2}$ package, determines how different grids will interpolate with each other, by eliminating grid points in regions of excess overlap.

\section{H. Overlapping Grid Assembly}

In this section, it will be discussed how Ogen, the grid generation tool inside of Overture, assembles overlapping grids. The assembly of overlapping grids consists of three major parts. The first step is the definition of the geometer and grid component generation by the user. The second step consists of grid generation tool detecting all grid points to be removed. The final step, is locating all grid points to be used for interpolation on the edge of the removed points. Only a brief discussion will be given on how the algorithm works, for a more detailed discussion, the process is well documented in Henshaws paper [7]. To properly describe the major steps and its corresponding sub steps, first a set of component grids and boundary conditions must be defined. Figure 13 illustrates the two component grids that will be used in

\footnotetext{
${ }^{2}$ http://www.overtureframework.org/
} 


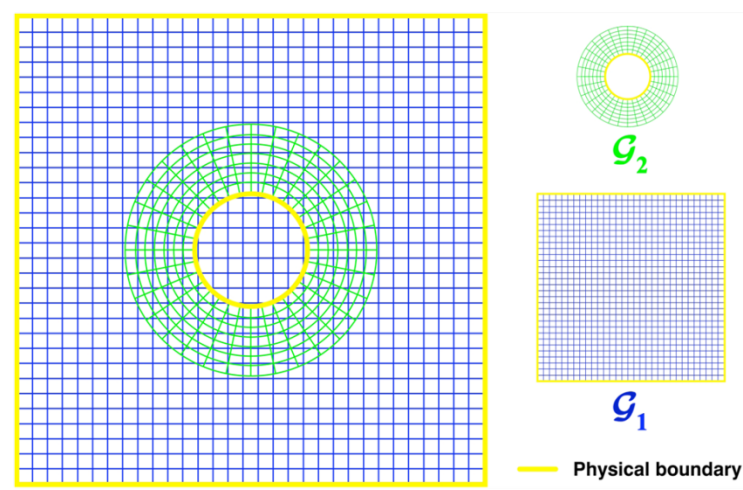

Figure 13: Component grids a Cartesian grid, G1, and a body-fitted structured grid around an annulus, G2. [6, 7]

this example. The annulus, G2, is an annulus with a structured body-fitted grid, and G1, is a Cartesian background grid.

The next stop begins with the software marking the hole boundaries and removing exterior grid points. This process itself has two-substeps. Firstly, each physical boundary, points are found that lay inside, or outside of other physical boundaries. Therefore, the algorithm will see grid points from G1 are laying inside of G2, and marked as unused points. After this substep, the unused points will be bounded by a boundary of interpolation points as illustrated in figure 14. Once this is down, all unused points will be removed. This completes the second step, and the algorithm has created what is known as a Chimera hole [7].

The final step finds and classifies all valid interpolation points. Here, the points on the physical boundaries and interpolation boundaries are collected into a list of interpolation points. Then points are labeled using improper interpolation. A point is said to be improper if it lies inside of another grid. Since all points lie within the domain, they all must interpolate from some other grid. From these improper points, proper points must be then classified. Proper points are points that lie along the lines of the two grids being overlapped. These lines will be used for implicit or explicit interpolation for the flow solvers [7]. Once this done, an overlapping grid system has been created. Figure 15 shows what the newly formed system looks like. 
Interpolation points $\square$ Unused points Physical boundaries

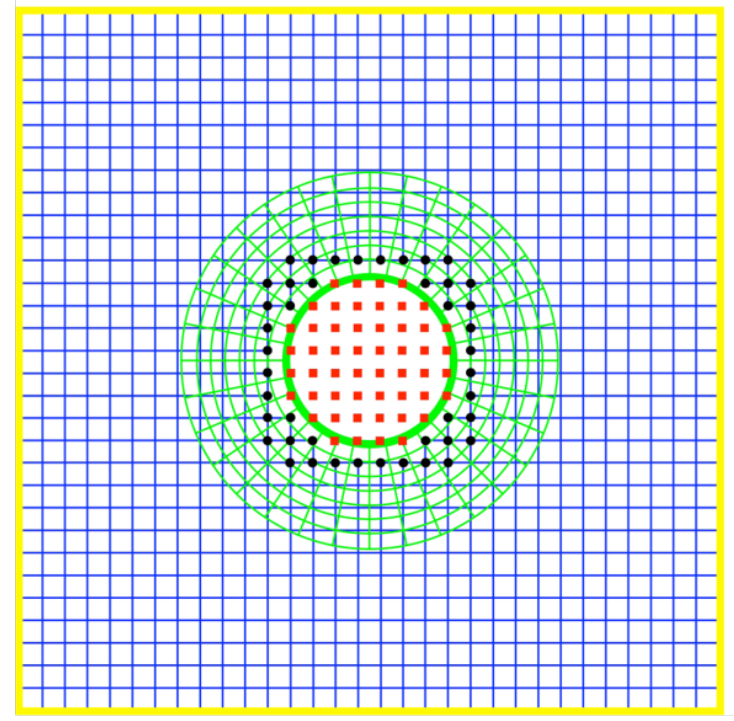

Figure 14: Identifying unused, and interpolation points $[6,7]$

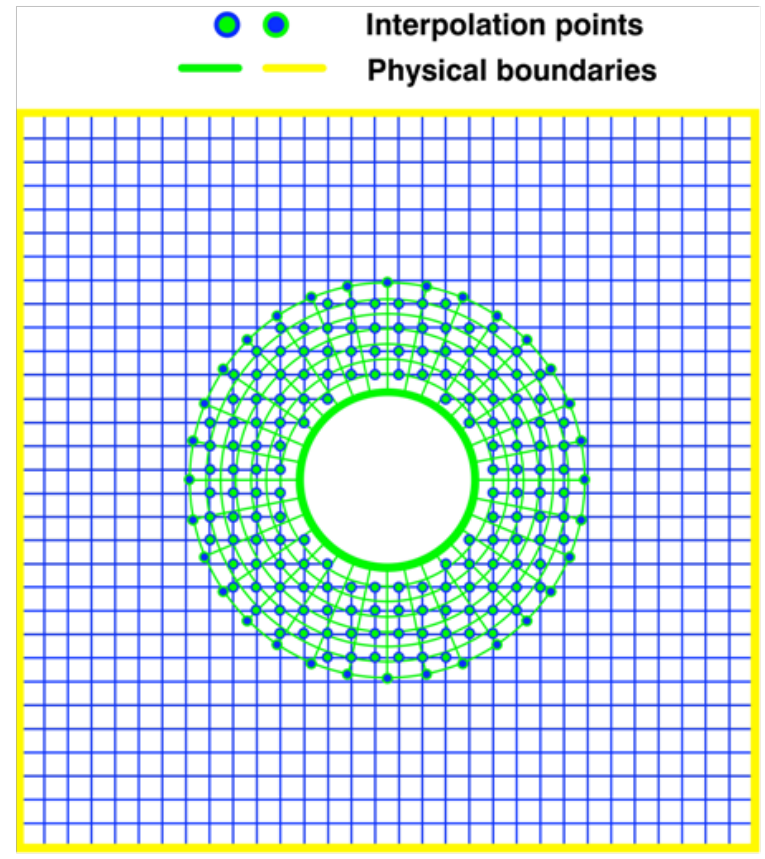

Figure 15: Completed overlapping grid system $[6,7]$ 


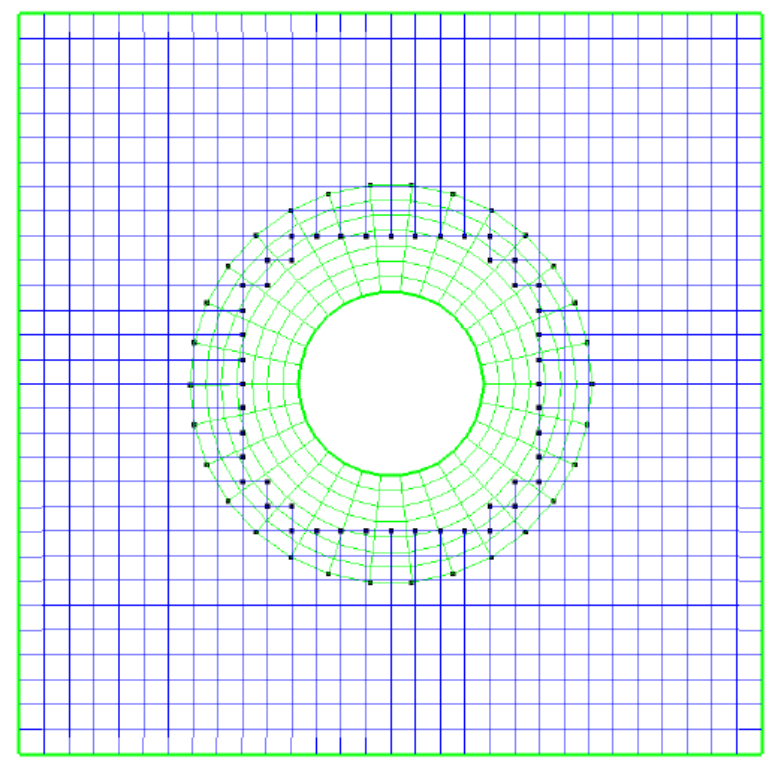

Figure 16: Trimmed overlapping grid $[6,7]$

An additional set can be performed at this stage called trimming. Basically, it reduces the amount of overlapped between the two grids. This is done by removing any interpolation point deemed not needed. The amount of overlapped removed is usually specified by the user or a minimum overlap requirement preset in the algorithm. The final trimmed overlapping grid can be seen in figure 16.

This completes the major three steps in constructing an overlapping grid. A forth step is also usually completed which checks the consistency of the system to see if it satisfies all requirements. If not, all points that fail to satisfy the requirements will be marked, and outputted as a graphic for troubleshooting. 


\section{CHAPTER IV}

\section{CODE VALIDATION}

Before beginning the numerical study, the Overture solver was first validated against several low Reynolds number cases. First a shedding cylinder case was examined to investigate the solver's accuracy in predicting bluff body shedding flows. Next, the solver's ability to handle moving bodies was also examined by reproducing a numerically studied pure plunging wing case. Finally, the a single pitch-up wing case was compared against experimental results.

For the three tested cases, lift and drag coefficients are compared with published data. Both lift and drag coefficients are calculated as follows:

$$
\begin{aligned}
& C_{L}=\frac{F_{L}}{2 \rho A V^{2}} \\
& C_{D}=\frac{F_{D}}{2 \rho A V^{2}}
\end{aligned}
$$

Where $F$ is the lift or drag force, $A$ is the area (assumed to be one for 2D) and $V$ is the free stream velocity.

\section{A. Shedding Cylinder}

2D shedding cylinder cases are widely used to validate time-accurate solvers, and thus a plethora of data exists for comparison. Here a cylinder case was reproduced that exists extensively in the literature $[60,61,6,62,63,64,65]$ with the following

domain schematic in figure 17. This particular case has been conducted at a variety 


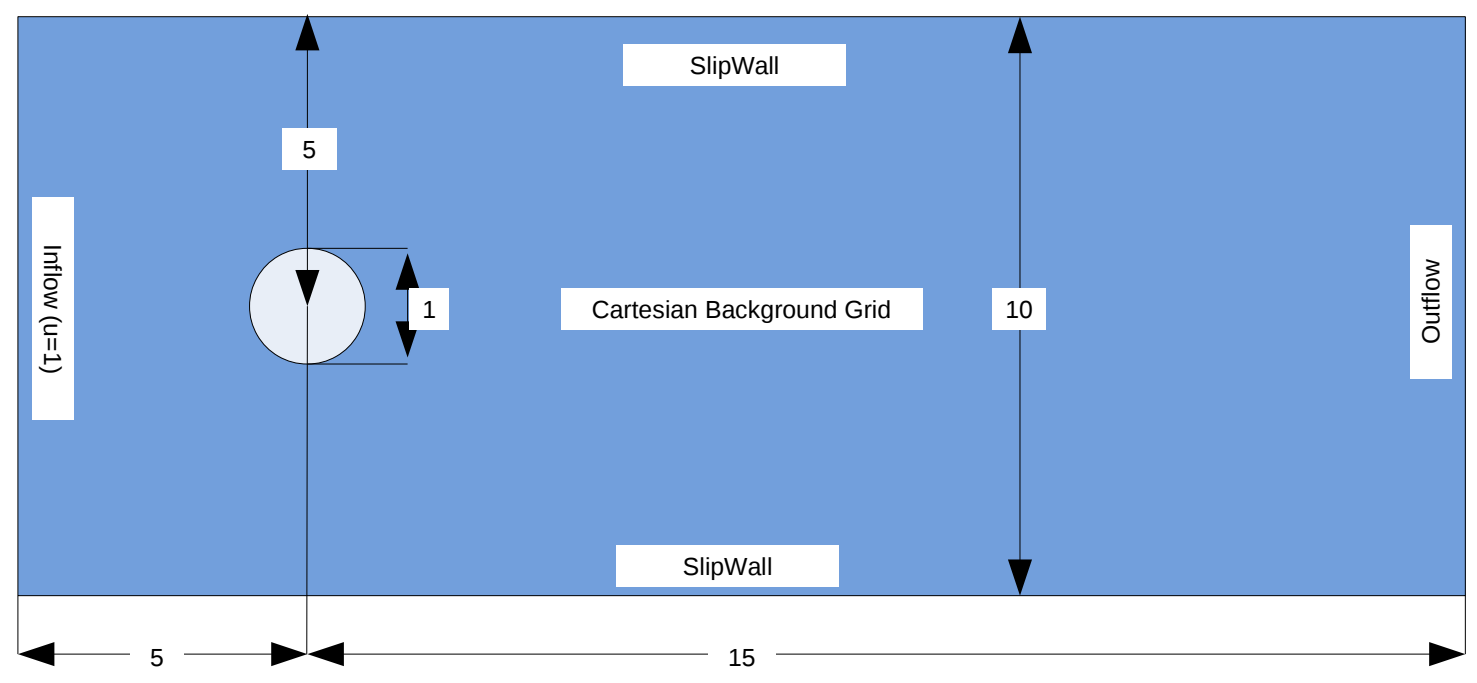

Figure 17: Overlapping grid layout used for code validation

of Reynolds numbers due to the fact that $2 \mathrm{D}$ cylinders do not begin to shed until the Reynolds number is above 50 .

For this validation case, the Reynolds number was based on the diameter of the cylinder, the kinematic viscosity and the inflow velocity. By varying the kinematic viscosity, the flow solver was tested at Reynolds numbers of 20, 40, 100, and 200 . Table 1 lists the numerical results of the coefficient of drag compared to published data. At Reynolds numbers greater than 50, the flow becomes unsteady and the \pm denotes the amplitude of the drag oscillations. It was found that the flow solver produced results within the published range confirming the validity of the flow solver.

\section{B. 2D Pure Plunge}

To test Overture's capability in handling moving body simulations, a case reported by Visbal[66] was studied. Visbal studied the aerodynamics of a SD7003 airfoil subjected to a pure sinusoidal plunge motion at a Reynolds number of 1000 . His numerical simulations were performed using a $6^{\text {th }}$ order Navier-Stokes solver, FDL3DI[67], and a 3D domain with periodic spanwise boundary conditions. This case was selected due to the large LEV that sheds from the airfoil during the plunge 


\begin{tabular}{|l|c|c|c|c|}
\hline & \multicolumn{4}{|c|}{$C_{D}$ for Reynolds Number } \\
\hline Reference & $\mathbf{2 0}$ & $\mathbf{4 0}$ & $\mathbf{1 0 0}$ & $\mathbf{2 0 0}$ \\
\hline Calhoun and Wang [60] & 2.19 & 1.62 & $1.35 \pm 0.014$ & $1.17 \pm 0.058$ \\
\hline Choi et al. [61] & - & - & $1.34 \pm 0.011$ & $1.36 \pm 0.048$ \\
\hline Guerroro [6] & 2.2013 & 1.6208 & $1.39 \pm 0.012$ & $1.41 \pm 0.098$ \\
\hline Kim and Choi [62] & - & 1.501 & 1.336 & - \\
\hline Posdziech and Grundmann [63] & 2.07 & 1.54 & 1.35 & 1.35 \\
\hline Russel and Wang [64] & 2.13 & 1.6 & $1.38 \pm 0.007$ & $1.29 \pm 0.022$ \\
\hline Tritton [65] & 2.22 & 1.48 & - & - \\
\hline Numerical Results & 2.23 & 1.59 & $1.42 \pm 0.007$ & $1.37 \pm 0.030$ \\
\hline
\end{tabular}

TABLE 1

Comparison of CD with published results at varying Reynolds numbers

motion, similar to a dynamic stall process. To compare Overture's capability, a 2D overlapping mesh to check the solver's ability to predict the lift force. The SD7003 was defined by a structured body-fitted mesh with 180 points in the circumferential direction. The distance of the first grid point to the airfoil is $3.36 \times 10^{-3}$. The surrounding domain was 10 chords away from all sides of the airfoil and was found to be large enough to have no effect on lift and drag calculations. Figure 18 compares our simulated lift coefficient and Visbal's[66]. (Drag was not reported in his paper). There is little deviation between the two simulations, and demonstrates that the code is able to predict the lift force in this Reynolds number regime due to shedding vortices. 


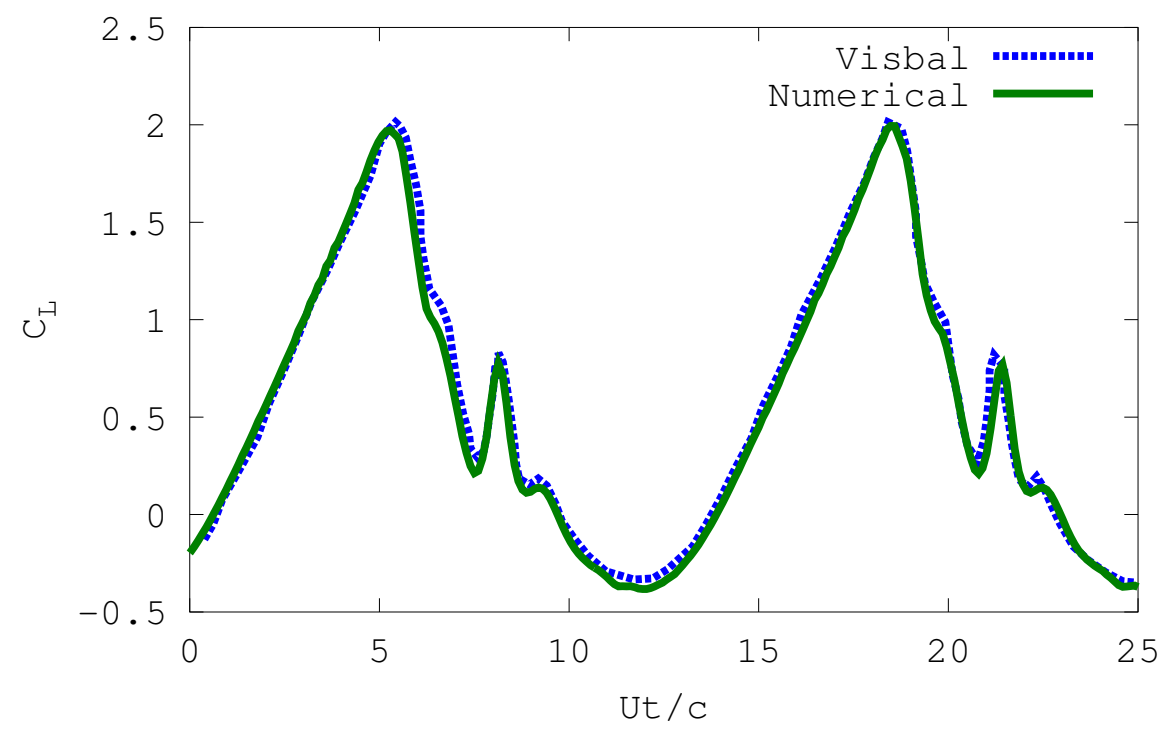

Figure 18: Comparison of lift coefficients of a SD7003 airfoil undergoing a sinusoidal plunge motion.

\section{2D Single Pitch}

Finally, the solver was validated against a higher Reynolds number case of 20,000 where a wing was pitched up about the leading edge. This case has been experimentally studied by Granlund et al.[8] and will be referenced later in the text. The experiment was conducted at a Reynolds number of 20,000 using wall-to-wall flat plate wing. The wing's thickness was equal to $5 \%$ of its cord length. The pitching kinematics follows Eqn. (2.3), and pitches about the leading edge. Our simulation used a 2D structured body-fitted mesh which had 350 points on the airfoil with an initial cell height of $1.1 \times 10^{-3}$. The domain was 10 chords away from all sides of the airfoil. Figure 19 compares the simulated and measured lift and drag coefficients. The simulated drag coefficient is slightly lower than the measured one but overall the agreement is good. 

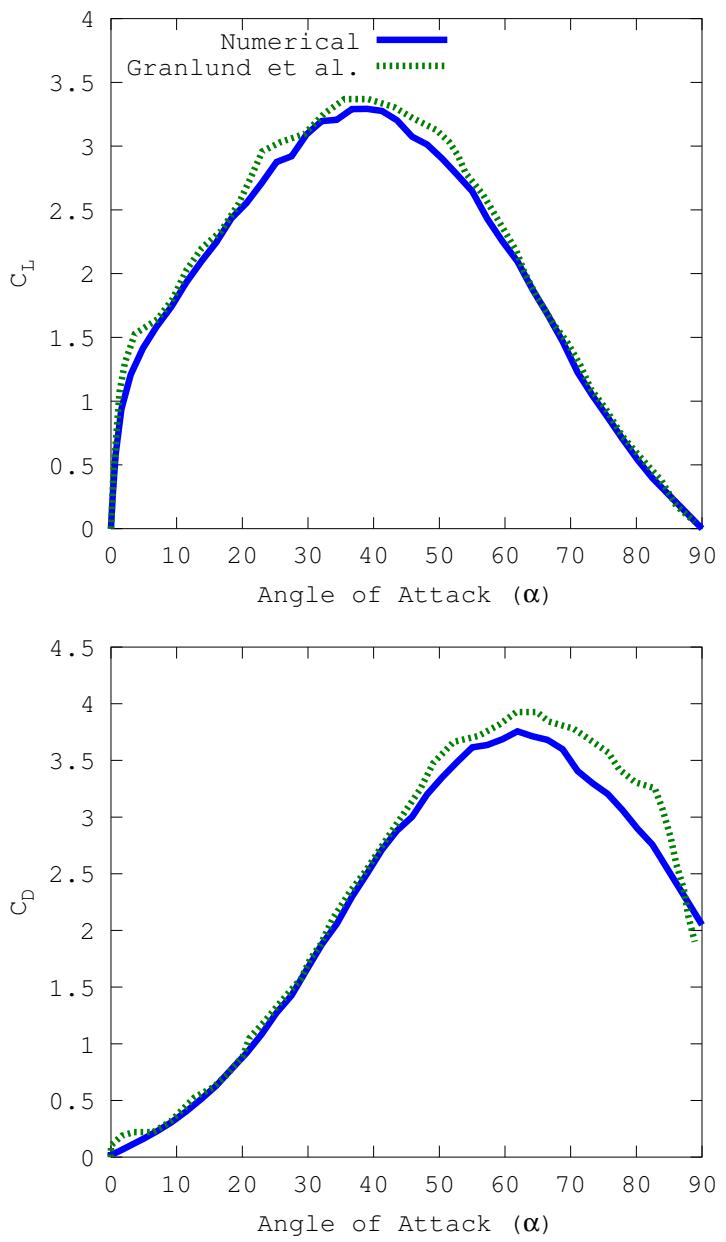

Figure 19: 2D lift and drag coefficients of a flat plate pitching up around the leading edge at $K=0.2$ and $R e=20,000$. 


\section{CHAPTER V}

\section{COMPUTATIONAL SETUP}

\section{A. Summary}

Due to computational limitations of the solver, only serial processing is available. A grid sensitivity analysis was conducted to maximize the accuracy of the simulated results and to minimize the computational cost. Both 2D and 3D grids had their grid densities varied to understand the impact of the spatial resolution on the lift and drag forces. In this section, the validation of the 3D grid is the primary focus as the $2 \mathrm{D}$ grids have been validated in another study using the same solver[68].

Due to the limitations of Overture, all cases were ran in serial on a 2.2 gigahertz processor. The largest case $(A R=4, K=0.1)$ required approximately 680 hours of computation time and 2 gigabytes of RAM. Output data sizes varied with output frequency, but was approximately 15 megabytes per file. Each simulation on average required 1200 megabytes of harddrive space.

\section{B. Grid Generation}

In both the 2D and 3D studies, the wing profile tested was a 5\% thick flat plate with rounded leading and trailing edges shown in figure 20. For the 3D study, the wing chord was held constant as the span of the wing was varied to achieve different aspect ratios $(A R=1,2,4)$ defined by Eqn. 5.36. The corners of the wings were rounded with $10 \%$ fillets shown in figure 21.

$$
A R=\frac{z}{c}
$$




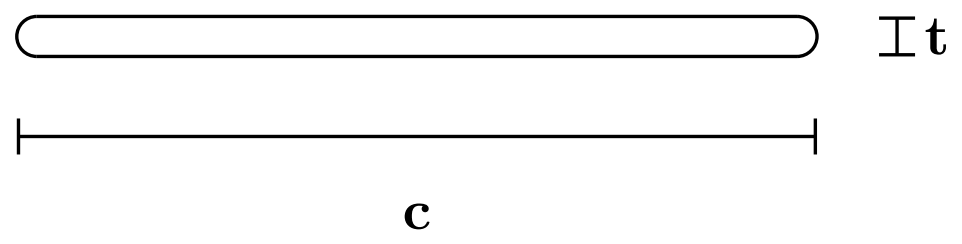

Figure 20: Flat plate profile with a chord $(c)$ of one, and $5 \%$ chord thickness $(t)$

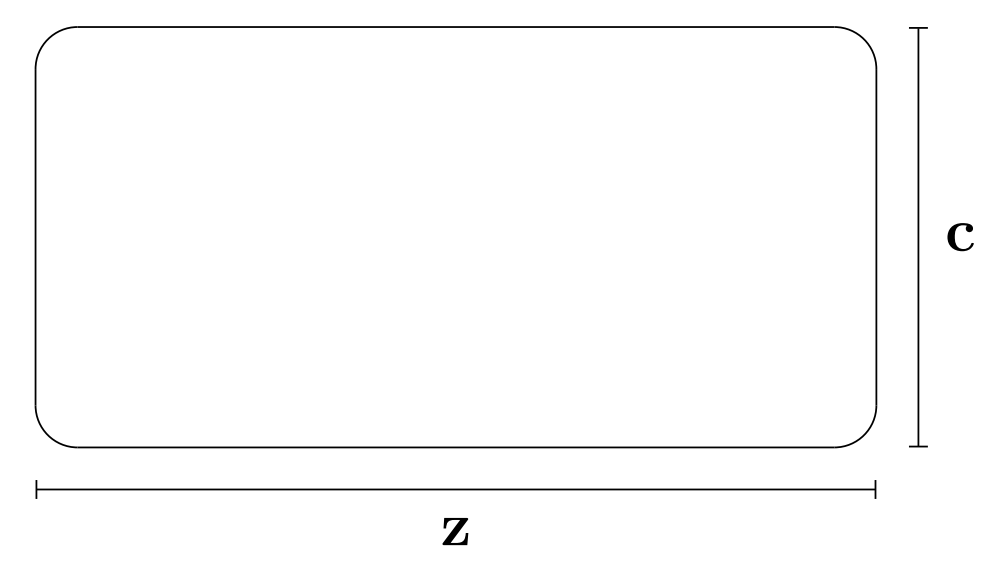

Figure 21: Planform of the test wing gemoetries where $z$ is the span length.

The flat plate wing was represented by a single body fitted grid in the 2D cases, and by three separated body fitted structured grids (wing body grid, and two wing tip grids) overlapped together for the 3D case. This can be seen in figure 22 .

This composite wing grid was then placed inside a uniform Cartesian background grid. Typically the background grid is usually much coarser than the wing grid. When the grid size ratio in the overlapping region is large, a large error due to interpolation occurs. To minimize the interpolation error, one or multiple intermediate grids can be added to link the background grid and the wing grid. In our study, two such grids are added and are marked with blue, green, and red in figure 23. 

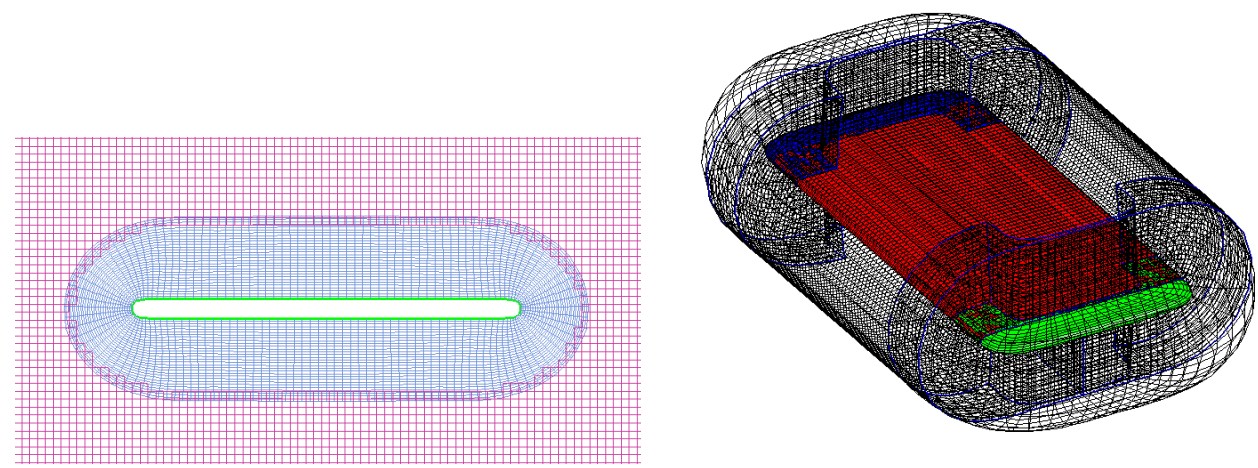

Figure 22: (Left) 2D body fitted grid. (Right) 3D composite grid.
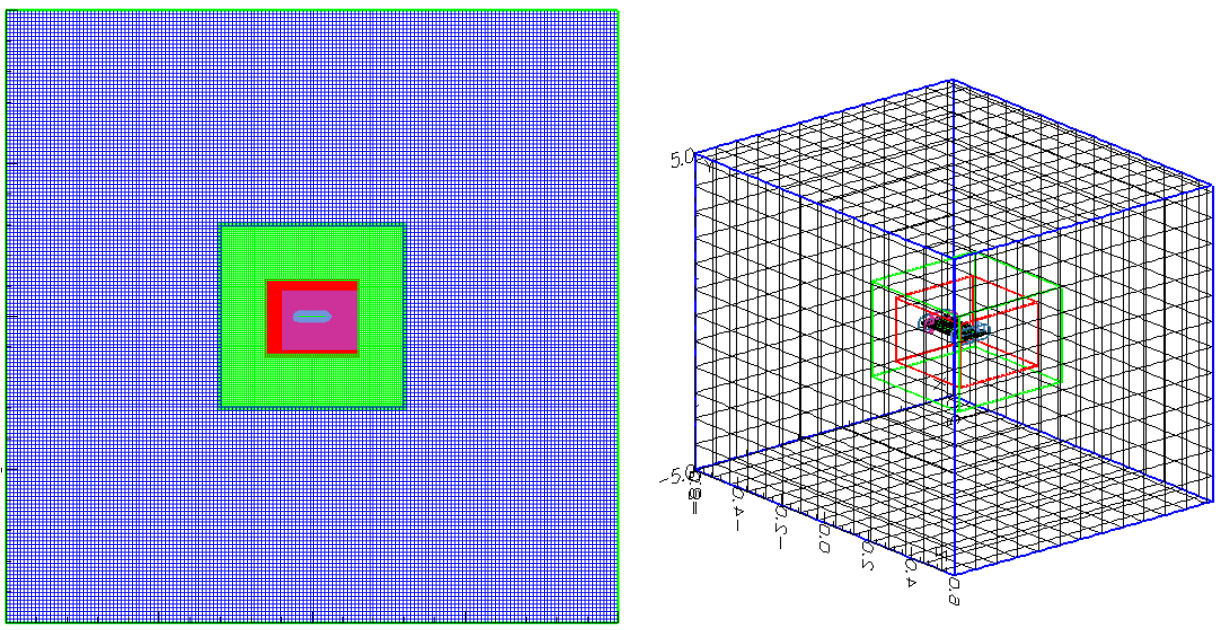

Figure 23: (Left) 2D and (right) 3D grid domains. 


\section{Domain Size}

Before testing each perching maneuver, the background grid must be properly sized first. In an experimental study by Granlund et al. [8], showed the effects of small domains sizes have on coefficient of lift in water channels. They found the coefficient of lift increased by a factor of two when the domain was halved in size by doubling the chord length of the airfoil. This effect however was not prominent until high angles of attack. The problem primarily stemmed from no-slip wall effects inside of water tunnel. This showed that if a domain was not of sufficient size, the velocity of the fluid at the center of the channel would be sufficiently higher than at the entrance thus increasing lift. Also at high angle of attack, the airfoil can create blockage effects which further exacerbate the problem. In numerical simulation it is possible to mitigate this effect by setting the top and bottom walls to inflow boundaries. In a previous study by Hord and Lian[68], the boundary size was also studied using the Overture framework looking at static wings, and the results are detailed here. They looked at a corrugated airfoil at $20^{\circ}$ and tested three domain sizes, 5c, 10c, and 20c.

From their study, it was found that if the domain walls were at least 10 chords away from the surface of the wing, and boundaries were set as outlet conditions, then there were no blockage effects.

\section{Grid Sensitivity}

In this section, only the the results of the flat plate wing with an aspect ratio $(A R)$ of 2 , and the reduced pitch rate $(K)$ of 0.2 are discussed here. The simulation was conducted at a Reynolds number of 500, and the flow was assumed to be laminar. Three grid densities, referred to Coarse, Medium, and Fine, were tested. The grid density increased by $20 \%$ in each direction from Coarse to Medium and then from Medium to Fine, resulting in a grid size of $922 \mathrm{k}, 1.7 \mathrm{M}$, and $2.8 \mathrm{M}$ for the Coarse, Medium and Fine grid system, respectively. Table 2 lists the three test cases with 
the number of points used to define the airfoil and span cross-sections, the initial cell height, the total grid points in the domain, and the total time to compute the simulation.

\section{TABLE 2}

Three grids used in the grid sensitivity analysis

\begin{tabular}{|c|c|c|c|c|c|}
\hline Grid Quality & Airfoil & Span & $\begin{array}{c}\text { Initial Cell } \\
\text { Height }\end{array}$ & $\begin{array}{c}\text { Total Grid } \\
\text { Points }\end{array}$ & $\begin{array}{c}\text { Time } \\
\text { (Hours) }\end{array}$ \\
\hline Coarse & 72 & 80 & $6.65 \times 10^{-3}$ & 922,535 & 42 \\
\hline Medium & 90 & 100 & $5.54 \times 10^{-3}$ & $1,705,272$ & 155 \\
\hline Fine & 108 & 120 & $4.46 \times 10^{-3}$ & $2,834,216$ & 460 \\
\hline
\end{tabular}

Figure 24 shows the computed lift and drag histories for each case. Lift and drag from the Medium grid shows little variance from the Fine grid, while the Coarse grid shows significant under prediction. The wake velocities were also examined to verify convergence. The sampling of the wake velocities were taken at two locations: (1) at the mid-span a quarter-chord behind the wing in the vertical direction and (2) along the span direction of the wing, a quarter-chord behind the wing. Both sets of wake velocities are reported in figure 25. Little difference was found in the velocity distributions, suggesting that the velocity field is adequately resolved by the Medium grid.

The computational time required to solve each case is important to take in consideration. Minor changes in grid density of $20 \%$ in each direction resulted in a near doubling of global node count. This increased computational time by a factor of four. Thus the Medium grid was selected as it provides a resolved velocity field and little degradation to the aerodynamic forces compared to the Fine case. Additionally, the Fine case requires three times the computation time compared to the Medium grid.

Reduction in computational cost could be achieved by simulating half the wing with a symmertric boundary condition at the half span. Based on the sensitivity 
results, this may reduce the time required by a factor of three to four times. However, issues were found with the overlapping of each refinement grid sharing the same face. Thus simulating only the half span was not pursued.
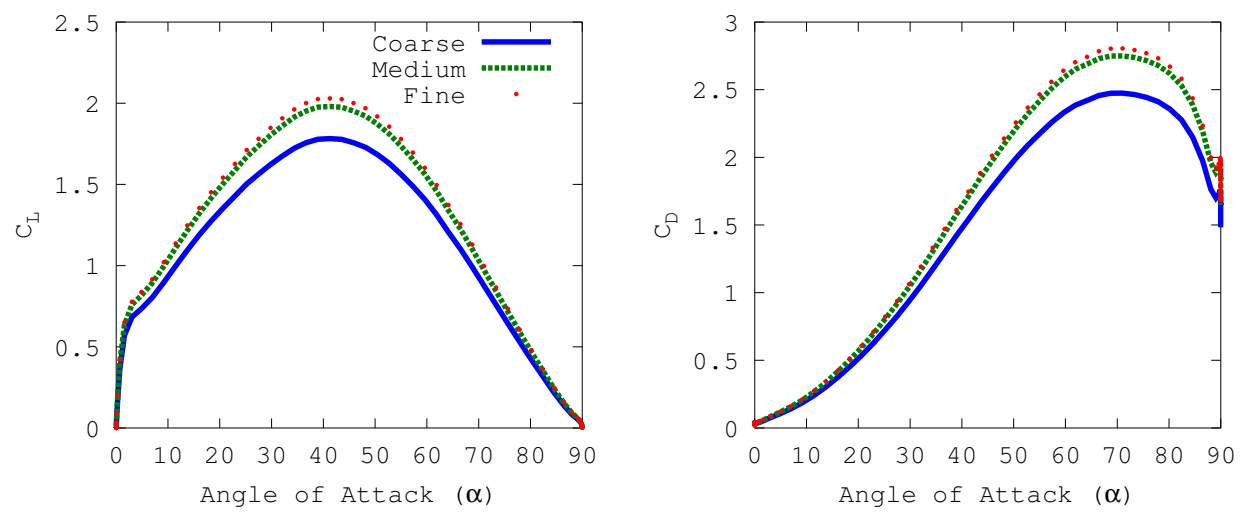

Figure 24: Aerodynamic forces for the grid sensitivity analysis of a pitching wing of $\mathrm{AR}=2, \mathrm{~K}=0.2$, and $\mathrm{Re}=500$. 

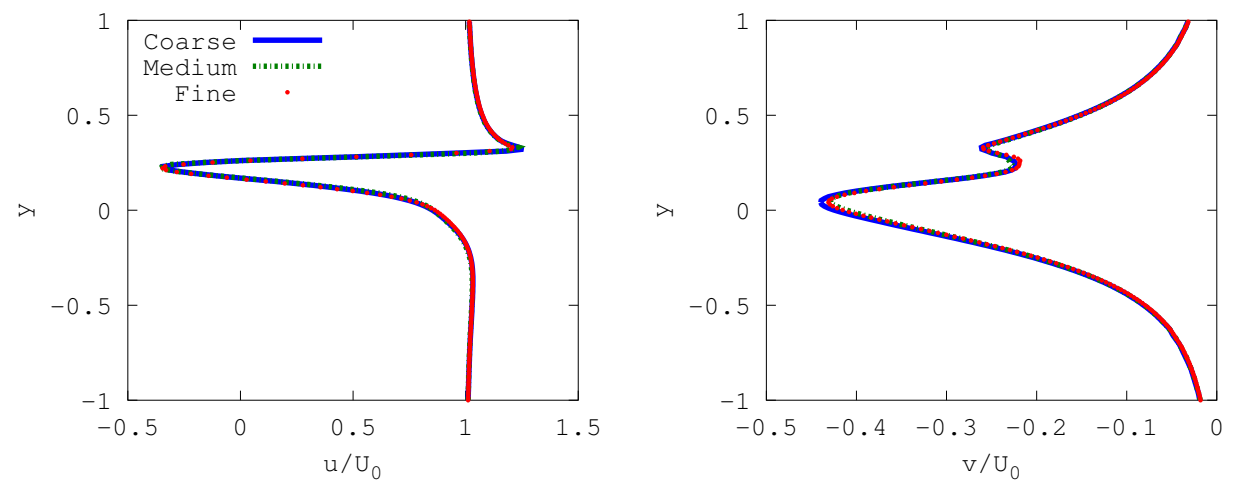

(a) Vertical velocity distribution in the y-direction
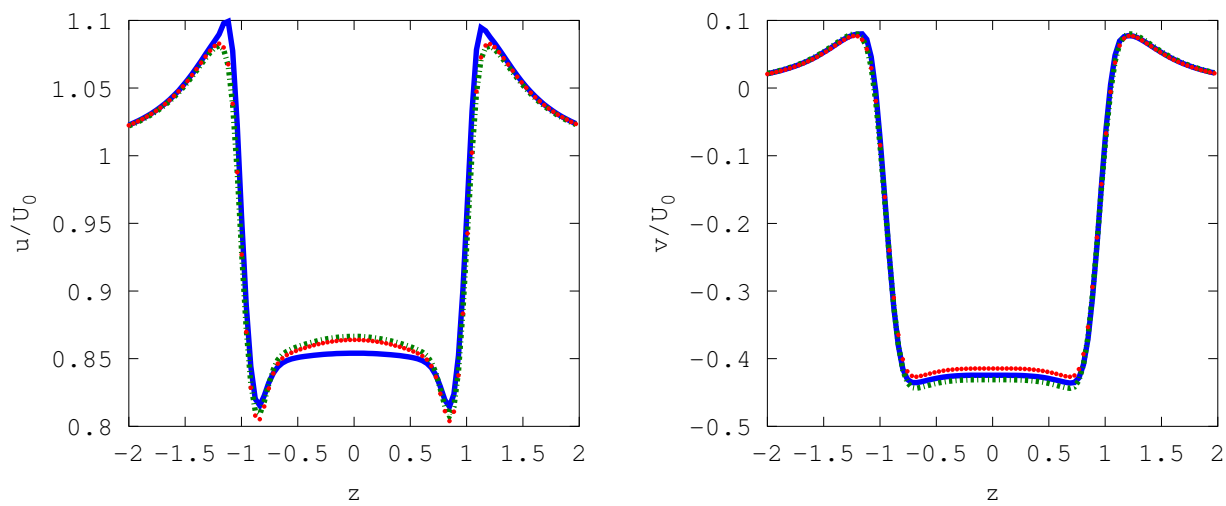

(b) Spanwise velocity distribution in the z-direction

Figure 25: $\mathrm{U}$ and $\mathrm{V}$ velocity distributions quarter chord behind the airfoil at $45^{\circ}$. 


\section{CHAPTER VI}

\section{AERODYNAMIC ANALYSIS}

\section{A. Introduction}

As stated previously, the purpose of this work is to investigate the effect of aspect ratio and reduced pitch rate on the development of the LEV during the pitchup and perching process. Here the calculated aerodynamic forces of both 2D and 3D wings with aspect ratios of 1,2 , and 4 are compared to illustrate their respective effects. Three reduced pitching rates $(0.05,0.1,0.2)$ were also tested for the $2 \mathrm{D}$ cases, and the latter two for the 3D. In the following sections, the Reynolds number sensitivity, kinematic and geometry effects on aerodynamic forces, and vortical flow structures about the wing will be examined. It is worth repeating that unless otherwise specified, the simulations conducted here are at a Reynolds number of 500, and always assumed to be laminar.

Depending on the discussion of either the pitch-up or perching motions, the aerodynamic coefficients of lift and drag are normallized by differently. For the pitchup cases, the lift and drag coefficients are calculated with the previous stated equations 4.34 and 4.35 . The perching data is normallized instead by instantaneous velocity of the motion:

$$
\begin{aligned}
C_{L} & =\frac{F_{L}}{2 \rho A V_{i n s t}^{2}} \\
C_{D} & =\frac{F_{D}}{2 \rho A V_{i n s t}^{2}}
\end{aligned}
$$




\section{B. 2D Analysis}

Where $V_{i} n s t$ is the velocity at that angle of attack (figure 5). Normallizing be the instantaneous velocity results in dividing by a near zero and zero quantity at the end of the motion. This creates artifacts in the coefficient data that appear as the trend expodentially growing near the end of the motion.

\section{Effect of Reynolds Number}

To fully investigate the Reynolds number effect on the pitch-up motion, several Reynolds numbers simulations were performed. In this analysis, four Reynolds numbers: 500, 5000, 10,000, and 20,000 were examined using a $2 \mathrm{D}$ flat plate at $K=0.2$ but pitching around the quarter cord location.

Figure 26 compares the simulated lift and drag coefficients at the four different Reynolds numbers. It is clear that the forces vary with the Reynolds number. All tested cases have similar force profiles but the magnitude of the force coefficients varies with the Reynolds number. From figure 26 it can be seen that the lift slope and peak lift and drag coefficient increase with the Reynolds number but the stall angle decreases. Granlund et al. [8] experimentally studied the same pitch-up kinematics based on a wall-to-wall mounted flat plate but at a higher Reynolds number of 20,000. Their lift coefficients agree well with our results at $\mathrm{Re}=20,000$ but their drag coefficients are higher. (This was also seen previously when the pivot point was at the leading edge in figure 19).

The initial spike at the beginning motion is the result of noncirculatory forces, predominately the rotational acceleration. Examining the lift spike at the beginning of the motion, the amplitude of the spike did not change with the Reynolds number. This suggests that at this stage, flow is dominated by noncirculatory forces due to the initial pitching acceleration at the start of the motion. The remaining discrepancy between the magnitudes of the force coefficients can be contributed to circulatory forces (aerodynamic forces) that depend on the Reynolds number. This conclusion is 
later verified in Chapter VII.
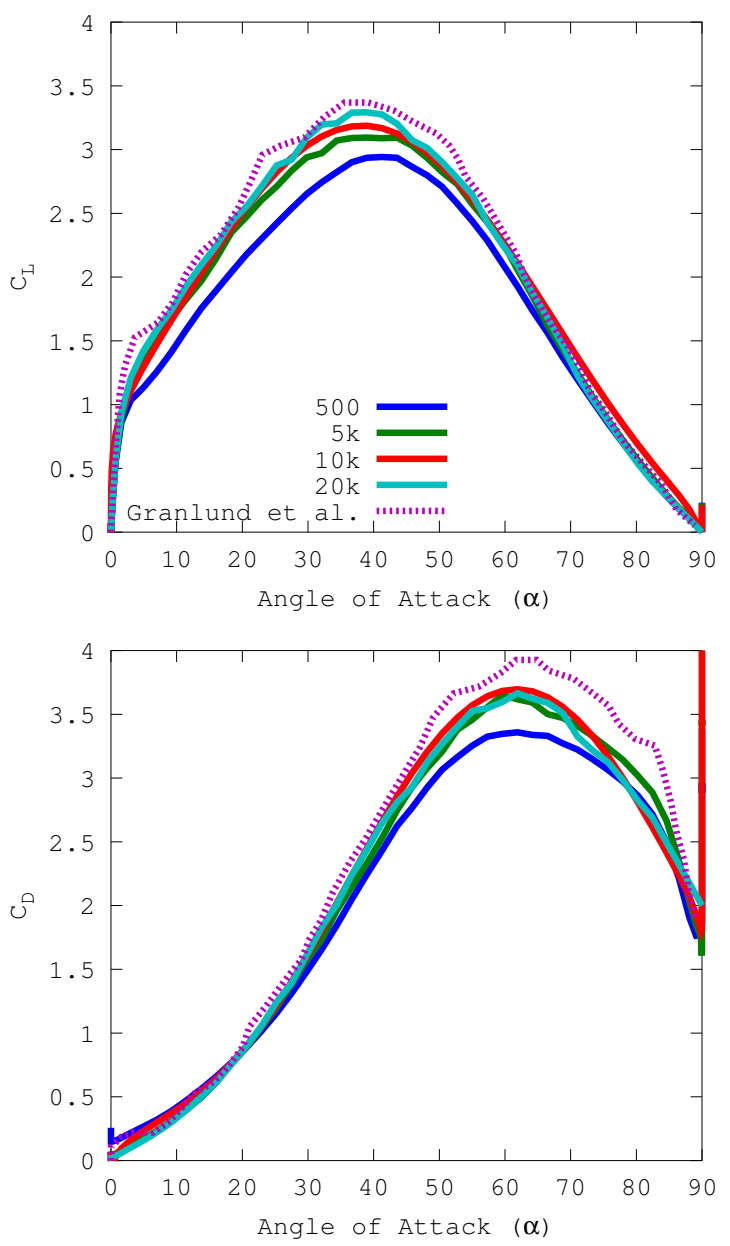

Figure 26: Reynolds number sensitivity of the $2 \mathrm{D} K=0.2$ pitch-up case tested at Reynolds numbers of 500, 5000, 10,000, and 20,000 compared to Granlund et al. $(\operatorname{Re}=20,000)[8]$

Figure 27 compares the vorticity contours at different Reynolds numbers at an angle of attack of $50^{\circ}$. This chosen angle is close to the stall angle for each case. At the lowest Reynolds number of 500, the formed LEV is dissipated compared to the higher Reynolds numbers. As the Reynolds number increases, the LEV becomes visually defined with stronger interior vorticity due to reduced dissipation effect of viscosity. Another feature shown in figure 27 is that as the Reynolds number increases 
small vortex structures appear and they are not damped out quickly due to viscous effects.

\begin{tabular}{cccc}
\multicolumn{4}{c}{ Reynolds Number } \\
500 & 5000 & 10,000 & 20,000 \\
\hline \hline
\end{tabular}
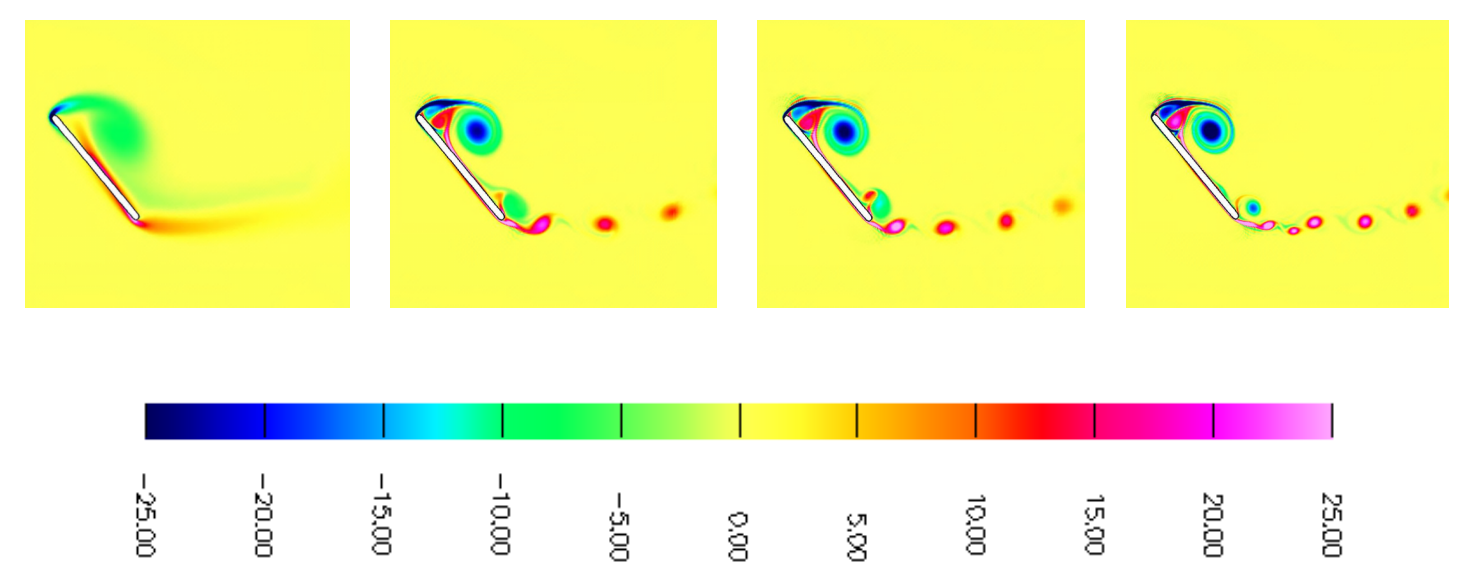

Figure 27: Change in vorticity due to Reynolds number sensitivity of the $2 \mathrm{D} K=0.2$ pitch-up case tested at Reynolds numbers of 500, 5000, 10,000, and 20,000.

\section{2D Pitch-up}

Continuing the pitch-up analysis, pitch rates of $K=0.05,0.1$, and 0.2 were tested at a Reynolds number of 500 with a pivot point at $\mathrm{x} / \mathrm{c}=0.25$. The instantaneous lift and drag coefficients are plotted in figure 28. It is clear that the coefficient of lift and drag, as with the stall angle all increased with pitch rate, which agrees with the experimental observations [8]. This increase in force coefficients can be attributed to the dynamic stall affect. Even though each motion is pitching through the saem angles of attack, the higher reduced pitch rates retain the LEV at higher angles of attach increasing lift duration. 

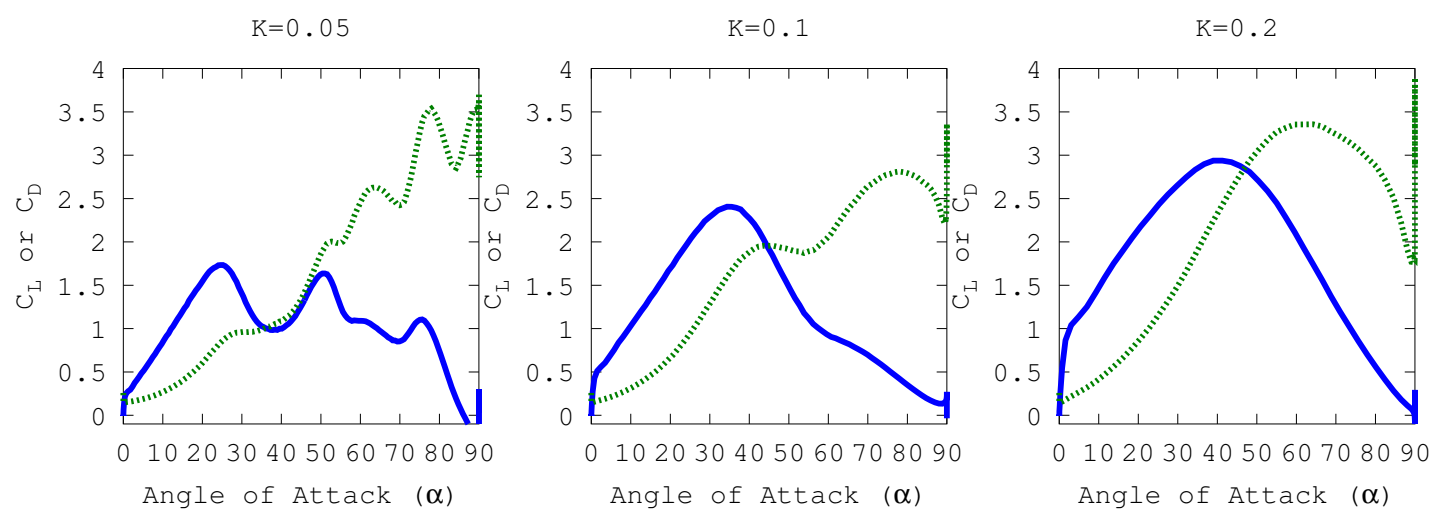

Figure 28: Coefficient of lift (solid) and drag (dotted) for the pitch-up cases at their respective instantaneous angles of attack.

Figure 29 illustrates this effect of pitch rate on the behavior of LEV. At the slowest pitch rate of 0.05 , the LEV forms fairly early on during the pitch-up motion and sheds quickly. As the pitch rate increases, the timing of the roll up of the LEV is delayed. This delayed development of LEVs extends the influence of LEVs over a wide range of angles. The LEV can remain tightly curled on the wings surface at higher angles with increasing pitch rate. Eventually the LEV does shed from the wing, resulting in stall and a decrease in lift. 


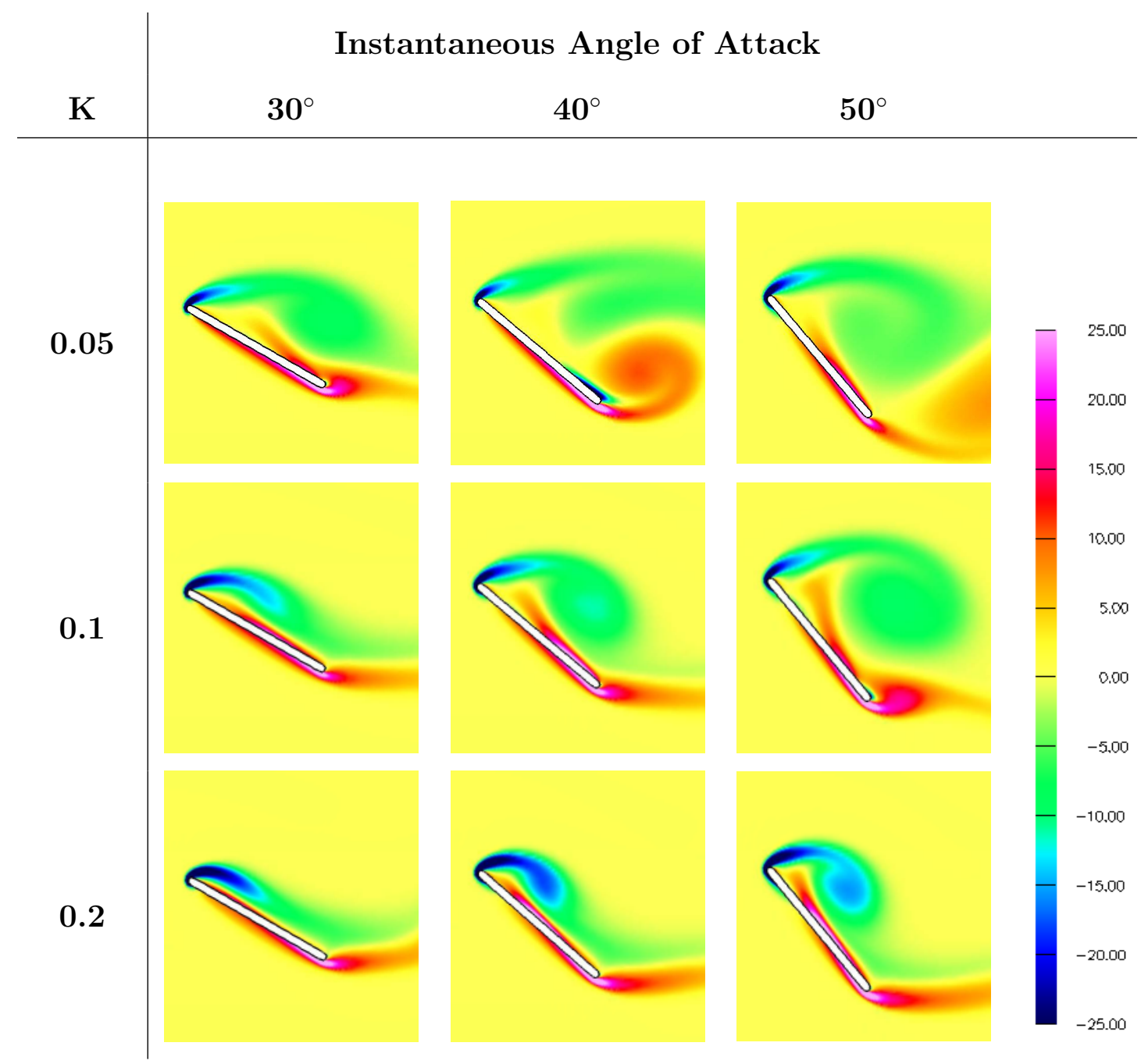

Figure 29: Vorticity contours at differing pitch rates and angles at pitch-up with a Reynolds number of 500 .

\section{2D Perching Wing}

To understand the impact of streamwise deceleration on the pitching wing (perching), the 2D aerodynamics are studied here. Similar cases were reported by Granlund et al. [8]. As a departure from their work, the coefficients are normalized by both the initial streamwise flow velocity and the instantaneous flow velocity as shown in figure 30. In the work of Granlund et al, the coefficients were only normal- 
ized by the instantaneous velocity. Both cases of normalization are shown to illustrate the differences between using instantaneous velocity and maximum velocity and different conclusions on perching can be drawn. When the coefficients were normalized by instantaneous velocity, the trends observed follow the experimental results [8]. Exceedingly large lift and drag values near the end of the perching motion are due to dividing by a near zero velocity value as shown previously in the kinematic plots (figure 5).
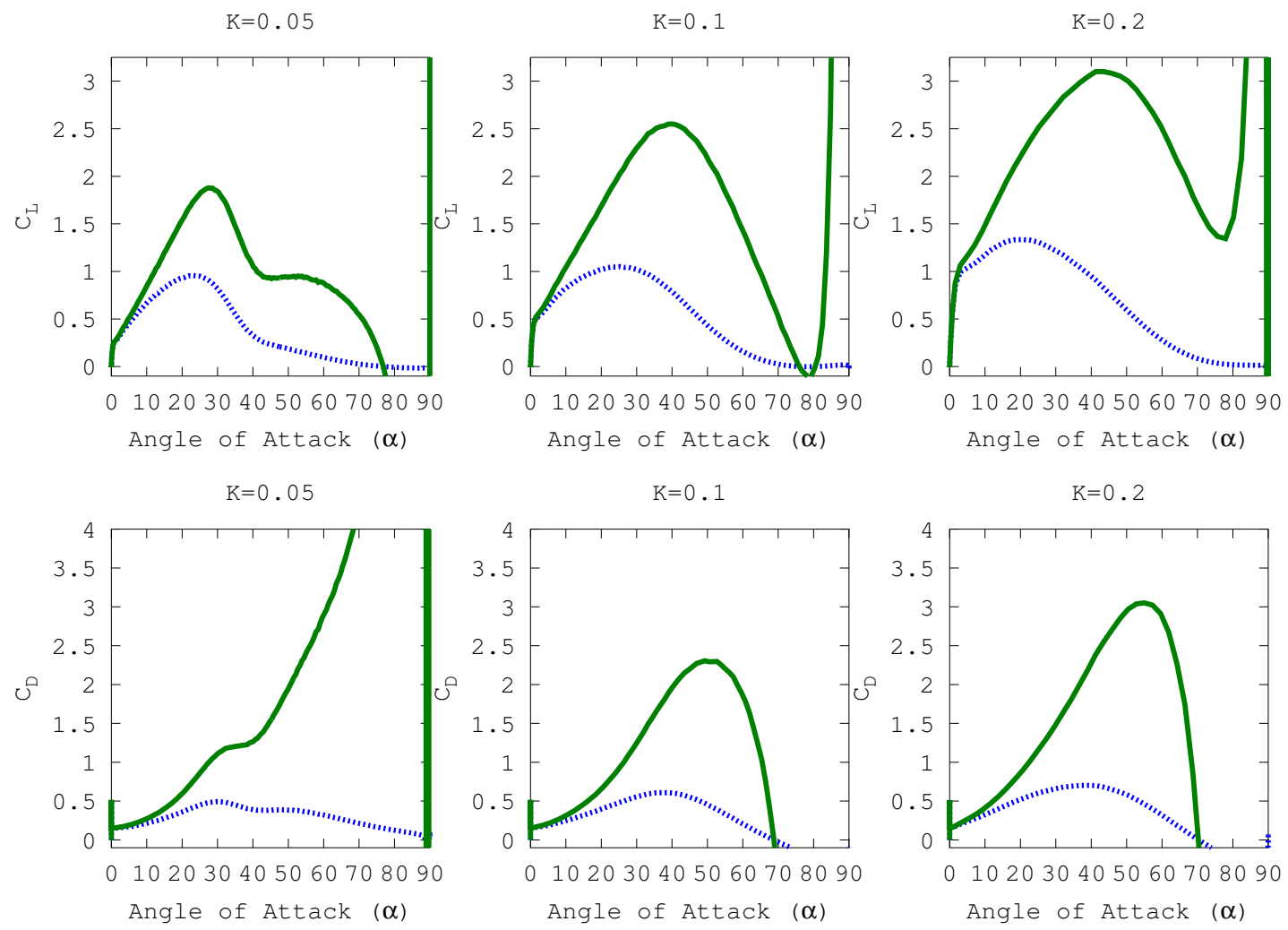

Figure 30: (Top) Coefficient of lift and (Bottom) coefficient of drag for perching cases normalized by initial instantaneous velocity (solid) and initial flow velocity (dotted) at their respective instantaneous angles of attack.

When normalized by instantaneous velocity Granlund et al.[8] concluded that comparing to the corresponding pitch-up case the streamwise deceleration (perching) 
increases stall angle and peak lift coefficient, which is consistent with the results normalized by the same instantaneous velocity. However, when normalized by the initial velocity the numerical results show that increasing pitch rate will still increases the lift coefficient, but will only decrease the stall angle when compared to its pitchup counterpart. Interestingly, the stall angle initially increases with pitch rate (from $K=0.05$ to 0.1 ), but then decreases with the pitch rate (from $K=0.1$ to 0.2 ).

These results can be summarized into two separate groups of thought:

- When normalizing by instantaneous velocity:

1. Pitch rate increases stall angle and maximum lift and drag.

2. Maximum lift coefficient exceeds the pitch-up case lift.

- When the results are normalized by maximum free stream velocity:

1. Pitch rate increases maximum lift and drag coefficients.

2. Stall angle initially increases then decreases with increasing pitch rate.

3. Overall magnitudes are lower compared to pitch-up cases.

4. Stall angles are lower compared to pitch-up cases.

The perching motion cannot be solely investigated through the examination of the force histories. As illustrated in the lift and drag plots for the pitch-up and perching motions, there exists a fundamental flow field difference betwen the two motions. Thus future investigations (Chapter VII) must examine the vortex structures around the perching wing to identify the changes in the flow when compared to a pitch-up case. 


\section{3D Analysis}

\section{Pitch-up and Perching Forces}

The aspect ratio effect was investigating by testing three flat plates with aspect ratio of 1,2 and 4 . Due to the high computational cost associated with the low frequency pitch rate case $(\mathrm{K}=0.05)$, only two pitch rates, $\mathrm{K}=0.1$ and 0.2 , are studied with a Reynolds number of 500. First the instantaneous lift coefficients are presented in figure 31 for the pitch-up cases. The 2D numerical results are also presented for comparison purpose. The following conclusions can be drawn from the graphs.

1. Before stall, a higher aspect ratio wing produces higher lift and drag coefficient than a lower aspect ratio wing.

2. The stall angle decreases with the increase of aspect ratio.

3. Similar to the $2 \mathrm{D}$ results, increasing pitch rate increases the stall angle.

4. As the aspect ratio increases, the lift curve approaches the $2 \mathrm{D}$ curves. 

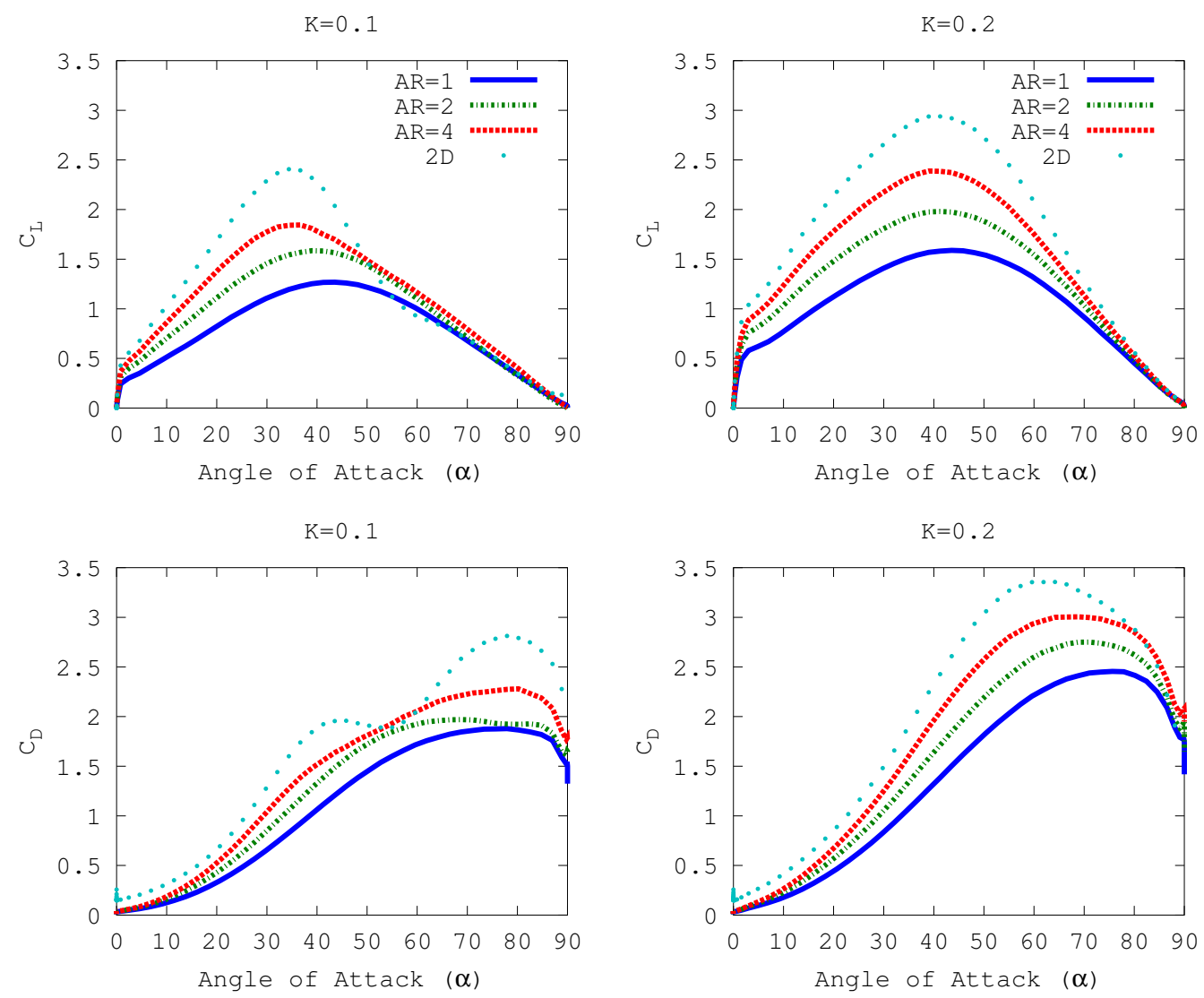

Figure 31: Comparison of lift and drag coefficients at different aspect ratios. (Pitchup, $\operatorname{Re}=500)$.

The results of perching cases are presented in figure 32. Here, the results were normalized my instantaneous free stream velocity. Many of the same trends are seen as before with the pitch-up results. The following conclusions are drawn:

1. The stall angle decreases with the aspect ratio.

2. Stall angle decreases with the pitch rate while perching.

3. The force history profiles approach the $2 \mathrm{D}$ ones as the aspect ratio increases.

If the results were normalized by instantaneous velocity, the trends would be very similar to that of the $2 \mathrm{D}$ perching cases with the augmentation of magnitudes of each aspect ratio. 

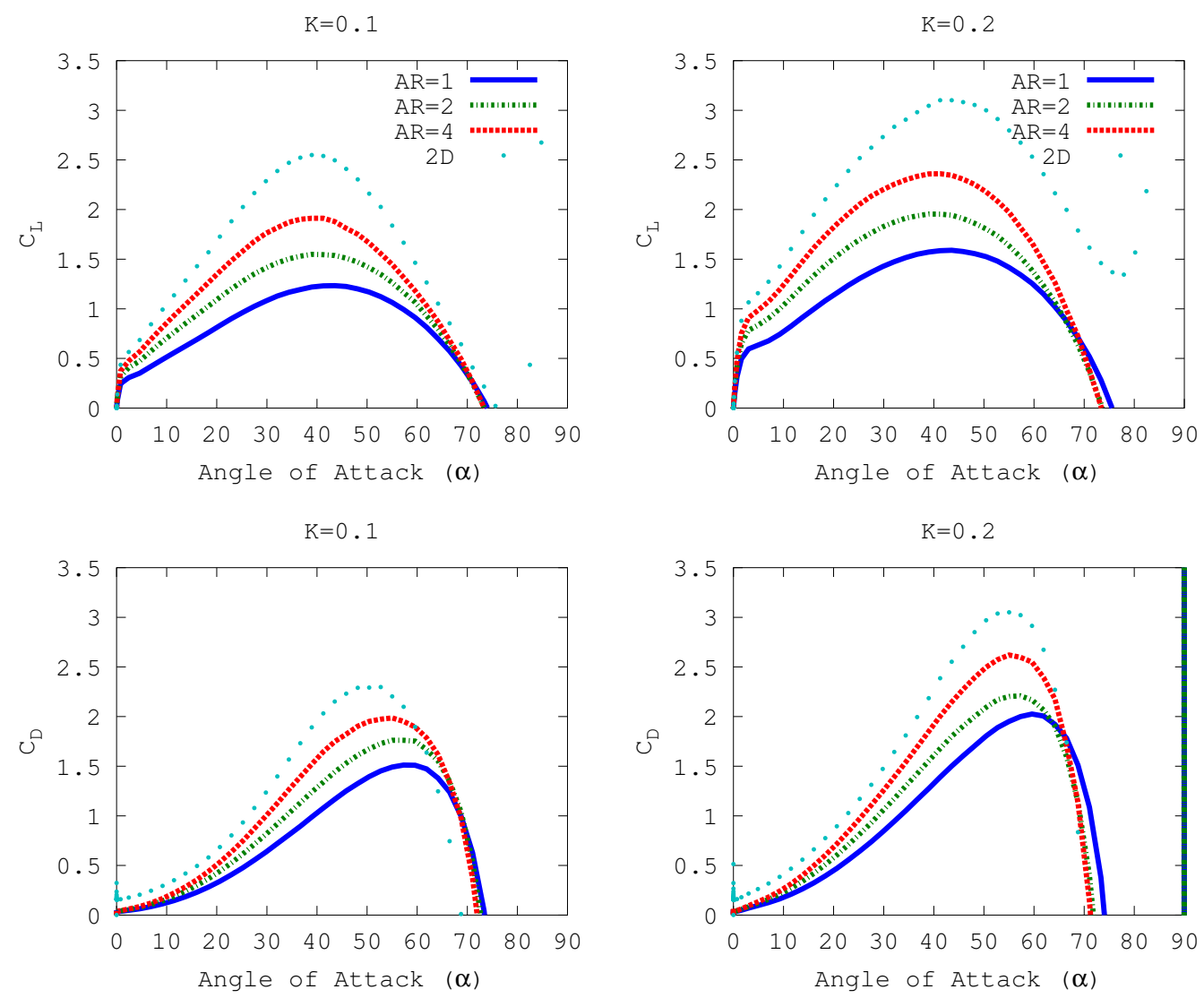

Figure 32: Comparison of lift and drag coefficients at different aspect ratios. (Perching , $\mathrm{Re}=500)$.

\section{Aspect Ratio}

As seen in figure 31 and figure 32, the lift slope was highly dependent on aspect ratio. As the aspect ratio increased, the lift slope followed. Figure 33 compares the LEV structure at the middle-span of different plates at an angle of attack of $45^{\circ}$ and $\mathrm{K}=0.2$. It is clear that as the aspect ratio increases, the LEV structure becomes more coherent, and gradually approaches the $2 \mathrm{D}$ case, which was true for all pitch rates tested. 


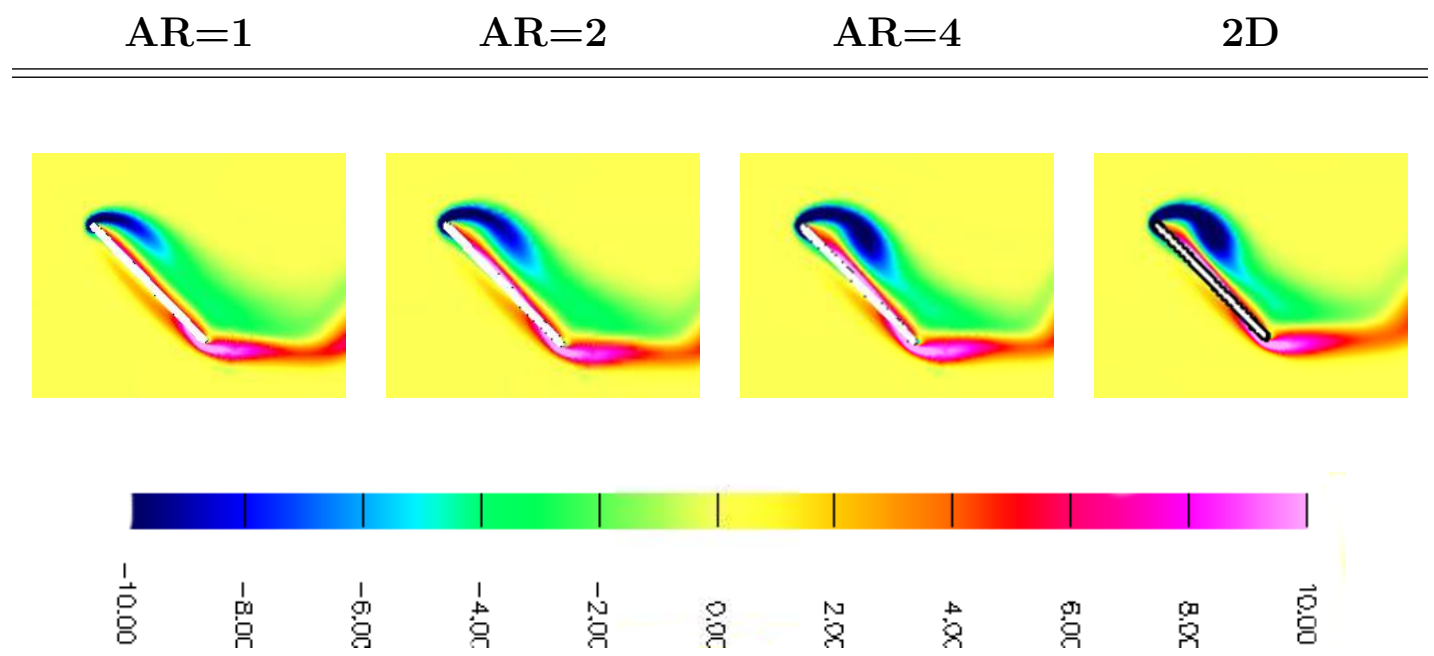

Figure 33: Vorticity contours at the mid-span of pitching plates with varying aspect ratios (pitching case, $\mathrm{K}=0.2, \mathrm{AoA}=45^{\circ}$ )

The effects of aspect ratio on the LEV has been observed before. Cosyn and Vierendeels [69] also saw weak LEVs in computational results of static flat plate wings at various aspect ratios of 0.5 to 2 . They found that the strength of the LEV on the wings with an aspect ratio of less than 1.5 was damped by tip vortices; however as aspect ratio increased the influence of tip vortices decreased and LEV became stronger. Torres and Mueller[42] studied static wings with varying aspect ratios and planforms. They found that as the aspect ratio increased, the lift slope approached the theoretical thin airfoil prediction. Torres [70] commented that tip vortices played an important role in force production for static low aspect ratio wings. Shyy et al. [71] numerically conducted an analysis on a hovering flat plate at a Reynolds number of 100, and saw that tip vortices also play an important role in lift production and could enhance lift produced depending on the kinematics of the flapping motion.

From figure 31 and figure 32, it can be seen that regardless of pitch rate or perching, the lift slope increases as the aspect ratio increases. As the aspect ratio changes, the lift slope approaches the $2 \mathrm{D}$ result. It is also possible to see that the lift slope is insensitive to the pitch rate which is consistent with the conclusion of 
Granlund et al. [24] that pitch rate effects are linearly additive to the circulatory forces. Thus it is plausible that the changing lift slope is due to LEV and tip vortex interactions. At low aspect ratios, tip vortices dampen the strength of the LEV more than at higher aspect ratios.

It is clear that increasing the aspect ratio has several impacts on the force histories. Encompassing all the changes observed, all peak force coefficients and associated slopes before stall were seen to increase with aspect ratio slowly approaching their 2D counterparts. Stall angle was the only quantity observed to be inversely proportional to the change in aspect ratio. Table 3 summarizes some of these changes for easier comparison.

Figure 34 shows the vorticity contours at the mid-span of the three tested wings at both $K$ values. Due to the diffusive nature of low Reynolds number flows, it is difficult to distinguish the boundaries of the vortices produced. The Q-criterion has been overlaid on the vorticity to define the edges of the vortices identified. At the same pitch rate, an increased vorticity magnitude extends further downstream above the surface of the wing as the aspect ratio increases. With the assistance of the Q-criterion, it can be inferred that the LEV is growing in both size and strength with aspect ratio. This however raises the question of the mechanism inducing the differences in vorticity structure observed in the plots. Discussed in a later section, it was found that tip vortices interact with the spanwise vorticity impeding LEV growth on the lower aspect ratio wings. 


\begin{tabular}{c|ccc}
\multicolumn{4}{|c}{$\mathbf{K = 0 . 1}$} \\
Aspect Ratio & Stall Angle $\downarrow$ & Peak $C_{L} \uparrow$ & Lift Slope $\uparrow$ \\
\hline \hline $\mathbf{1}$ & 42.40 & 1.27 & 0.029 \\
$\mathbf{2}$ & 38.96 & 1.58 & 0.038 \\
$\mathbf{4}$ & 38.97 & 1.82 & 0.048 \\
$\mathbf{2 D}$ & 34.37 & 2.40 & 0.064
\end{tabular}

\begin{tabular}{c|ccc}
\multicolumn{4}{|c}{$\mathbf{K = 0 . 2}$} \\
Aspect Ratio & Stall Angle $\downarrow$ & Peak $C_{L} \uparrow$ & Lift Slope $\uparrow$ \\
\hline \hline $\mathbf{1}$ & 43.55 & 1.58 & 0.031 \\
$\mathbf{2}$ & 41.26 & 1.98 & 0.039 \\
$\mathbf{4}$ & 38.97 & 2.38 & 0.046 \\
$\mathbf{2 D}$ & 41.26 & 2.93 & 0.062
\end{tabular}

TABLE 3

Effect of aspect ratio and $K$ on the pitch-up motion.

\section{Pitch Rate}

Increasing the reduced pitch rate $K$ has the following effects: (1) it increases the stall angle, (2) it reduces the peak drag angle, and (3) it increases the force and moment coefficient magnitudes. Figure 35 compares the vorticity contours at different angles between the two reduced frequencies for the $A R=4$ wing. At the same angles of attack, the LEV is smaller at $K=0.2$ than at $K=0.1$. The LEV stays closer to the wing attenuating both lift and drag at higher angles of attack. This does well to illustrate the influence of $K$ on peak lift and drag; however this does not provide an explanation into the developmental changes of the LEV. The vorticity strength of the LEV is seen to increase with the pitch rate, but that does not mean the LEV generates more lift producing circulation. To properly judge the influence of $K$ on 


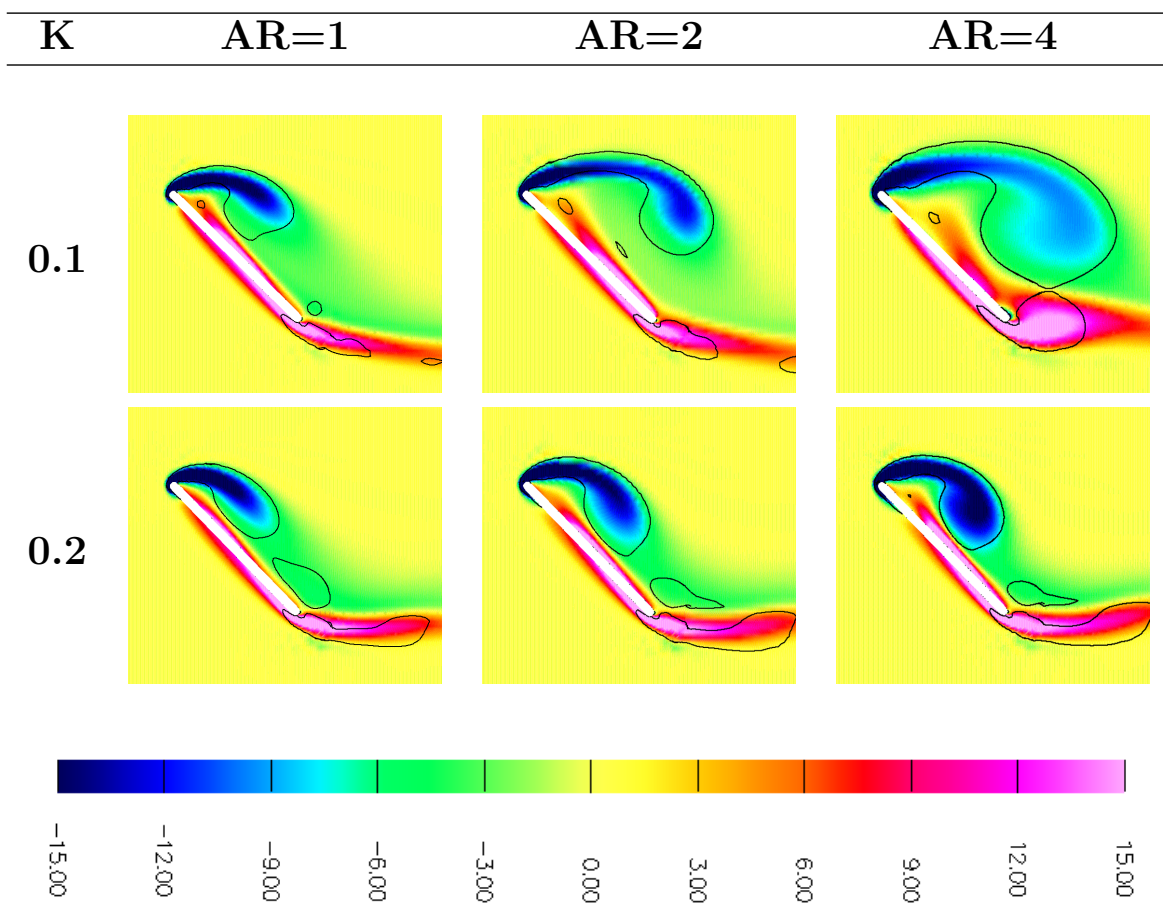

Figure 34: Comparison of the LEV vorticity contours at mid-span with the Q-criterion defining the boundaries of identified vortices. Snapshots were taken at $45^{\circ}$.

the LEV, the circulation is calculate and compared in Chapter VII.

\section{Perching}

Not explicitly addressed in the previous sections, has been the influence of the perching motion on the flow field and aerodynamic forces. All the previous conclusions of effects of aspect ratio and pitch rate can be applied to the perching motion. A key difference in the flow field is the resulting LEV formed and its propagation. Figure 36 plots the mid-span vorticity of the $A R=4$ wing for both values of $K$. Again the Q-Criterion is used to outline the areas of identified vortices.

The plotted vorticity remains on the same scale as figure 35, however the vorticity magnitudes are two to three times lower. The resultant identified vortices are also smaller in comparison. Both these effects are the result of the flow deceleration 


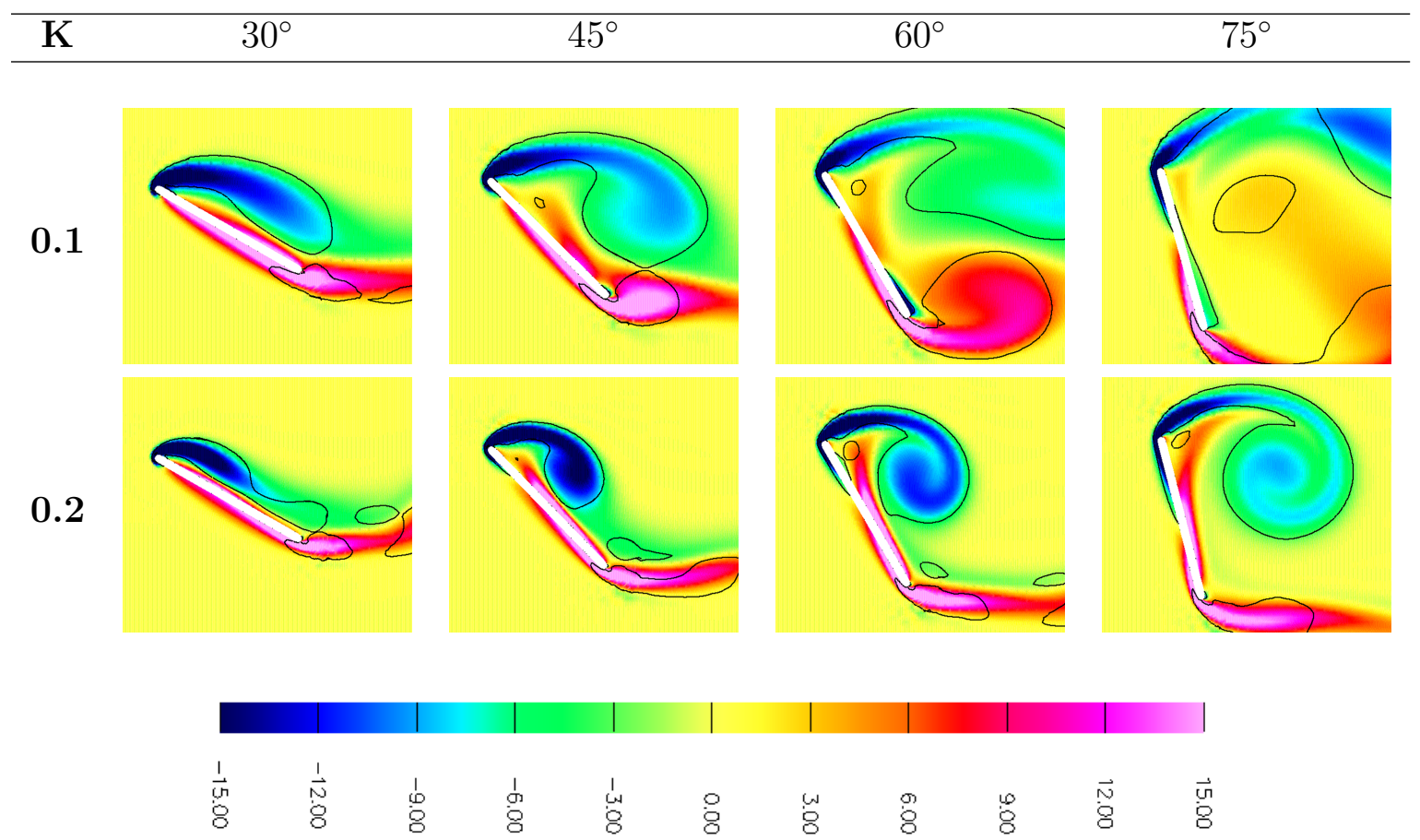

Figure 35: Comparison of LEV vorticity during the pitch-up motion of the $A R=4$ wing for $K=0.1$ and 0.2 with the Q-criterion defining the boundaries of identified vortices.

of the perching maneuver. With reduced velocity, less flow passes across the wing, resulting in the reduction of vortex development seen. Yet, due to the reduced flow velocity, the vortex remains closer to the wing even at higher angles of attack.

Previously in the pitching case (figure 35), the vortex had completely shed from the plate at $\alpha=75^{\circ}$ for the $K=0.1$ case, and was approximately three-quarters chord away from the wing with $K=0.2$. In the perching case, the vortex has moved little for $K=0.2$, and has began to move away from the plate for $K=0.1$. Perching is able to retain the proximity of the vortex leading to the possibility of continued lift and drag at higher angles of attack. 


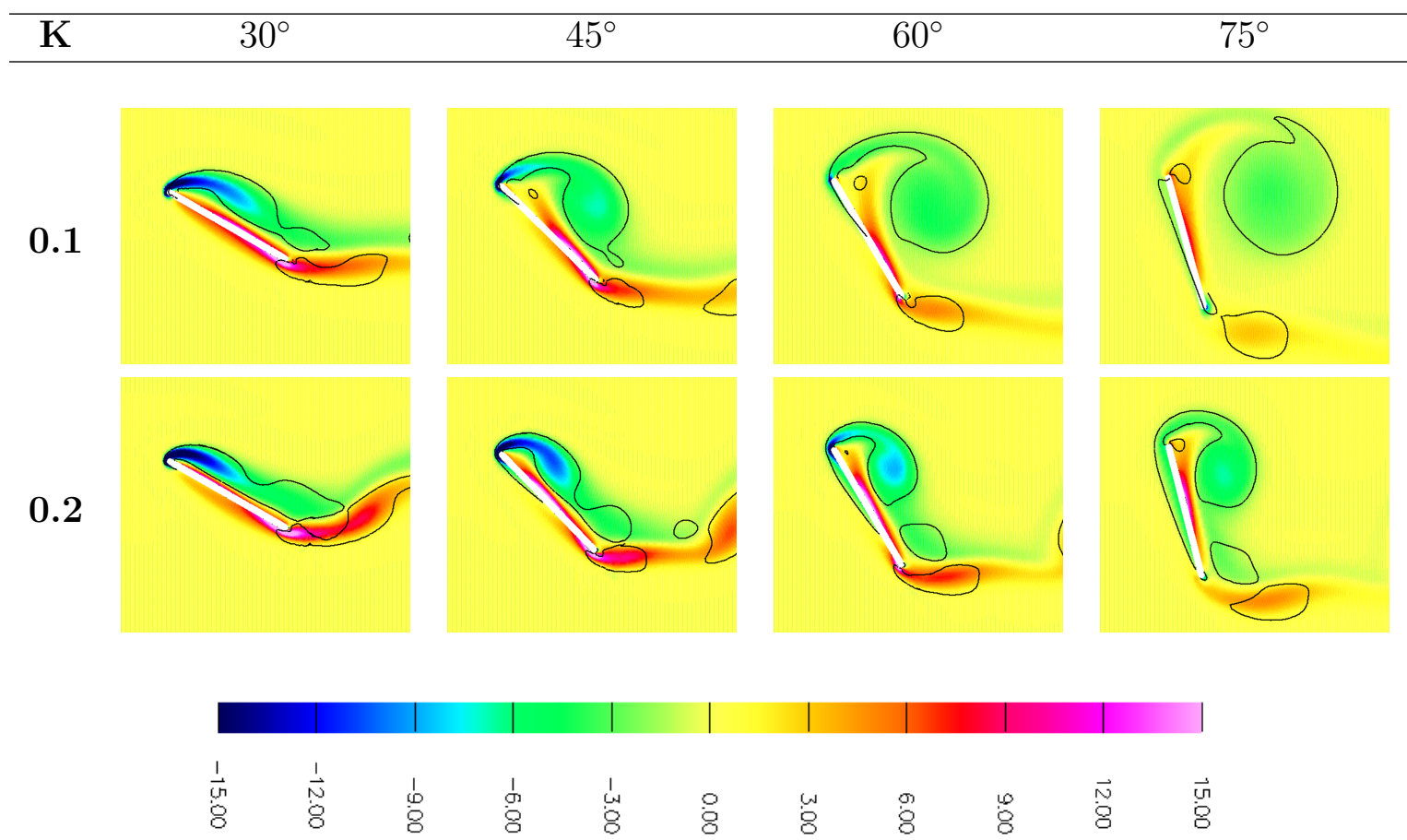

Figure 36: Comparison of LEV vorticity during the perching motion of the $A R=4$ wing for $K=0.1$ and 0.2 with the Q-criterion defining the boundaries of identified vortices.

\section{Spanwise Force Distribution}

The aspect ratio plays an important role in determining the resulting force distribution across the span of the plate. Figure 37 through 44 show various spanwise distributions of the normal force per unit span and vorticity iso-surfaces on wings of different aspect ratios. The normal force was chosen, as opposed to a force coefficient to avoid the bias influence of velocity normalization between the pitch-up and perching cases. It is important to note: the normal force is the resultant force of both lift and drag. Thus a higher normal force does not necessarily mean higher lift. The normal force along the span was calculated by integrating the pressure contour only around the chord at discrete span locations. 


\section{$\mathrm{K}=0.2$ Pitch-up}

In figure 37 it is clear that the distribution of the normal force is dependent on the aspect ratio of the wing. In fact, the force distribution across the span is not uniform, indicating flow is no longer $2 \mathrm{D}$ even on the higher aspect ratio wings. The non-uniformity across the span caused in the force distributions is mainly due to the presence of tip vortices.

At the angle of attack of $15^{\circ}$, the distribution of the force is fairly similar to one another among the tested three aspect ratios. A smooth arch along the span of all aspect ratios can be seen. At this angle, the force generated is primarily due to the LEV which is evident by the bulbous increase in lift at the center span and can be seen in the iso-surface contours in figure 38. The force drops to near zero near the tips due to the present of tip vortices. At this low angle, the strength and size of the tip vortices is small, but they still influence the force distribution.

As the angle increases to $30^{\circ}$, the force distribution changes, the LEV and the tip vortices become stronger. Corners have now appeared on the left and right sides of the span, showing the presents of the tip vortex. The amount the tip vortices affects the force over the span depends on the aspect ratio of the plate. The vortices across the aspect ratio 1 plate cover much of the surface on the suction side of the plate shown in figure 38. In comparison, tip vortices influence relatively smaller region on the $\mathrm{AR}=2$, and 4 plates. It can also be seen in figure 38 that the LEV has grown and changed shape significantly from the angle of attack of $15^{\circ}$. The tip vortices push parts of the LEV inward.

Increasing the angle further to $40^{\circ}$, the plate reaches the point where it begins to stall with the 0.2 pitch rate. Here the LEV has continued to grow in size and strength as with the tip vortices. The tip vortices have become more pronounced as horns on the distribution profile and now contribute greatly to the force generation, which is reflected by the spikes in the force near the tip regions shown in 37. From figure 38 the tip vortices cover more of the wing surface on the lower aspect ratio wing 
than on the higher aspect ratio wings. On the lower aspect ratio plate, tip vortices contribute largely to the force due to the suction side of the plate is being mainly dominated by the tip vortices. This is less true for the higher aspect ratio plates, where the sizes of the tip vortices are far smaller than the LEV.

Finally at $50^{\circ}$, the plate has passed its stall angle. The LEVs of on all the plates start to separate from the plates. For the aspect ratio 4 plate, the LEV sheds slower than the lower aspect ratio plates. This is indicated by the mound at the center span in 37. The aspect ratio 2 plate lacks this distinct feature, and aspect ratio of 1 plates LEV has been mainly pushed off by the tip vortices.
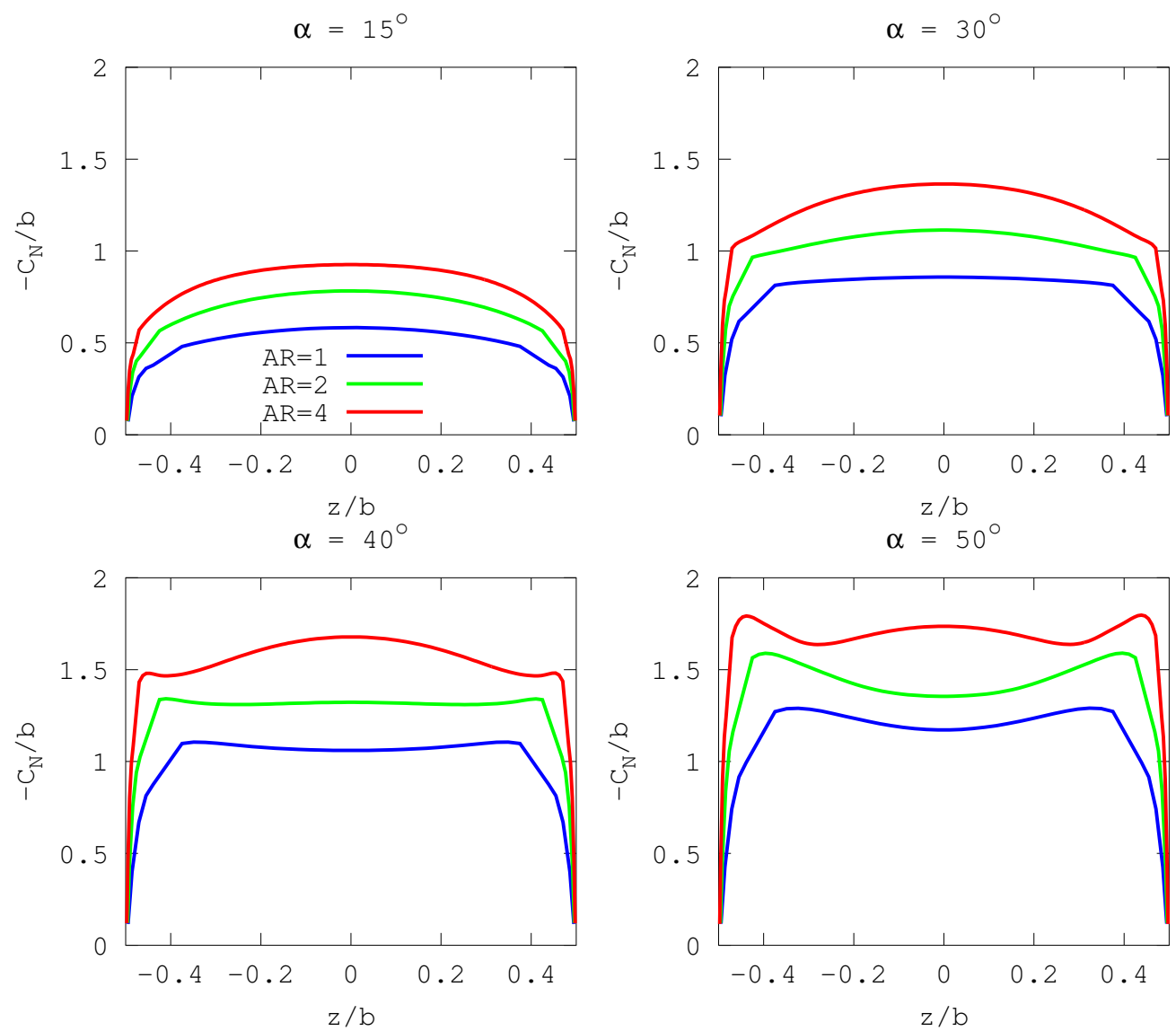

Figure 37: The normal force coefficient distribution plotted across the span of varying aspect ratios and angles of attack for the $\mathrm{K}=0.2$ pitch-up. 


\section{Pitch-up $\mathrm{K}=0.2$}

AR=1

Figure 38: Iso-surfaces of the Q-criterion $(Q=5)$ at discrete angles of attack of each aspect ratio at $K=0.2$. Views are in the upstream direction looking at the trailing edge of the wing. 


\section{$\mathrm{K}=0.1$ Pitch-up}

The spanwise distribution is further studied at the second pitch rate of $\mathrm{K}=0.1$, plotted in figure 39. The key differences between the $K=0.2$ case and the $K=0.1$ case are (1) the tip vortices are more pronounced and affect a larger portion of the span earlier on in the pitching process, which is because at lower pitch rate the tip vortices have more time to evolve into the inner span (2) stall occurs at a lower angle of attack, and (3) the LEV is weaker and sheds earlier.

Breaking down each individual angle, there is surprising similarity between the force distributions across the span at the angles of $15^{\circ}$ and $30^{\circ}$ when compared to the $\mathrm{K}=0.2$ pitch-up case. The shapes of the distributions are the same, but one has to remember actual forces are lower due to the reduced pitch rate.

At the higher angles, the horns created by the tip vortices are much broader and smoother. This is indicative to much larger and mature tip vortices as it can be seen in the iso-surfaces (figure 40). Finally, due to the earlier shedding of the LEV,

there force distribution is much lower at the center span when compared to $K=0.2$ pitch-up case. 

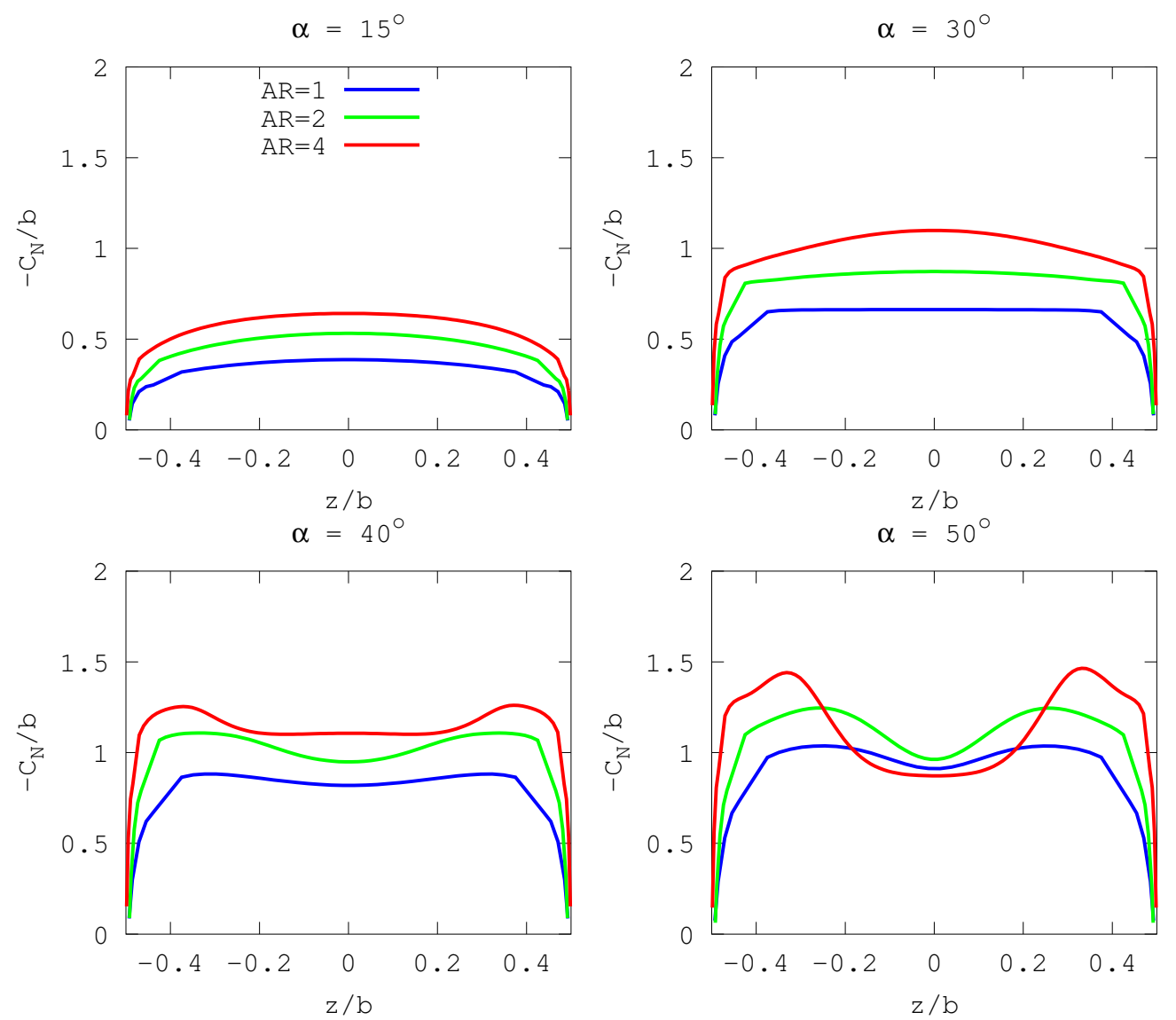

Figure 39: The normal force coefficient distribution plotted across the span of varying aspect ratios and angles of attack for the $\mathrm{K}=0.1$ pitch-up.

\section{$\mathrm{K}=0.1$ and $\mathrm{K}=0.2$ Perching}

Much like the force distribution plots for the pitch-up cases, the same plots have been produced for the perching cases in figure 43 and figure 44. Images of the 3D Q-Criterion are also shown in figure 46 and 46. The Q-Criterion is set to a lower value of 1 , due to the lower vorticity magnitudes seen in figure 36 .

It can be an ambitious task to compare all plots and visualizations together at once detailing the differences in force distributions and flow field. Thus a second set of plots were devised. The 3D pitch-up and perching results were plotted together 


\section{Pitch-up $\mathrm{K}=0.1$}

(15

Figure 40: Iso-surfaces of the Q-criterion $(Q=5)$ at discrete angles of attack of each aspect ratio at $K=0.1$. Views are in the upstream direction looking at the trailing edge of the wing. 
at their respective angles and pitch rates. The normal force values were normalized by their maximum value that occurs along the span for each respective case. This effectively scales each plot to fit between an arbitrary scale of 0 and 1 , yet allows for easier illustration of the difference in force distribution shapes between each of the individual cases. These new plots are reported in figure $41(K=0.2)$ and figure 42 $(K=0.1)$. (The true $C_{N}$ values are reported in figures 43 and 44$)$ The pitch-up values are represented by solid lines and the perching by dashed. One must remember these are normalized values, and are not representative of the true magnitudes of the actual measured force. These plots best represent the distribution of forces contributed by the LEV and/or tip vortices. 

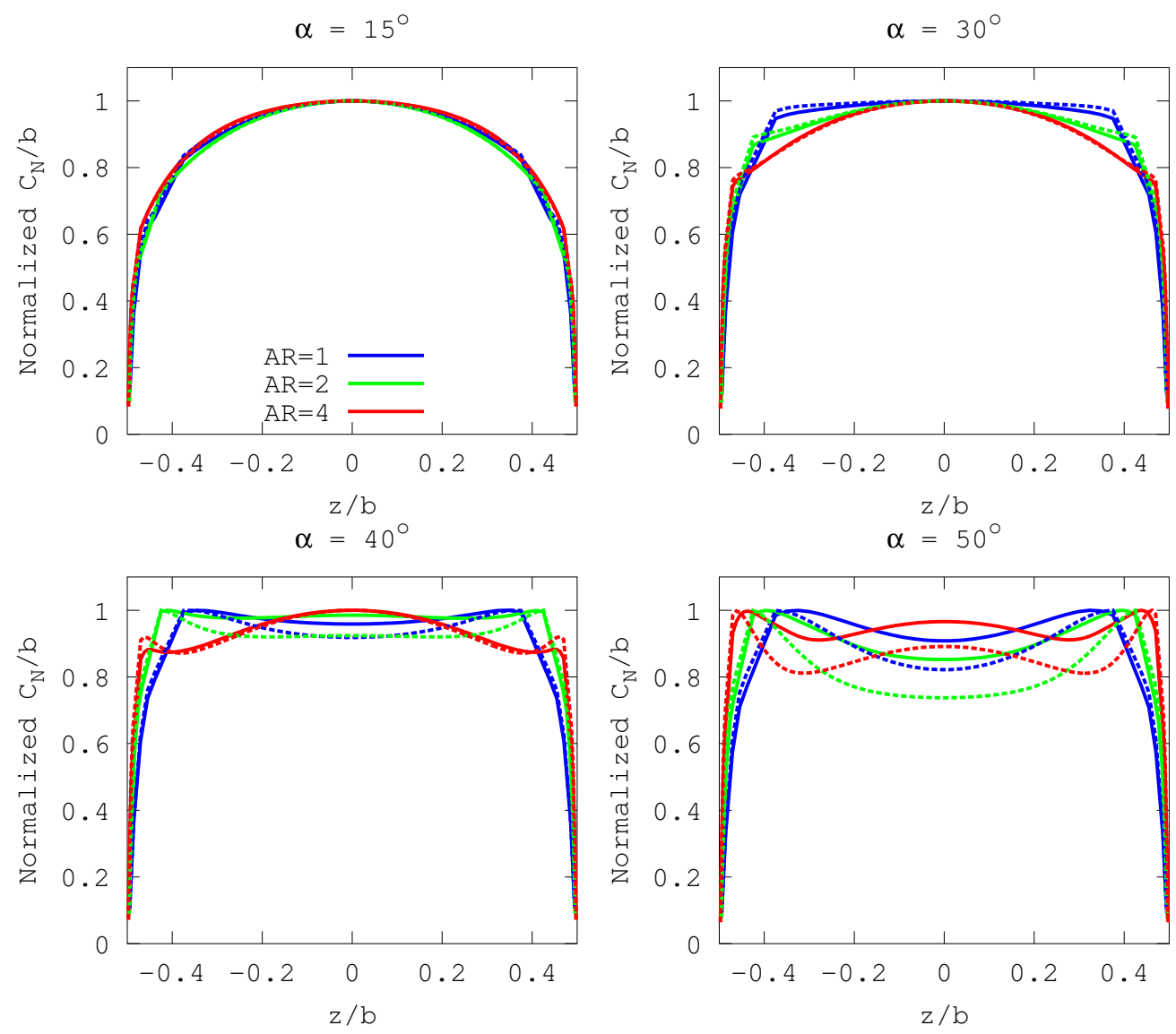

Figure 41: Normalized normal force distributions along the span of various aspect ratios for the pitch-up (solid) and perching (dashed) cases at $\mathrm{K}=0.2$. 

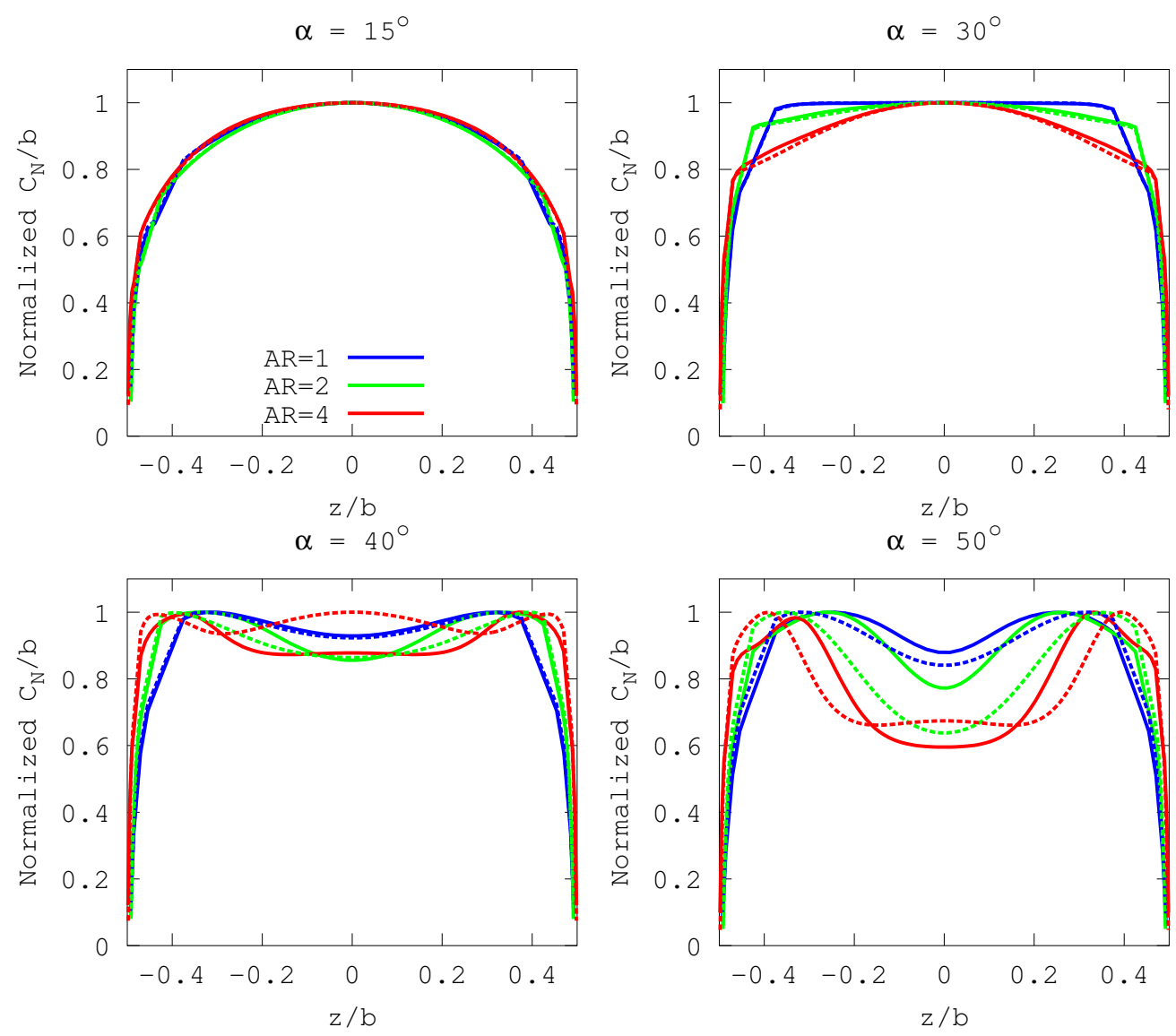

Figure 42: Normalized normal force distributions along the span of various aspect ratios for the pitch-up (solid) and perching (dashed) cases at $\mathrm{K}=0.1$.

For both tested $K$ values, little difference was seen in the shape of the force distribution when compared to their respective pitch-up case at angles $15^{\circ}$ and $30^{\circ}$. The primary difference was the reduction in magnitude of the forces seen in figure 43 and figure 44. At these angles, the free stream velocity has reduced approximately $16 \%$ and $33 \%$ respectively. Resulting in the similar flow distribution at the beginning of the motion.

Differences in the span appear at $40^{\circ}$. For the perching case of $K=0.2$, the LEV force contribution is lower, suggesting either a weaker vortex or earlier shedding for the lower aspect ratios, but is unaffected at the higher aspect ratio. The lower 
pitch rate of $K=0.1$, shows a similar trend; however tip vortices on the higher aspect ratio have moved inward as much compared to the pitch-up case.

At $50^{\circ}$, well passed stall, there is a lower center span force contribution. The weakened LEV, due to decreased velocity, has shed on the lower aspect ratio cases, while the higher aspect ratio remains close to the surface of the plate. For $K=0.2$, tip vortices again have not moved as far inward as before, but are evident on the aspect ratios of 2 and 4 plates. This is also true for the $K=0.1$ cases.
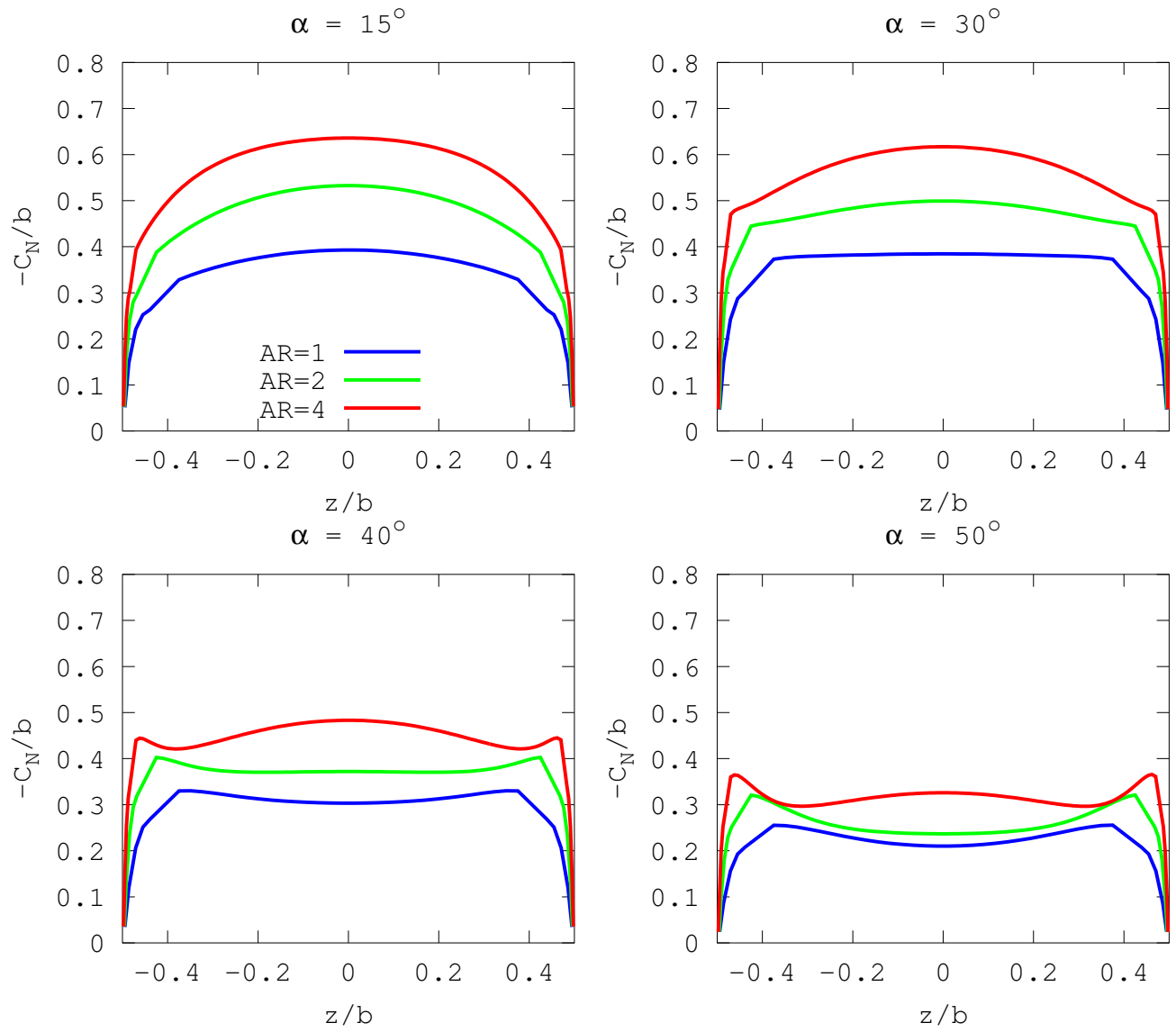

Figure 43: The normal force coefficient distribution plotted across the span of varying aspect ratios and angles of attack for the $\mathrm{K}=0.2$ perching. 

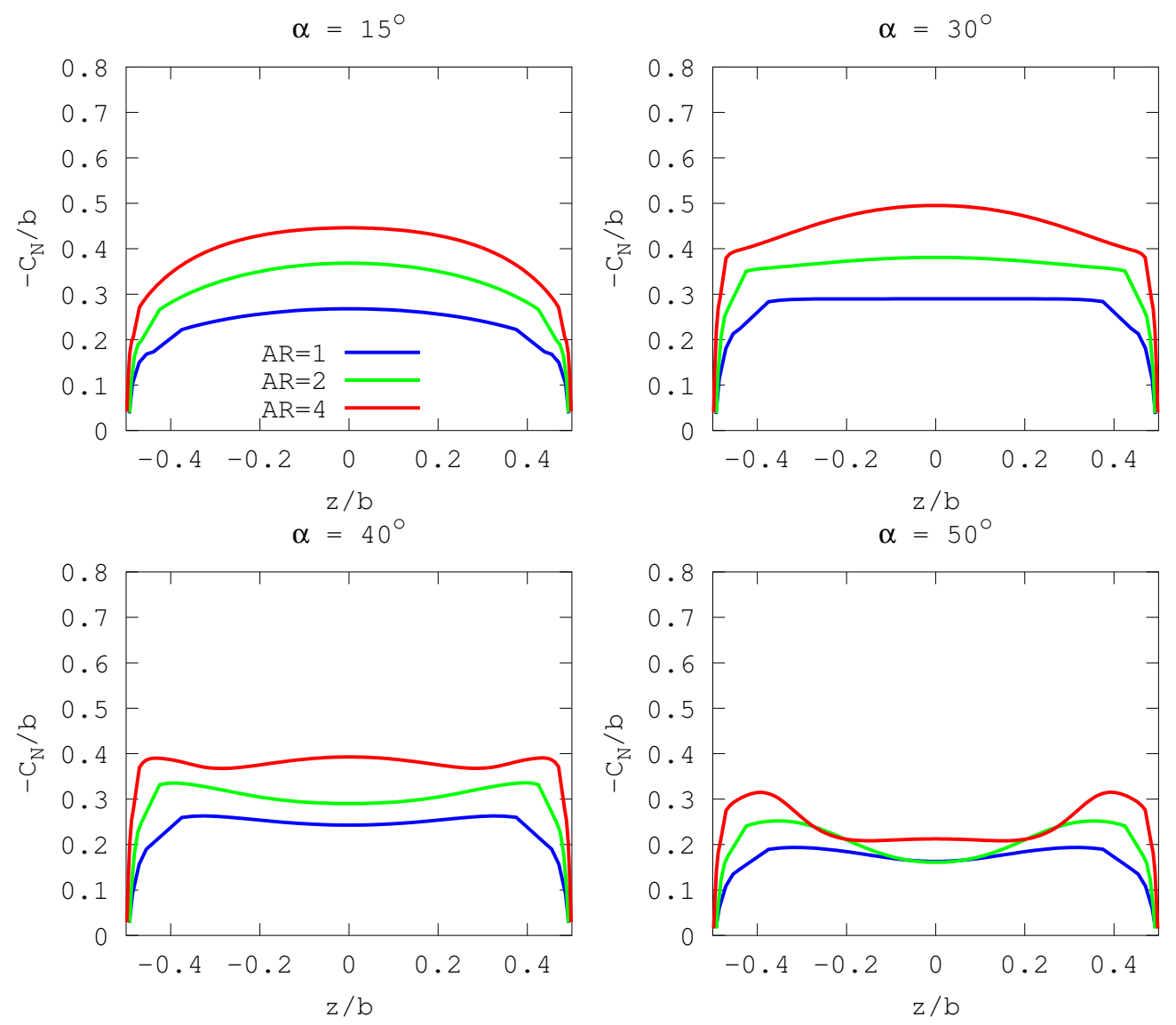

Figure 44: The normal force coefficient distribution plotted across the span of varying aspect ratios and angles of attack for the $\mathrm{K}=0.1$ perching.

From these force distributions, it was seen that the differences due arise in force generation between the pitch-up and perching motions and the pitch rates of $K=0.1$ and 0.2. The higher pitch rate developed a stronger LEV that lead to stronger forces acting on along the mid span. At the lower pitch rate, tip vortices were allowed to form, interfering with the LEV. Aspect ratio played a significant role also. The higher aspect ratio wings developed stronger mid span forces, while the lower aspect ratio wing had pronounced forces at the tips due to the tip vortices. The junction between the LEV and tip vortices saw a reduction in force production. Thus by limiting the growth of tip vortices, LEV force related production spans further across the wing. 
The 3D perching cases largely followed the same trends as the pitch-up cases. Higher aspect ratio plates developed stronger LEVs compared to those of lower aspect ratio. Tip vortex development was hampered by the pitching motion, and seem not able to move inward as easily when compared to the pitch-up cases. The addition of the flow deceleration (perching) only seemed to affect force magnitudes of the LEV and tip vortices. This inherently means both the LEV and tip vortices were less developed compared to their pitch-up counterparts.

\section{E. Pitching and Perching Conclusion}

The complexity of perch landing has led to a simplification of the kinematics involved into a pitch-up coupled with a deceleration in the streamwise direction. A parametric study has been conducted, varying the pitch, and deceleration rates of a varying aspect ratio flat plate at a Reynolds number of 500 to observe the how the aerodynamic forces vary on the plate in different cases.

The pitching maneuver was found to be Reynolds number sensitive which directly disagrees with the conclusions of Eldredge and Wang [45]. Peak lift was found to decrease with Reynolds number, while the noncirculatory forces added by the rotational acceleration at the beginning of the perching motion were found to be insensitive to Reynolds number.

Pitch-up numerical 2D, and 3D simulations produced similar trends when compared. The numerical 2D produced lower amplitude forces at a Reynolds number of 500, but had the same trends as observed as the experimental [8]. The 2D results were found to predict higher forces when compared to the 3D numerical predictions. This was contributed to effects of aspect ratio. As aspect ratio increased, it approached the $2 \mathrm{D}$ results and agreed with the $2 \mathrm{D}$ trend. Both showed if pitch rate was increased, there was a broadening and increase in the lift and drag coefficients.

Aspect ratio was also found to play a significant role in force production. As aspect ratio increased, maximum force generation was found to occur at lower angles 
Perching $\mathrm{K}=0.1$

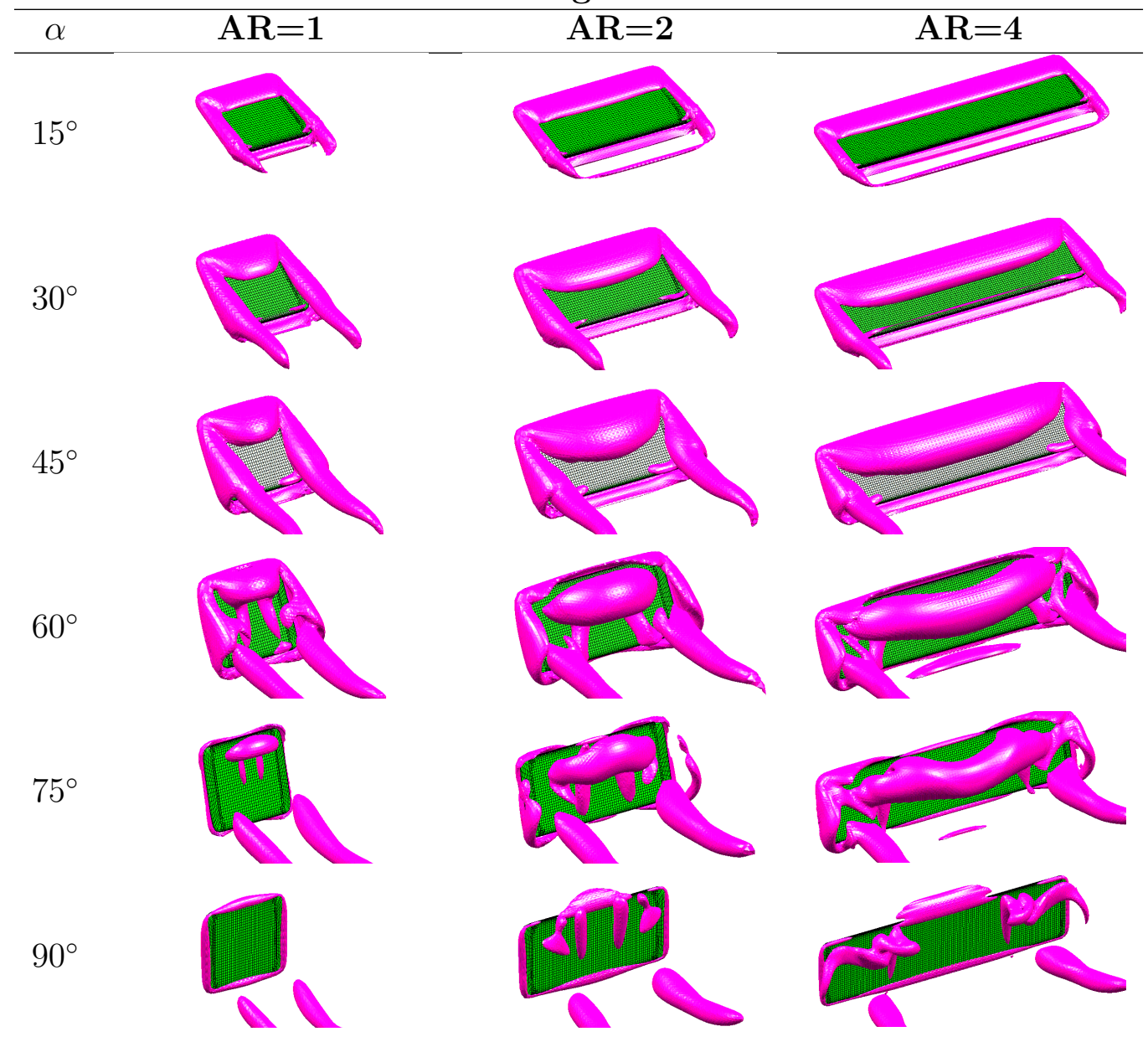

Figure 45: Iso-surfaces of the Q-criterion $(Q=1)$ at discrete angles of attack of each aspect ratio at $K=0.1$. Views are in the upstream direction looking at the trailing edge of the wing. 
Perching $\mathrm{K}=0.2$

(1)

Figure 46: Iso-surfaces of the Q-criterion $(Q=1)$ at discrete angles of attack of each aspect ratio at $K=0.2$. Views are in the upstream direction looking at the trailing edge of the wing. 
of attack. This is believed to be due to the changing flow structure on the suction of the plate as aspect ratio changes. The mid-span LEV vorticity was seen to increase as aspect ratio increased. Normal force distributions also showed that tip vortices affected less of the suction side of the plate as aspect ratio increased.

Changes in pitch rate resulted in similar effects as increased aspect ratio. As pitch rate increased, force generation also increased. Tip vortices were seen to affect the LEV produced force less, by having less time to develop due to increased pitch rate.

Finally, the perching motion shared the same conclusions of aspect ratio and pitching rate. The addition of flow deceleration resulted in reduced vortex size, and interior vorticity. However, the LEV remained closer to the wing at higher angles of attack, extending its influence on lift and drag. 


\section{CHAPTER VII}

\section{VORTEX CIRCULATION AND LIFT}

\section{A. Introduction}

Discussion thus far has shown that the LEV is affected by the Reynolds number, wing aspect ratio, and motion kinematics. To better our understanding of the complexity of the LEV, it is important to make quantitative analysis of the size and strength of the LEV. This in itself can be difficult, as the LEV cannot be measured directly like lift or drag forces.

Much work has gone into predicting the effects of the dynamic stall LEV via adaptations of panel code [72], potential flow models[17], and even empirically based models [73]. Ekaterinaris and Platzer offer an in-depth review of various computation dynamic stall prediction [18]. All these methods require a precise understanding of the flow around the airfoil, especially trajectories and strength of the LEV. However few to no studies have looked into understanding the LEV development across the suction side of the airfoil during the pitch motion. As pitch rate increases, the strength of the LEV increases, while the size decreases. However, the size of the LEV is also a function of viscosity. As viscosity increases (or a reduction in Reynolds number), the LEV becomes larger and more diffuse[25] as it was also seen in figure 27. While it is easy to understand why viscosity diffuses the LEV through examination the vorticity equation, little research exists attempting to understand how the LEV grows in size with pitch rate.

In recent years, several experimental studies have targeted the calculation of the LEV circulation strength by measuring and filtering the flow field about rotating, 
flapping, or pitching wings.[17, 74, 75, 76, 77, 78] Since the LEV circulation is related to the lift produced, calculating the LEV circulation can provide a uniquely defined value to understand how wing kinematics and geometry affect circulation. Recently, Baik et al. [76] experimentally studied the effect of reduced frequency on the development of the LEV circulation and LEV size of a nominally 2D pitch and plunge wing. They found that increasing the frequency could increase the LEV circulation strength but decrease the growth rate of the LEV which aligns with previous conclusions.

It is important to understand how the LEV develops across an airfoil. As an example, Granlund et al.[8] examined a perching wing, a variation of a dynamic stall for possible implications for landing maneuvers for MAVs, to better understand the flow phenomena involved. He found, as with several others, the LEV dominates the suction side of the airfoil for the majority of the motion. As previously stated, the presence of the large scale LEV, induces a large lift force on the airfoil [19]. Thus by understanding the LEV development, it is plausible that better control can be achieved for landing MAVs using the dynamic stall process to decelerate and maneuver itself.

The objective of this chapter is to numerically investigate the formation of the LEV and the change in LEV circulation during a pitch-up motion. In this study, the effects of pitch rate on the LEV circulation will be studied by eliciting a dynamic stall process with a single pitch-up motion. The LEV will be isolated using vortex identification techniques, and the circulation of the LEV will then be calculated. The circulation across the wing will be tracked throughout the entire pitching motion.

Thus the goals of this chapter are as follows: (1) To better understand how LEV forms with angle of attack at different pitch rates, (2) to draw a connection between LEV circulation strength and pitch rate, and finally (3) to quantify the lift contributed by the LEV to the pitching motion. The study will be conducted on a two dimensional computational grid at a Reynolds number of 500 along with various pitch rates at a reduced pitching motion of $0^{\circ}$ to $45^{\circ}$. These kinematics differ to decrease 
the computational cost of the simulation as a higher resolution grid was used for the analysis.

\section{B. Noncirculatory Force Contribution}

To be able to separate lift and drag due to circulation, the noncirculatory (NC) component of the lift coefficient, denoted as $C_{L, N C}$, must be separated from the total lift coefficient $\left(C_{L, M}\right)$ of the dynamic stall motion. Noncirculatory forces refer to the resultant pressure caused by the acceleration of fluid around the wing due to the wing's motion. [41] This can also be considered as the resistive force of the fluid pushing back on the wing as it moves. The noncirculatory force is known to be proportional to the pitch rate[8] and can contribute large portions to lift[77]. To effectively seperate the noncirculatory lift from the total lift, Theodorsen's model for flutting wings was used.

Theodorsens theory [79] is an analytical method for predicting the lift generated by fluttering wings with small pitch and plunge amplitudes. The theory is a summation of both circulatory and noncirculatory components of lift; however the circulatory component of the theory is only valid for low amplitudes of pitching and plunging wings. Ramesh et al. [80] found that the theoretical method for predicting the forces experienced by a pitching wing match remarkably well before the LEV separates from the wing. Examining Theodorsens equation, Eqn. 7.39, the coefficient

of lift generated by a fluttering wing is equal to the noncirculatory lift generated by the movement of the wing, the first set of terms, and the circulatory component due to the flow around the wing, the second set.

$$
\begin{gathered}
C_{L}=\pi b\left(-\frac{\ddot{h}}{U_{\infty}^{2}}+\frac{\dot{\alpha}}{U_{\infty}}-b a \frac{\ddot{\alpha}}{U_{\infty}^{2}}\right)+2 \pi C(k)\left[-\frac{\dot{h}}{U_{\infty}}+\alpha+b\left(\frac{1}{2}-a\right) \frac{\dot{\alpha}}{U_{\infty}}\right] \\
b=\frac{c}{2}
\end{gathered}
$$




$$
a=b\left(x_{p}-\frac{c}{2}\right)
$$

Where $\ddot{h}$ is the plunge acceleration, $x_{p}$ is the pivot location $(0.25 c), \dot{\alpha}$ and $\ddot{\alpha}$ are the rotational velocity and acceleration respectively, and $C(k)$ is known as Theodorsens function which is a complex number that brings in consideration the wake vorticity. Due to our interest only in the noncirculatory component, the entire second term on the right side of Eqn. 7.39 is dropped. A second simplification can be made by dropping the $\ddot{h}$ terms since there is no plunge motion in the studied pitchup kinematics. This further reduces the Theodorsen model for the noncirculatory lift $\left(C_{L, N C}\right)$ to a simple function that only relies on free stream velocity, and pitch kinematics.

$$
C_{L, N C}=\pi b\left(\frac{\dot{\alpha}}{U_{\infty}}-b a \frac{\ddot{\alpha}}{U_{\infty}^{2}}\right)
$$

A final adjustment must be made since the original Theodorsen model was derived for small changes in angle of attack. The wing in this study pitches to a maximum of 45 degrees where small angle assumptions no longer apply. Thus Eqn. 7.42 would actual refer to the magnitude of the normal force coefficient, $C_{F, N C}$, acting on the wing. For the large angle of attack, the real $C_{L, N C}$ can be found by multiplying $C_{F, N C}$ by the cosine of the instantaneous angle of attack.

$$
\begin{gathered}
C_{F, N C}=\pi b\left(\frac{\dot{\alpha}}{U_{\infty}}-b a \frac{\ddot{\alpha}}{U_{\infty}^{2}}\right) \\
C_{L, N C}=C_{F, N C} \cos (\alpha)
\end{gathered}
$$

From these sets of equations, it is now possible to separate the lift due to circulation $\left(C_{L, C}\right)$ from the total lift coefficient $\left(C_{L, M}\right)$ by continuing with the assumption that noncirculatory and circulatory lift are linearly additive with the following equation: 


$$
C_{L, C}=C_{L, M}-C_{L, N C}
$$

\section{Circulatory Lift Contribution}

Previously, the noncirculatory lift was found using Theodorsen's equation, to find the circulatory lift component of total lift. The circulatory component of the Theodorsen equation was dropped due to interest only in the noncirculatory component. Since $C(k)$ is also an unknown in the equation and cannot be easily calculated for this simulation, the circulatory lift is calculated by examining the LEV circulation strength.

The circulatory lift $\left(C_{L, C}\right)$ contribution is handled by equating it to be proportional to the circulation strength of the $\operatorname{LEV}\left(\Gamma_{L E V}\right)$. The circulation of the LEV was found by integrating the clockwise vorticity $\left(\omega_{z, C W}\right)$ that was identified to be inside of a vortex via the Q-Criterion $(Q>0)$ hence Eqn. 7.46. This allows for the contribution of lift by the $\operatorname{LEV}\left(C_{\Gamma, C}\right)$, Eqn. 7.47, to be calculated. Note the addition of a cosine in Eqn. 7.47 is to correct for small angle assumptions.

$$
\begin{aligned}
\Gamma_{L E V} & =\iint_{Q>0} \omega_{z, C W} d A \\
C_{L, \Gamma} & =\frac{2 \Gamma_{L E V}}{U_{\infty} c} \cos (\alpha)
\end{aligned}
$$

The z-axis vorticit $\left(\omega_{z}\right)$ is prefered in this chapter (and future) as the LEV rotates predominately around the z-axis. Vorticity for $3 \mathrm{D}$ wings that does not rotate about the z-axis can be difficult to track as their orientation can change. Thus only the z-axis vorticity is analysized.

Due to the noncirculatory effects of the pitching motion, $C_{L, L E V}$ will not accurately depict the lift induced by the LEV. The pitching motion itself will induce circulation around the airfoil. Thus the noncirculatory portion of the lift must be 
separated again from the newly calculated LEV lift circulation. This yields a second equation that describes the circulatory lift of the pitching airfoil due to the presence of a LEV.

$$
C_{L, C}=C_{L, \Gamma}-C_{L, N C}
$$

If Eqn. 7.45 were substituted in Eqn. 7.48, it would yield that $C_{L, \Gamma}$ is either equal or approximately equal to $C_{L, M}$. Equations 7.45 and 7.48 should both predict the same circulatory lift contribution due to the LEV; however Eqn. 7.47 is only valid until stall where the LEV sheds from the airfoil.

\section{Circulatory and Noncirculatory Forces}

Figure 4 compared the variation of angle of attack with time using different values of the smoothing parameter $a$ for $K=0.1$, and $K=0.2$ for comparison. As $a$ increases, the pitch-up motion transits from a smoothed ramp to a sharp ramp function. The effect of $a$ appears benign on the angle of attack profile; however the effects are apparent with angular velocities and accelerations. With the increase in $a$, angular velocity and acceleration magnitudes increase at the beginning of the motions. This would in turn leads to higher noncirculatory forces during the non-zero

acceleration phase as described in Eqn. 7.42. Granlund et al. [81] found that a does not change the aerodynamic forces during the constant angular velocity portion of the motion.

Noncirculatory forces can influence the magnitude of the forces measured depending on the pitch-up profiled used. Figure 4 illustrated how the smoothing variable $a$, and $K$ impacted the angular velocity and acceleration. Noncirculatory forces are a function of these two quantities. As $K$ increases, noncirculatory forces also increase. This was also seen in Granlund et al.[24]. The effect is most visible in the $C_{L}$ plots between $0^{\circ}$ and $5^{\circ}$ where the rotational acceleration is nonzero shown later in figures 28. 


\section{Noncirculatory Component}

First looking at the noncirculatory component of lift $\left(C_{L, N C}\right)$, figure 47 (a) plots the calculated coefficient of lift $\left(C_{L, M}\right)$, and noncirculatory lift coefficient calculated from Eqn. 7.42. It can be seen that the noncirculatory component does make up a significant portion of the sum lift generated for all pitch rates: $16.8 \%, 21.8 \%$, and $23.5 \%$ of the lift for the respective cases of $0.05,0.1$ and 0.2 . As the pitch rate increases, so does the amount of noncirculatory contribution. As it can be seen, the noncirculatory lift is only a function of the kinematic motion. Thus changing pitch rate only increases or decreases the magnitude.

By subtracting out the noncirculatory component, Eqn. 7.48, the resulting lift profiles, 47(b), for the tested pitch rates collapse onto one another eliminating their offset from one another. This further confirms that the noncirculatory component is linearly additive to the circulatory component of the generated lift as was shown in the Theodorsen model, Eqn. 7.39. The resulting lift can be assumed to the resultant circulatory component from the LEV.

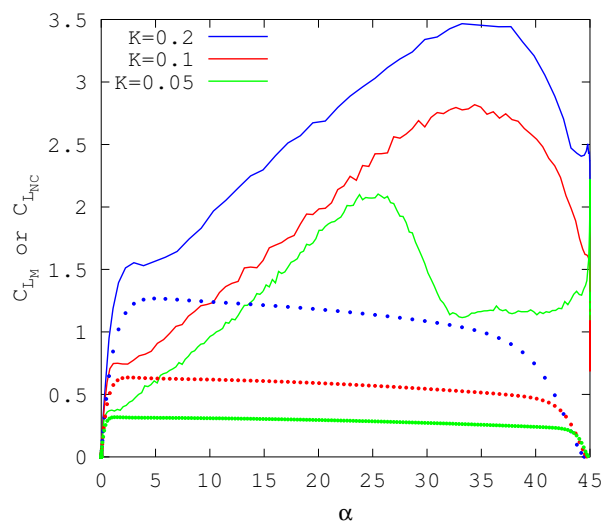

(a)

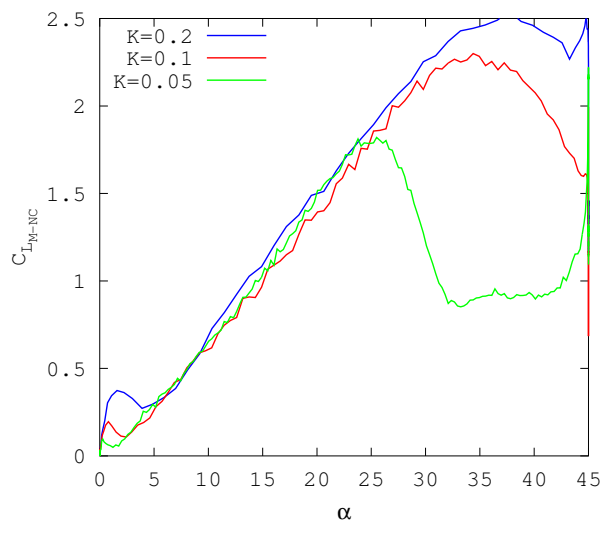

(b)

Figure 47: (a) Lift (solid) and noncirculatory (dotted) versus angle of attack of three pitch rates. (b) Resultant lift coefficient with noncirculatory component removed. 


\section{Circulatory Component}

The circulation induced by the presence of the LEV was measured using the previously discussed filtering and vorticity integration techniques and is reported in 486(a) The circulation lift, $C_{L, \Gamma}$, is adjusted for the direction of lift using Eqn. 7.47 and compared directly to the measured lift coefficient.

For the $\mathrm{K}=0.2$ case, Eqn. 7.47 predicts the lift coefficient well up to the point of stall at 30 degrees, past this the two begin to diverge. The same trend is not seen for the two slower pitch rates where the circulation diverges from the actual lift coefficient at approximately a third of their stall angle. This may be due to the LEV growing in size and convecting away from the surface of the plate, resulting in a reduced lift coefficient. Without taking in account the position of the LEV to the plates surface, it may be difficult to predict the actual lift by solely calculating the circulation strength of the LEV.

Much like the previous section, the noncirculatory component can be separated out of the circulation lift (Eqn. 7.45 and 7.48), also shown in figure 48(b) Again, the circulation for each pitch rate collapses onto a collective trend. This again confirms the linearly additive nature of noncirculatory and circulatory forces; however, without a proper description of how the LEV convects away from the plate to correlate the lift force, results using the circulation are only valid to approximate lift at low angles of attack. Figure 48(b) does not display any inherently obvious relationships of growth in circulation to pitch rate. It was found if the circulation calculated from Eqn. 7.48 was normalized by pitch rate; the trends would collectively collapse on one another. Figure 49 shows that the lift due to circulation can be correlated to pitch rate and

the nondimensionalized flow sweeps across the plate. Each pitch rate follows the same trend until stall occurs, which then they quickly plateau and then diminish. This helps clarify how lift due to circulation changes with pitch rate, it is directly proportional to pitch rate. Where circulation develops along a prescribed path that is a function of the pitch rate. 
Understanding how pitch rate affects circulation is a crucial element if one wishes to predict or understand how a pitching wing will produce lift. Figure 49 shows that the circulation produced is only depended on the time and the pitch rate. This effectively gives a relationship as to how specific circulation forces (lift) can be achieved at what pitch rate, and how long it will take.

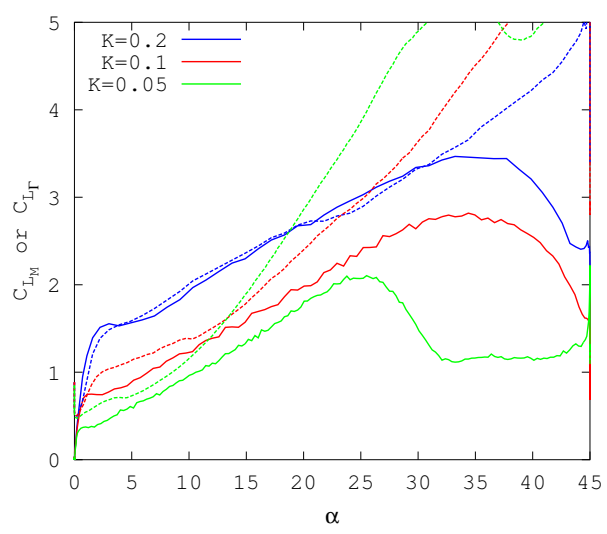

(a)

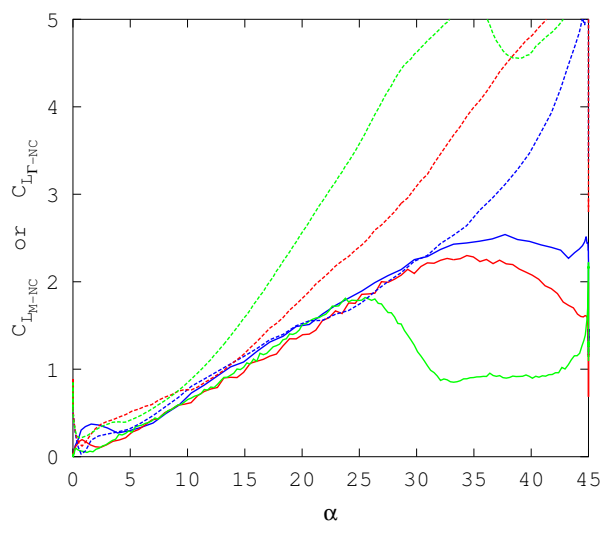

(b)

Figure 48: (a) Actual lift (solid) and circulatory (dotted) coefficients versus angle of attack of three pitch rates. (b) Actual and circulation lift coefficients with noncirculatory component removed.

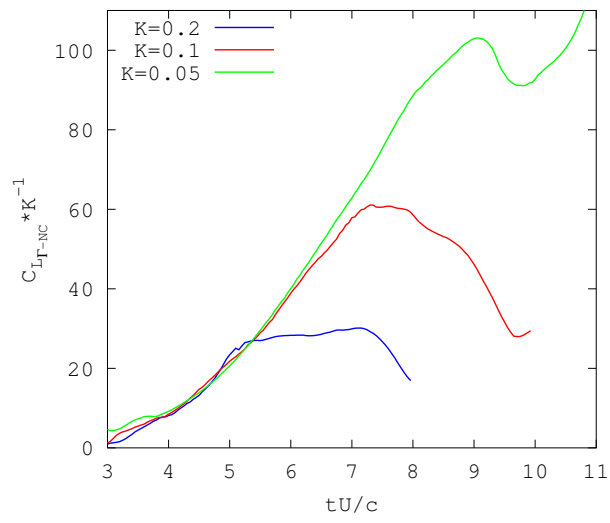

Figure 49: Coefficient of lift due to circulation divided by pitch rate versus the nondimensionalized time since the start of the pitch-up motion. 


\section{E. 2D Vortex Size}

A first pass approximation was used when measuring the radius of the vortex. Using the vorticity filtering technique previously described, the area occupied by the LEV was measured. Simply put, any area that was positively identified as a vortex was assigned with a value of 1 , and areas that were not, 0 . The domain was integrated again to find the area occupied by the vortex. It was simply assumed that the LEV was a perfect circle, and the radius of the LEV could be back calculated from the measured area as shown in figure 50. Any other clockwise vorticity that made it through the filter was assumed to be part of the LEV.

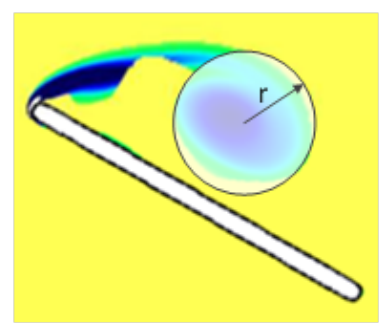

Figure 50: The area occupied by the vortex is assumed to be a perfect circle

The vortex size was calculated throughout the entire pitch- up duration of each pitch rate and is shown in figure 51(a). All three pitch rates have similar early vortex growth rates; however, all three at the same angle attack quickly diverge from one another. This is due to the LEV beginning to roll up at a specific angle of attack between angles of 15-20 degrees. Passed this angle it was seen that the lowest pitch rate had a higher LEV growth rate, than the higher pitch rate. For all pitch rates, the LEV growth is constant passed the angle of 15 until stall. The lowest pitch rate shows a deep plunge in vortex size at an angle of 30 degrees. This is due to the shed LEV quickly dissipating downstream.

Examining the difference in slope of each pitch rate it is important to remember, that the pitch rate of $\mathrm{K}=0.05$ has twice the time to develop a LEV when compared to $\mathrm{K}=0.1$, and four times when compared to $\mathrm{K}=0.2$, thus the lower pitch 
rate will have a larger vortex radius at higher angles. This suggests that the LEV growth rate is not a function of angle, but a function of time. To illustrate this, three separate snapshots were taken of the pitching plates when the LEV radius was equal to 0.2 . Figure 52 shows how even at separate pitch rates, the LEV is of comparable size and shape. The primary differences seen between the three cases are the location and strength of the LEV. For the slower pitch rate of $\mathrm{K}=0.05$, the LEV has had more time to propagate downstream, and diffuse resulting in a weaker contour. The lowest pitch rate achieves the vortex radius at a lower angle than the higher pitch rates. This is due to the vortex growing at a specific rate that is time dependent. Thus the plate is simply rotating under the vortex while it grows at its own pace. Thus the LEV forms at later angles of attack at higher pitch rates simply due to the fact it takes a prescribed amount of time for them to form.

Interestingly, by plotting the individual pitch rates of the calculated circulation lift coefficient, Eqn. 7.47, and vortex radius, this is shown (Figure 51(b)), it can be seen that with increasing pitch rate, the line slope increases. At a given circulation coefficient, it can be seen with higher pitch rates produce a smaller vortex. While this has always been observed in previous works $[18,16,21,22,20,8]$, it is rarely quantified. This also shows how the circulation strength of the LEV is less of a function of radius, but more dependent on pitch rate. 


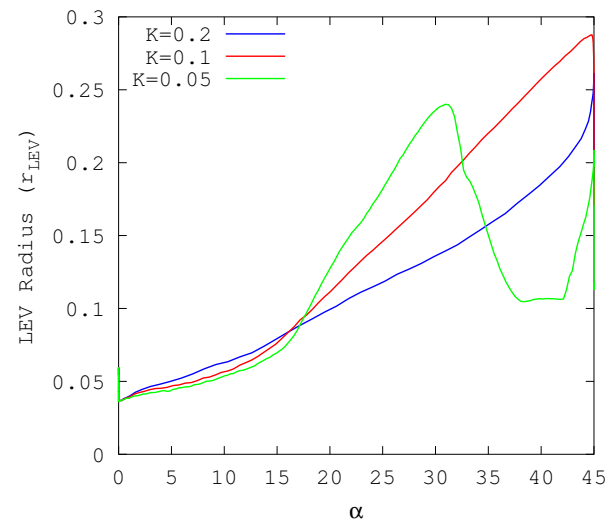

(a)

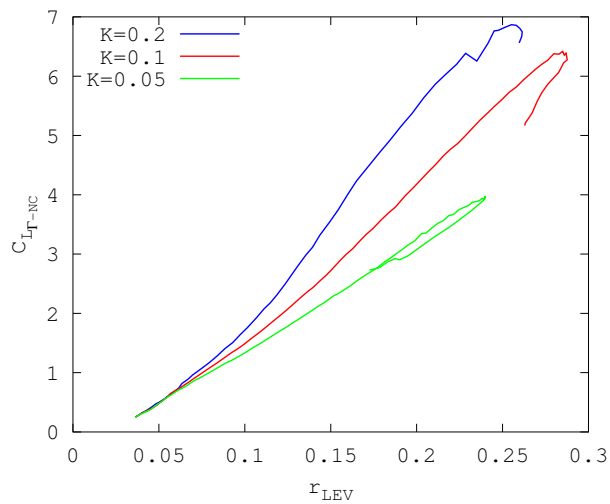

(b)

Figure 51: (a) Radius of LEV versus angle of attack of three pitch rates. (b) Radius of LEV versus circulation lift coefficient.
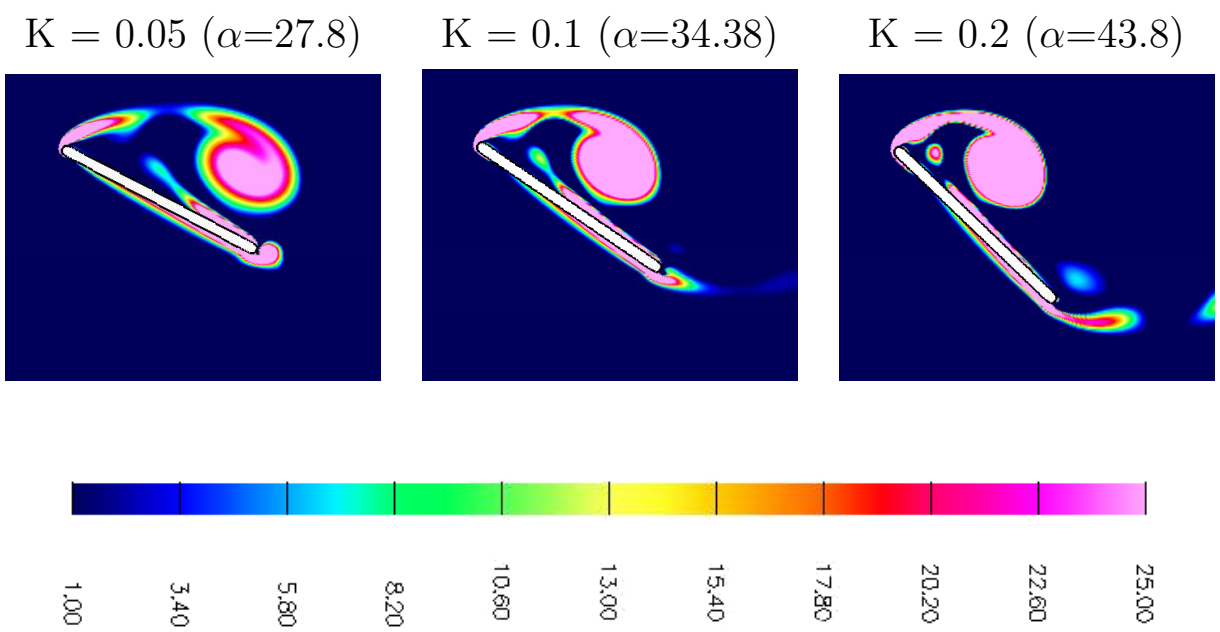

Figure 52: Snapshots of the Q-Criterion $(1<\mathrm{Q}<25)$ when $r_{L E V}=0.2$ at three different pitch rates.

\section{F. Circulation and Lift Conclusion}

The examination of the lift contribution of the LEV has been conducted. Modeled after the Theodorsen method, the lift coefficient produced by a pitching plate was investigated by examining the noncirculatory and circulatory forces. It was found 
that the noncirculatory component of the measured lift force could be separated out, leaving only the force produced by any circulatory effects. Results have shown that noncirculatory forces only contribute $10-20 \%$ of the lifting force and the remaining is due to the LEV.

The lift produced by the LEV was also found to match well at low angles of attack; however as angle of attack increased, the lift produced by the LEV quickly diverged from the calculated lift coefficient. Thus any experiment measuring lift via vorticity in the domain, must take in account how the LEV propagates away from the airfoil to correlate correct lift coefficient. Otherwise, the lift found would greatly overestimate the actual lift on the wing.

By comparing vortex size and circulatory lift together, a tangible trend was produced that shows how pitch rate effects circulation strength and vortex size. While observed in other works, but never quantified, it was shown that higher pitch rates produce smaller LEVs with higher circulation strength. The vortices are primarily smaller due to a time constant of vortex formation. Higher pitch rates pitch faster than the vortex can form; however only increases the vortex circulation strength and not the radius. The increase in vortex radius is from the diffusion of the vortex through viscous effects. Thus higher pitch rates produces a smaller yet stronger LEV by pitching faster than it can grow. This effectively means that the strength of the LEV is a function of pitch rate (or time), not angle of attack. 


\section{CHAPTER VIII}

\section{GEOMETRY AND KINEMATIC EFFECTS ON CIRCULATION}

\section{A. Introduction}

In the previous chapter, the effects of pitch rate on a $2 \mathrm{D} \mathrm{LEV}$ was investigated on a lower angle of attack pitching wing. The previous chapter will be re-evaluated for the pitching and perching kinematics for the 3D wings; however the separation of noncirculatory forces will not be performed. The previous theory presented is only valid for a $2 \mathrm{D}$ analysis, and not a 3D. Therefore in this chapter, the vortex circulation will be investigated as the summation of both types of circulation.

For the 3D analysis, the circulation will be investigated in three ways: mid-

span, total, and span distribution. Similar to the 2D analysis, the circulation due to the LEV will be calculated at a center cross-section of each tested plate, this is then expanded to include the entire span circulation. Spanwise circulation is presented by calculating the LEV circulation at individual slices around the wing span. This provides a detailed contour map of how the circulation changes with angle and pitch rate across the wing.

\section{B. Mid and Quarter Span Circulation}

\section{Pitching}

Before examining the LEV circulation variation across the wing span, the circulation due to LEV and shear layer vortices is evaluated. The LEV circulation was 
calculated using Eqn. 7.46 with $Q>0$ while the circulation from shear layer vortices was calculated with $Q<0$. The total circulation was calculated by removing the Q-criterion filter. The approach is similar to that of the 2D pitching analysis, with the exception of evaluating Eqn. 7.46 at finite locations across the span. This approach provides a simplistic view of the development of the total, LEV, and shear layer circulation with angle of attack. Figure 53 and 54 plots the three calculated circulation values $\left(\Gamma_{C W}\right)$ at the quarter and mid-span locations of the three aspect ratios at $K=0.1$ and $K=0.2$.
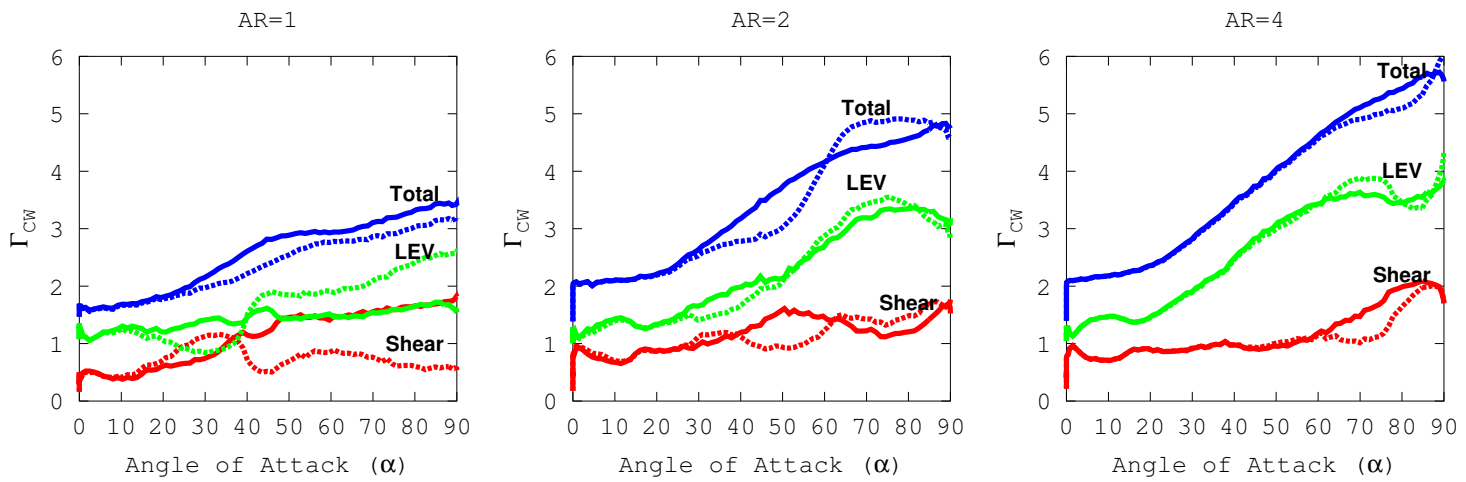

Figure 53: Total, LEV, and shear quarter and mid-span circulation for each aspect ratio for $K=0.1$. Dotted and solid lines represent $z / b=0.25$ and $z / b=0.5$ respectively.

As a general trend for both values of $K$, as the aspect ratio increases, so does the LEV circulation, and shear circulation remains stagnant. The $A R=4$ plate has a near uniform circulation distribution throughout the entire pitching cycle when comparing mid and quarter span. $A R=2$ has a less uniform distribution, but circulation at the two locations follow the same trend. For the lowest tested $A R$, the shear and LEV circulation are inversely proportional to one another. Mid pitch, shear switches to LEV related circulation. Again this is seen for both pitch rates. Due to the small aspect ratio, tip-vortices effect the flow development over the wing. As the wing pitches up, tip vortices grow in size, and damp LEV development, resulting in 

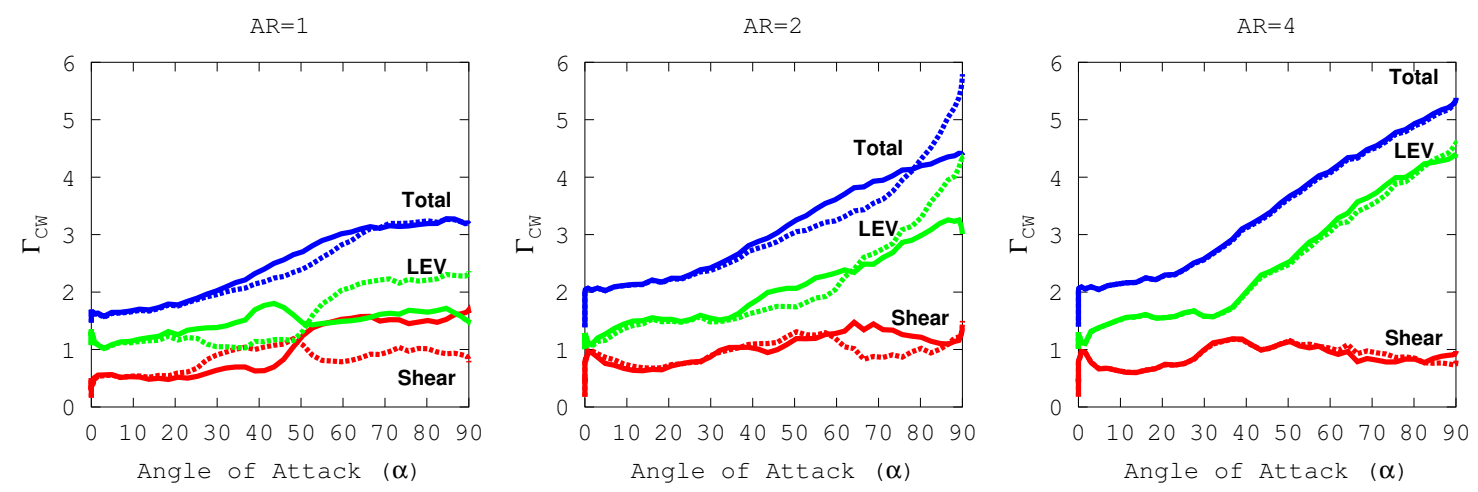

Figure 54: Total, LEV, and shear quarter and mid-span circulation for each aspect ratio for $K=0.2$. Dotted and solid lines represent $z / b=0.25$ and $z / b=0.5$ respectively.

rapidly changing vorticity alignment.

An examination of the vorticity transport equation (Eqn. 8.49), the first two term on the right hand side describes that vorticity can be stretched or tilted. Thus any LEV or $\omega_{z}$ vorticity can be titled into other planes from interaction with tip vortices. This also explains the inversely proportional nature of the LEV and shear circulation seen in figure $54(A R=1)$ at $50^{\circ} . \omega_{z}$ vorticity is being modified (tilted) by tip vortices in and out of the $z$ plane used to identify vortices. Because of this complex LEV and tip vortex interaction, lift producing vorticity may exist in several planes that is not bound to the strength of the LEV. Thus this attenuates aerodynamic forces on the $A R=1$ wing since with lift producing vorticity does not occur in the $\omega_{z}$ form which would shed with the LEV at stall.

$$
\frac{D \vec{\omega}}{D t}=(\omega \cdot \nabla \vec{u})-\omega(\nabla \cdot \vec{u})+v \nabla^{2} \vec{\omega}
$$

By integrating the circulation across the span, the total generated circulation can be compared. This accounts for changes in flow structure due to aspect ratio. This was done by integrating Eqn. 7.46 along the span of the wing, and the results are shown in figure 55. $A R=2,4$ show a diverging LEV and total circulation with an increase in $K$. Initially, $\Gamma_{C W}$ for both $K$ values trend together up to approximately 
$25^{\circ}$. The $K=0.2$ lines remain stagnant until $35^{\circ}$, which then increase at a similar rate as $K=0.1$. This suggests that the timing of circulation development across the wing is a function of $K$.
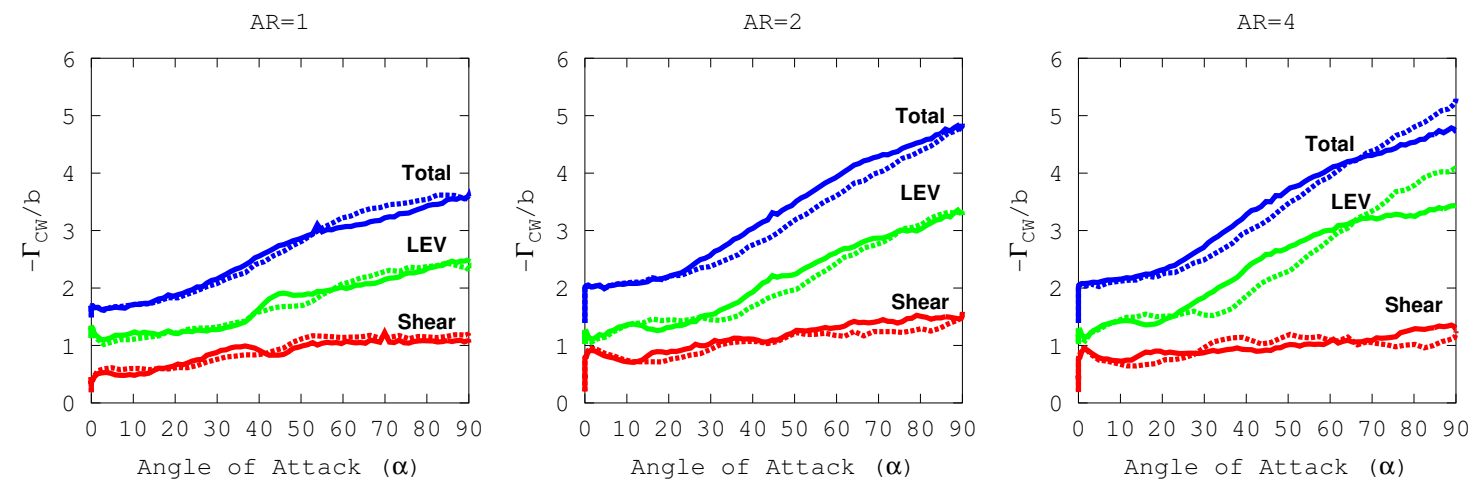

Figure 55: Total, LEV, and shear circulation across the entire span for each aspect ratio and $K$. Solid and dotted lines represent $K=0.1$ and 0.2 respectively.

The effect of $K$ on the $A R=1$ wing is less clear than the larger $A R$ wings. The LEV, shear layer, and total circulation show little variation with the reduce frequency $K$. As previously shown in figure 35, the mid-span LEV vorticity is smaller resulting in lower calculated $\Gamma_{C W}$. Again the tip vortices that from on the $A R=1$ wing occupy a significant portion of the suction side surface. (This is shown later in figure 59.) This would result in circulation that is not captured by the $\omega_{z}$ component.

\section{Perching}

For the perching cases, the mid and quarter span circulation calculations are plotted in figure 56 and 57 for the $K=0.1$ and 0.2 rates respectively, and the total span circulation is in figure 57. For each aspect ratio, the same increase in circulation is seen as the aspect ratio increases. Differences in the mid and quarter span circulation plots are also seen to reduce as aspect ratio increases. Unlike the pitching motion, circulation either decays with the progression of the perching maneuver, or varies little with angle. 

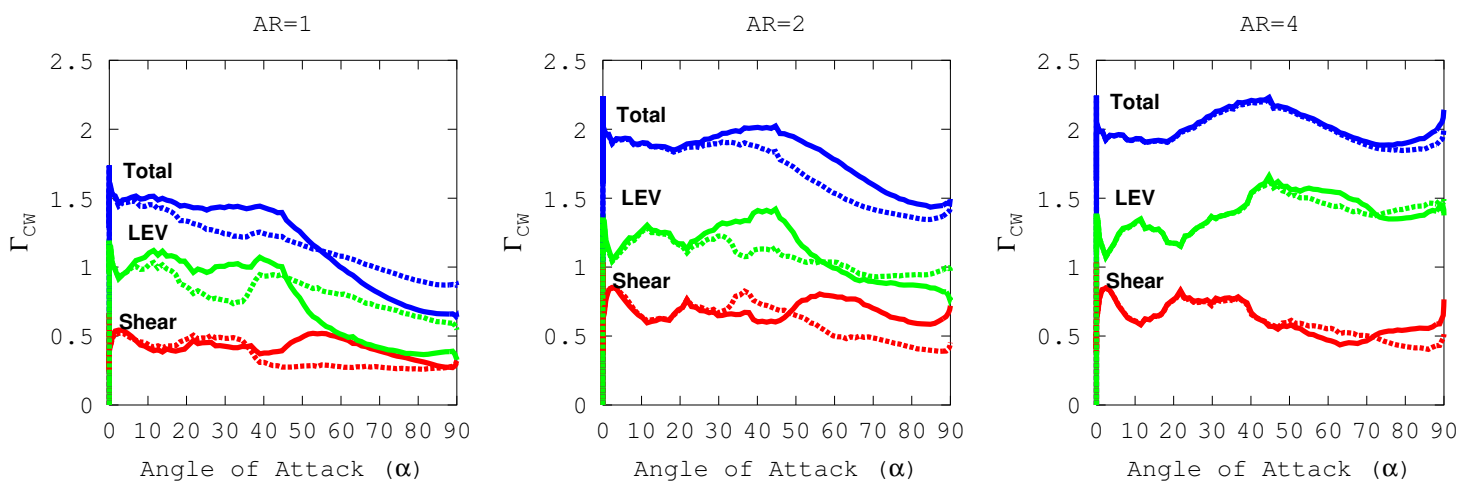

Figure 56: Total, LEV, and shear quarter and mid-span circulation for each aspect ratio for $K=0.1$. Dotted and solid lines represent $z / b=0.25$ and $z / b=0.5$ respectively.
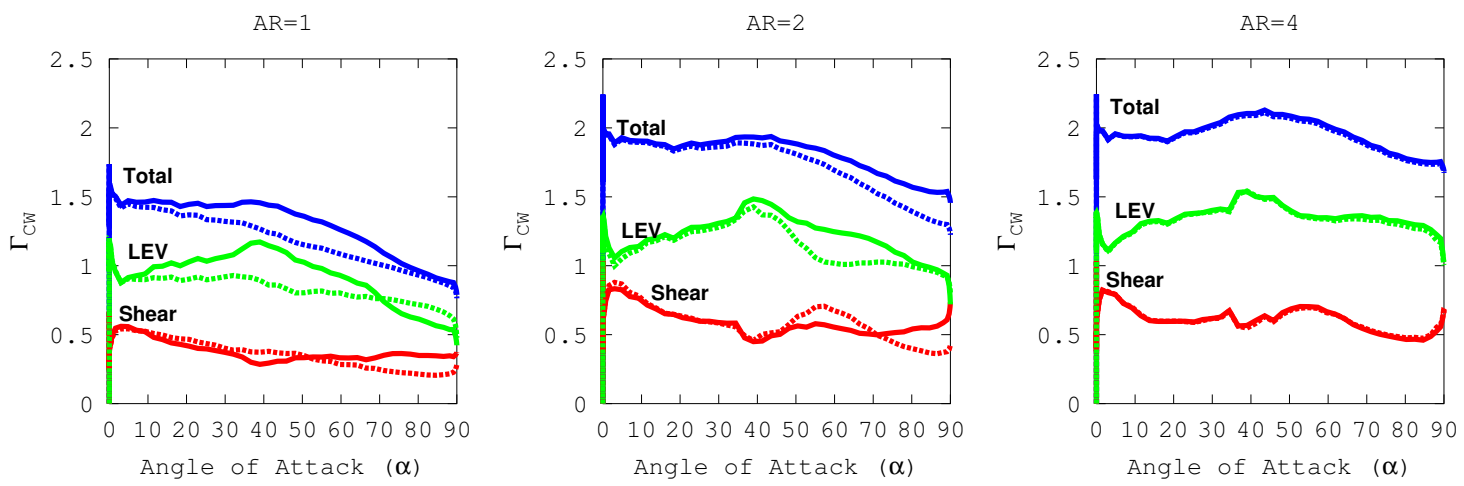

Figure 57: Total, LEV, and shear quarter and mid-span circulation for each aspect ratio for $K=0.2$. Dotted and solid lines represent $z / b=0.25$ and $z / b=0.5$ respectively.

The first is seen with the $A R=1$ wing. The circulation established at the start of the motion decays as the wing pitches and decelerates. Again as discussed in the pitching section, the mid-span LEV vorticity is lower resulting in a lower $\Gamma_{C W}$. This weakened LEV is further dissipated by the flow deceleration.

The $A R=4$ wing illustrates the effects of the captured LEV that was seen in figure 36. The larger aspect ratio allowed the establishment of a LEV even with flow 
deceleration. As the motion continues, less flow is available to maintain or feed the vortex resulting in a decelerated decay rate of the LEV.

Examining the total circulation across the span, pitch rate has a benign effect on the circulation history. Primarily, increased pitch rate increases the peak LEV circulation. For both pitch rates, this occurs approximately at $40^{\circ}$.
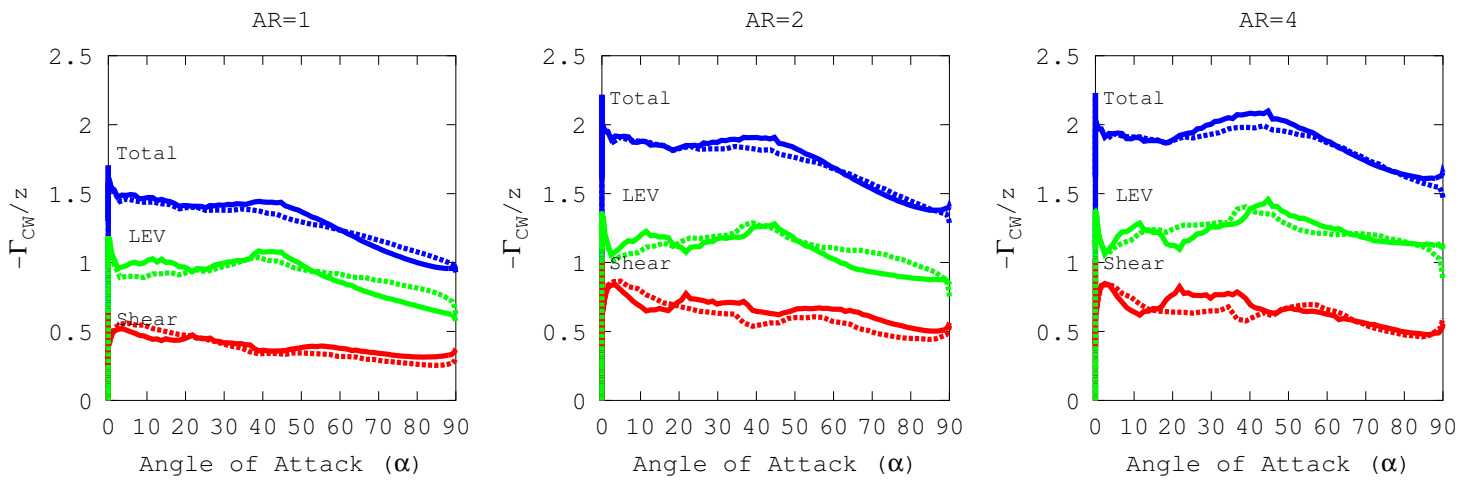

Figure 58: Total, LEV, and shear circulation across the entire span for each aspect ratio and $K$. Solid and dotted lines represent $K=0.1$ and 0.2 respectively.

\section{Spanwise LEV Circulation}

\section{Pitching}

Figures 59, 60, and 61 plot the resultant $\Gamma_{L E V}$ contours from all tested cases. It is important to stress that these contours are not $\Gamma_{L E V}$ distributions across the entire surface of the wing at a single angle of attack, but rather they are the variation in $\Gamma_{L E V}$ across the normalized span $(z / b)$ through the entire pitching motion. Due to wing symmetry only half wing is shown. Each contour plot shows $K$ value of 0.1 and 0.2 along side iso-surface plots of $Q=5$ at three angles of attack are shown for both $K$ values. 


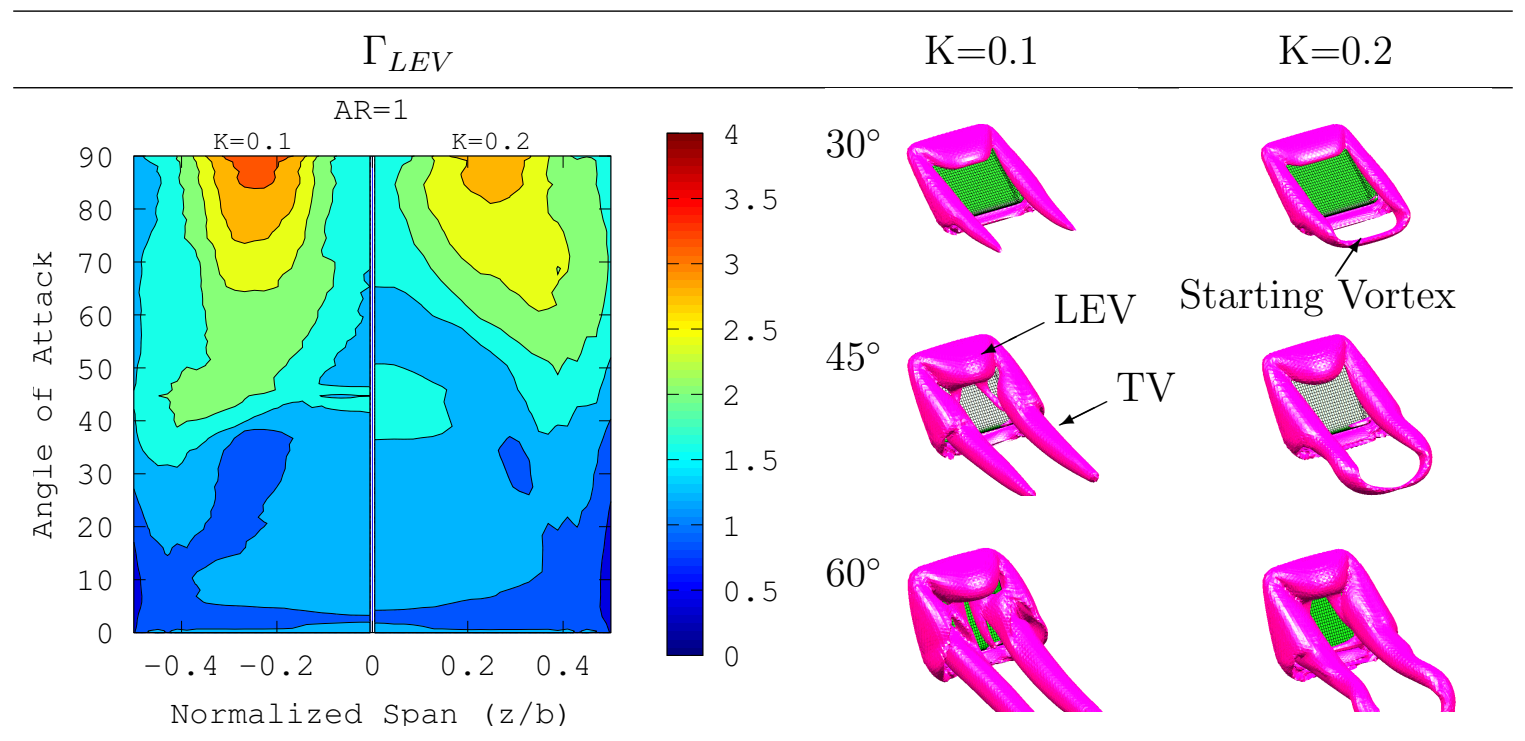

Figure 59: (Left) Contour of spanwise $\Gamma_{L E V}$ through entire pitching motion for the $A R=1$ wing at both tested $K$ values. (Right) Iso-surface contours where the $Q=5$ for both $K$ values at $30^{\circ}, 45^{\circ}$, and $60^{\circ}$

Beginning with figure 59, the $A R=1$ wing, the $Q$ iso-surfaces of both $K$ cases show similar vortex structures. The starting vortex is still visible in the $K=0.2$ case, while it has already convected away from the plate in the $K=0.1$ case. When wings are pitched to the same angle of attack, twice the amount of time has elapsed for the $K=0.1$ case compared to the $K=0.2$ case. The lower pitch rate wing allows the LEV to have more time to develop and also allows tip vortices to grow larger and interfere with the LEV. This phenomenon was also seen in the experiment of Ol et al. [30]. However, it is difficult to identify from the $\Gamma_{L E V}$ contour any spanwise LEV circulation for either $K$ value even though a LEV-like structure appears above the wing. Several authors have commented on how tip vortices can affect LEV development. Jones and Babinsky [74], and Coton and Galbraith [36] found that the tip vortices impeded LEV development on low aspect ratio wings $(A R<2)$. At 
low aspect ratios, tip vortices occupy a large portion of the wing, which disrupts the development of spanwise $\omega_{z}$ related circulation. The lack of LEV circulation would suggest a lack of or significant decrease in lift production; however as previously mentioned, the lift inducing circulation may not be tracked by $\omega_{z}$.

Examining figure 60 for the $A R=2$ wing reveals the difference in circulation development with an increase in aspect ratio. It shows that the reduced pitch rate has an effect on the development of circulation, which was not apparent for the $A R=1$ wing. Examining the $K=0.2$ contours shows circulation development $\left(\Gamma_{L E V}>1.5\right)$ at a higher angle of attack when compared to $K=0.1$. This delay in development was also seen in figure 55. Increasing $K$ was also seen to delay the formation of tip vortices. This is evident in the contour plots. Towards the tips, circulation quickly drops in the lower $K$ case. By delaying the tip vortex formation, the LEV circulation occupied more of the span. This lead to a larger area of LEV circulation covering the span. 


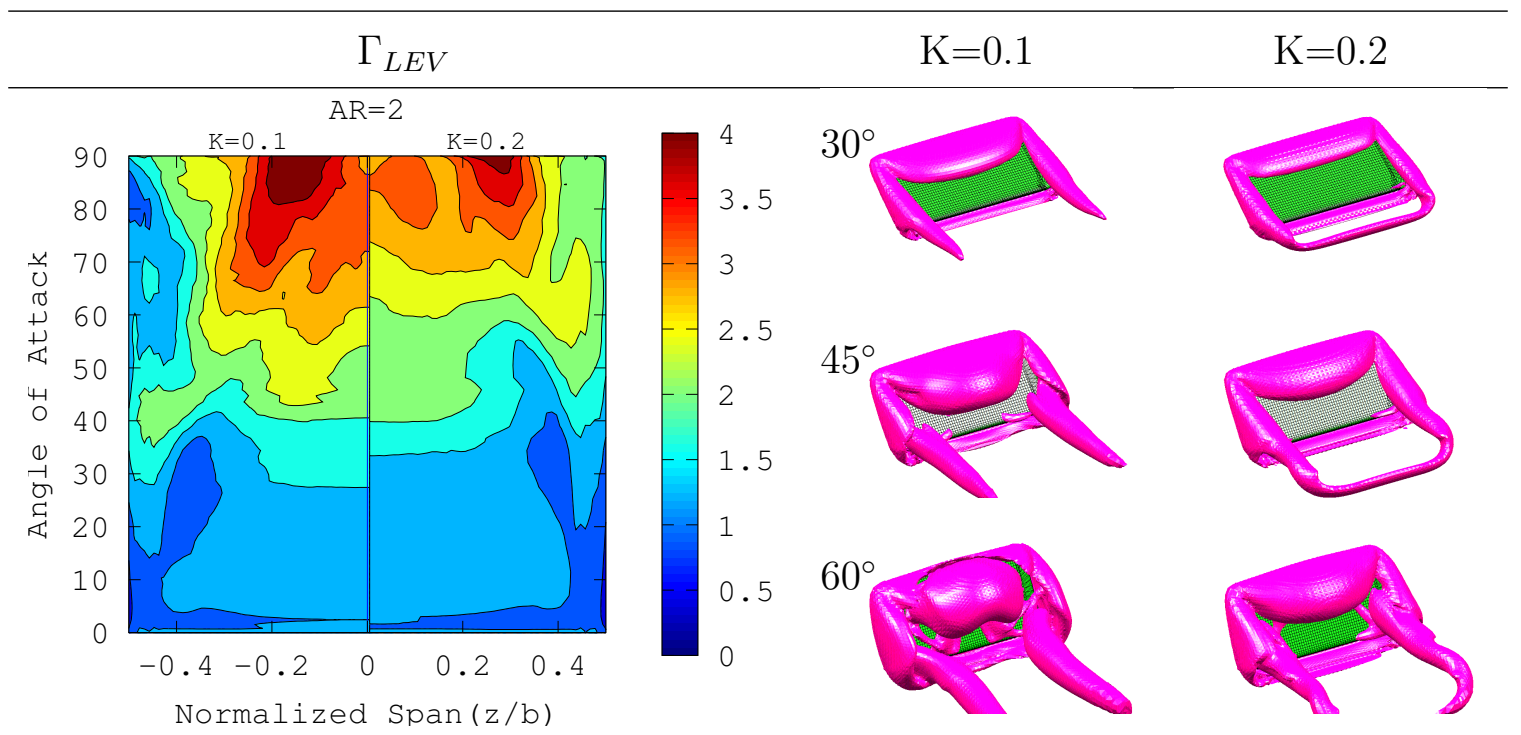

Figure 60: (Left) Contour of spanwise $\Gamma_{L E V}$ through entire pitching motion for the $A R=2$ wing at both tested $K$ values. (Right) Iso-surface contours where the $Q=5$ for both $K$ values at $30^{\circ}, 45^{\circ}$, and $60^{\circ}$

The $A R=4$ plots (figure 61 ) share many similarities with the $A R=2$ plots: later circulation development and a reduction in LEV and tip vortex interaction with increased $K$. Examining the contour plots shows that the circulation develops later in the pitching motion and it is more uniform across the span compared to the lower $A R$ wings. Again the circulation is seen to drop towards the edge of the spans. In the $K=0.1$ case, tip vortices push the circulation inward by approximately $5 \%$ and even less for $K=0.2$. The $A R=2, K=0.1$ case showed deeper inward push of $10 \%$ of circulation by tip vortices. Geometric scaling (increased span) of the plate would explain the decrease of inward of the circulation by the tip vortices. 


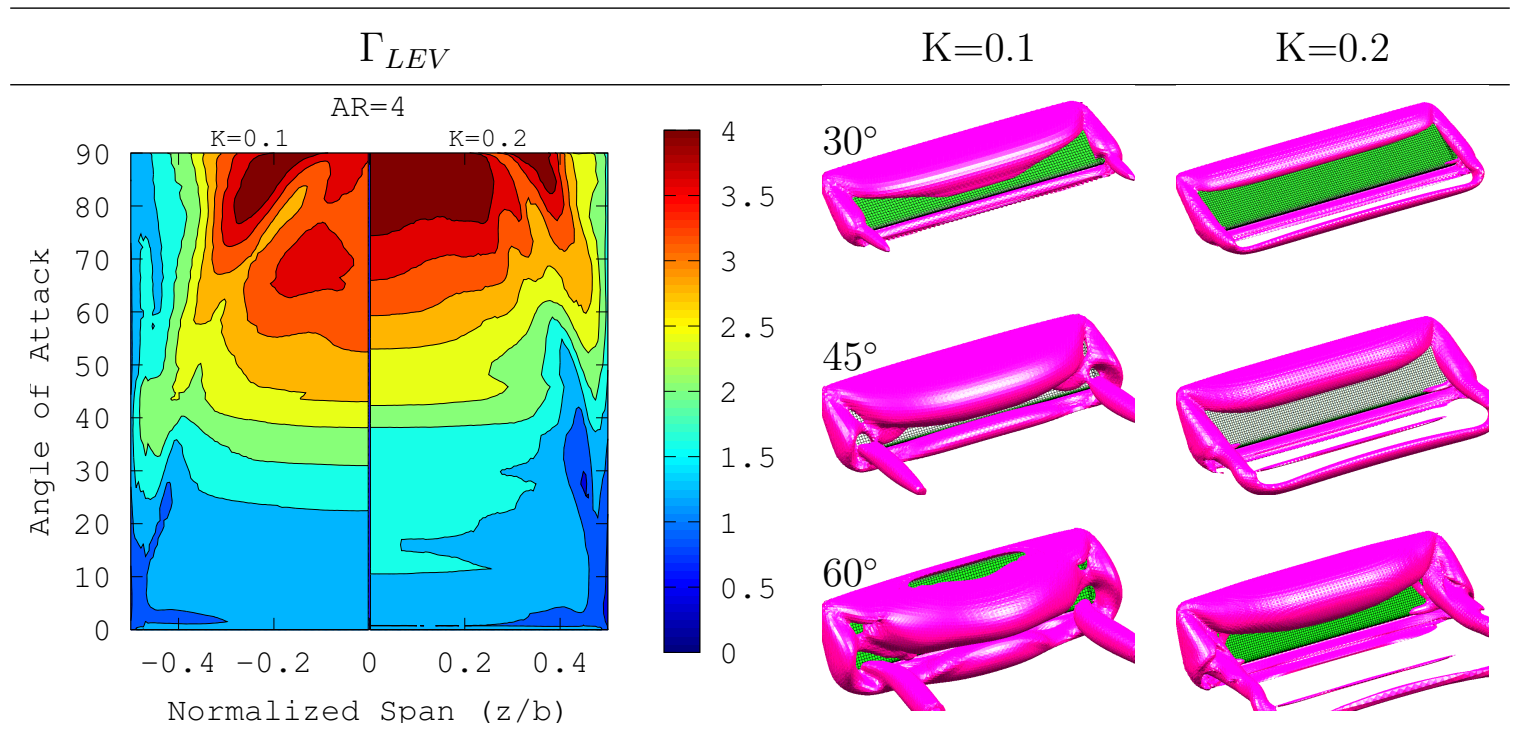

Figure 61: (Left) Contour of spanwise $\Gamma_{L E V}$ through entire pitching motion for the $A R=4$ wing at both tested $K$ values. (Right) Iso-surface contours where the $Q=5$ for both $K$ values at $30^{\circ}, 45^{\circ}$, and $60^{\circ}$

This section helps to establish the link between the aspect ratio, LEV circulation, and lift and drag slops. The lower lift slope on the $A R=1$ wing is mainly due to the weakened LEV by the tip vortices. As seen in figure 34 and 59, the LEV showed weaker mid-span vorticity, and lower span circulation. Through the pitching motion, the circulation never developed to levels seen at higher aspect ratios. As the aspect ratio increased, the LEV structure developed with less impedance from tip vortices and achieved higher circulation values across the span. This in turn allowed for the $\omega_{z}$ circulation to develop, resulting in a higher lift slope.

\section{Perching}

Previously with the pitching circulation plots, there were few discernible features shared between each aspect ratio. This was due to the effects of the tip vortices 
interacting with the LEV creating varying flow fields above each wing. With the introduction of the flow field deceleration (perching), each aspect ratio plot now shares similarities with one another. Primarily, as aspect ratio increases, the observed features increase in magnitude, and move proportionally to the span of the wing.

The $A R=1$ is comparatively different from its pitching counterpart. For the pitching case, $\Gamma_{C W}$ reached its peak at the end of the pitching motion. Due to the flow deceleration, $\Gamma_{C W}$ now peaks early in the perching motion, approximately $40^{\circ}$ as also seen in figure 58 for both pitch rates. The lower $K$ shows an additional circulation peak near the edge of the span between $30-50^{\circ}$. This secondary peak will also evident in the higher aspect ratios.

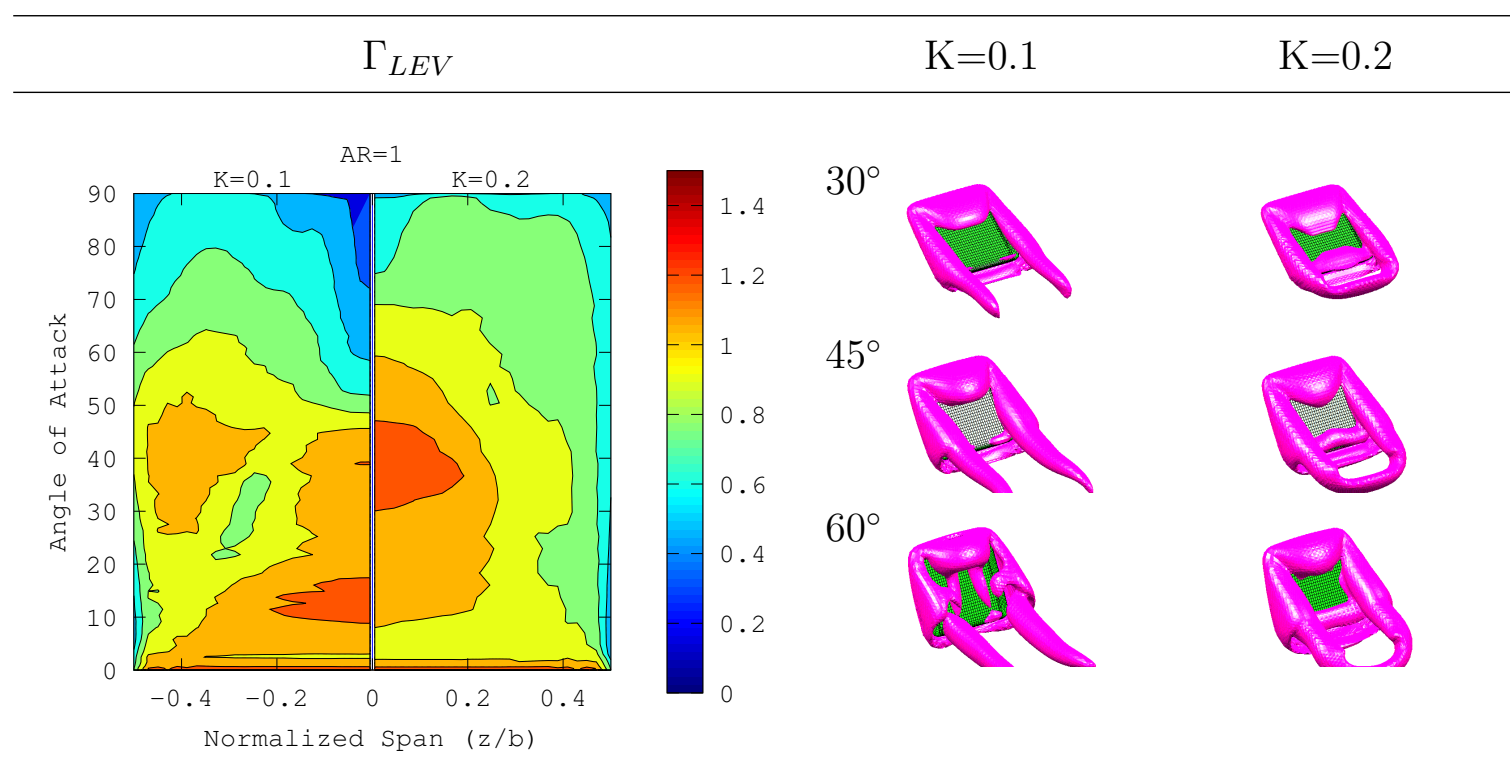

Figure 62: (Left) Contour of spanwise $\Gamma_{L E V}$ through entire perching motion for the $A R=1$ wing at both tested $K$ values. (Right) Iso-surface contours where the $Q=1$ for both $K$ values at $30^{\circ}, 45^{\circ}$, and $60^{\circ}$

The $A R=2$ primarily has increased contour magnitudes. The secondary peak in the $K=0.1$ has reappeared towards the edge of the span, while the center span 
magnitude has increased. The $K=0.2$ case also shows the increased span contours, with now the development of increased edge circulation. This edge circulation was not present in the $A R=1$ wing.

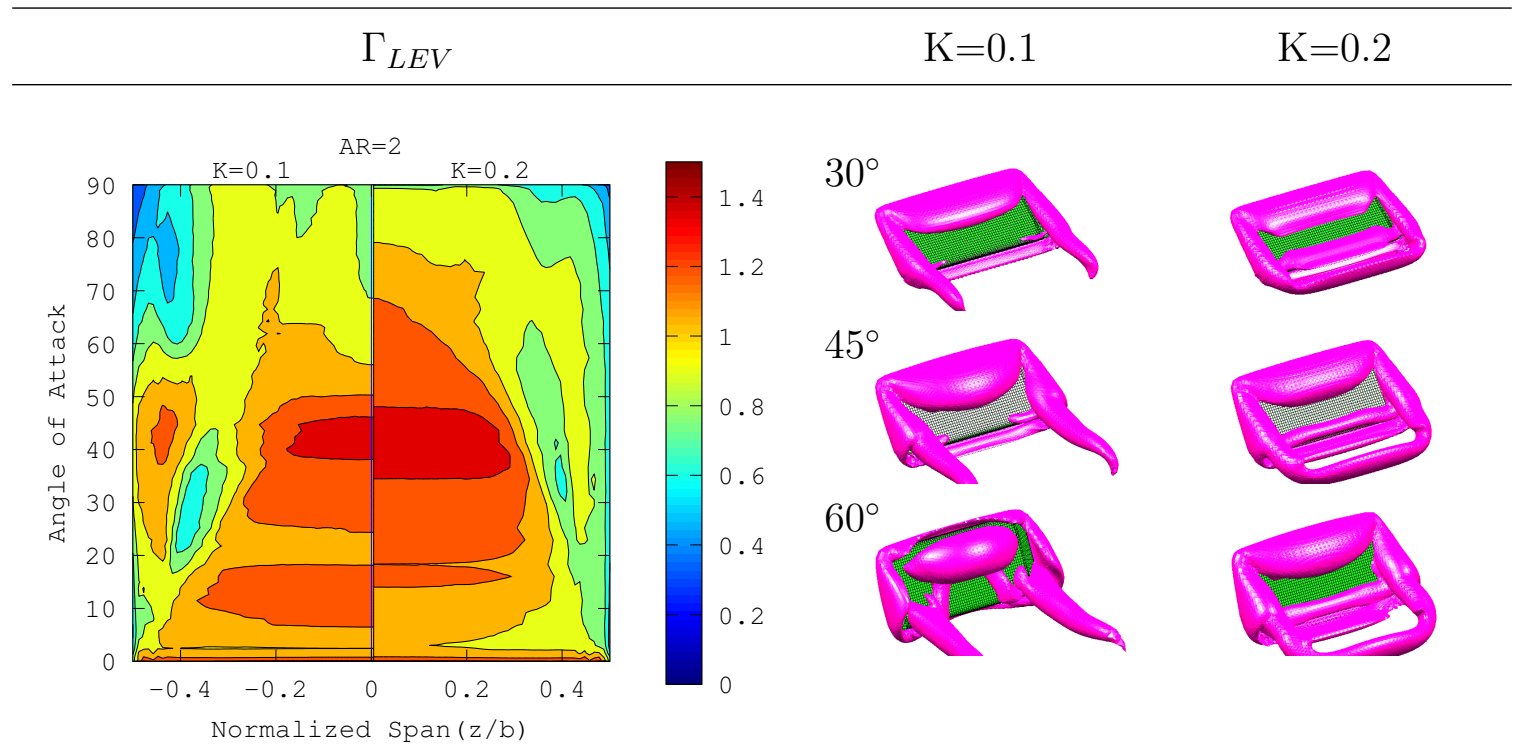

Figure 63: (Left) Contour of spanwise $\Gamma_{L E V}$ through entire perching motion for the $A R=2$ wing at both tested $K$ values. (Right) Iso-surface contours where the $Q=1$ for both $K$ values at $30^{\circ}, 45^{\circ}$, and $60^{\circ}$

Finally the $A R=4$ wing again shows increased circulation magnitudes, with the geometrically proportioned features that were evident with the $A R=2$ wing. A new noticeable difference between the two pitch rates is the extended duration of the $K=0.1$ mid-span circulation when compared to the 0.2 . This increased mid-span circulation does not mean the 0.1 rate produced higher lift. Due to the slower pitch rate, the LEV had an increased $\Gamma_{C W}$, but propagated away further from the plate at the end of the motion. This can be seen in figure 36. This increased distance decreases the effectiveness of the vortex's ability to induce lift on the wing. The $K=0.2$ was seen to be smaller, and closer to the plate, resulting in a weaker vortex 
that maintained its ability to induce lift.

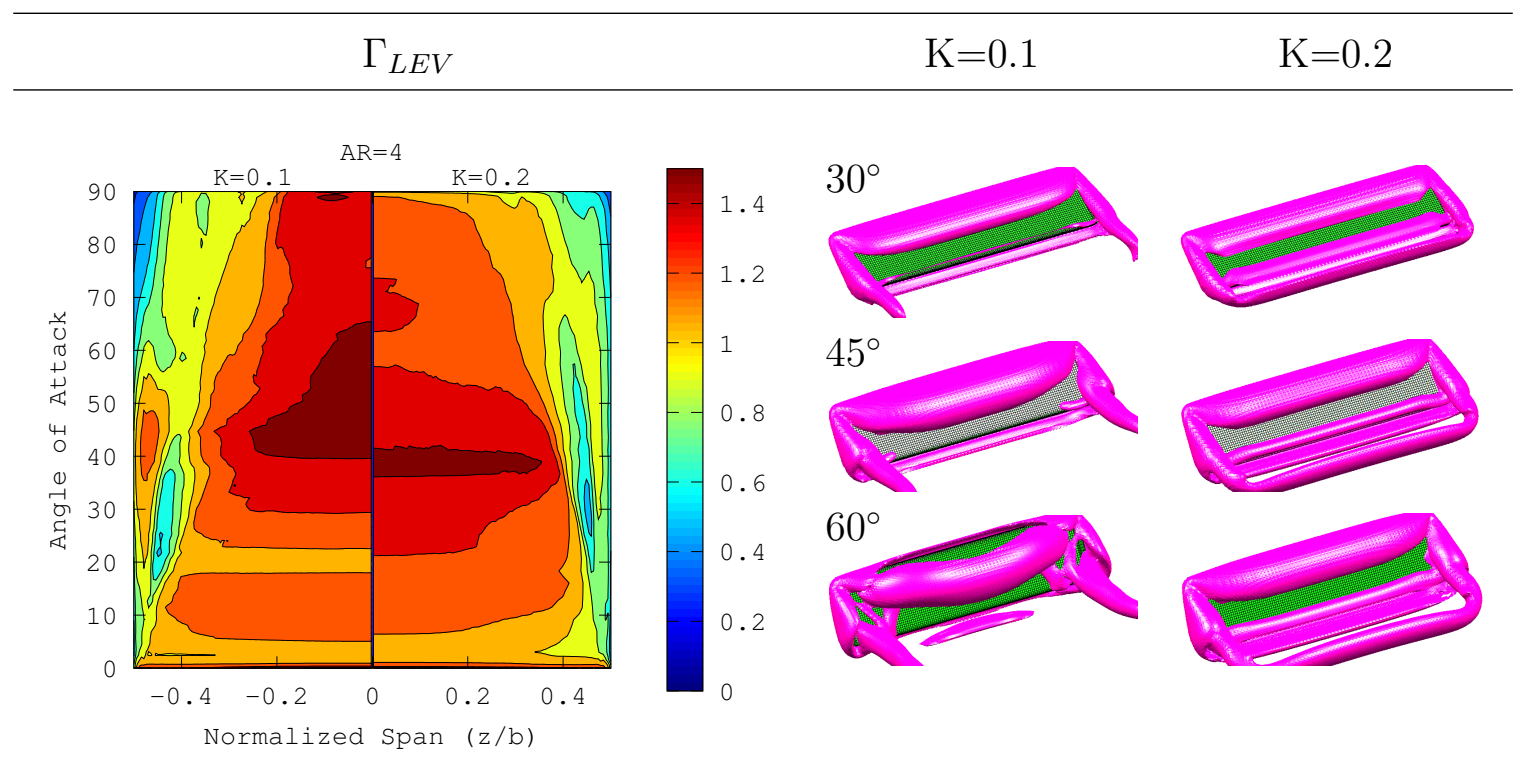

Figure 64: (Left) Contour of spanwise $\Gamma_{L E V}$ through entire perching motion for the $A R=4$ wing at both tested $K$ values. (Right) Iso-surface contours where the $Q=1$ for both $K$ values at $30^{\circ}, 45^{\circ}$, and $60^{\circ}$

\section{Circulation Conclusion}

In this chapter, the effect of the wing aspect ratio, reduced pitch rate, and pitching kinematics on the LEV circulation were examined.

Overall, the pitch-up spanwise circulation was shown to be dependent on the wing aspect ratio. The lowest aspect ratio wing $(A R=1)$ showed little to no coherent circulation in the contour, while the iso-surface plots showed a small LEV pushed off the surface by the tip vortices. For the $A R=2$ wing, spanwise circulation developed, but was not uniform. The effect of the tip vortices was evident by the inward pushing of the circulation. Finally, the circulation on the $A R=4$ was affected little by tip vortices. For the $A R=2$ case, the higher $K$ value caused a delay in circulation development. Tip vortex interaction was also shown affect less of the plate surface 
with an increase in $K$. This was most evident on the $A R=2$ and 4 wings, circulation would extend further across the span when compared to the lower $K$.

$K$ played an important role in the distribution and timing of development of spanwise circulation for the pitching motion. With an increase in $K$, spanwise circulation was extended further along the span, whereas the circulation with the lower $K$ dropped to lower magnitudes due to the presence of developed tip vortices. When the aspect ratio was equal to or greater than 2, the higher $K$ value led to a delay in spanwise circulation development for the pitch-up motion. This delay in circulation development resulted in a visibly smaller and less diffuse LEV that remained close to the wing's surface at higher angles of attack. Once the circulation started to develop, it increased at the similar rates between the two tested $K$ values. This suggested that the slope of circulation development depends on the aspect ratio, while the $K$ determines the timing of the circulation development.

Spanwise circulation for the perching plates followed a similar trend as the pitch up. As aspect ratio increased, circulation distribution become more uniform, with decreased influence from tip vortices. Evidence of vortex retention became evident on the lower $K$ cases by the prolongation of mid-span circulation acting for large intervals of perching motion. This retention of the vortex may play an acting part in the lift and drag production of the perching wing.

In summary, it was shown that the development of the LEV circulation for a wing undergoing a pitch-up motion depends on the aspect ratio of the wing. It was also shown that $K$ determines the timing of the development of the LEV: increasing $K$ results in a smaller LEV at higher angles of attack which remains close to the surface of the wing and contributes to higher lift. 


\section{CHAPTER IX}

\section{FEATURE-BASED ADAPTATION}

\section{A. Summary}

In this chapter, the dynamic stall phenomenon of a pitching NACA0015 airfoil is studied using a feature based grid adaption technique. The fluid solver is based on FUN3D which solves the three-dimensional, compressible, unsteady ReynoldsAveraged Naiver-Stokes equations. The one equation Spalart-Allmaras is used a the turbulence closure. The governing equations are discretized spatially using secondorder finite volume methods and temporally using an optimized second order backward difference scheme. The grid adaption is based the anisotropic tetrahedral adaptation approach in which grid is adapted to match a desired quality via an anisotropic metric calculated throughout the simulation. Particularly vorticity is tracked vorticity throughout the pitching cycle and adapt the grid in areas where vorticity is damped. Comparisons are also made with results using uniformly refined grids. Results suggest feature-based adaptation has potential in refining the mesh in the wake of the airfoil, allowing vorticity to be carried out several chords behind the airfoil without excessive dissipation. Our study also shows that great care must be placed in allowing the grid to be adapted in the vicinity of the airfoil as grid resolution can be lost.

\section{B. Importance of Wake Grid Resolution}

Research has shown that grid quality can dramatically affect simulation results. Kravchenko[82] showed that incorrect flow features were produced in the wake of a cylinder due to large grid spacing. As a result, the separated flow region was shorter 
and the recirculation region immediately behind the cylinder became smaller, resulting in incorrect flow features and poor force prediction. Similarly, in a review paper by Komerath [83], it was noted that the wake grid resolution behind helicopter blades can impact the accuracy of the flow simulation. In the study of dynamic stall, the grid quality also plays a critical role. Dynamic stall is a complex fluid mechanics problem plagued with vortex formation and shedding. To accurately predict the force coefficients, it is critical to capture the vortex formation and its propagation in the flow. However, without a priori knowledge of the vortex structures and their trajectories, creating a computationally efficient grid to capture these features is challenging.

Feature-based grid adaption is a promising technique to automatically adapt grid at regions of interests. It can reduce the manual interaction with the CFD tools and the required expertise of the user to obtain accurate solutions[84]. It can also significantly alleviate the need for spatial convergence verification of computational results by balancing the modeling accuracy and computational efficiency for engineering analysis. Kang et al. [85] and Park et al. [86] have successively implemented feature-based grid adaptation schemes to resolve vortex flow structures produced by a rotor-craft in hovering flight. Their studies showed that feature-based adaptation methods are able to improve the prediction of lift and drag coefficients by refining grid where vortices propagated through and interacted with vehicle structures. By improving spatial accuracy of the vortex structures, they showed that feature-based grid adaption can better model vortex interactions which are critical in hovering flight.

To fulfill the potential of feature-based grid adaption method, it is critical to calibrate the initial grid in order to simulate flow features correctly [87, 88]. Without proper evaluation of the initial grid, the adapt mesh can capture incorrect features far from their true locations. Further adaption can "force" features into a refined area and hence steadily enhance an erroneous solution with each grid adaption. It was hypothesized that this erroneous behavior was the result of an insufficient grid resolution of the initial uniform mesh on which the feature-based grid adaption was 
based. $[87,84]$

If properly addressed, such issues should not inhibit the application of mesh adaptation to problems in flow fields. [89] In order to confirm the effectiveness and viability of feature-based adaptation for dynamic stall flows, a study will be conducted on the flow over a 2D pitching NACA0015 airfoil. In particular, the study will compare the results of an uniformly refined grid to that of a feature adapted grid. To learn from previous studies, an initial spatial convergence study will be conducted to evaluate the effectiveness of uniformly refining a grid. This will be determined by investigating the change in coefficients of lift and drag. Next, one uniformly refined grid will be used as the initial grid for the adaptation study. The primary goal is to investigate if the mesh can be improved to better capture the wake structure of the pitching airfoil.

\section{Numerical Methods}

FUN3D $^{1}$ is an unstructured finite-volume solver that has been developed and supported by the NASA Langley Research Center. [1, 90]. FUN3D solves the threedimensional, unsteady, compressible Reynolds-Averaged Naiver-Stokes (RANS) equations in integral form:

$$
\frac{\partial}{\partial t} \int_{V} q d V+\int_{\partial V}\left(F^{*}-F_{V}\right) \cdot \hat{n} d S=0
$$

where $V$ is the control volume which is bounded by $\delta V . F^{*}$ and $F_{v}$ are the inviscid and viscous fluxes of conservative variables. $q$ represents the conserved variable vector

$$
q=\left[\begin{array}{c}
\rho \\
\rho u \\
\rho v \\
\rho w \\
E
\end{array}\right]
$$

\footnotetext{
${ }^{1}$ http://fun3d.larc.nasa.gov/
} 
For the case of a moving control volume, the convective fluxes must take in account the relative velocity of the control volume. The inviscid flux is adjusted for the control volume face speed $|W|$ such that:

$$
F^{*}=F-q W
$$

where $F$ is a stationary control volume flex vector. The volume average $(Q)$ of $q$ must be also taken to account for the moving volume:

$$
Q=\frac{\int_{V} q d V}{V}
$$

and the conservation equations become:

$$
\frac{\partial(Q V)}{\partial t}+\oint_{\partial V}\left(F^{*}-F_{v}\right) \cdot \hat{n} d S=0
$$

The convective inviscid and viscous fluxes are then defined as follows where $F^{*}$ now accounts for the relative motion of the control volume.

$$
\left.F^{*}=\left[\begin{array}{c}
\rho\left(u-W_{x}\right) \\
\rho u\left(u-W_{x}\right)+p \\
\rho v\left(u-W_{x}\right) \\
\rho w\left(u-W_{x}\right) \\
(E+p)\left(u-W_{x}\right)+W_{x} p
\end{array}\right] \hat{i}+\left[\begin{array}{c}
\rho\left(v-W_{y}\right) \\
\rho u\left(v-W_{y}\right)+p \\
\rho v\left(v-W_{y}\right) \\
\rho w\left(v-W_{y}\right) \\
+\left[\begin{array}{c}
(E+p)\left(v-W_{y}\right)+W_{y} p
\end{array}\right] \hat{j} \\
\rho\left(w-W_{z}\right) \\
\rho u\left(w-W_{z}\right)+p \\
\rho v\left(w-W_{z}\right) \\
\rho w\left(w-W_{z}\right) \\
(E+p)\left(w-W_{z}\right)+W_{y} p
\end{array}\right]\right\} \hat{k}
$$




$$
\begin{aligned}
F_{v}=\left[\begin{array}{c}
0 \\
\tau_{x x} \\
\tau_{y x} \\
\tau_{z x} \\
u \tau_{x x}+v \tau_{x y}+w \tau_{x z}-q_{x}
\end{array}\right] \hat{i}+\left[\begin{array}{c}
0 \\
\tau_{x y} \\
\tau_{y y} \\
\tau_{z y} \\
u \tau_{y x}+w \tau_{y z}-q_{y}
\end{array}\right] \hat{j} \\
+\left[\begin{array}{c}
0 \\
\tau_{x z} \\
\tau_{y z} \\
\tau_{z z} \\
u \tau_{z y}+w \tau_{z z}-q_{z}
\end{array}\right]
\end{aligned}
$$

Within FUN3D, the RANS equations are discretized using flux splitting scheme where the inviscid and viscous fluxes are handled separately. For second order accuracy, the values at the cell interfaces are calculated using gradients at the mesh nodes and computed using a least-squares technique. Currently, feature-based grid adaptation is only implemented on fully tetrahedral based computational domains. For tetrahedral meshes, the full viscous fluxes are discretized using a finite-volume formulation in which the required velocity gradients at the cell faces are computing using the Green-Guass theorem, which is equivalent to a Galerkin approximation.

\section{Temporal Discretization}

Time-accuracy is achieved through an optimized second order backward difference scheme (BDF2OPT). It is used to construct a higher order temporal scheme by extending the difference stencil in time[91]. The details of BDF2OPT and the temporal error control schemes are well documented by Biedron[1] and will be briefly discussed here. To begin the derivation of the equations used for time stepping, it is assumed that the control volume is invariant in time (the grid is rigid) which is written as 


$$
V \frac{\partial Q}{\partial t}=R
$$

Expanding on Eqn. (9.57) to evaluate the next time level of $n+1$, and writing the time derived as a series of backward difference levels gives

$$
\frac{V}{\Delta t}\left(\phi_{n+1} Q^{n+1}+\phi_{n} Q^{n}+\phi_{n-1} Q^{n-1}+\phi_{n-2} Q^{n-2}+\ldots\right)=R^{n+1}
$$

The series of $\phi_{n}$ governs the accuracy of the backwards difference of the temporal discretization. The respective values of $\phi_{n}$ varies depending the order of accuracy of the scheme, but must satisfy the requirement of $\sum \phi_{n}=0$. As stated before, FUN3D uses an optimized backwards difference scheme, which is a linear combination of second and third order coefficients. With an order of accuracy that is in between second and third order, the temporal equations are stable with any time-step. The coefficients for BDF2OPT are listed in Table 4.

\begin{tabular}{c|cccc} 
order & $\phi_{\mathbf{n}+\mathbf{1}}$ & $\phi_{\mathbf{n}}$ & $\phi_{\mathbf{n}-\mathbf{1}}$ & $\phi_{\mathbf{n}-\mathbf{2}}$ \\
\hline \hline $\mathbf{2}^{\text {nd }}$ & $3 / 2$ & -2 & $1 / 2$ & 0 \\
$\mathbf{3}^{\text {rd }}$ & $11 / 16$ & -3 & $3 / 2$ & $-1 / 3$ \\
$\mathbf{B D F}_{\mathbf{o p t}}$ & $3 / 2-\phi_{n-2}$ & $-2+3 \phi_{n-2}$ & $1 / 2-3 \phi_{n-2}$ & $-0.58 / 3$
\end{tabular}

TABLE 4

Coefficients for backwards difference schemes [1]

A pseudo-time term $(\tau)$ is introduced into Eqn. (9.58), as in Ref. [92], to minimize the error due to the linearization about the time level of $n+1$. If $\delta Q$ is linearized about $\tau$ and assumed to be equal to $\delta Q^{m}=Q^{m+1}-Q^{m}$, where $m$ is the current pseudo-time level, then the final form of the temporal equation can be written as 


$$
\begin{aligned}
& {\left[\left(\frac{V}{\Delta \tau}+\frac{V \phi_{n+1}}{\Delta t}\right) I-\frac{\partial R^{m}}{\partial Q}\right] \Delta Q^{m}=R^{m}-} \\
& \quad \frac{V}{\Delta t}\left(\phi_{n+1}\left(Q^{m}-Q^{n}\right)+\phi_{n-1}\left(Q^{n-1}-Q^{n}\right)+\phi_{n-2}\left(Q^{n-2}-Q^{n}\right)+\ldots\right)
\end{aligned}
$$

At each sub-iteration of $m$, the linear system in Eqn. (9.59)is iteratively solved using a user-specified number of point Jacobi or Gauss-Seidel sweeps. When between time-steps, the equations are advanced in pseudo time with local time stepping to accelerate the solution to a steady state pseudo time. In order to accelerate convergence in pseudo time, the CFL number can be ramped during the sub-iterations.

A temporal error control method[1] has been used to reduce the number of subiterative loops by exiting the dual time stepping process when a specified criteria has been met. This helps address the issue of the choice of the number of sub-iterations to use when solving the flow equations. The temporal error is calculated by examining the difference in residual contribution of two different levels of time derivative approximations. The sub-iteration loop will be terminated when the residuals drop below a specific fraction of the temporal error norm.

\section{Feature-Based Adaptation}

FUN3D's feature-based adaptation is a node based approach that uses anisotropic tetrahedral adaptation. The mesh resolution is modified to match a desired quality via a anisotropic metric calculated throughout the simulation.[84, 93, 94] The approach in this paper focuses on using the vorticity magnitude at each node to determine the local and global adaptation metric. First, the vorticity magnitude is used to form an adaptation key, $K_{l, \omega}$. This key is the vorticity magnitude delta across an edge connecting two adjacent nodes $n_{1}$ and $n_{2}$, which is then scaled by the edge length, $l_{e}$.

Scaling $K_{l, \omega}$ by the edge length acts as a filter, emphasizing large changes in vorticity across long edges, while reducing the change across smaller edges. This assists in the adaptation of large cells first. 


$$
K_{l, \omega}=l_{e} \frac{\left|\omega_{n 1}\right|-\left|\omega_{n 2}\right|}{2}
$$

The local error adaptation intensity, $\hat{\mathrm{I}}$, is then computed a for each node in the domain. This is done by finding the maximum scaled key at each node where the adaptation key is normalized by a user specified tolerance, $K_{t}$, thus:

$$
\hat{I}=\max \left(\frac{K_{l, \omega}}{K_{t}}\right)_{e d g e}
$$

The selected $\hat{\mathrm{I}}$ is the edge with the greatest scaled vorticity delta connected to the node Next, the new isotropic mesh size, $h_{1}$, is calculated using an estimate of the spacing from the original mesh, $h_{0}$, and a coarsening factor $C$. In this study $C=1.1$.

$$
h_{1}=h_{0} \min \left(C,\left(\frac{1}{\hat{I}}\right)^{0.2}\right)
$$

Finally, the anisotropic adaptation metric is derived using the scalar isotropic mesh size (Eqn. (9.62)) and a vorticity-magnitude Hessian not discussed here. The derivation of the anisotropic metric is detailed thoroughly in references [95, 96]. During the computation of the pitching motion, the metric is intersected in time to track the areas that require adaptation. This allows areas vorticity propagates through to be marked for adaptation. The metric is then passed to adaptation modules currently implemented in FUN3D to modify the mesh. The adaptation process provides node insertion and deletion, edge swapping, and node movement to achieve the the desired mesh density and quality necessary to match the derived metrics. The mesh adaptation process has been described in several papers.[84, 96, 97]

The adaptation modules implemented in FUN3D haven been shown to work well for both static inviscid[84] and viscous flows [98]; however, viscous adaptation lacks the capability to modify the the surface geometry. The mesh can only be modified outside of the cells that define the boundary layer across the surface geometry. Thus it is up to the user to ensure the surface geometry is adequately defined to 
capture the near wall boundary layer of the geometry. The practitioner can control how much of the boundary layer mesh is adapted by freezing the mesh a specified distance away from the surface. This capability has also been used in Ref.[98], and will be used in this study.

\section{Richardson Extrapolation}

Preforming sensitivity studies on the spatial schemes of a numerical simulation can sometimes be prohibitively computational expensive to conduct. Numerical data is normally compared to experimental data to validate the computed solution. However, experimental data is not always available, thus alternate routes must be pursed to ensure that the simulations are converging onto a single answer. One possible method is to use Richardson Extrapolation [99] to estimate the fully converged answer across two or three successively refined grids by a constant factor. The extrapolation technique is used to estimate the order of the solution, and project out a fully converged solution. This can help the practitioner estimate the error associated with the simulation and determine whether further grid refinement is required.

The method to estimate the converge solution is straight forward and can be done two different ways. The first, if the discretization order of solution is known, only two grids with some refinement ratio $r$ (for this study: 1.5 ) is required. The converged solution can be found with

$$
f_{h \approx 0} \cong f_{1}+\frac{\left(f_{1}-f_{2}\right)}{\left(r^{p}-1\right)}
$$

Where $f_{h \approx 0}$ is the exact solution for an infinitely refined grid or time step, $p$ is the formal order of accuracy of the spatial discretization, and $f_{1}$ and $f_{2}$ are the flow variables of interest on the fine and coarse grid respectively. In the event that $p$ is not known, it can be estimated with a three refined grids that are refined at a constant refinement ratio $(r)$ value. To estimate $p$ 


$$
p=\frac{\ln \left(\frac{f_{3}-f_{2}}{f_{2}-f_{1}}\right)}{\ln (r)}
$$

where $f_{1}$ to $f_{3}$ are the flow variables of interest on the finest to coarsest grid. Once $p$ is known, Eqn. 9.63 can be used. To estimate the error associated with the fine grid, Eqn. 9.63 can be rewritten as

$$
E_{\text {fine }}=\frac{\left(f_{1}-f_{2}\right)}{\left(r^{p}-1\right)}
$$

And the error for a coarse grid(s) can be found with:

$$
E_{\text {coarse }}=\frac{r^{p}\left(f_{1}-f_{2}\right)}{\left(r^{p}-1\right)}
$$

\section{E. Grid Generation}

The NACA0015 geometry was selected for this study as it was used in the referenced experimental work. [33] A family of grids were generated using AFLR3[100] for the spatial convergence study. AFLR3 (Advancing-Front/Local-Reconnection) is a unstructured tetrahedral element grid generation code. An initial grid was uniformly refined by a constant factor of 1.5. Thus each newly created grid had the number of nodes defining the airfoil surface, and outer boundary spacing was increased by a $r$ factor of 1.5: ie if the outer boundary had 32 nodes, the new boundary would have 48. Each grid was generated only with tetrahedral elements due to limitations in the feature-based adaptation in FUN3D. With these settings, the family of grids generated are presented in Table 5. 


\begin{tabular}{ccccccc} 
& \multicolumn{2}{c}{ Trailing } & Outer & Initial Cell & & \\
$\mathbf{h}$ & Airfoil & Edge & Boundary & Height $\left(10^{-5}\right)$ & $\boldsymbol{g}_{\boldsymbol{r}}$ & Nodes \\
$\mathbf{1}$ & 128 & 2 & 32 & 5.693 & 1.4000 & 5626 \\
$\mathbf{2}$ & 192 & 3 & 48 & 3.795 & 1.2515 & 16878 \\
$\mathbf{3}$ & 288 & 5 & 72 & 2.530 & 1.1613 & 36546 \\
$\mathbf{4}$ & 432 & 8 & 108 & 1.687 & 1.1048 & 82284 \\
$\mathbf{5}$ & 648 & 12 & 162 & 1.124 & 1.0687 & 185292 \\
$\mathbf{6}$ & 972 & 18 & 243 & 0.749 & 1.0453 & 419258
\end{tabular}

TABLE 5

Geometric settings for NACA0015 spatial study

Where $g_{r}$ is the geometric ratio of the boundary layer, and $h$ is the current grid number. An example of the family of grids created is shown in figure 65 . 

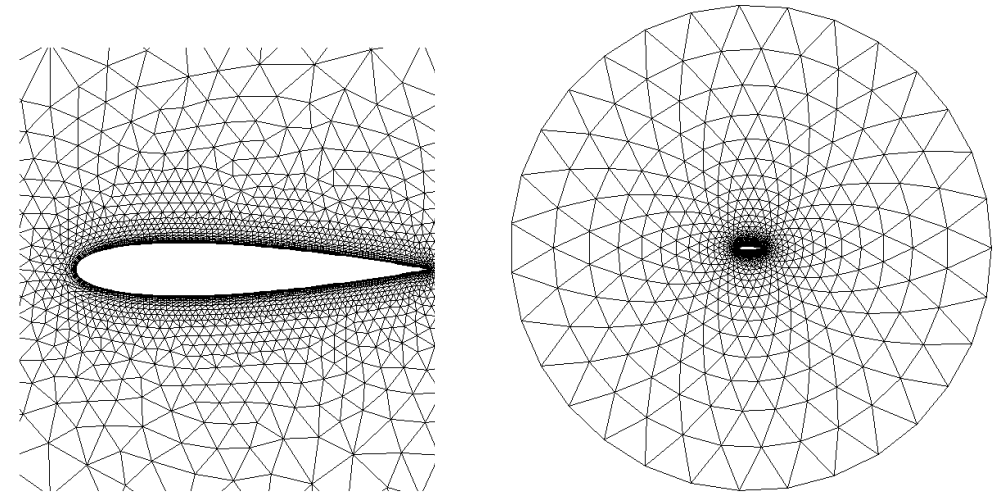

(a) Initial Grid $\left(h_{1}\right)$

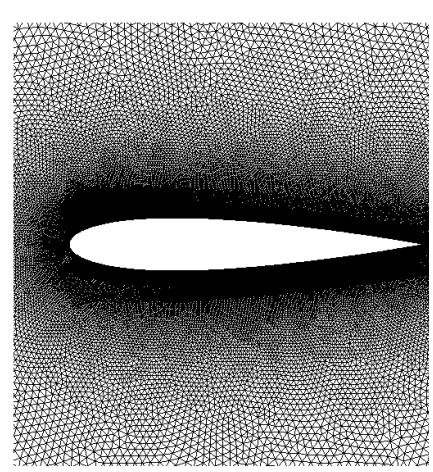

(c) Refined Grid $\left(h_{5}\right)$ (b) Initial Domain $\left(h_{1}\right)$

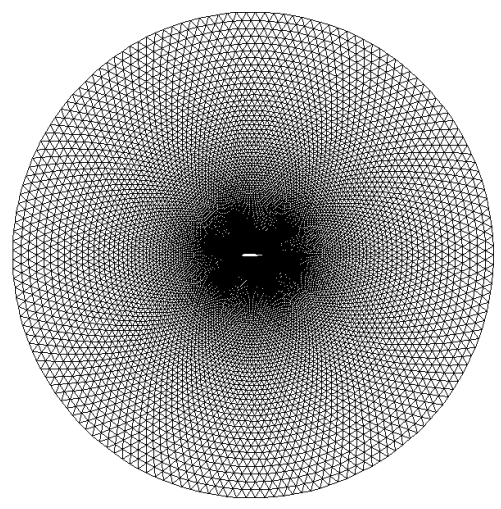

(d) Refined Domain $\left(h_{5}\right)$

Figure 65: Uniform Grid Refinement Around Airfoil

\section{Flow Conditions and Kinematics}

The flow conditions, as with the pitching kinematics are listed in Table 6 have been taken from Piziali[33]. The airfoil is pitched about the quarter-chord location following a the sinusoidal kinematic described as 


$$
\alpha=\alpha_{m}+\alpha_{0} \sin \left(\frac{2 \pi k M_{\infty} t}{c}\right)
$$

where $\alpha$ is the instantaneous angle of attack, $\alpha_{m}$ is the mean angle of attack, $\alpha_{0}$ is the pitch amplitude, $k$ is the reduced pitching frequency, $M_{\infty}$ is the free stream Mach number, $t$ is the simulation time, and $c$ is the airfoil chord length.

\begin{tabular}{ccccc}
$\alpha_{\mathbf{m}}$ & $\alpha_{\mathbf{0}}$ & $\mathbf{k}$ & $\mathbf{M}_{\infty}$ & $\mathbf{R e}\left(\mathbf{1 0}^{\mathbf{6}}\right)$ \\
\hline 13.07 & 4.27 & 0.134 & 0.289 & 1.947
\end{tabular}

TABLE 6

Flow conditions and kinematics used for pitching airfoil

For the temporal sensitivity analysis, the time step was also varied. If the time step is too large flow features may be improperly resolved, if too small, then computational resources could be wasted. Like the grid generation, the time step was consecutively refined, but by a factor of 2 . Five levels of refinement were chosen to test along side the spatial study, and are listed in Table 7. As $i$ increases, so does the number of steps taken per pitching cycle. The time step $\Delta t$ varied from 0.05 to 0.8 , which corresponds to the flow translating $1 \%$ to $23 \%$ of the airfoil's chord $(c)$.

\begin{tabular}{c|ccccc}
$\mathbf{i}$ & $\mathbf{1}$ & $\mathbf{2}$ & $\mathbf{3}$ & $\mathbf{4}$ & $\mathbf{5}$ \\
\hline steps & 100 & 200 & 400 & 800 & 1600 \\
$\boldsymbol{\Delta} \mathbf{t}$ & 0.8 & 0.4 & 0.2 & 0.1 & 0.05 \\
$\boldsymbol{\Delta x} / \mathbf{c}$ & 0.23 & 0.12 & 0.06 & 0.03 & 0.01
\end{tabular}

TABLE 7

Tested time steps $(\Delta t)$ used for temporal analysis, and the estimated flow convection distance per time step $(\Delta x / c)$. 


\section{F. Uniform Grid Refinement}

Before beginning the study on the pitching motion, the solver was benchmarked to ensure spatial convergence would be seen with a static airfoil. The flow conditions matched the conditions specified in Table 6 and the selected turbulence solver was the one equation Spalart Allmaras. Figure 66 reports the steady state force coefficients at $\alpha=13.04^{\circ}$ against $\left(N^{-1}\right)^{\frac{1}{2}}$, where $N$ is the number of nodes in the mesh, reported in Table 5. This assumes the characteristic edge length of the mesh, $h$, varies with the inverse of the square root, $h \approx N^{-1 / 2}$, for a $2 \mathrm{D}$ grid. The spatial discretization is also assumed to be second order, $p=2$, thus flow variables of interest should vary with $h^{2} \cdot[84]$

In figure 66, the bottom axis can be read from left to right moving from the finest grid to the coarsest $\left(h_{6} \leftarrow h_{1}\right)$. The two plots show as the grid is refined, lift and drag coefficients begin to converge. For comparison, the Richardson Extrapolation is shown for a $h=0$ grid. This simply verifies that as the grid is refined, the flow solution begins to converge onto a single answer.
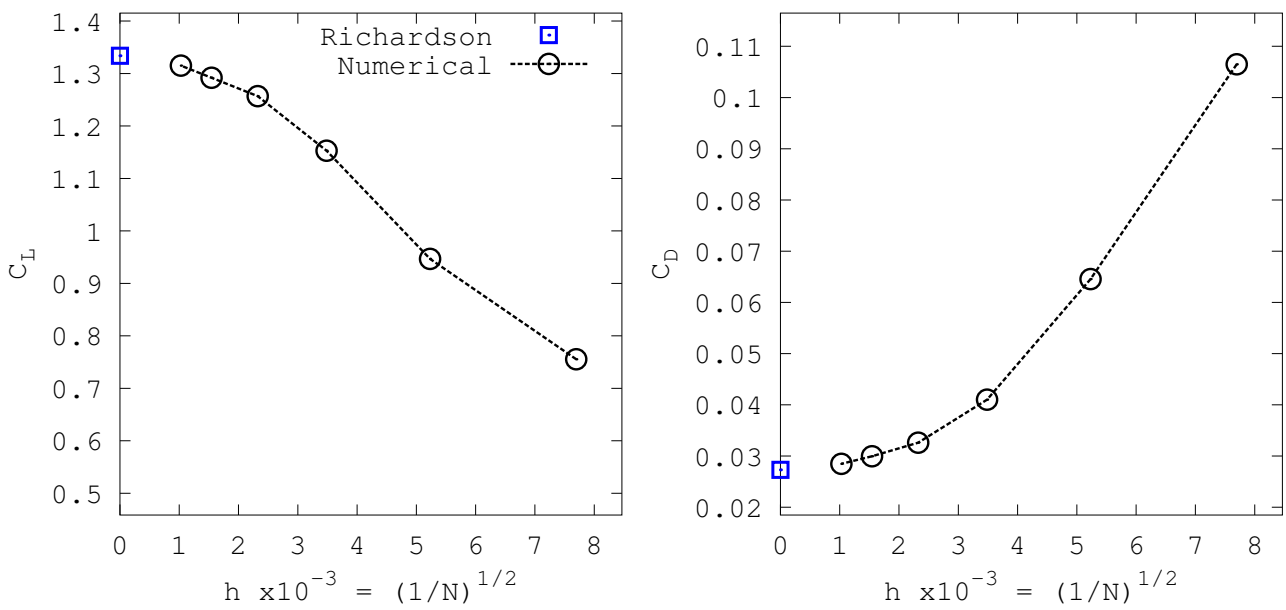

Figure 66: Static lift, drag, and moment coefficients at an angle of $13.04^{\circ}$.

Figure 67 illustrates how the flow changes with uniform refinement. It can be 
seen that a large separation region is prevalent about the airfoil in figure 67a and is greatly reduced in figure $67 \mathrm{~d}$. This helps illustrate the importance of grid resolution. The flow features can dramatic change with the grid used.

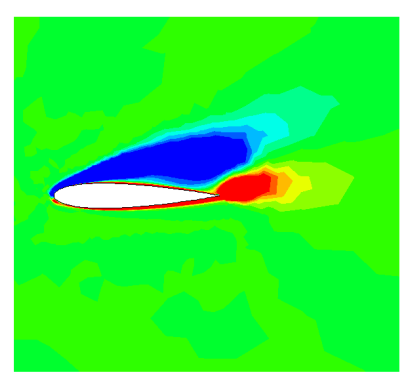

(a) $h_{1}$

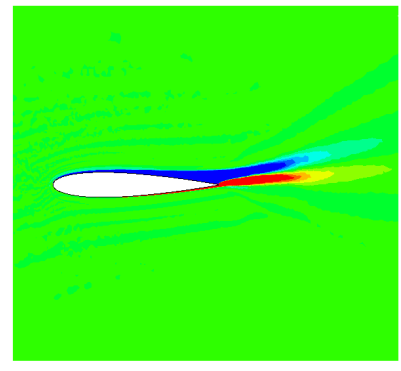

(c) $h_{5}$

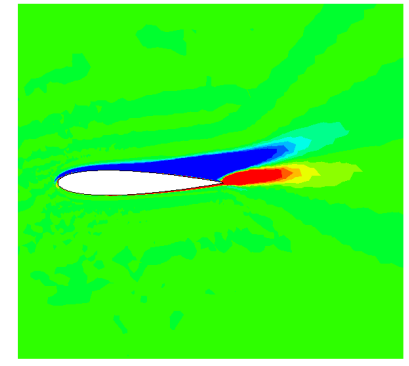

(b) $h_{3}$

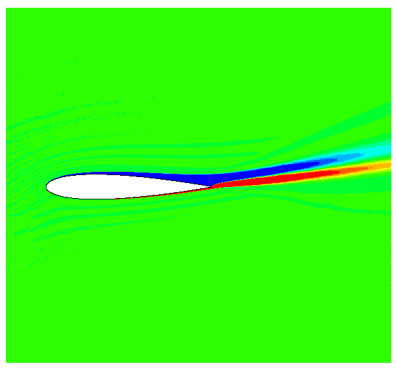

(d) $h_{6}$

Figure 67: Changes in vorticity with grid resolution of a steady state angle of attack at $13.04^{\circ}$

\section{1. $13 \pm 4^{o}$ Pitching Convergence}

In this section, the spatial and temporal convergence of a pitching airfoil will be focused on. Each grid specified in Table 5 was tested at each time-step listed in Table 7 , resulting in 36 unique combinations of grid density and time step sizing. It is important to note: results from grid combinations $h_{1}, h_{2}, \Delta t=0.4,0.8$ will not be discussed in the future sections.

Figures 68 and 69 show the averaged coefficient of lift and drag when the 
spatial and temporal resolutions are varied respectively. Figure 68 plots the effects of grid density on the averaged lift and drag coefficients. Lift, and drag show a strong dependence on the number of nodes used. As the spatial resolution of the grid is increased, the magnitude of the averaged forces begin to approach to the extrapolated value.

Temporal resolution, figure 69 , has little effect on the averaged force coefficients. At each grid resolution, reducing the time step modifies the averaged force coefficients little. Examining the instantaneous force coefficients,figure 70, throughout the pitching cycle reveals a very different story. It can be seen that as the spatial resolution is increased, the magnitude of the forces is modified but the temporal resolution has control over the shape of the force profile through the cycle. As the spatial resolution is increased, the flow features are better resolved due to increased grid density, but can be damped due to a coarse temporal resolution . Thus the flow field can be adequately resolved spatially, but not properly tracked through time. As time is refined, the flow feature is better projected altering the shape of the force profile through time.
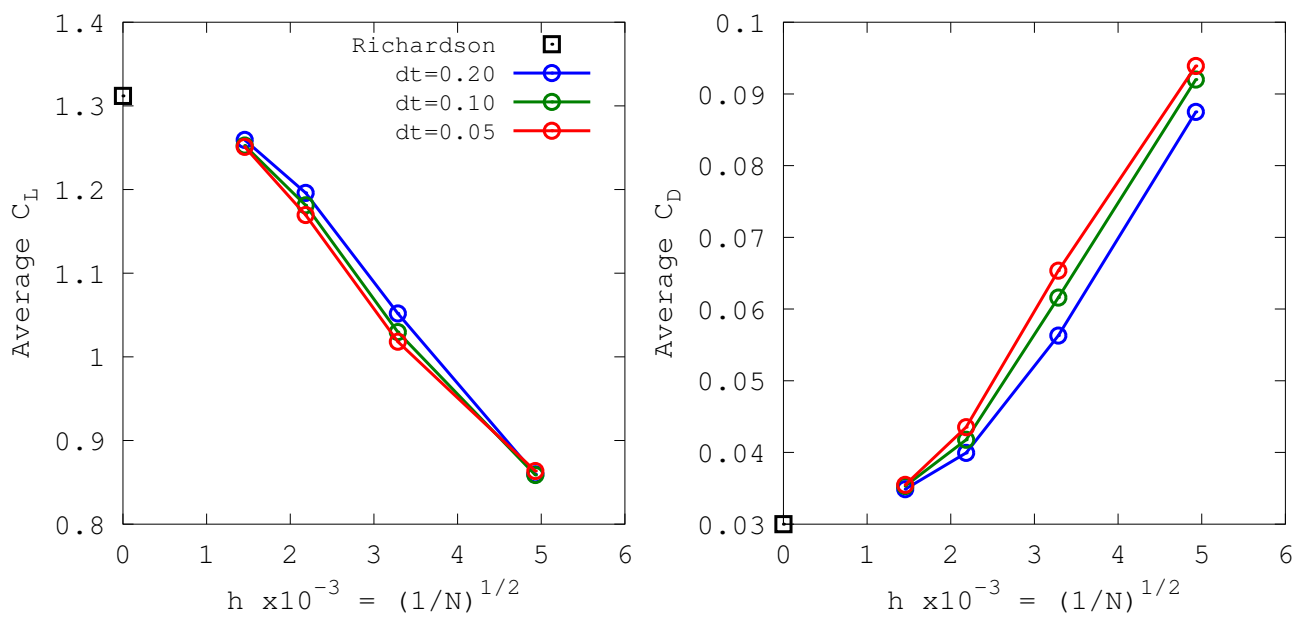

Figure 68: Lift, and drag coefficient variation due to selected spatial resolution of a pitching airfoil between $13 \pm 4^{\circ}$. 

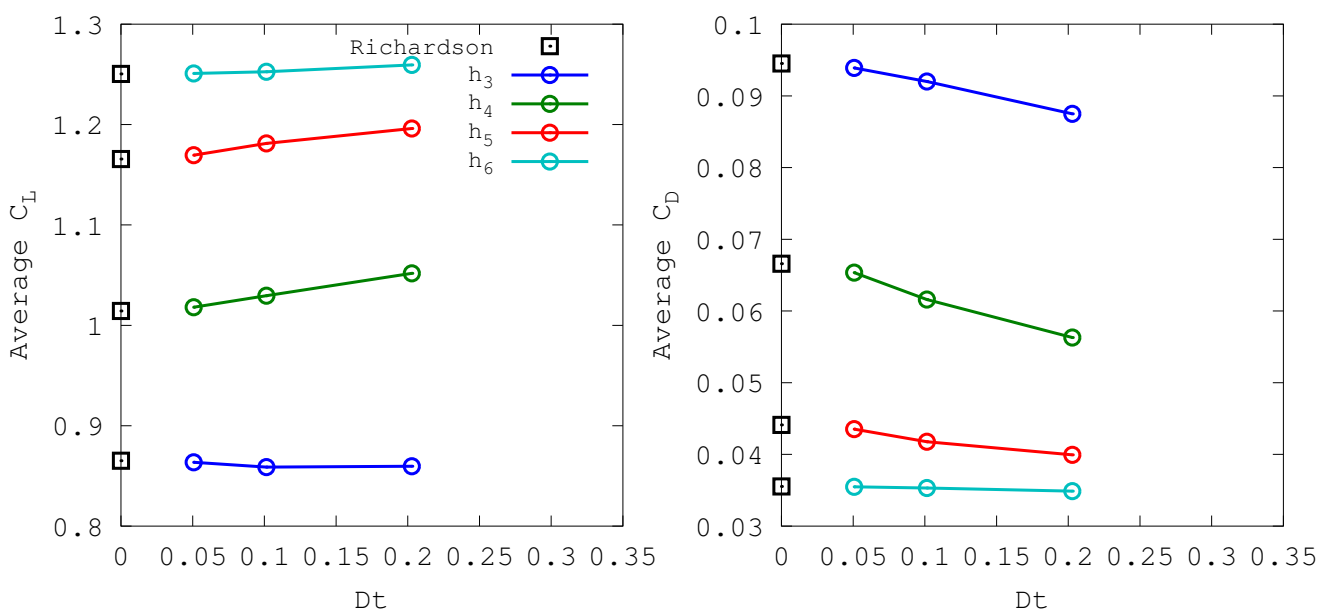

Figure 69: Lift, and drag coefficient variation due to selected temporal resolution of a pitching airfoil between $13 \pm 4^{\circ}$.

\section{Convergence Error}

In order to conclude which grid and time step combination would give a satisfactory result, the error associated with each spatial and temporal combination must be compared. The error for averaged lift and drag coefficients were calculated using Eqn. 9.65 and 9.66 are reported in figure 71. The spatial error of the lift and drag coefficients (figure 71a) is seen to plateau as the grid density approaches $h_{6}$. As discussed in the last section, the temporal resolution has a greater impact on the instantaneous force coefficients rather than the averaged. Examining the temporal error (figure 71b), the decrease in error becomes linear as the grid is refined and the time step is reduced. However, the magnitude of error associated the averaged coefficients due to the temporal resolution is nearly two magnitudes lower than the spatial related error. Thus the time step should be chosen from examining the instantaneous force history and not the averaged error. 

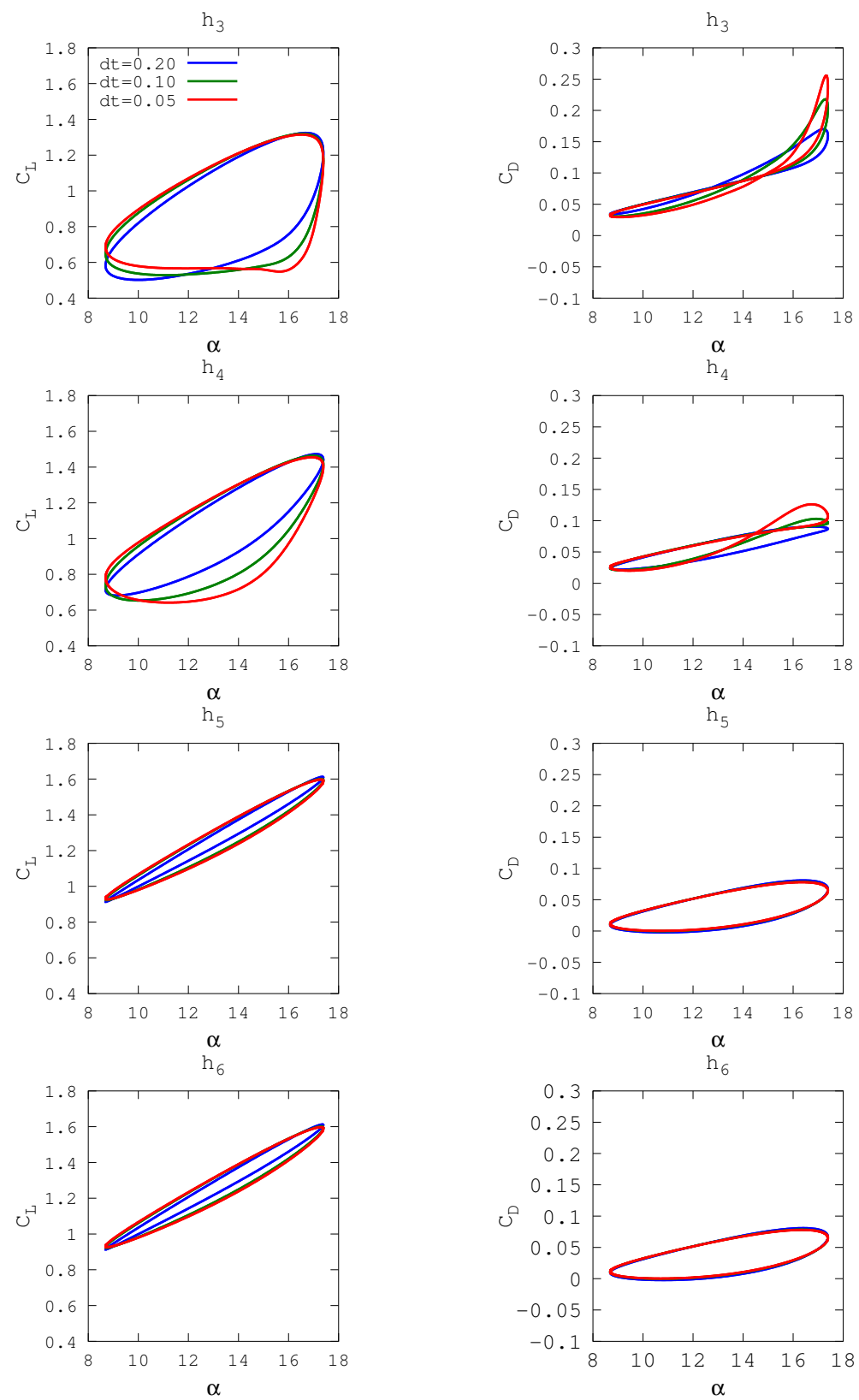

Figure 70: Lift, and drag coefficient of a pitching airfoil between $13 \pm 4^{\circ}$ at $\Delta t=$ $0.05,0.1$, and 0.2 . 

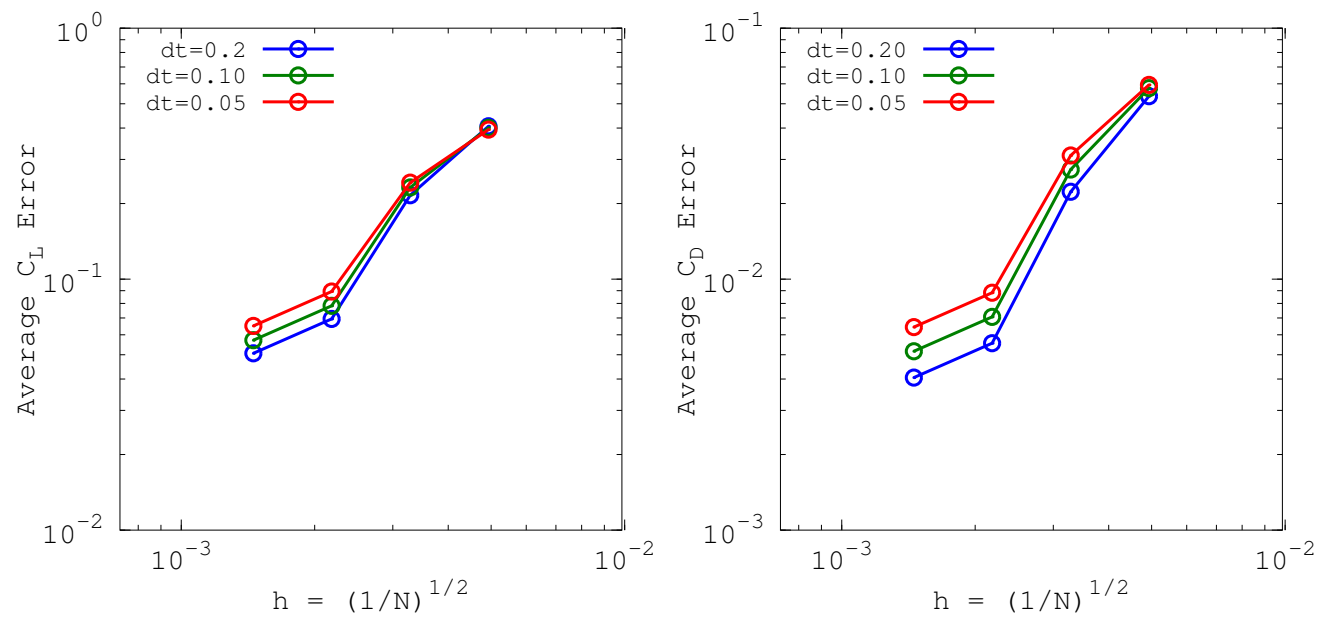

(a) Lift and drag coefficient error due to grid density.
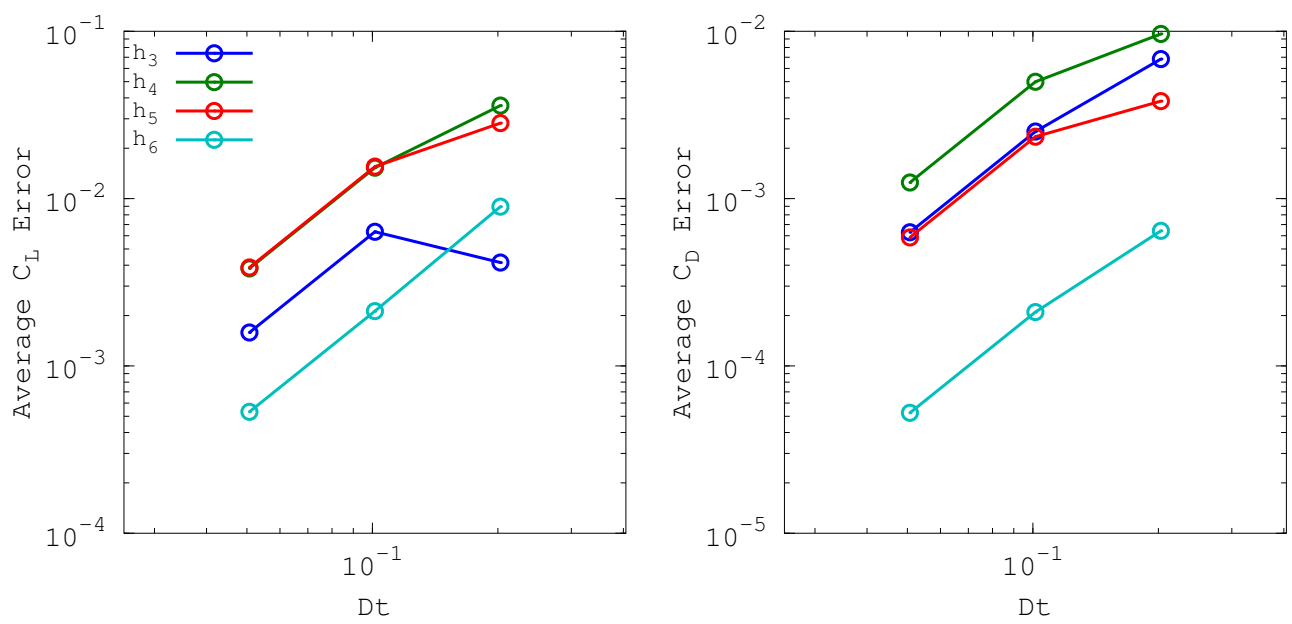

(b) Lift and drag coefficient error due to time step.

Figure 71: Computed lift and drag coefficient errors due to selected spatial and temporal resolutions.

\section{G. Feature-Based Adaptation}

In order to investigate whether feature adaption can accelerate the reduction of spatial error using less nodes, a grid outside of the asymptotic convergence of 
lift and drag was selected. To prevent erroneous solutions from being developed from an under-refined grid, $h_{4}$ was selected due to being outside of the lift and drag convergence seen in figure 70 and 71a. Selecting $h_{4}$ for refinement will demonstrate whether feature-based adaptation is able to bring a grid $\left(h_{4}\right)$ to a similar solution predicted by $\left(h_{6}\right)$ without input by the user. A time step of $\Delta t=0.1$ was used, and all flow conditions remained equal to those specified in Table 6 .

Before discussing the settings used, the process used to determine the adaptation metric must be introduced. Figure 72 illustrates the iterative process used. An initial grid, $h_{4}$, is supplied to the flow solver. After each time step, the adaptation metric is computed with the current flow solution, and then intersected with the previous calculated metric. The flow solution is advanced in time, and the grid moved. This process repeats until four pitching cycles were completed. The adaptation metric is then passed to the grid adaptation modules implemented in FUN3D. The grid is then iterated until the adaptation metric is satisfied. The grid is then saved, and passed back to the flow solver and the flow entire process repeats.

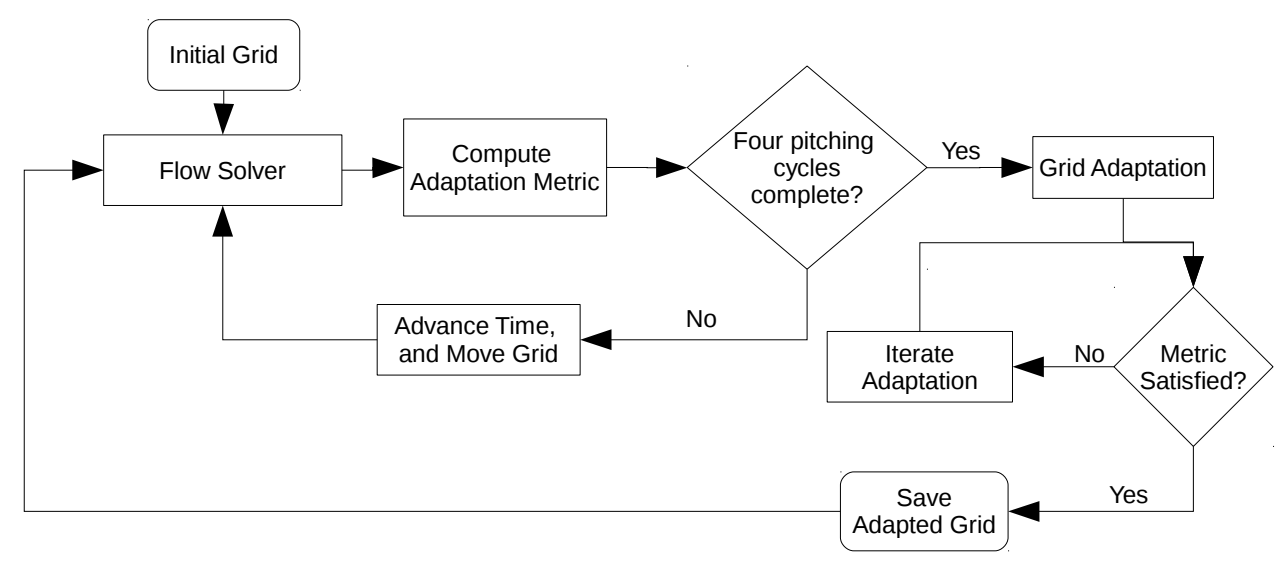

Figure 72: Feature-based adaptation process

Each flow solver and adaptation iteration possessed the same flow settings 
as previously stated, and was allowed to iterate 6 times. During each iteration, the adaptation was allowed to increase, or decrease cell volume by moving, adding and/or removing nodes, or swapping edges. The maximum desired node count was set to an equivalent node count of $h_{6}(\approx 400,000$ nodes $)$ after 6 iterations. Each iteration allowed the grid to increase the global node count in increments of $18 \%$. This did not mean the adapted grid reached this maximum node count. At each adaptation iteration, the grid was only allowed to grow at a specified rate to ensure needless grid growth was avoided. The boundary layer was frozen at a y+ distance of 100 to keep the boundary layer from being modified.

\section{Adapted Mesh}

Before discussing the results, the adapted mesh produced by the feature adaptation will be examined. Not all meshes will be shown, as it would be superfluous. Figure 73 shows three meshes from a selection of iteration steps 0 (initial), 4 , and 6 . Comparing the initial and the $4^{\text {th }}$ iteration of the mesh, it is easily seen there has been significantly modification. Adaptation has mainly occurred in the wake and around the airfoil, with little adaptation occurring elsewhere. Comparing steps 4, and 6 , it is difficult to distinguish any change in the mesh. It was confirmed through the examination of the global node count that the node density was increased, and can be seen in figure 74. To better observe how the grid was adapted, the change in cell volume was examined. 

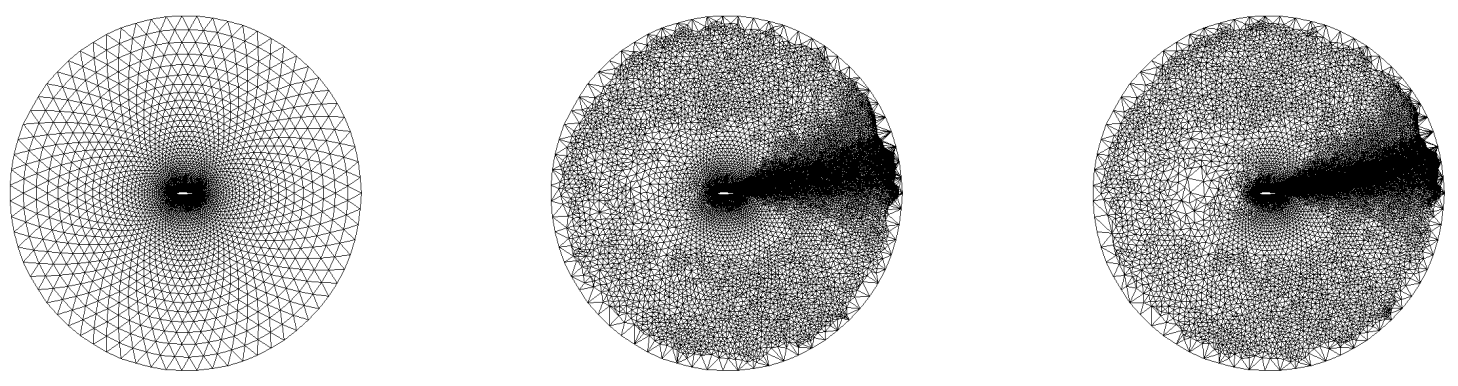

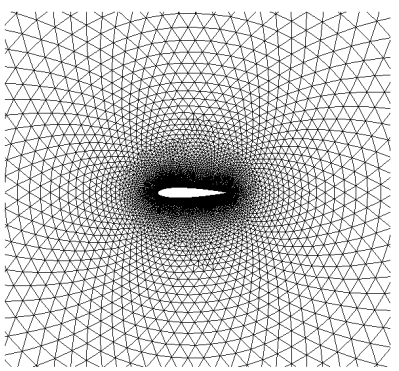

(a) Initial Grid

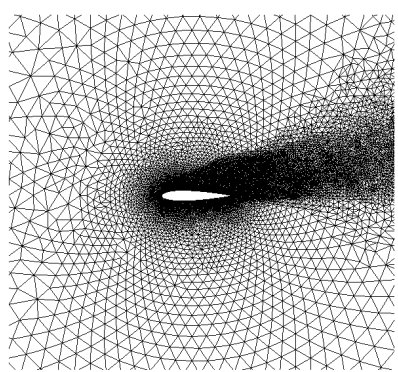

(b) $4^{\text {th }}$ Adaptation

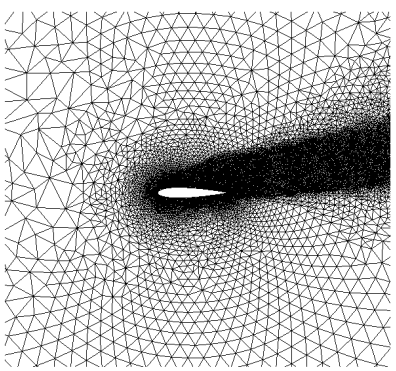

(c) $6^{\text {th }}$ Adaptation

Figure 73: The initial, $4^{\text {th }}$, and $6^{\text {th }}$ mesh adaptation of the selected $h_{4}$ grid.

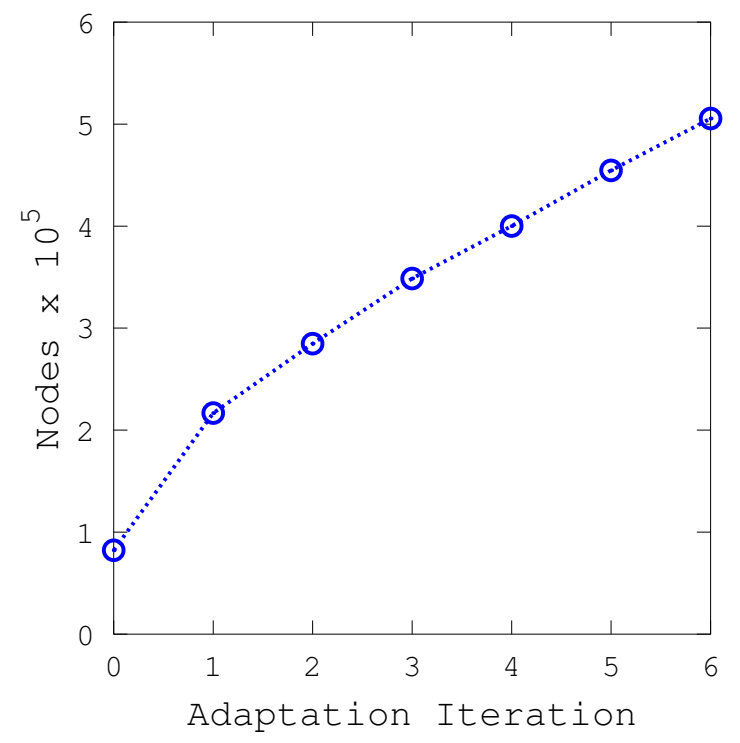

Figure 74: Node increase with adaptation at each iteration 
By observing the change in cell volume from each iteration step, how and where the mesh was adapted is easily distinguished. By calculating the cell volume in each mesh, and dividing them, a ratio of the change in cell volume could be observed such that:

$$
R_{c v}=\frac{V_{n-1}}{V_{n}}
$$

Where $R_{c v}$ is the ratio of change in cell volume, $V$ is the cell volume, and $n$ is the iteration number. If $R_{c v}>1$ the cell volume between the two iterations was reduced (refinement), if $R_{c v}<1$ cell volume was decreased (coarsening), finally if $R_{c v} \approx 1$ there was little to no change in the cell. This gives a straight forward visualization of where and how the mesh was adapted. Results of the change in cell volume are shown in figure 75 for iterations 0 through 6 , and the cumulative change.

Looking at the overall change in $R_{c v}$ in figure 75 the largest change in cell volume occur in the first 4 iterations in the wake of the airfoil, similar to Ref.[84] for a hypersonic case. As the iteration count increases towards 6 , there is less change observed in the domain. Figure $75 \mathrm{f}$ illustrates the overall change in cell volume between iterations 0 and 6 which shows some changes that were not previously observed. Coarsening has appeared around the airfoil itself which did not show in the single consecutive cell ratio plots. This coarsening did however have an affect on the resulting flow field, and will be discussed in the next section.

\section{Adapted Flow Field}

Immediate improvements to the airfoil wake can be seen from the grid adaptation. Figure 76 plots the cycle averaged vorticity magnitude produced by the pitching airfoil using the initial, $2^{\text {nd }}, 4^{\text {th }}$, and $6^{\text {th }}$ adapted meshes. The cycle average was calculated by averaging the vorticity magnitude at each node during two pitching cycles. Unlike the change in cell volume, where the greatest change was observed in the first few iteration, the vorticity continues to become better resolved as the adaptation 


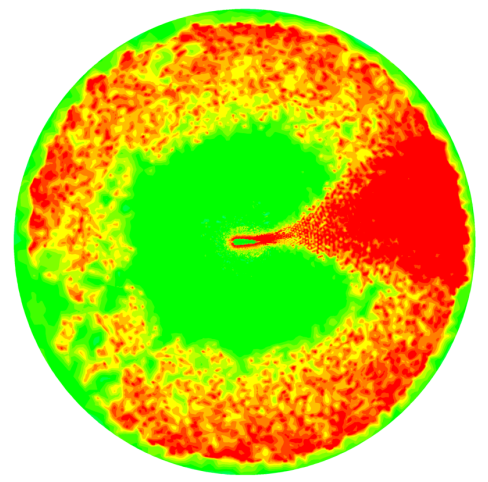

(a) Iteration 0 to 1

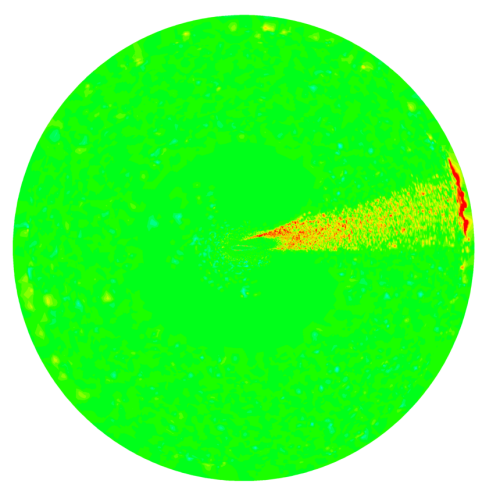

(c) Iteration 2 to 3

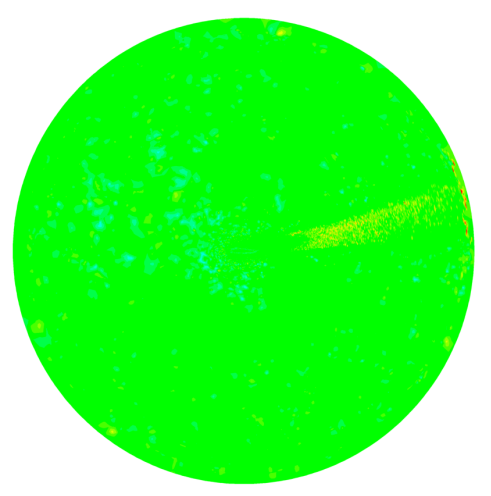

(e) Iteration 4 to 5

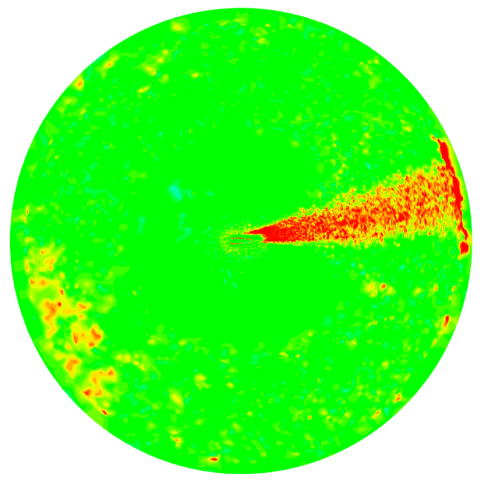

(b) Iteration 1 to 2

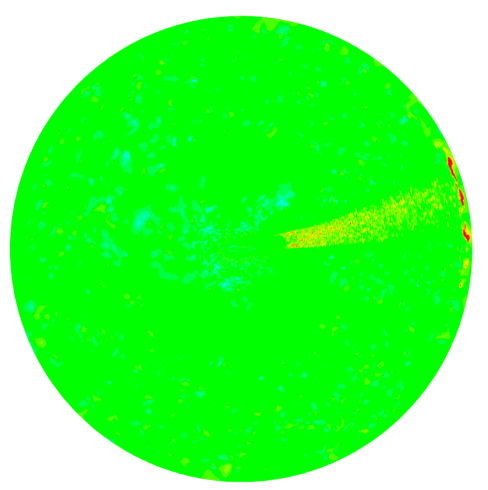

(d) Iteration 3 to 4

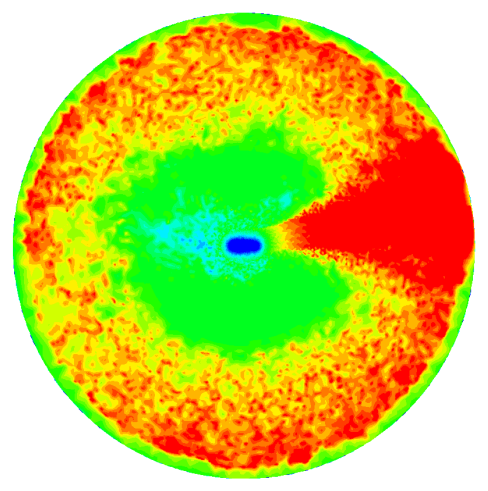

(f) Overall Change (0 to 6)

Figure 75: Change in cell volume ratio $\left(R_{c v}\right)$ between consecutive adaptation iteration steps. (Red) $R_{c v}>1$, (Green) $R_{c v} \approx 1$, (Blue) $R_{c v}<1$ 
continues. After the second adaptation, the wake of the airfoil is carried out twice the distance compared to the initial grid. As the adaptation continues, the average vorticity magnitude increases further downstream, and the wake becomes less diffuse. 


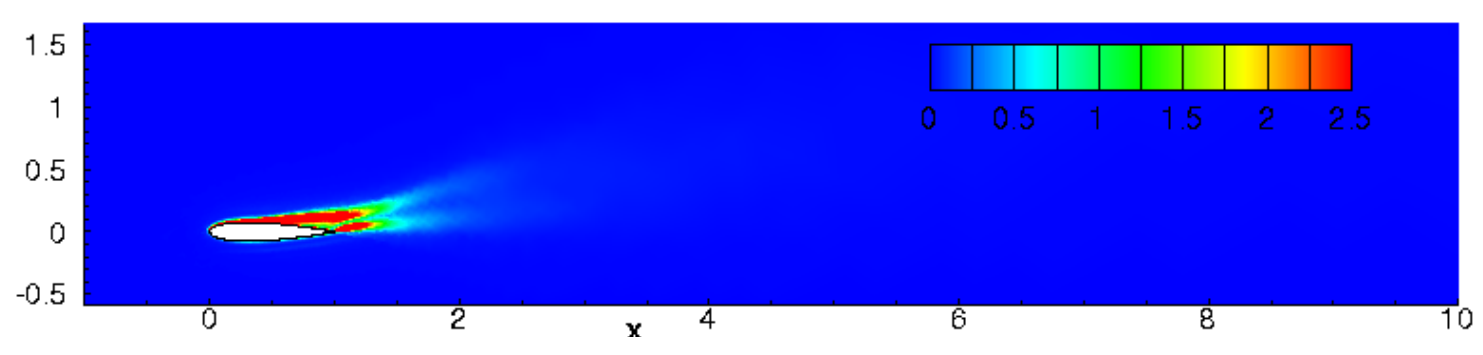

(a) Initial Grid

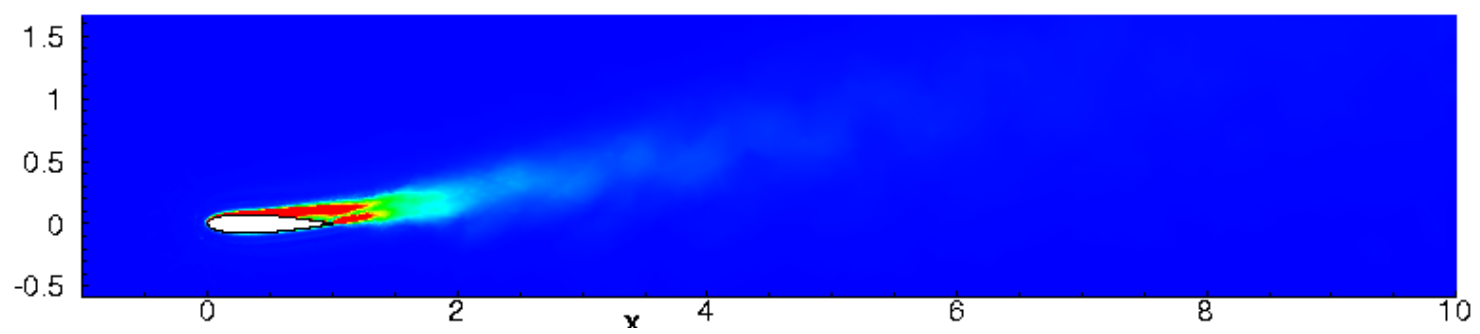

(b) $2^{\text {th }}$ Adaptation

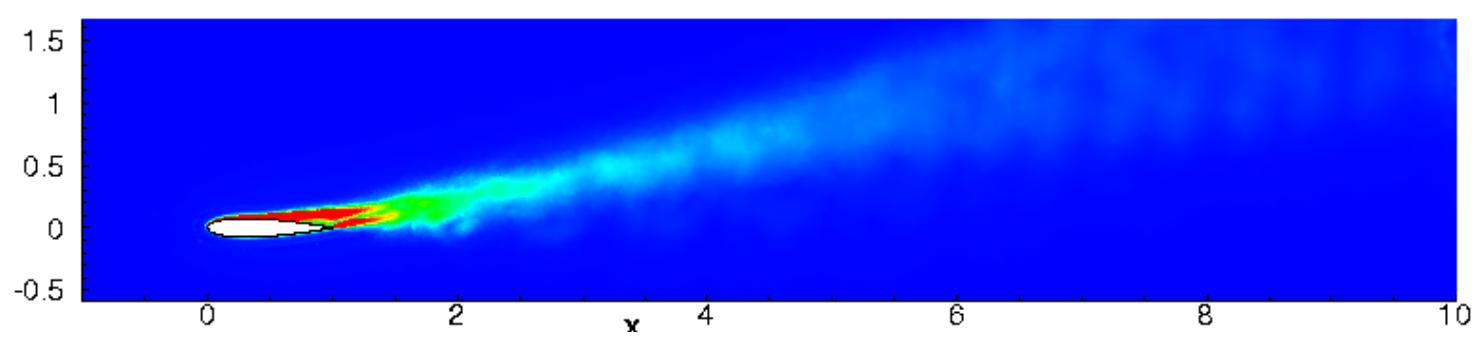

(c) $4^{\text {th }}$ Adaptation

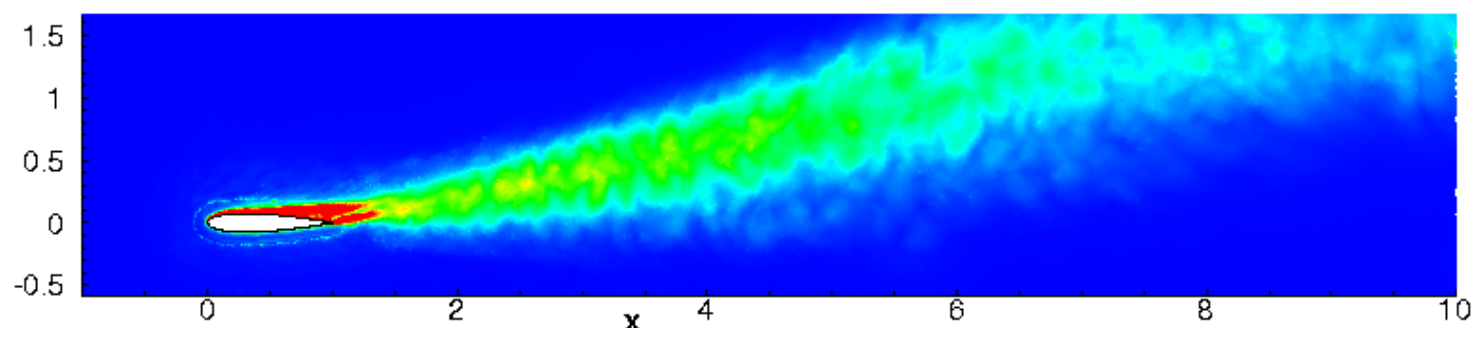

(d) $6^{\text {th }}$ Adaptation

Figure 76: Cycle averaged vorticity magnitude of the initial, $2^{\text {nd }}, 4^{\text {th }}$, and $6^{\text {th }}$ adapted meshes.

As discussed previously, figure $75 \mathrm{f}$ revealed coarsening occurring around the 
airfoil during consecutive adaptation. Snapshots were taken at the mean angle of attack to illustrate the effects of adapted coarsening had on the flow field. The initial mesh (figure 77a) shows vorticity along the upper surface of the airfoil detaching at approximately at $x / c=0.75$. At the second iteration (figure $77 \mathrm{~b}$ ), coarsening of the mesh can be seen near the surface of the airfoil and vorticity detaches sooner (approximately $x / c=0.25$ ). Vorticity begins to be reattached as the adaptation and coarsening continues (figure 77c) but the vorticity magnitude is weaker compared to the initial grid. This is the result of inadequate grid resolution near the surface of the airfoil, which results in the reduction of force coefficients (shown in figure 78).
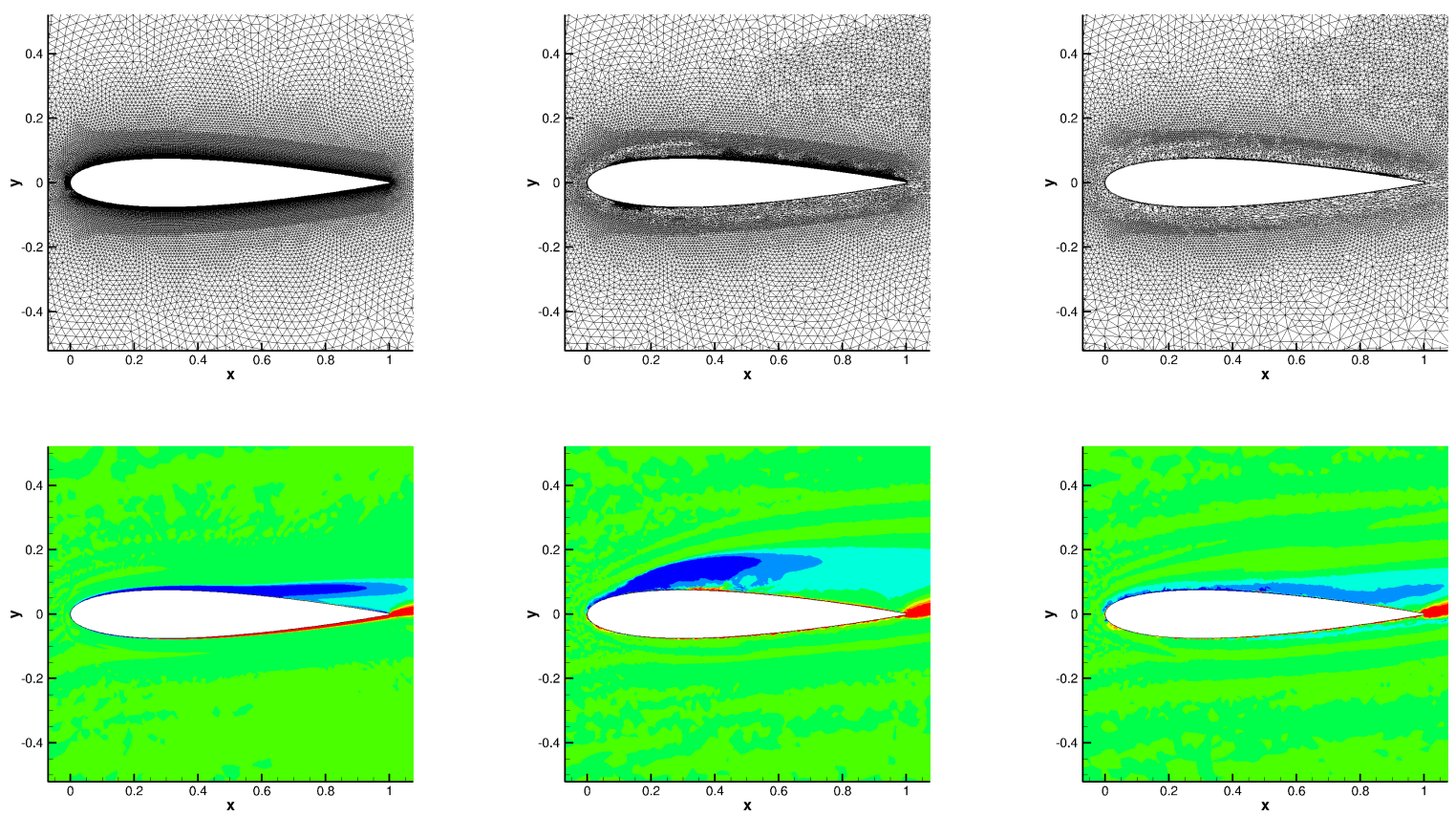

(a) Initial Mesh

(b) Iteration 2

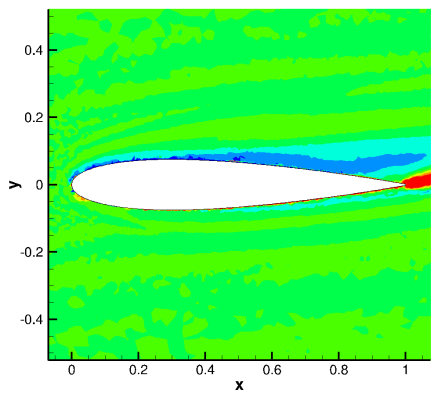

(c) Iteration 6

Figure 77: Coarsening of mesh and changes in vorticity close to the airfoil surface due to mesh adaptation. 


\section{Adapted Forces and Error}

Figures 78 plots the instantaneous lift and drag coefficients for four selected adaptation iterations: the initial, $2^{\text {nd }}, 4^{\text {th }}$, and $6^{\text {th }}$ adapted meshes listed as $I_{0}, I_{2}, I_{4}$, and $I_{6}$ respectively. Like the grid adaptation, the force coefficients show their largest change in the first few adaptations. This change is due to the effect of slow coarsening around the airfoil that occurs throughout the adaptation history observed in figure 75. However, as the grid continues to adapt, the instantaneous forces first diverge away from $I_{0}$ and then begin to converge back towards $I_{0}$.
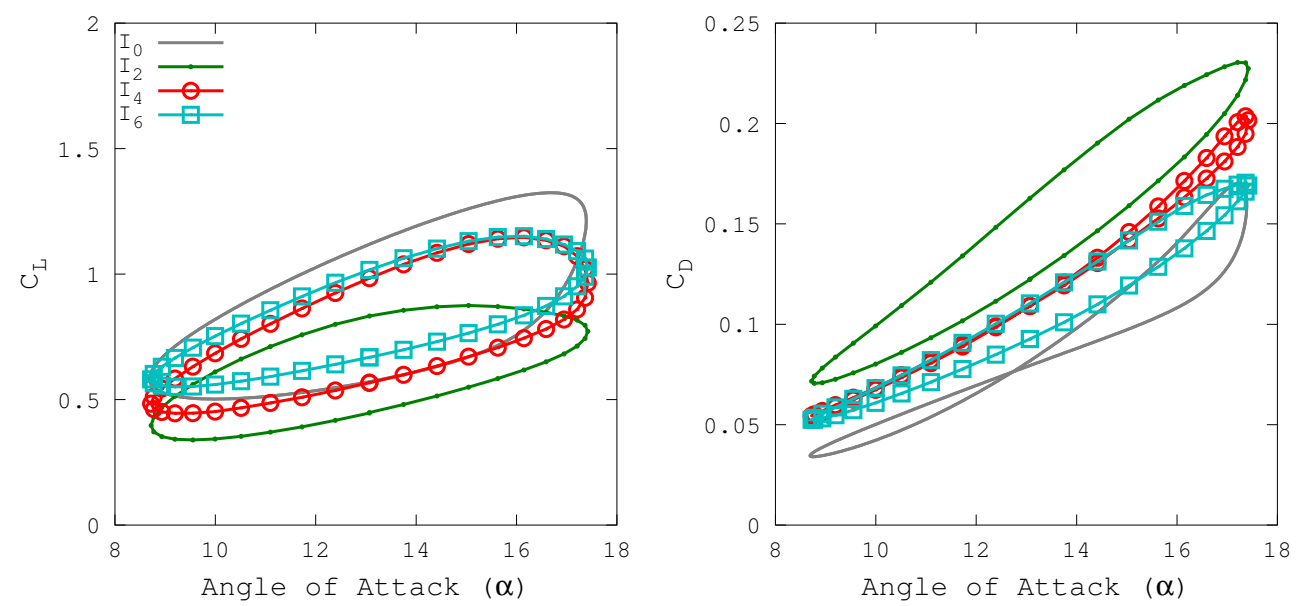

Figure 78: Instantaneous lift, drag, and moment coefficients of the initial, $2^{\text {nd }}, 4^{\text {th }}$, and $6^{\text {th }}$ adapted meshes.

Examining the force averages, and errors (figure 79) show similar results. The two force coefficients were seen to diverge quickly away from the expected trend of approaching the uniform refinement values. The first adaptation results in an approximate $50 \%$ reduction in the lift coefficient, with tripling of the average drag. Due forces changing quickest in the first set of adaptations, the spatial error associated with the adapted grids resulted in a "noisy" error plot. However, adaptations 3 through 6 show a rate of error reduction greater than the uniform refinement. While 
coarsening affected the flow field, the adaptation did continue to reduce the spatial error from the grid.

In summary, the grid adaptation was seen to have its largest effects in the first few iterations. Slow coarsening of the mesh around the airfoil resulted in altered lift and drag coefficients. However, refinement of the wake of the airfoil allowed vorticity to be carried out significantly further than the initial mesh. Thus feature-based adaptation can be an effective means of wake refinement for dynamic stall when the practitioner has no prior knowledge of the wake characteristics. 

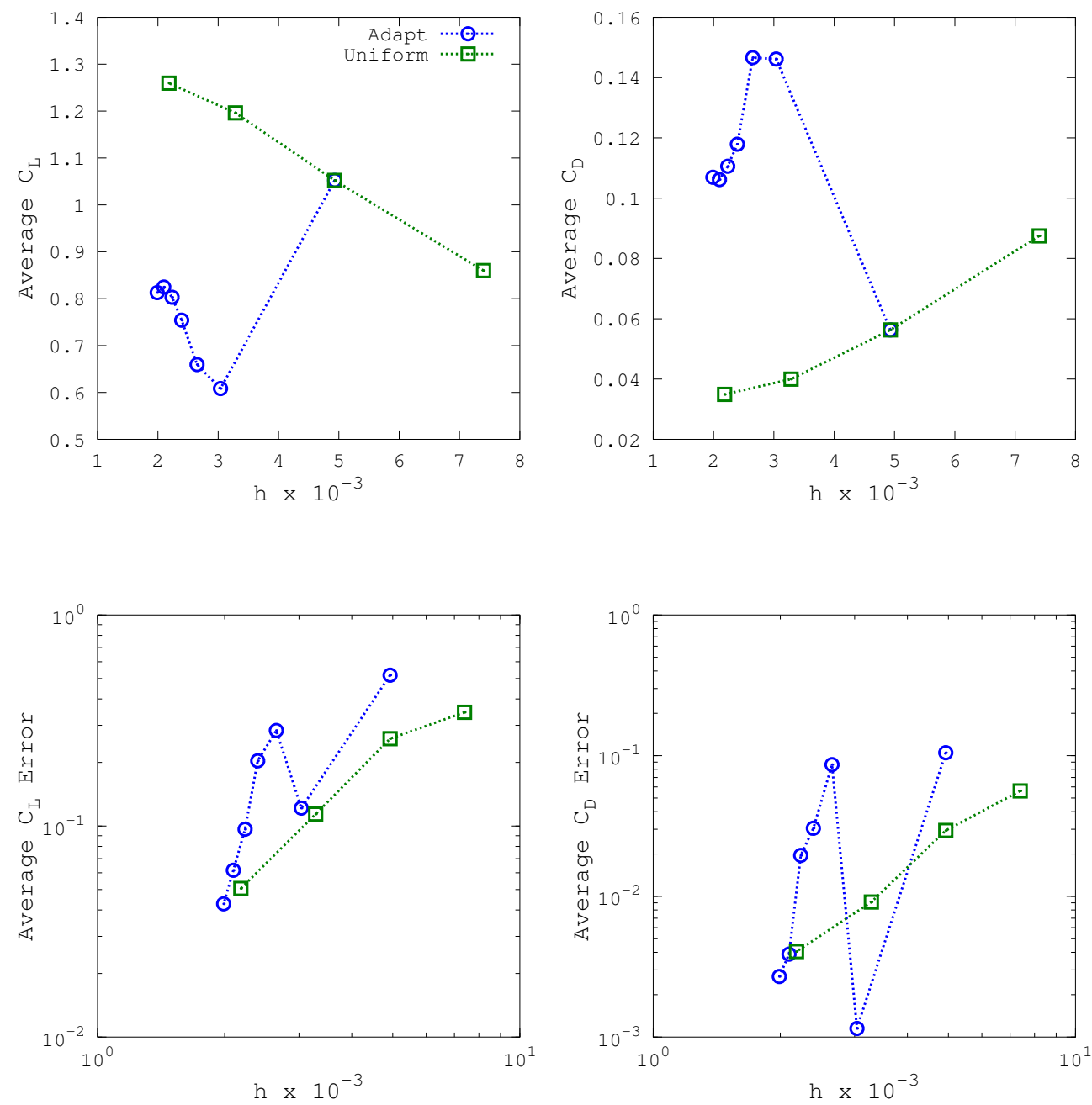

Figure 79: Average and error of lift, drag, and moment coefficients of adapted grids

\section{H. Conclusion}

A dynamic stall motion was selected from a previous experimental study that replicates sinusoidal pitching motion. A family of uniformly refined grids was generated and evaluated to find the reduction in spatial and temporal error associated with each grid. It was found as the grids were uniformly refined, the average spatial error 
for each grid was reduced. The selected time step was found to impact the average forces little, but had a large effect on the instantaneous force profile; primarily the down-stroke of the motion.

For the feature-based adaptation, a single grid and time stepping combination was selected from the uniformly refined grid family, and tested as to whether feature adaption would improve the rate of convergence as the grid resolution was increased. Coarsening occurred around the airfoil altering the vorticity being generated by the wing. This reduced the strength of vorticity on the surface of the airfoil, and initially reduced the instantaneous force coefficients. However, as the adaptation continues, instantaneous forces were seen to converge near their original values. In the examination of the spatial errors, the rate of error reduction was seen to be greater than that of the uniform refinement after the second adaptation. Significant wake refinement was also observed allowing vorticity to be carried well behind the airfoil. 


\section{CHAPTER X}

\section{CONCLUSIONS}

The objective of this dissertation was to numerically investigate the dynamic stall flow phenomena of a flat plate wing with various aspect ratios undergoing a pitchup and perching motion at a Reynolds number of 500. The motions were investigated using 2D and 3D numerical techniques analyzing the simulated aerodynamic forces as well as the circulation developed by the LEV. Special attention was paid the dynamics of the LEV circulation across the span of each wing. The dissertation was broken into several key chapters that examined specific components of the effects of aspect ratio, reduced pitch rate, and motion kinematics.

Reynolds number effects on 2D pitching wings - The pitching motion was found to be Reynolds number sensitive. Peak lift was found to increase with Reynolds number, while the noncirculatory forces added by the rotational acceleration at the beginning of the perching motion were found to be insensitive to Reynolds number. Vortices generated by the motion were also found to be Reynolds number dependent. As Reynolds number increased, the vortices increased in interior vorticity magnitude and became more compact. It was later concluded to be due to the lessening effect of viscosity damping of the the vorticity.

Kinematic effects on pitching and perching wings - Three reduced pitch rates were selected in order to investigate the effect of kinematics on the pitching and perching maneuvers on the resultant lift and drag. As pitch rate increased, lift, drag, and stall angle also increased. The LEV was seen to become smaller with increasing pitch rate with increased vorticity magnitudes. The addition of 
deceleration (perching) resulted in increased lift and drag coefficients, as well as stall angle. The LEV was seen to be smaller in size, and weaker. Additionally the LEV propagated away slowly, and remained attached even at $90^{\circ}$ with the higher pitch rate.

Aspect ratio effects on pitching and perching - Three aspect ratios were tested at the two higher $K$ values. Aspect ratio was seen to increase lift and drag, however it also decreased stall angle. The results were converging to the 2D results with increasing aspect ratio, meaning the flow field and forces were becoming prominently $2 \mathrm{D}$. This was due to the lessening interaction of the tip vortices on the LEV development. At the low aspect ratio, tip vortices weakened the generated LEV. Reduced pitch rate was found to suppress the effects of tip vortices, due to the tip vortices forming slower than the LEV.

Circulatory and noncirculatory analysis of a pitching wings - Forces generated by a pitching wing were seperated into two component forces: circulatory and noncirculatory. Using the Theodorsen model, forces on a 2D wing were separated into lift produced by the LEV (circulatory forces) and apparent mass (noncirculatory forces). Noncirculatory forces were only found to account for 10-20\% of the total forces. The circulation of the LEV was found to match well with the actual lift forces when the LEV was attached to the wing, meaning the LEV is a good indicator of lift produced. This however broke down as the LEV moved away from the wing.

The size of the LEV was found to be independent of the pitching kinematics, and dependent on time. Viscosity diffuses the LEV, resulting in a larger vortex with weaker vorticity.

Circulation development of a 3D LEV - The 2D circulation study was continued with the finite aspect ratio wings; however only the circulation of the LEV was analyzed. Spanwise circulation was seen to increase and become more uni- 
form with aspect ratio. The reduced pitch rate decreased the influence of tip vortices, allowing for LEV circulation to extend to the further along the plate. For the perching wings, the change in pitch rate illustrated the effects of the retention of the LEV after stall. Due to the zero free stream velocity, the LEV stayed attached to the wing extending its influence over a larger angle of attack.

Feature based adaptation of dynamic stall - Work interrelated to the simulation of dynamic stall was also conduced with NASA Langley. Inadequate grid resolution can alter the resulting flow field in the wake of a dynamic stall airfoil. Feature based methods were investigated for their effectiveness in reducing spatial error due to user generated meshes. Feature based adaptation was found to be effective at retaining vortex structures propagating away form the airfoil; however was also found to coarsen mesh in key locations. The adaptation process did recover close to the original forces; however did not improve on overall results.

In summary, the development of the LEV was found to be an important feature in the production of forces by a pitching or perching wing. For MAV development to continue, research must investigate processes to take advantage of the propagation of the LEV. Wing aspect ratio promotes the increases in uniformity of LEV development, while reduced pitch rate determines the timing of the LEV and tip vortex formation.

\section{A. Future Work}

To build upon the work conducted in this dissertation, there are several areas that should be investigated to better our understanding of the LEV development for perching wings.

Vortex Tracking - Understanding how the LEV moves across the wing in both 2D and $3 \mathrm{D}$ can better improve our understanding of the rates at which lift and drag are produced and lost with vortex location. With the addition of vortex 
location, it may be possible to directly calculate the vortex circulation or wing lift from one another.

Improved Vortex Identification - The Q-Criterion is vulnerable to misidentifying vortices in areas of high rotational shear. This commonly occurs in areas where flow is wrapped around a corner (ie: leading edge). Several other methods exist in literature. A recommended scheme is the $\lambda_{2}$ method which uses the velocity orbiting a point to find vortices.

Geometry Effects - Different shaped planforms may reveal different circulation patterns. Specifically insect shaped wings may show improved LEV development and retention, or the lessening effects of tip vortices during perching.

Broader Range of $K$ - In this dissertation, only three reduced rates were tested due to the computational costs involved with the $3 \mathrm{D}$ motions. 


\section{REFERENCES}

[1] R. T. Biedron, V. N. Vatsa, and H. L. Atkins, "Simulation of unsteady flows using an unstructured navier-stokes solver on moving and stationary grids," AIAA paper, vol. 5093, p. 2005, 2005.

[2] J. H. McMasters and M. L. Henderson, "Low-speed single-element airfoil synthesis," 1979.

[3] A. M. Berg and A. A. Biewener, "Wing and body kinematics of takeoff and landing flight in the pigeon (columba livia)," The Journal of experimental biology, vol. 213, no. 10, pp. 1651-1658, 2010.

[4] H.-S. Dou and N. Phan-Thien, "Parallelisation of an unstructured finite volume code with pvm: viscoelastic flow around a cylinder," Journal of non-newtonian fluid mechanics, vol. 77, no. 1, pp. 21-51, 1998.

[5] W. Henshaw, "Ogen: An overlapping grid generator for overture," tech. rep., 1998.

[6] J. Guerrero, Numerical Simulation of the Unsteady Aerodynamics of Flapping Flight. PhD thesis, 2009.

[7] W. Henshaw, "On multigrid for overlapping grid," SIAM Journal on Scientific Computing, vol. 26, pp. 1547-1572, 2005.

[8] K. Granlund, M. Ol, D. Garmann, M. Visbal, and L. Bernal, Experiments and Computations on Abstractions of Perching. Fluid Dynamics and Colocated Conferences, American Institute of Aeronautics and Astronautics, 2010. doi:10.2514/6.2010-4943. 
[9] M. Gad-el Hak, "Micro-air-vehicles: Can they be controlled better?," Journal of aircraft, vol. 38, no. 3, pp. 419-429, 2001.

[10] T. Maxworthy, The Fluid Dynamics of Insect Flight, vol. 13, pp. 329-350. 1981.

[11] M. Platzer, K. Jones, J. Young, and J. Lai, Flapping Wing Aerodynamics: Progess and Challenges, vol. 46, pp. 2136-2149. 2008.

[12] W. Shyy, "Aerodynamics of low reynolds number flyers," Cambridge University Press, 2007.

[13] S. Wang, D. B. Ingham, L. Ma, M. Pourkashanian, and Z. Tao, "Numerical investigations on dynamic stall of low reynolds number flow around oscillating airfoils," Computers and Fluids, vol. 39, no. 9, pp. 1529-1541, 2010.

[14] R. Prater and Y. Lian, "Aerodynamic response of stationary and flapping wings in oscillatory low reynolds number flows," 2012.

[15] P. Saffman and J. Sheffield, "Flow over a wing with an attached free vortex," Studies in Applied Mathematics, vol. 57, pp. 107-117, 1977.

[16] W. J. McCroksey, "The phenomenon of dynamic stall," tech. rep., March 1981.

[17] C. Ford and H. Babinsky, Lift and the Leading Edge Vortex. Aerospace Sciences Meetings, American Institute of Aeronautics and Astronautics, 2012.

[18] J. Ekaterinaris and M. Platzer, "Computational predicitons of airfoil dynamic stall," Progress in Aerospace Science, vol. 33, pp. 759-846, 1997.

[19] L. Carr, "Progress in analysis and production of dynamic stall," Journal of Aircraft, vol. 25, p. 1988, 1988.

[20] C. Shih, L. Lourenco, L. Van Dommenlen, and A. Krothapalli, "Unsteady flow past an airfoil pitching at a constant rate," AIAA Journal, vol. 30, no. 5, pp. 1153-1161, 1992. 
[21] W. J. McCroksey, "Unsteady airfoils," Annual Review of Fluid Mechanics, vol. 14, pp. 285-311, 1982.

[22] M. R. Visbal, "Dynamic stall of a constant-rate pitching airfoil," Journal of Aircraft, vol. 27, no. 5, pp. 400-407, 1989.

[23] M. R. Visbal and J. S. Shang, "Investigation of the flow structure around a rapidly pitching airfoil," AIAA Journal, vol. 27, no. 8, pp. 1044-1051, 1989.

[24] K. Granlund, M. Ol, and L. Bernal, Experiments on Pitching Plates: Force and Flowfield Measurements at Low Reynolds Numbers. American Institute of Aeronautics and Astronautics, 2011. doi:10.2514/6.2011-872.

[25] K. Hord and Y. Lian, Numerical Study of Finite Aspect Ratio Perching Wings. Aerospace Sciences Meetings, American Institute of Aeronautics and Astronautics, 2012.

[26] J. Anderson, "Oscillating foils of high propulsive efficiency," Journal of Fluid Mechanics, vol. 360, pp. 41-72, 1988.

[27] M. Dickinson and K. Gotz, "Unsteady aerodynamic performance of model wings at low reynolds-numbers," Journal of Experimental Biology, vol. 174, pp. 45-64, 1993.

[28] C. Ellington, "Leading-edge vortices in insect flight," Nature, vol. 384, no. 6610, pp. 626-630, 1996.

[29] H. Liu and K. Kawachi, "A numerical study of insect flight," Journal of Computational Physics, vol. 146, no. 1, pp. 142-156, 1998.

[30] G. Kenneth, M. Ol, and L. Bernal, Experiments on Pitching Plates: Force and Flowfield Measurements at Low Reynolds Numbers. Aerospace Sciences Meetings, American Institute of Aeronautics and Astronautics, 2011. 
[31] W. McCroskey, L. Carr, and K. McAlister, "Dynamic stall experiments on oscillating airfoils," AIAA Journal, vol. 14, pp. 57-63, 1976.

[32] H. Liu, T. Nakata, N. Gao, M. Maeda, H. Aono, and W. Shyy, "Micro air vehicle-motivated computational biomechanics in bio-flights: aerodynamics, flight dynamics and maneuvering stability," Acta Mechanica Sinica, vol. 26, no. 6, pp. 863-879, 2010.

[33] R. A. Piziali, C. Ames Research, U. S. A. Aviation, and C. Troop, 2-D and 3-D oscillating wing aerodynamics for a range of angles of attack including stall. Moffett Field, Calif.; Springfield, Va.: National Aeronautics and Space Administration, Ames Research Center : US Army Aviation and Troop Command ; National Technical Information Service, distributor, 1994.

[34] D. M. Tang and E. H. Dowell, "Experimental investigation of three-dimensional dynamic stall model oscillating in pitch," Journal of aircraft., vol. 32, no. 5, p. 1062, 1995.

[35] S. J. Schreck and H. E. Helin, "Unsteady vortex dynamics and surface pressure topologies on a finite pitching wing," Journal of aircraft., vol. 31, no. 4, p. 899, 1994.

[36] F. N. Coton and R. A. M. Galbraith, "An experimental study of dynamic stall on a finite wing," The Aeronautical journal., vol. 103, no. 102, p. 229, 1999.

[37] A. Spentzos, G. Barakos, K. Badcock, B. Richards, P. Wernert, S. Schreck, and M. Raffel, "Investigation of three-dimensional dynamic stall using computational fluid dynamics," AIAA Journal, vol. 43, no. 5, 2005.

[38] T. Yilmaz and D. Rockwell, "Flow structure on finite-span wings due to pitchup motion," Journal of Fluid Mechanics, vol. 691, pp. 518-545, 2012. 
[39] J. Eldredge, C. Wang, and M. Ol, A Computational Study of a Canonical PitchUp, Pitch-Down Wing Maneuver. Fluid Dynamics and Co-located Conferences, American Institute of Aeronautics and Astronautics, 2009.

[40] D. Garmann and M. Visbal, High-Fidelity Simulations of Transitional Flow Over Pitching Airfoils. Fluid Dynamics and Co-located Conferences, American Institute of Aeronautics and Astronautics, 2009.

[41] M. OL, A. Altman, J. Jeff, Eldredge, D. Garmann, and Y. Lian, Rsum of the AIAA FDTC Low Reynolds Number Discussion Group's Canonical Cases. Aerospace Sciences Meetings, American Institute of Aeronautics and Astronautics, 2010.

[42] G. Torres and T. Mueller, Aerodynamic Characteristics of Low Aspect Ratio Wings at Low Reynolds Numbers, pp. 115-141. Progress in Astronautics and Aeronautics, American Institute of Aeronautics and Astronautics, 2001.

[43] M. Okamoto and A. Azuma, "Aerodynamic characteristics at low reynolds numbers for wings of various planforms," AIAA Journal, vol. 49, no. 6, pp. 11351150, 2011.

[44] M. Mizoguchi and H. Itoh, Effect of Aspect Ratio on Aerodynamic Characteristics of Rectangular Wings at Low Reynolds Numbers. Aerospace Sciences Meetings, American Institute of Aeronautics and Astronautics, 2012.

[45] J. Eldredge and C. Wang, Improved low-order modeling of a pitching and perching plate. Fluid Dynamics and Co-located Conferences, American Institute of Aeronautics and Astronautics, 2011.

[46] J. Tannehill, D. Anderson, and R. Pletcher, Computational Fluid Mechanics And Heat Transfer. Taylor \& Francis, 1997. 
[47] W. Henshaw, "A fourth-order accurate method for the incompressible navierstokes equations on overlapping grids," Journal of Computational Physics, vol. 113, no. 1, pp. 13-25, 1994.

[48] W. Henshaw, H. Kreiss, and L. Reyna, "A fourth-order accurate method for the incompressible navier-stokes equations," Journal of Computers and Fluids, vol. 23, pp. 575-593, 1994.

[49] J. Hunt, A. Wray, and P. Moin, "Eddies, stream, and convergence zones in turbulent flows," tech. rep., 1988.

[50] J. F. Thompson, B. K. Soni, and N. P. Weatherhill, Handbook of Grid Generation. CRC Press, 1998.

[51] M. Aftosmis, M. Berger, J. Melton, M. Aftosmis, M. Berger, and J. Melton, Robust and efficient Cartesian mesh generation for component-based geometry. Aerospace Sciences Meetings, American Institute of Aeronautics and Astronautics, 1997. doi:10.2514/6.1997-196.

[52] W. D. Henshaw and N. A. Petersson, "A split-step scheme for the incompressible navier-stokes equations," Numerical simulations of incompressible flows (Half Moon Bay, CA, 2001), pp. 108-125, 2003.

[53] E. A. Volkov, "A finite difference method for finite and infinite regions with piecewise smooth boundary," Dokl. Akad. Nauk SSR, vol. 168, no. 5, pp. 978981, 1968.

[54] G. Starius, "Composite mesh difference methods for elliptic boundary value problems," Numerische Mathematik, vol. 28, no. 2, pp. 243-258, 1977.

[55] J. L. Steger, F. C. Dougherty, and J. A. Benek, A Chimera Grid Scheme, vol. ASME FED-Vol 5. 1983. 
[56] J. Benek, P. Buning, and J. Steger, A 3-D chimera grid embedding technique. Meeting Paper Archive, American Institute of Aeronautics and Astronautics, 1985. doi:10.2514/6.1985-1523.

[57] R. Meakin and N. Suhs, Unsteady aerodynamic simulation of multiple bodies in relative motion. Fluid Dynamics and Co-located Conferences, American Institute of Aeronautics and Astronautics, 1989. doi:10.2514/6.1989-1996.

[58] G. Chesshire and W. Henshaw, "Composite overlapping meshes for the solution of partial differential equations.," Journal of Computational Physics, no. 90, pp. 1-64, 1990.

[59] R. Noack and R. Suggar, "A general capability for moving body overset cfd," in Proceedings of 7th Symposium on Overset Composite Grid and Solution Technology.

[60] D. Calhoun, "A cartesian grid method for solving the two-dimensional streamfunction-vorticity equations in irregular regions," Journal of Computational Physics, vol. 176, no. 2, pp. 231-275, 2002.

[61] J.-I. Choi, R. C. Oberoi, J. R. Edwards, and J. A. Rosati, "An immersed boundary method for complex incompressible flows," Journal of Computational Physics, vol. 224, no. 2, pp. 757-784, 2007.

[62] J. Kim and H. Choi, "Distributed forcing of a flow over a circular cylinder," Physics of Fluids, vol. 17, no. 3, 2005.

[63] O. Posdziech and R. Grundmann, "A systematic approach to the numerical calculation of fundamental quantities of the two-dimensional flow over a circular cylinder," Journal of Fluids and Structures, vol. 23, no. 3, pp. 479-499, 2007.

[64] D. Russell and Z. Wang, "A cartesian grid method for modeling multiple moving 
objects in 2d incompressible viscous flow," J. Comput. Phys., vol. 191, no. 1, pp. $177-205,2003$.

[65] D. J. Tritton, "Experiments on the flow past a circular cylinder at low reynolds numbers," Journal of Fluid Mechanics, vol. 6, no. 04, pp. 547-567, 1959.

[66] M. R. Visbal, "Numerical investigation of deep dynamic stall of a plunging airfoil," AIAA journal, vol. 49, no. 10, pp. 2152-2170, 2011.

[67] M. R. Visbal and D. V. Gaitonde, "High-order-accurate methods for complex unsteady subsonic flows," AIAA journal, vol. 37, no. 10, pp. 1231-1239, 1999.

[68] K. Hord and Y. Lian, Numerical Investigation of the Aerodynamic and Structural Characteristics of a Corrugated Airfoil. Fluid Dynamics and Colocated Conferences, American Institute of Aeronautics and Astronautics, 2010. doi:10.2514/6.2010-4624.

[69] P. Cosyn and J. Vierendeels, Numerical Investigation of Low Aspect Ratio Wings at Low Reynolds Numbers. Fluid Dynamics and Co-located Conferences, American Institute of Aeronautics and Astronautics, 2005.

[70] G. E. Torres, Aerodynamics of low aspect ratio wings at low Reynolds numbers with applications to micro air vehicle design. 2002.

[71] W. Shyy, P. Trizila, C.-k. Kang, and H. Aono, "Can tip vortices enhance lift of a flapping wing?," AIAA journal, vol. 47, no. 2, pp. 289-293, 2009.

[72] K. D. Jones and M. Platzer, "On the prediction of dynamic stall onset on airfoils in low speed flow," in 8th International Symposium on Unsteady Aerodynamics and Aeroelasticity of Turbomachines.

[73] J. G. Leishman and T. S. Beddoes, "A generalized model for airfoil unsteady aerodynamic behavior and dynamic stall using the indical method," in Proceedings of the 42nd Annual forum of the American Helicopter Society, 1986. 
[74] A. Jones and H. Babinsky, Three-Dimensional Effects on a Waving Wing. Aerospace Sciences Meetings, American Institute of Aeronautics and Astronautics, 2010.

[75] S. Venkata and A. Jones, Leading-Edge Vortex Structure over Multiple Revolutions of a Rotating Wing, vol. 50, pp. 1312-1316. 2013.

[76] Y. Baik, L. Bernal, K. Granlund, and M. Ol, Unsteady Force Generation and Vortex Dynamics of Pitching and Plunging Aerofoils, vol. 709, pp. 37-68. 2012.

[77] K. Hord and Y. Lian, "Leading edge vortex development on a pitch-up airfoil," in ASME 2013 Fluids Engineering Division Summer Meeting, American Society of Mechanical Engineers, 2013.

[78] C. Wojcik and J. Buchholz, Parameter Variation and the Leading-Edge Vortex of a Rotating Flat Plate, vol. 52. 2014.

[79] T. Theodorsen, "General theory of aerodynamic instability and the mechanism of flutter," vol. NACA Rept. 496, 1935.

[80] R. Kiran, G. Ashok, E. Jack, O. Michael, and G. Kenneth, Theoretical, Computational and Experimental Studies of a Flat Plate Undergoing High-Amplitude Pitching Motion. Aerospace Sciences Meetings, American Institute of Aeronautics and Astronautics, 2011.

[81] K. Granlund, M. Ol, and L. Bernal, Unsteady Pitching Flate Plates, vol. 733. 2013.

[82] A. G. Kravchenko and P. Moin, "Numerical studies of flow over a circular cylinder at re=3900," Physics of fluids, vol. 12, p. 403, 2000.

[83] N. M. Komerath, M. J. Smith, and C. Tung, "A review of rotor wake physics and modeling," Journal of the American Helicopter Society, vol. 56, no. 2, pp. 22006-1-22006-19, 2011. 
[84] K. L. Bibb, P. A. Gnoffo, M. A. Park, and W. T. Jones, "Parallel, gradientbased anisotropic mesh adaptation for re-entry vehicle configurations," in 9th AIAA/ASME Joint Thermophysics and Heat Transfer Conference, 2006.

[85] H. Kang and O. Kwon, "Effect of wake adaptation on rotor hovering simulations using unstructured meshes," Journal of Aircraft, vol. 38, no. 5, 2001.

[86] Y. Park and O. Kwon, "Simulation of unsteady rotor-fuselage interactions using unstructured adaptive sliding meshes," Journal of Aircraft, vol. 49, no. 4, 2006.

[87] G. W. W. A. J. Thomas and S. Krist, "Grid convergence for adaptive methods," AIAA, vol. 10th Computational Fluid Dynamics Conference, no. 91-1592, 1991.

[88] P. Sweby and H. Yee, "On the dynamics of some grid adaptation sciences," in Proc. 4th Int. Conf on Numerical Grid Generation in Computational Fluid Dynamics and Related Fields, Swansea, UK, 1994.

[89] T. J. Baker, "Mesh adaptation strategies for problems in fluid dynamics," Finite Elements in Analysis and Design, vol. 25, no. 34, pp. 243 - 273, 1997. ¡ce:title¿Adaptive Meshing, Part 2i/ce:title

[90] W. K. Anderson and D. L. Bonhaus, "An implicit upwind algorithm for computing turbulent flows on unstructured grids," Computers \& Fluids, vol. 23, no. 1, pp. 1-21, 1994.

[91] V. N. Vatsa and M. H. Carpenter, "Higher order temporal schemes with error controllers for unsteady navier-stokes equations," AIAA Paper, vol. 5245, p. 2005, 2005.

[92] C. L. Rumsey, M. D. Sanetrik, R. T. Biedron, N. D. Melson, and E. B. Parlette, "Efficiency and accuracy of time-accurate turbulent navier-stokes computations," Computers \& Fluids, vol. 25, no. 2, pp. 217-236, 1996. 
[93] J. Peraire, M. Vahdati, K. Morgan, and O. C. Zienkiewicz, "Adaptive remeshing for compressible flow computations," Journal of computational physics, vol. 72, no. 2, pp. 449-466, 1987.

[94] M. A. Park, "Three-dimensional turbulent rans adjoint-based error correction," AIAA paper, vol. 3849, pp. 1-14, 2003.

[95] M. A. Park and D. L. Darmofal, "Parallel anisotropic tetrahedral adaptation," AIAA Paper, vol. 917, p. 2008, 2008.

[96] M. A. Park, Anisotropic output-based adaptation with tetrahedral cut cells for compressible flows. PhD thesis, Massachusetts Institute of Technology, 2008.

[97] M. A. Park, "Adjoint-based, three-dimensional error prediction and grid adaptation," AIAA journal, vol. 42, no. 9, pp. 1854-1862, 2004.

[98] E. Lee-Rausch, M. Park, W. Jones, D. Hammond, and E. Nielsen, "Application of parallel adjoint-based error estimation and anisotropic grid adaptation for three-dimensional aerospace configurations," AIAA paper, vol. 4842, p. 2005, 2005.

[99] L. F. Richardson and J. A. Gaunt, "The deferred approach to the limit. part i. single lattice. part ii. interpenetrating lattices," Philosophical Transactions of the Royal Society of London. Series A, containing papers of a mathematical or physical character, vol. 226, pp. 299-361, 1927.

[100] M. State, "Advancing-front/local-reconnection," January 2013. Version 12.28.57. 


\section{APPENDIX}

\section{Nomenclature}

$\begin{array}{llll}\text { AR } & \text { Aspect ratio } & \mathbf{C W} & \text { Clockwise } \\ \mathbf{a} & \text { Smoothing parameter } & \mathbf{\Gamma} & \text { Circulation } \\ \mathbf{A} & \text { Area } & \mathbf{K} & \text { Reduced pitching rate } \\ \alpha & \text { Angle of attack } & \mathbf{L E V} & \text { Leading edge vortex } \\ \dot{\alpha} & \text { Rotational velocity } & \omega_{\mathbf{z}} & \text { Vorticity about the z-axis } \\ \ddot{\alpha} & \text { Rotational acceleration } & \mathbf{Q} & \text { Q-Criterion } \\ \mathbf{b} & \text { Span length } & \mathbf{r} & \text { Radius } \\ \mathbf{c} & \text { Chord } & \mathbf{R e} & \text { Reynolds number } \\ \mathbf{C}_{\mathbf{D}} & \text { Drag coefficient } & \mathbf{t} & \text { Time } \\ \mathbf{C}_{\mathbf{L}} & \text { Lift coefficient } & \mathbf{U} & \text { Free stream velocity } \\ \mathbf{C}_{\mathbf{L}, \mathbf{C}} & \text { Circulatory lift coefficient } & \mathbf{V} & \text { Wing velocity } \\ \mathbf{C}_{\mathbf{L}, \mathbf{M}} & \text { A lift coefficient } & \dot{\mathbf{V}} & \text { Wing deceleration } \\ \mathbf{C}_{\mathbf{L}, \mathbf{N C}} & \text { Noncirculatory lift coefficient } & \mathbf{z} & \text { Span location } \\ \mathbf{C}_{\mathbf{N}} & \text { Normal force } & & \end{array}$




\section{CURRICULUM VITAE}

Kyle M. Hord

Phone: (615)-438-7900 or Email: kmhord01@gmail.com

EDUCATION $\quad \mathrm{PhD}$ in Mechanical Engineering

August 2014

J.B. Speed School of Engineering

University of Louisville, Louisville, KY

Dissertation: Numerical Study of Leading Edge Vortex Circulation

Development on Finite Aspect Ratio Perching Wing

M.Eng in Mechanical Engineering

August 2010

J.B. Speed School of Engineering

University of Louisville, Louisville, KY

Thesis: Numerical Investigation of the Aerodynamic and Structural

Characteristics of a Corrugated Airfoil

B.S. in Mechanical Engineering

J.B. Speed School of Engineering

University of Louisville, Louisville, KY

December 2009

JOURNAL Lian, Y., Broering, T., Hord, K., and Prater, R., "The Characterization of Tandem

PUBLICATIONS and Corrugated Wings, " Progress of Aerospace Sciences, Vol. 65, February 2014, pp 41-69

Kyle Hord and Yongsheng Lian, "Numerical Investigation of the Aerodynamic and Structural Characteristics of a Corrugated Airfoil," Journal of Aircraft, Vol. 49, No. 4, May-June 2012, pp 749-757

CONFERENCE
PUBLICATIONS

Kyle Hord and Yongsheng Lian, "Feature-Based Grid Adaption for the Study of DyPUBLICATIONS namic Stall, AIAA-2014-2997, 32nd AIAA Applied Aerodynamics Conference, Atlanta, GA, June 16-20, 2014

Kyle Hord and Yongsheng Lian,"Leading Edge Vortex Dynamics on a Pitch-up Airfoil, Proceedings of the ASME 2013 Fluid Engineering Division Summer Meeting, June 2013, FEDSM2013-16019

Yan Chen, Kyle Hord, Russell Prater, Yongsheng Lian and Lihui Bai, "Design Optimization of a Vertical Axis Wind Turbine Using a Genetic Algorithm and Response Surface Methodology", 14th AIAA/ISSMO Multidisciplinary Analysis and Optimization Conference, Indianapolis, Indiana, September 1719, 2012

Kyle Hord and Yongsheng Lian, "Numerical Study of a Low Aspect Ratio Wing Undergoing a Perching Maneuver," AIAA-2012, 50th AIAA Aerospace Sciences Meeting, Nashville, TN, January 9 -12, 2012

Kyle Hord and Yongsheng Lian, "Numerical Investigation of the Aerodynamic and Structural Characteristics of a Corrugated Airfoil," AIAA-2010-4624, 40th Fluid Dynamics Conference and Exhibit, Chicago, Illinois, June 28 July 1, 2010 
University of Louisville, Louisville, KY

- Class size of 40 students, developed own teaching curriculum.

- Covered thermodynamic analysis of common heat engines: Rankine, Brayton, Otto, diesel, and heat pumps.

- Rated $4.04 / 5$ by students with a department average of 3.96 at the end of the semester.

\section{EMPLOYMENT Ph.D Candidate}

Computational Thermo Fluids Laboratory

May 2009 - August 2014

University of Louisville, Louisville, KY

- Performed computational analysis on corrugated wings at ultra-low Reynolds number

- Investigated leading edge vortex development dynamics on pitching airfoils

- Maintained six 32-core Linux servers used by laboratory and trained new students in Linux software.

NASA Langley Aerospace Research Scholar

June-August 2014

Computational Aerosciences Branch

NASA Langley Research Center, Hampton, VA

- Implemented unsteady goal oriented mesh adaptation in FUN3D

- Reduces spatial error assosiated with computational meshes

- Verified Euler and laminar implementations

NASA Langley Aerospace Research Scholar

June-August 2013

Computational Aerosciences Branch

NASA Langley Research Center, Hampton, VA

- Investigated spatial and temporal convergence on feature based adapting grids on deep dynamic stall wings.

Mechanical Co-op Engineer

January 2007-August 2008

Project Engineering

E.ON US - LG\&E, Louisville, KY

- Developed preliminary design of a fuel delivery system to be used in start-up of a power plant boiler.

- Coordinated hydro-excavation of cooling tower pipe for servicing and inspection.

- Coordinated removal and relocation of limestone processing equipment.

\section{APPLIED

Wilhelm Solar Flight Competition

January 2014 - May 2014

- Designed and built a solar powered airship with thrust vectoring.

- Placed $1^{\text {st }}$ out of 6 teams.

NASA University Student Launch Initiative

July 2012 - May 2013

Co-Captain and Recovery Captain

- Assisted in managing team affairs: University and public relations, finances, and technical documentation.

- Developed a continuous dis-reefing parachute system for optimal descent velocity control.

- Placed $2^{\text {nd }}$ out of 39 teams, awarded "Best Vehicle Design, Educational Outreach, and Best Website." 
Department Chair Selection Committee

March 2013 - July 2013

Student Representative

- Reviewed, and interviewed candidates for the position of Mechanical Engineering Department Chair.

- Represented student's interest in evaluating candidates.

Wilhelm Solar Flight Competition

August 2012 - May 2013

Captain and Airframe Design Lead

- Designed and built a solar power aircraft out of inexpensive materials and supplies.

- Trained team members in safety, and operation of remote control aircraft.

- Placed $1^{\text {st }}$ out of 8 teams.

NASA University Student Launch Initiative

July 2011 - May 2012

Co-Captain and Recovery Captain

- Designed and built an autonomous recovery system for a high powered rocket.

- Coordinated with computer science students to design a feasible guidance system.

- Placed $5^{\text {th }}$ out of 42 teams, awarded "Best Rookie Team of the Year."

Cardinal Computational Fluid Dynamics

April 2010 - August 2012

President

- Founded to help find students interested in fluid dynamics research.

- Arranged and organized student trips to conferences.

AWARDS

Speed School National Scholars

August 2005

NASA Kentucky Graduate Fellowship

November 2010

- Awarded to students addressing the challenges of aerospace research related to NASA's strategic goals 